\title{
The Crystal Collimation System of the Relativistic Heavy Ion Collider
}

Raymond Patrick Fliller III

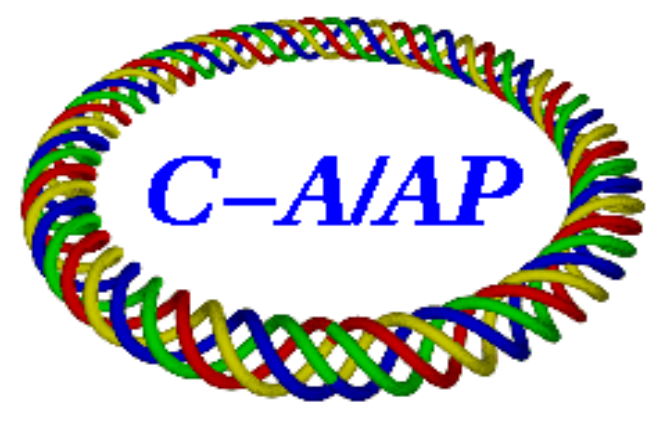

Collider-Accelerator Department Brookhaven National Laboratory Upton, NY 11973 


\section{The Crystal Collimation System of the Relativistic Heavy Ion Collider}

A Dissertation Presented

by

Raymond Patrick Fliller III

to

The Graduate School

in Partial fulfillment of the

Requirements

for the Degree of

Doctor of Philosophy

in

Physics

Stony Brook University

December 2004 
Copyright by

Raymond Patrick Fliller III

2004 


\section{Stony Brook University}

The Graduate School

$\underline{\text { Raymond Patrick Fliller III }}$

We, the dissertation committee for the above candidate for the

Doctor of Philosophy degree,

hereby recommend acceptance of this dissertation.

\section{Dr. Stephen G. Peggs, Adjunct Professor, Dept. of Physics and Astronomy Dissertation Adviser}

Dr. Thomas K. Hemmick, Professor, Dept. of Physics and Astronomy Chairperson of Defense

\footnotetext{
Dr. John Smith, Professor, Dept. of Physics and Astronomy

Dr. K. Angelika Drees, Physicist, Collider-Accelerator Dept. Brookhaven National Laboratory
}

This dissertation is accepted by the Graduate School

Dean of the Graduate School 


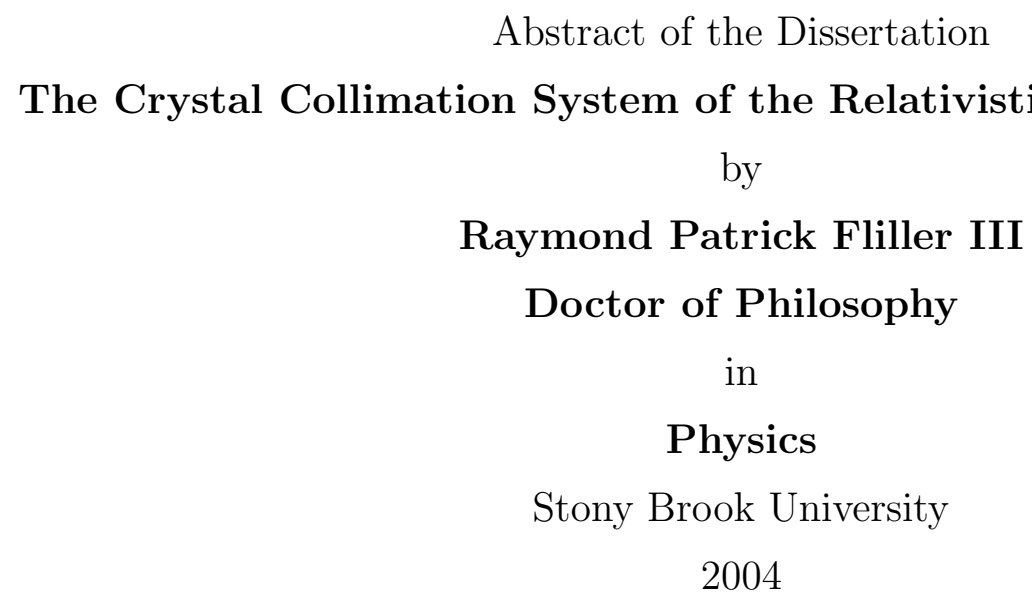

Crystal Channeling occurs when an ion enters a crystal with a small angle with respect to the crystal planes. The electrostatic interaction between the incoming ion and the lattice causes the ion to follow the crystal planes. By mechanically bending a crystal, it is possible to use a crystal to deflect ions. One novel use of a bent crystal is to use it to channel beam halo particles into a downstream collimator. By deflecting the halo particles into a collimator with a crystal it may be possible to improve the collimation efficiency as compared to a conventional two stage collimation system.

A bent crystal was installed in the counterclockwise ring of the Relativistic Heavy Ion Collider (RHIC) prior to the FY2001 run to be used as the first stage of a two stage collimation system. We present a model and simulations to the predict crystal channeling efficiency. The simulations and model predict a channeling efficiency between $59 \%$ and $74 \%$ depending on the choice of optics.

Attempts to reduce backgrounds in RHIC experiments using the crystal were unsuccessful because of the low channeling efficiency. Channeling results show a channeling efficiency $75 \%$ lower than originally predicted. Errors in the horizontal Twiss parameters at the crystal and inaccurate assumptions of the beam halo distribution are the cause of the large discrepancy. The strong scattering from the crystal made it impossible to intercept most of the scattered ions from the crystal using a single 
downstream absorber. Background at the STAR detector downstream of the crystal collimator was increased by as much as a factor of two and was rarely reduced below the uncollimated background rate. As a result, the crystal collimator was removed and the collimation system upgraded to a standard two stage system for the FY2004 gold - gold run.

We also discuss the uses and effectiveness of the copper scrapers used during all RHIC runs. Measurements of diffusion rates and frequency analysis of beam losses were performed. These measurements aid in the understanding of beam growth rates. We performed beam profile measurements using the scrapers.

The design and use of the upgraded collimation system is discussed. Future plans for collimation at RHIC are examined. 
And God hath given to me to speak as I would, and to conceive thoughts worthy of those things that are given me: because he is the guide of wisdom, and the director of the wise: For in his hand are both we, and our words, and all wisdom, and the knowledge and skill of works. For he hath given me the true knowledge of the things that are: to know the disposition of the whole world, and the virtues of the elements, The beginning, and ending, and midst of the times, the alterations of their courses, and the changes of seasons, The revolutions of the year, and the dispositions of the stars, The natures of living creatures, and rage of wild beasts, the force of winds, and reasonings of men, the diversities of plants, and the virtues of roots, And all such things as are hid and not foreseen, I have learned: for wisdom, which is the worker of all things, taught me.

The Book of Wisdom 7:15-21

Saint Albert the Great, Patron of the Natural Sciences, pray for us. 


\section{Contents}

List of Figures xiii

$\begin{array}{ll}\text { List of Tables } & \text { XV }\end{array}$

Acknowledgments $\quad$ xvi

1 Introduction 1

1.1 Relativistic Heavy Ion Collider . . . . . . . . . . . . . . 2

1.2 Linear Beam Dynamics . . . . . . . . . . . . . 5

1.2.1 Equations of Motion ............... 5

1.2.2 Solution of the Equations of Motion . . . . . . . . . . . . 7

1.2 .3 Dispersion ........................ 11

1.2.4 Constants of the Motion ............... 13

1.3 Need for collimation . . . . . . . . . . . . . . 15

2 Crystal Collimation $\quad 21$

2.1 Crystal Channeling . . . . . . . . . . . . . . . . . 21

2.1.1 Crystal Structure . . . . . . . . . . . . . . 22

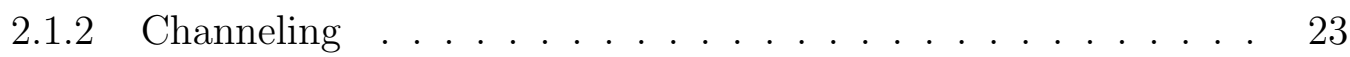

2.1.3 Crystal Acceptance . . . . . . . . . . . . . . . 28

2.1.4 Energy Loss . . . . . . . . . . . . . . . . 31 
2.1.5 Dechanneling . . . . . . . . . . . . . . . . . . . 33

2.1.6 Volume Capture . . . . . . . . . . . . . . . . . . . . . . . 34

2.2 Collimation . . . . . . . . . . . . . . . . 36

2.2.1 Two Stage Collimation . . . . . . . . . . . . . . . . . . 36

2.2.2 Two Stage Crystal Collimation . . . . . . . . . . . . . . 43

3 The RHIC Collimation System $\quad 51$

3.1 Hardware . . . . . . . . . . . . . . . . . . . . . 52

3.1 .1 Crystal . . . . . . . . . . . . . . . . . . . 53

$3.1 .2 \quad$ Crystal Vessel . . . . . . . . . . . . . . . . . . . . . 54

$3.1 .3 \quad$ Laser System . . . . . . . . . . . . . . . . . . 55

3.1 .4 Copper Scraper . . . . . . . . . . . . . . . . . . . . . 60

3.1 .5 Detectors . . . . . . . . . . . . . . . 61

3.2 Beam Parameters . . . . . . . . . . . . . . . . . . . 64

3.3 Controls Software . . . . . . . . . . . . . . . . 65

3.3 .1 PET Page . . . . . . . . . . . . . . . . . 66

3.3 .2 Crystal Control Program . . . . . . . . . . . . . . 66

3.3.3 Scraper Control Program . . . . . . . . . . . . . . . . . 68

3.4 Data Logging . . . . . . . . . . . . . . . . . . . . . . . . 69

3.5 Data Analysis Program . . . . . . . . . . . . . . . . . . . 69

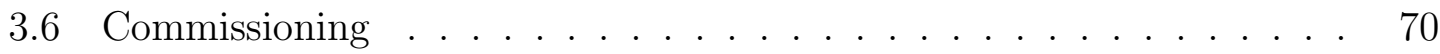

4 Simulations $\quad 72$

4.1 Software . . . . . . . . . . . . . . . . . 73

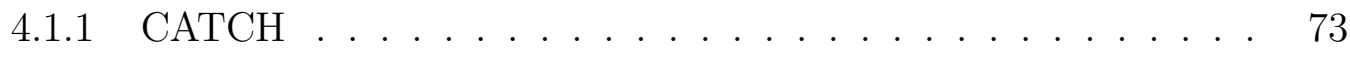

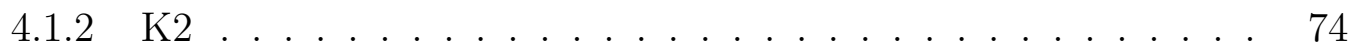

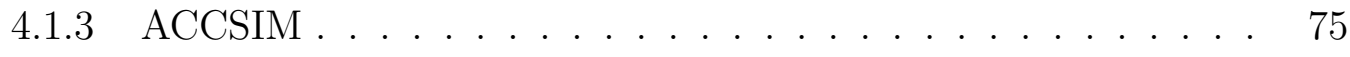




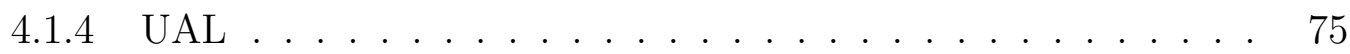

4.1 .5 scan $\ldots \ldots \ldots \ldots \ldots \ldots \ldots \ldots \ldots \ldots$

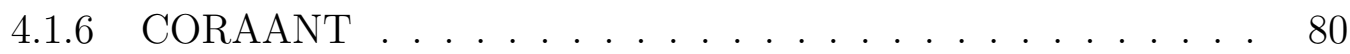

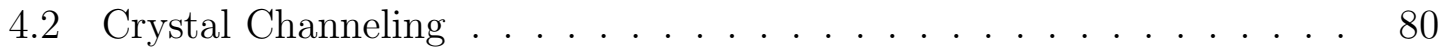

4.3 Collimation Efficiency . . . . . . . . . . . . . . 84

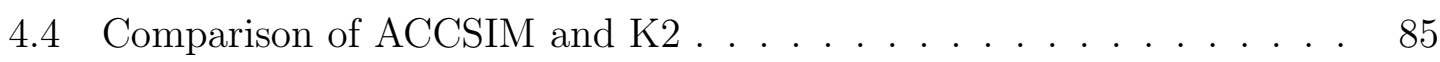

$\begin{array}{llr}5 & \text { Crystal Channeling Measurements } & 89\end{array}$

5.1 Data Acquisition and Analysis . . . . . . . . . . . . . . 90

5.2 Channeling Characteristics . . . . . . . . . . . . . 94

5.3 Crystal Collimation and Background Reduction . . . . . . . . . 103

6 Collimation 111

6.1 PIN Diode Calibration . . . . . . . . . . . . . . . . . . 111

6.2 Beam Profile Measurements . . . . . . . . . . . . . . . . 114

6.3 Collimator Efficiency . . . . . . . . . . . . . . . . . 118

6.4 Longitudinal Halo Removal . . . . . . . . . . . . . . . . . 127

6.5 Diffusion Studies . . . . . . . . . . . . . . . . . 130

6.6 Frequency Analysis of Beam Losses . . . . . . . . . . . . . . 135

7 Upgrade of the RHIC Collimation System 139

7.1 Design . . . . . . . . . . . . . . . . . . . . . . 139

7.2 Simulation . . . . . . . . . . . . . . . . . . 142

7.3 Controls Software . . . . . . . . . . . . . . . . . . 145

7.4 Collimator Performance . . . . . . . . . . . . . 147

7.5 Future Directions . . . . . . . . . . . . . . . 151

$\begin{array}{ll}\text { Conclusion } & 153\end{array}$ 
A Constructing the Aperture Model 


\section{List of Figures}

1.1 The BNL Accelerator Complex . . . . . . . . . . . . . . 3

1.2 Frenet-Serret Coordinate System . . . . . . . . . . . . 6

2.1 Silicon Unit Cell . . . . . . . . . . . . . . . . . . . . . . . 22

$2.2\{110\}$ Silicon Lattice Planes . . . . . . . . . . . . . . . 22

2.3 Interplanar Potential $\ldots \ldots \ldots \ldots \ldots \ldots$

2.4 Geometry used to Calculate Crystal Acceptance . . . . . . . . . . . 29

2.5 Simple Collimation System . . . . . . . . . . . . . . . 36

2.6 Collimator Placement vs. Phase Advance . . . . . . . . . . . . . 38

2.7 Momentum Collimation Aperture . . . . . . . . . . . . 40

2.8 Beam Distribution $\ldots \ldots \ldots \ldots \ldots \ldots \ldots$

2.9 Width of Beam Distribution on Crystal . . . . . . . . . . 50

3.1 RHIC Collimator Configuration . . . . . . . . . . . 52

3.2 The RHIC Crystal . . . . . . . . . . . . . . . . . . 53

3.3 Effect of the Miscut Angle . . . . . . . . . . . . . . . 54

3.4 Crystal Collimator Vessel . . . . . . . . . . . . . . 55

3.5 Section View of the Crystal Vessel . . . . . . . . . . . . 55

3.6 Calibration of the LVDT $\ldots \ldots \ldots \ldots \ldots \ldots$

3.7 Comparison of Laser Spot Projections . . . . . . . . . . . . . 58 
3.8 Typical Laser Projection with Fit . . . . . . . . . . . . . . . 59

3.9 Calibration of Laser Left Edge to Centroid Pixel Number . . . . . . . 59

3.10 Copper Scraper Vessel . . . . . . . . . . . . . . . . . . . . . . 60

3.11 PIN Diode Block Diagram . . . . . . . . . . . . . . . . . 61

3.12 Hodoscope Geometry . . . . . . . . . . . . . . . . . . . . . . . 62

3.13 Circuit for Hodoscope . . . . . . . . . . . . . . . 63

$3.14 \beta$ Functions and Dispersion Function near the Crystal Collimator . . 65

3.15 Blue Scraper PET Page . . . . . . . . . . . . . . . . 66

3.16 Crystal Collimator Control Program . . . . . . . . . . . . . . 67

3.17 Scraper Control Program . . . . . . . . . . . . . . . . . 68

3.18 Effect of the Copper Scraper on the Beam . . . . . . . . . . . . . . 70

4.1 Simulation of Crystal Channeling . . . . . . . . . . . . . . . 74

4.2 UAL Architecture . . . . . . . . . . . . . . . 76

$4.3 \mathrm{UAL} / \mathrm{ROOT}$ Architecture . . . . . . . . . . . . . . . . . 77

4.4 Flow Chart of scan Simulation . . . . . . . . . . . . . . . . . . . 78

4.5 Typical Input Distribution for Crystal Simulations . . . . . . . . . . . 79

4.6 Simulation of a Crystal Scan . . . . . . . . . . . . . . . 81

4.7 Multiple Turn Channeling Simulation . . . . . . . . . . . . . . 82

4.8 Simulation of the Effect of Changing $\alpha$ on Channeling . . . . . . . . . 82

4.9 Comparison of Simulations in Figure 4.8 to Theory . . . . . . . . . . 83

4.10 Comparison of One Turn Simulation to Theory . . . . . . . . . . 83

4.11 Simulation of Loss Locations in RHIC Blue Ring . . . . . . . . . . . . 84

4.12 Particle Distribution used to Compare ACCSIM and K2 . . . . . . . 86

4.13 The Effects of Scattering in K2 and ACCSIM . . . . . . . . . 86

4.14 Comparison of Particle Loss Simulations with ACCSIM and K2 . . 87 
5.1 Typical Crystal Scan . . . . . . . . . . . . . . . . . . . . 91

5.2 Data from Figure 5.1 after Analysis . . . . . . . . . . . . . . . . 92

$5.3 \quad$ Hodoscope Data during Fill $01547 \ldots \ldots$. . . . . . . . . 93

5.4 Averaged Downstream PIN Diode Rate during an Angular Scan . . . 94

5.5 Comparison of Simulations with the Data from Fill $1547 \ldots \ldots$. . . 95

5.6 Graph of the Channeling Dip vs. Crystal Location . . . . . . . . . . 97

5.7 Simulated Particle Distribution at the Scraper Location . . . . . . . 103

5.8 PIN Diode Rates when the Scraper is Moved Relative to the Crystal . 104

5.9 STAR CTB Signal during Four Crystal Scans _ . . . . . . . . . 106

5.10 Selected Background Signals from the RHIC Experiments . . . . . . . 107

5.11 STAR Yellow Halo Signal during Crystal Collimation . . . . . . . . . 109

5.12 Change in STAR Background vs. Relative Normalized Scraper Position109

6.1 PIN Diode Rate and Beam Current . . . . . . . . . . . . . . . . 112

6.2 Beam Losses vs. PIN Diode Rate for Two Fills . . . . . . . . . . . . . 113

6.3 PIN Diode Calibration . . . . . . . . . . . . . . . . . . . 113

6.4 Data from the Yellow Ring with Gold for Fill 03254 . . . . . . . . 117

6.5 Data from the Blue Ring with Protons for Fill 02233 . . . . . . . 117

6.6 Losses Around the RHIC Ring on Ramp . . . . . . . . . . . . . . 119

6.7 Experimental Backgrounds during Fill 01759 . . . . . . . . . . 120

6.8 Experimental Backgrounds during Fill 03094 . . . . . . . . . . . . 122

6.9 Loss Pattern at Injection for Fill 03254 in the Blue Ring . . . . . . . 124

6.10 Actual and Simulated Loss Pattern for Fill 03254 in the Blue Ring . . 125

6.11 Actual and Simulated Loss Pattern for Fill 03750 in the Blue Ring . . 126

6.12 Location of the Tunemeter Kickers and Scrapers in RHIC . . . . . . 128

6.13 The GapCleaner Program ． . . . . . . . . . . . . . . . . . . 129 
6.14 Gap Cleaning during Fill $04471 \ldots \ldots \ldots$

6.15 Effect of the Scraper Motion on Beam Loss Rates with Fit . . . . . 133

6.16 Reconstructed Diffusion Coefficient for Fill 02797 . . . . . . . . . . 133

6.17 Blue PIN Diode Rate and FFT during Fill 01020 . . . . . . . . . . 136

6.18 Effect of the Booster/AGS Supercycle on the PIN Diode Rate . . . . 137

6.19 Pin Diode Rates when the Crystal Collimator Touches the Beam . . . 137

7.1 Suggested Collimation System Upgrade . . . . . . . . . . . . . . 140

7.2 Upgraded RHIC Collimation System _ . . . . . . . . . . . . . 141

7.3 Secondary Scrapers . . . . . . . . . . . . . . . . . . . 142

7.4 Input Distribution used in Simulations . . . . . . . . . . . . . 143

7.5 Simulation of Particles $10 \mathrm{~m}$ Downstream of the Primary Scraper . . 143

7.6 Simulation of the Fraction of Intercepted Particles . . . . . . . . . 144

7.7 The New Collimator Control Program _ . . . . . . . . . . . 145

7.8 Blue Experiment Background during Fill $04854 \ldots \ldots$. . . . . 147

7.9 Blue Experiment Background during Fill $04436 \ldots \ldots \ldots$

7.10 Loss Pattern for Fill $04854 \ldots \ldots$. . . . . . . . . . . 149

7.11 Actual and Simulated Loss Pattern for Fill 04854 in the Blue Ring . . 150

A.1 Example of Warm Section Components List . . . . . . . . . . . 164 


\section{List of Tables}

1.1 Summary of RHIC Parameters . . . . . . . . . . . . . . . . . . 4

1.2 Summary of RHIC Runs to Date . . . . . . . . . . . . 5

2.1 Silicon Lattice Parameters . . . . . . . . . . . . . . . . . . . . 22

2.2 Silicon Channeling Parameters . . . . . . . . . . . . . 25

2.3 Design Horizontal Beam Parameters at Crystal for Various $\beta_{P H E N I X}^{*} \quad 49$

3.1 Design Twiss Parameters at Collimators . . . . . . . . . . . 64

5.1 Synopsis of Channeling Data . . . . . . . . . . . . . . . 90

5.2 Comparison of Selected Data Sets to Simulations . . . . . . . . . . 96

5.3 Comparison of Measured $\sigma_{x x^{\prime}} / \sigma_{x}^{2}$ to Model . . . . . . . . . . . . . 98

5.4 Comparison of Measured $\sigma_{x^{\prime}}\left(x_{0}\right)$ to Model . . . . . . . . . . . . . . . 99

5.5 Comparison of Various Models of Expected Channeling Efficiency . . 100

5.6 Measured Channeling Efficiency . . . . . . . . . . . . . . . 101

5.7 Measurements of the Crystal Bend Angle . . . . . . . . . . . . . 102

5.8 Phase Advance Between Experiments and Crystal . . . . . . . . . . 108

6.1 Beam Profile Data Sets . . . . . . . . . . . . . . . 116

6.2 Comparison of Collimator and IPM Horizontal Emittance Measurement117

6.3 Experimental Detectors used for Background Measurement . . . . . . 121 
6.4 Background Reduction in FY2003 Runs . . . . . . . . . . . . . . . 123

6.5 Fit of the Diffusion Coefficient . . . . . . . . . . . . . . . . . 134

7.1 Approximate Phase Advance between Collimators . . . . . . . . . . . 141

7.2 Background Reduction in FY2004 Run . . . . . . . . . . . . . . . . . 149 


\section{Acknowledgments}

Acknowledging the people who helped you along the way to your thesis is a tricky task. Inevitably, there is someone missing from the list. To minimize this risk, I will go in some sort of chronological order.

In the beginning, God created the universe. I have to thank Him. After He invented the laws of nature, He created the UHEC (Ultra Huge Everything Collider) from which we all come. I thank Him for making me a creature in the universe, and for giving me the skills to study it.

My parents of course had something to do with my being here. They made many sacrifices to give me a proper Catholic education and the freedom to choose my area of study. They are always there for me and have bent over backwards numerous times for me. Thanks Mom and Dad.

Of course, I cannot forget my sister. She makes sure that I never forget that I am a nerd, and will always remain that way. I only wish she was wrong....

My lovely wife, Lisa, is a constant source of encouragement and support. She was understanding when I would come home at 9am after 24 hours at the lab and would fall asleep. She enjoyed talking about the work that I do. Although she wouldn't understand it all, she knows enough to dazzle her friends. Lisa, this thesis is for you.

At this point, I will thank my in laws, Mom and Dad Gorycki. After I passed 
the $3^{\text {rd }}$ degree before I could date my wife, they treated me like their own son. I cannot forget my brother-in-law Mark, the skinniest guy I know and yet McDonald's owes much of their existence to him alone!

Hal Metcalf and Tom Hemmick were my undergraduate advisers. Hal taught me that it is important to have physical intuition. Tom introduced me to "big physics" and the importance of "writing EVERYTHING down".

I worked for a year or so with Gene Sprouse's group. I had a great time working in his lab. Thank you Gene, Luis, Matt, Josh, Seth, Joe, Wade, and Edwardo.

You owe a lot to your thesis adviser. Steve introduced me to accelerator physics. His constant questioning honed my understanding. I knew that if I could convince Steve of the physics involved, then I understood the subject.

Angelika was just as much an adviser to me as Steve and is great to work with. As busy as she was, she always had time to help me out and bounce ideas off of. Even when she was Scheduling Physicist, she could always find the time to help me. She also read enough manuscripts of this thesis to be able to recite it in her sleep!

Todd Satogata always had time to help me with my computer problems and my endless questions and nagging about the machine optics. Nikolay Malitsky was a great help with my simulation efforts. Nick D'Imperio was always a constant friend. Teaching with Waldo at USPAS was a pleasure. Thanks to the whole AP group and the entire C-AD department, it was a pleasure to work with all of you.

Special thanks to Lee Hammons, who made the fantastic Crystal Collimator control program. He is a great friend and an excellent co-worker. He made my life much easier. I'd also like to thank the rest of the operations group. As much as it pained them to let me use the collimators, I would like to say, "I never dumped a store!"

There were a number of engineers and technical staff that worked on the crystal 
collimator, Dave Gassner, Gary McIntyre, "Mr. Ed" Ulrich, Joe Saetta, and Frank Karl, and I'm sure there are others of which I am unaware.

Of course, there are my office mates Vahid, Javier, Ubaldo, Rama, and Jay. It was a pleasure to share an office with them. Always with the insight and humorous conversions, be it physics or casual conversation. Ram, where is my dollar??

I cannot leave out our Russian collaborators, Valery Biryukov, Yuri Chesnokov, and Victor Terehkov. They provided us with the crystals, and theoretical support when it was needed.

Everyone knows that the administrative staff makes the world go 'round. I'd like to thank Mary Campell, Anna Petaway, Marion Heimerle, Gladys Blas, and Dolores Collins at BNL and Pam Burris, Maria Hofer, Elaine Larsen, Sara Lutterbie, Pat Peiliker, and Diane Siegel at Stony Brook. Thank you for your help and guidance, keeping me clear of all of the administrative pitfalls.

I have been spoiled in my travels through Stony Brook and BNL. If I left anyone out, I'm sorry. 


\section{Chapter 1}

\section{Introduction}

Crystal channeling is a phenomenon in which the crystal lattice is used to guide ions through a crystal. Stark was the first to realize the possibility of crystal channeling in 1912 [1]. In the 1960s, computer simulations showed that ions with low angles with respect to low order planes in crystals would have long ranges [2]. Experiments also showed a variety of effects that depended on the crystal orientation [3]. Jens Lindhard was the first to provide an analytical theory of particle channeling in 1965 $[4]$.

Tsyganov proposed using bent crystals for bending particle beams in 1976 [5]. Particle deflection was first seen at Dubna in 1978 [6]. Shortly after, it was measured at CERN [7]. Channeling experiments at Fermilab in the early 1980's have seen channeling with proton energies up to $800 \mathrm{GeV}$ [8]. In 1989 IHEP, Protvino started doing experiments with crystal channeling and focusing [9]. Experiments have been carried out at these laboratories to the present [10].

Bent crystals provide a novel way to collimate beams. By replacing the primary collimator with a bent crystal, it is possible to kick the halo away from the beam core to efficiently deposit it into a secondary collimator. The secondary collimator 
can be placed at an appropriate location to catch all of the channeled beam from the crystal, and be far enough away from the circulating beam so as not to disturb the core. If the crystal has a high channeling efficiency, the collimation efficiency can be greatly improved as compared to a conventional two stage collimation system. The idea to use a bent crystal for collimation in RHIC was first proposed in 1997 [11].

Another proposed use of bent crystals at Brookhaven is at the NASA Space Radiation Laboratory (NSRL) [12]. At NSRL, some experiments require low flux, micron sized, heavy ion beams to irradiate single cells. The purpose is to look for intracellular and neighboring cell effects due to radiation. To achieve such a beam, a small low flux initial beam that is constant in position required. A micron sized bent crystal can reduce the beam flux, while providing the required beam size and position stability.

In this thesis, we explore the use of bent crystals for beam collimation in RHIC. The remainder of this chapter discusses the relevant RHIC parameters and beam dynamics needed to understand collimation. Chapter 2 covers collimation principles, crystal channeling, and crystal collimation. The specifics of the RHIC Crystal Collimation system are described in Chapter 3. Simulations of the RHIC Collimation system are examined in Chapter 4. Chapter 5 discusses experiments with and the use of the crystal collimator. The experiments done with the conventional copper scrapers at RHIC are recounted in Chapter 6. Chapter 7 details the redesigned collimation system for the RHIC.

\subsection{Relativistic Heavy Ion Collider}

The Relativistic Heavy Ion Collider (RHIC) at Brookhaven National Laboratory was built to study the physics of the Quark-Gluon Plasma and proton spin physics. 
RHIC is a $3833 \mathrm{~m}$ circumference machine consisting of two superconducting rings that intersect at six interaction regions. The two rings are referred to as "blue" and "yellow". The beam circulates in the clockwise direction in the blue ring and counterclockwise in the yellow ring. RHIC is capable of accelerating a variety of species from protons to fully stripped gold ions. The maximum beam energy is $100 \mathrm{GeV} / \mathrm{u}$ for gold and $250 \mathrm{GeV}$ for protons [13]. For all RHIC runs to date, the proton beam energy has been limited to $100 \mathrm{GeV}$ by request of the RHIC experiments.

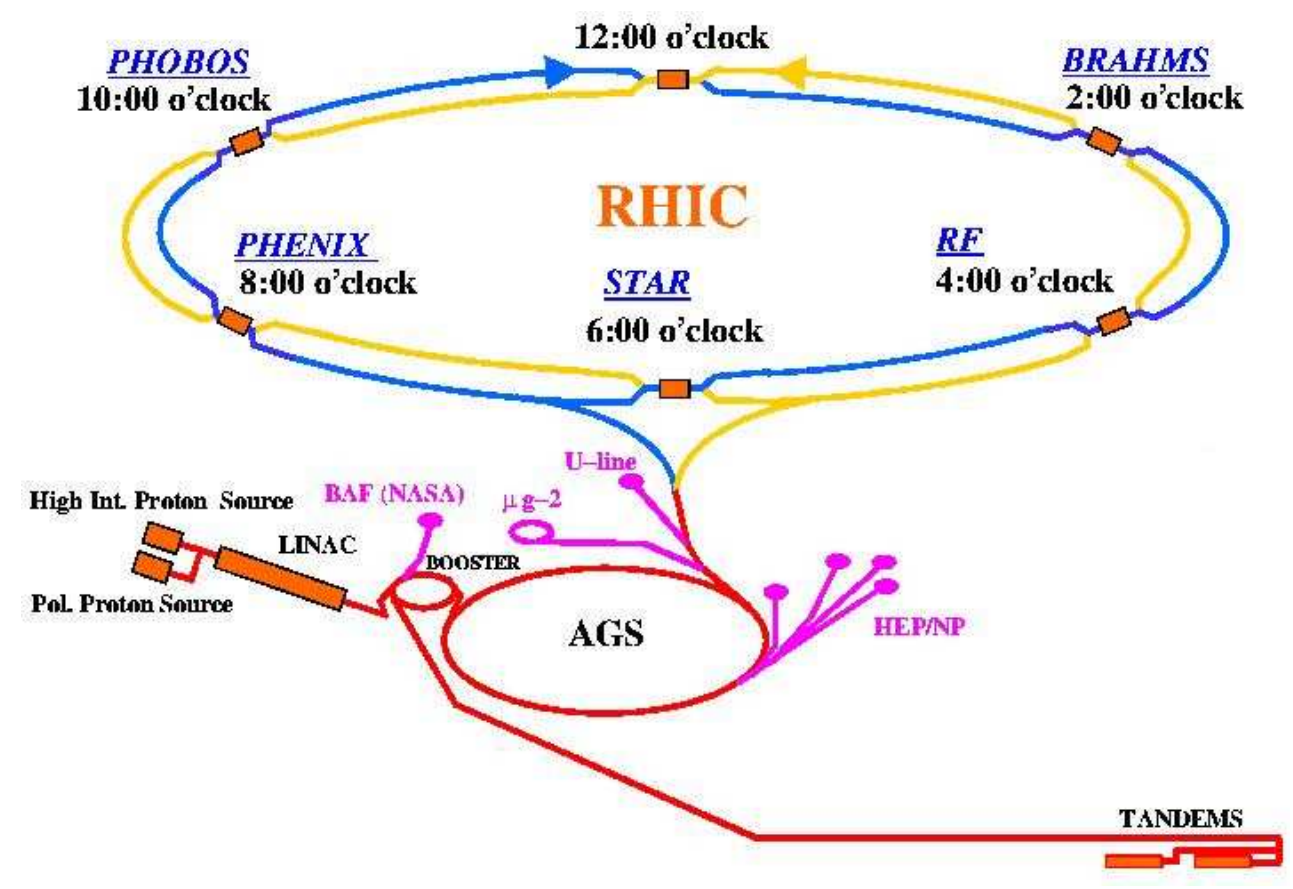

Figure 1.1: The BNL Accelerator Complex, showing injectors and low energy beamlines.

RHIC serves five experiments, STAR, PHENIX, BRAHMS, PHOBOS, and PP2PP. The Solenoidal Tracker at RHIC (STAR) is one of two large detectors at RHIC and is located in the IP6 position of RHIC. It specializes in tracking the thousands of particles produced by each ion collision at RHIC. The goal of STAR is to obtain a fundamental understanding of the structure of interactions between hadrons [14]. The Pioneering High Energy Nuclear Interaction eXperiment (PHENIX) detec- 
Table 1.1: Summary of RHIC Parameters

\begin{tabular}{|c|c|c|}
\hline & $\mathrm{Au}$ & $\mathrm{p}$ \\
\hline Circumference & \multicolumn{2}{|c|}{$3833 \mathrm{~m}$} \\
Species & \multicolumn{2}{|c|}{$\mathrm{p}$ to $\mathrm{Au}$} \\
No. of Bunches & \multicolumn{2}{|c|}{$55 / 110$} \\
Store Length & \multicolumn{2}{|c|}{4 hours } \\
RMS momentum spread & \multicolumn{2}{|c|}{$0.13 \%$} \\
RMS bunch length & \multicolumn{2}{|c|}{$0.19 \mathrm{~m}$} \\
Energy & $100 \mathrm{GeV} / \mathrm{u}$ & $250 \mathrm{GeV}$ \\
Intensity & $10^{9}$ ions & $10^{11}$ ions \\
95\% normalized Emittance & $10 \pi \mathrm{mm}-\mathrm{mrad}^{2}$ & $20 \pi \mathrm{mm}-\mathrm{mrad}^{2}$ \\
Luminosity & $2 \times 10^{26} \mathrm{~cm}^{2} \mathrm{~s}^{-1}$ & $2 \times 10^{32} \mathrm{~cm}^{2} \mathrm{~s}^{-1}$ \\
\hline
\end{tabular}

tor is the other large detector, located in the IP8 position of RHIC. It is designed specifically to measure direct probes of the collisions such as leptons and photons [15]. The PHOBOS experiment is one of two smaller detectors and is located in the IP10 position of RHIC. It is designed to examine and analyze a very large number of unselected collisions with the premise that rare events will be readily identified [16]. The Broad Range HAdron Magnetic Spectrometer (BRAHMS) is the other small detector at RHIC located in the IP2 position of RHIC. It has the goal of precise measurements of charged hadrons over a large range of rapidity an transverse momentum [17]. The PP2PP experiment shares the IP2 interaction region with BRAHMS. It's goal is to study proton-proton elastic scattering and therefore participates only in proton runs $[18]$.

The first physics run was in 2000 with gold beams. Subsequent runs have utilized the flexibility of RHIC, using gold and proton beams. The FY2003 run used deuteron beams in the blue ring with gold in the yellow ring. This is summarized in Table 1.2. Future runs will include light ion beams such as silicon or copper. 
Table 1.2: Summary of RHIC Runs to Date

\begin{tabular}{|c|c|c|c|c|}
\hline Year & Species & Energy & PHENIX $\beta^{*}$ & Integrated Luminosity \\
\hline 2000 & Gold & $70 \mathrm{GeV} / \mathrm{u}$ & $3 \mathrm{~m}$ & $7.3 \mu \mathrm{b}^{-1}$ (PHENIX) \\
2001 & Gold & $100 \mathrm{GeV} / \mathrm{u}$ & $5 \rightarrow 2 \rightarrow 1 \mathrm{~m}$ & $92.6 \mu \mathrm{b}^{-1}$ (PHENIX) \\
2002 & proton & $100 \mathrm{GeV}$ & $3 \mathrm{~m}$ & $100 \mathrm{nb}^{-1}$ (STAR) \\
2003 & Deuteron \& Gold & $100 \mathrm{GeV} / \mathrm{u}$ & $2 \mathrm{~m}$ & $27 \mathrm{nb}^{-1}$ (PHENIX) \\
2003 & proton & $100 \mathrm{GeV}$ & $2 \rightarrow 1 \mathrm{~m}$ & $2500 \mathrm{nb}^{-1}$ (STAR) \\
2004 & Gold & $100 \mathrm{GeV} / \mathrm{u}$ & $1 \mathrm{~m}$ & $1370 \mu \mathrm{b}^{-1}$ (PHENIX) \\
\hline
\end{tabular}

\subsection{Linear Beam Dynamics}

A detailed introduction to accelerator physics can be found in References [19] and [20]. The treatment in this section is taken from Reference [20].

\subsubsection{Equations of Motion}

Motion of a charged particle in an accelerator is governed by the Lorentz Force Law

$$
\frac{\mathrm{d} \vec{p}}{\mathrm{~d} t}=q(\vec{E}+\vec{v} \times \vec{B})
$$

where $\vec{p}$ is the relativistic momentum, $\vec{v}$ is the velocity, $\vec{E}$ and $\vec{B}$ are the electric and magnetic fields, and $q$ is the charge of the particle. In all modern accelerators, magnetic fields are used to steer and focus the beam while electric fields are used to accelerate and maintain the time structure of the beam. Since only the trans-

verse motion of particles is important for collimation, the electric field term will be neglected.

To describe the motion of the particles, it is useful to define a reference orbit and then use a set of coordinates that describe motion about that orbit. This is known as the Frenet-Serret coordinate system shown in Figure 1.2. The position of the reference particle is given by $\overrightarrow{r_{0}}$. Its longitudinal location is given by the coordinate 


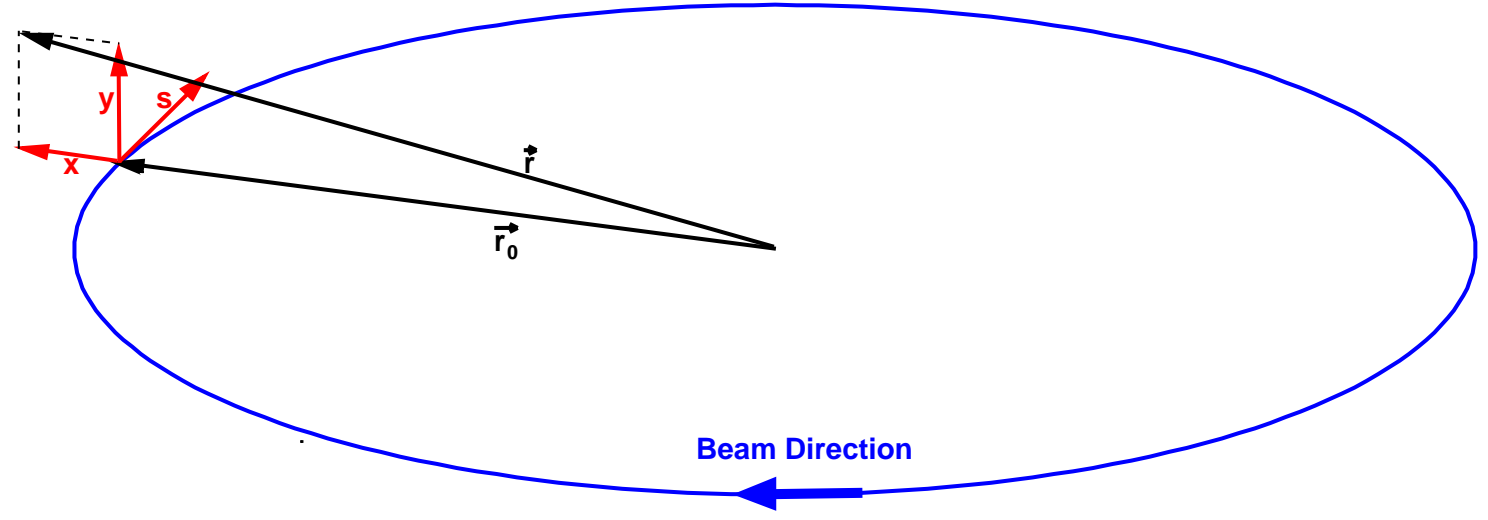

Figure 1.2: Frenet-Serret Coordinate System

$\vec{s}$. The transverse particle location is given by $\vec{r}=\overrightarrow{r_{0}}+x \hat{x}+y \hat{y}$.

Most accelerators are built in a plane so horizontal dipole fields can be ignored. The vertical dipole field defines the design orbit of the beam with a local bending radius of

$$
\rho=\frac{p}{q B}
$$

where $\rho$ is the local radius of curvature. The quantity $B \rho=p / q$ is known as the magnetic rigidity. Assuming that no longitudinal fields are present, the transverse equations of motion can be written as

$$
\begin{gathered}
x^{\prime \prime}(s)-\frac{\rho(s)+x(s)}{\rho(s)^{2}}=\frac{B_{y}(x, y, s)}{B \rho} \frac{p_{0}}{p}\left(1+\frac{x(s)}{\rho(s)}\right)^{2} \\
y^{\prime \prime}(s)=-\frac{B_{x}(x, y, s)}{B \rho} \frac{p_{0}}{p}\left(1+\frac{x(s)}{\rho(s)}\right)^{2}
\end{gathered}
$$

where $p$ is the particle momentum, $p_{0}$ is the momentum of the reference particle, $B_{x, y}$ are the $x$ and $y$ components of the magnetic field, and the prime denotes differentiation with respect to the $s$ coordinate.

The magnetic field can be expanded in terms of field multipoles. To first order 
these fields are

$$
\begin{aligned}
B_{y} & =-B_{0}+\frac{\partial B_{y}}{\partial x} x=-B_{0}+B_{1} x \\
B_{x} & =\frac{\partial B_{y}}{\partial x} y=-B_{1} y
\end{aligned}
$$

where $B_{0}$ and $B_{1}$ are the dipole and quadrupole coefficients of the field. With these fields, assuming that the particle momentum is the reference momentum, the transverse equations of motion become

$$
\begin{gathered}
x^{\prime \prime}+K_{x}(s) x=0 \quad, \quad K_{x}(s)=\frac{1}{\rho(s)^{2}}-\frac{B_{1}(s)}{B \rho} \\
y^{\prime \prime}+K_{y}(s) y=0 \quad, \quad K_{y}(s)=\frac{B_{1}(s)}{B \rho} .
\end{gathered}
$$

Variations in particle momentum will be addressed in Section 1.2.3. It is worth noting that in a circular machine, $K_{x, y}(s)$ are at least periodic in the circumference, $C$. However, often accelerators are built with a higher periodicity to ease design and operation.

\subsubsection{Solution of the Equations of Motion}

Letting $v$ stand for either $x$ or $y$, and $K_{v}(s)$ equal the corresponding focusing function, Equations 1.6a and 1.6b become

$$
v^{\prime \prime}+K_{v}(s) v=0
$$

This is Hill's equation. It is useful to think of Hill's equation as the harmonic oscillator equation with a time dependent spring constant. A solution to Hill's equation can 
be written as

$$
v(s)=A \sqrt{\beta_{v}(s)} \sin \left(\psi_{v}(s)+\psi_{0}\right) .
$$

$\beta(s)$ is the amplitude modulation of the oscillation and is referred to as the $\beta$ function or the envelope function. $\psi(s)$ is the phase advance. Inserting this into Hill's equation yields

$$
\begin{gathered}
\frac{\beta^{\prime \prime}(s)}{2}+K(s) \beta(s)-\frac{1}{\beta(s)}\left[1+\frac{\beta^{\prime}(s)^{2}}{4}\right]=0 \\
\psi(s)=\int_{0}^{s} \frac{\mathrm{d} s^{\prime}}{\beta\left(s^{\prime}\right)} .
\end{gathered}
$$

The subscript $v$ has been dropped. Since $K(s)$ is periodic, it is only necessary to solve the equations over one period. Equation $1.9 \mathrm{~b}$ shows that $\psi(s)$ and $\beta(s)$ are related and that another interpretation for $\beta(s)$ is the local wavelength of oscillation. The motion described by Equation 1.8 is referred to as the betatron motion. The value of the $\beta$ function at the center of an experiment is known as $\beta^{*}$.

It is convenient to define, $\nu_{v}$, of the number of oscillations per revolution of the accelerator.

$$
\nu_{v}=\frac{1}{2 \pi} \psi_{v}(C)=\frac{1}{2 \pi} \int_{s_{0}}^{s_{0}+C} \frac{\mathrm{d} s}{\beta_{v}(s)}
$$

This is known as the betatron tune. A resonance can develop if the tune is a low order rational number, this will lead to beam loss [20].

It is convenient to define two quantities that are related to the $\beta$ function

$$
\begin{aligned}
& \alpha(s)=-\frac{\beta^{\prime}(s)}{2} \\
& \gamma(s)=\frac{1+\alpha(s)^{2}}{\beta(s)} .
\end{aligned}
$$

$\alpha, \beta$, and $\gamma$ are collectively known as the Courant-Snyder or Twiss parameters.

Often, the position and angle of a particle, $\left\{v, v^{\prime}\right\}$, are known at a specific 
location $s_{1}$ and $\left\{v, v^{\prime}\right\}$ are desired at a location $s_{2}$. For this purpose, it is useful to rewrite Equation 1.8 in the form

$$
v(s)=a \sqrt{\beta(s)} \sin \psi(s)+b \sqrt{\beta(s)} \cos \psi(s) .
$$

Solving for $\{a, b\}$ in terms of $\left\{v\left(s_{1}\right), v^{\prime}\left(s_{1}\right)\right\}$, yields

$$
\begin{aligned}
& a=v\left(s_{1}\right)\left[\frac{\sin \psi\left(s_{1}\right)+\alpha\left(s_{1}\right) \cos \psi\left(s_{1}\right)}{\sqrt{\beta\left(s_{1}\right)}}\right]+v^{\prime}\left(s_{1}\right) \sqrt{\beta\left(s_{1}\right)} \cos \psi\left(s_{1}\right) \\
& b=v\left(s_{1}\right)\left[\frac{\cos \psi\left(s_{1}\right)-\alpha\left(s_{1}\right) \sin \psi\left(s_{1}\right)}{\sqrt{\beta\left(s_{1}\right)}}\right]-v^{\prime}\left(s_{1}\right) \sqrt{\beta\left(s_{1}\right)} \sin \psi\left(s_{1}\right) .
\end{aligned}
$$

Substituting Equations 1.14 into Equations 1.13 the solution for $\left\{v\left(s_{2}\right), v^{\prime}\left(s_{2}\right)\right\}$ in matrix form is found to be

$$
\left(\begin{array}{c}
v\left(s_{2}\right) \\
v^{\prime}\left(s_{2}\right)
\end{array}\right)=M\left(s_{2} \mid s_{1}\right)\left(\begin{array}{c}
v\left(s_{1}\right) \\
v^{\prime}\left(s_{1}\right)
\end{array}\right)
$$

where

$$
M\left(s_{2} \mid s_{1}\right)=\left(\begin{array}{cc}
\sqrt{\frac{\beta_{2}}{\beta_{1}}}\left(\cos \psi_{21}+\alpha_{1} \sin \psi_{21}\right) & \sqrt{\beta_{2} \beta_{1}} \sin \psi_{21} \\
\frac{-\left(1+\alpha_{1} \alpha_{2}\right)}{\sqrt{\beta_{2} \beta_{1}}} \sin \psi_{21}+\frac{\alpha_{1}-\alpha_{2}}{\sqrt{\beta_{2} \beta_{1}}} \cos \psi_{21} & \sqrt{\frac{\beta_{1}}{\beta_{2}}}\left(\cos \psi_{21}-\alpha_{2} \sin \psi_{21}\right)
\end{array}\right)
$$

is the transfer matrix between $s_{1}$ and $s_{2}$. The subscripts 1 and 2 denote that a specific variable is evaluated at $s_{1}$ and $s_{2}$, while $\psi_{21}$ is the phase advance between $s_{1}$ and $s_{2}$. It is easy to show that the transfer matrix between $s_{1}$ and $s_{3}$ is simply

$$
M\left(s_{3} \mid s_{1}\right)=M\left(s_{3} \mid s_{2}\right) M\left(s_{2} \mid s_{1}\right) .
$$

Using these matrices, one can trace a particle's trajectory through any linear 
lattice. A convenient matrix for a circular machine is the matrix for one full revolution starting from an arbitrary point $s$. This matrix is parameterized by

$$
M(s+C \mid s)=\left(\begin{array}{cc}
\cos 2 \pi \nu+\alpha \sin 2 \pi \nu & \beta \sin 2 \pi \nu \\
-\gamma \sin 2 \pi \nu & \cos 2 \pi \nu-\alpha \sin 2 \pi \nu
\end{array}\right)
$$

It is easy to see that Equations 1.18 is a special case of Equations 1.16.

It is convenient to introduce normalized coordinates $\left\{v_{n}, v_{n}^{\prime}\right\}$ by

$$
\left(\begin{array}{c}
v_{n} \\
v_{n}^{\prime}
\end{array}\right)=\left(\begin{array}{cc}
\frac{1}{\sqrt{\beta}} & 0 \\
\frac{\alpha}{\sqrt{\beta}} & \sqrt{\beta}
\end{array}\right)\left(\begin{array}{c}
v \\
v^{\prime}
\end{array}\right)
$$

In these coordinates, the transfer matrix of Equations 1.16 becomes

$$
M\left(s_{2} \mid s_{1}\right)=\left(\begin{array}{cc}
\cos \psi_{21} & \sin \psi_{21} \\
-\sin \psi_{21} & \cos \psi_{21}
\end{array}\right)
$$

which is the familiar rotation matrix. In this representation, the trajectory of $\left\{v_{n}, v_{n}^{\prime}\right\}$ is a circle of diameter

$$
2 J=\gamma v^{2}+2 \alpha v v^{\prime}+\beta v^{\prime 2} .
$$

The particle precesses about this circle by the angle $\psi(s)$ as it travels through the machine. The particle action, $J$, also known as the Courant-Snyder invariant, is a constant of the motion. 


\subsubsection{Dispersion}

To consider particles which are not at the design momentum $p_{0}$, Equation 1.6a needs to include the neglected momentum terms. It becomes

$$
x^{\prime \prime}+\left[\frac{1-\delta}{\rho(s)^{2}(1+\delta)}-\frac{B_{1}(s)}{B \rho(1+\delta)}\right] x=\frac{\delta}{\rho(s)(1+\delta)}
$$

where

$$
\delta=\frac{p-p_{0}}{p_{0}}
$$

is the momentum deviation. To solve Equation 1.22, it is useful to look for solutions of the form

$$
x(s)=x_{\beta}(s)+D(s) \delta
$$

where $D(s)$ is the dispersion function. Plugging this into Equation 1.22, and keeping only terms to first order in $\delta$ reveals

$$
\begin{gathered}
x_{\beta}^{\prime \prime}+\left[K_{x}(s)+\Delta K_{x}(s)\right] x_{\beta}=0 \\
D^{\prime \prime}+\left[K_{x}(s)+\Delta K_{x}(s)\right] D=\frac{1}{\rho(s)} \\
\Delta K_{x}(s)=\left[-\frac{2}{\rho(s)^{2}}+\frac{B_{1}(s)}{B \rho}\right] \delta .
\end{gathered}
$$

$\Delta K_{x}(s)$ is a chromatic perturbation to the focusing function from the momentum deviation. Since $\Delta K_{x} \ll K_{x}$, it will be neglected. Notice that Equation 1.25a is then identical to Equation 1.6a. If there are no vertical bends or skew elements Equation $1.6 \mathrm{~b}$ is unchanged. 
The solution of Equation $1.25 \mathrm{~b}$ is given by

$$
D(s)=\int_{0}^{s} \frac{\sqrt{\beta(s) \beta\left(s^{\prime}\right)}}{\rho\left(s^{\prime}\right)} \sin \left(\psi\left(s^{\prime}\right)-\psi(s)\right) \mathrm{d} s^{\prime}
$$

One can also consider a matrix solution to Equation 1.25b, by direct analogy to Equation 1.15. The matrix solution becomes

$$
\left(\begin{array}{l}
D\left(s_{2}\right) \\
D^{\prime}\left(s_{2}\right)
\end{array}\right)=M\left(s_{2} \mid s_{1}\right)\left(\begin{array}{c}
D\left(s_{1}\right) \\
D^{\prime}\left(s_{1}\right)
\end{array}\right)+\left(\begin{array}{c}
d \\
d^{\prime}
\end{array}\right)
$$

where $\bar{d}$ represents the effect of dipoles and is called the dispersion vector. Solving for $\bar{d}$ yields,

$$
\left(\begin{array}{c}
d \\
d^{\prime}
\end{array}\right)=\left(\begin{array}{c}
D\left(s_{2}\right)-D\left(s_{1}\right) M_{11}-D^{\prime}\left(s_{1}\right) M_{12} \\
D\left(s_{2}^{\prime}\right)-D\left(s_{1}\right) M_{21}-D^{\prime}\left(s_{1}\right) M_{22}
\end{array}\right)
$$

where $M_{i j}$ is the $\{i, j\}^{\text {th }}$ element of the matrix in Equation 1.16. The Equation 1.27 can be written in a more convenient form

$$
\left(\begin{array}{c}
D\left(s_{2}\right) \\
D^{\prime}\left(s_{2}\right) \\
1
\end{array}\right)=\left(\begin{array}{cc}
M\left(s_{2} \mid s_{1}\right) & \bar{d} \\
0 & 1
\end{array}\right)\left(\begin{array}{c}
D\left(s_{1}\right) \\
D^{\prime}\left(s_{1}\right) \\
1
\end{array}\right)
$$

Because the equation for betatron motion is the homogeneous form of the equation for the dispersion, and Equation 1.29 is the solution to the inhomogeneous equation, it is possible to use the matrix in Equation 1.29 to track particle motion by replacing $\left\{D, D^{\prime}, 1\right\}$ with $\left\{x, x^{\prime}, \delta\right\}$. In addition one can also define a normalized dispersion,

$$
\left(\begin{array}{l}
D_{n} \\
D_{n}^{\prime}
\end{array}\right)=\left(\begin{array}{cc}
\frac{1}{\sqrt{\beta}} & 0 \\
\frac{\alpha}{\sqrt{\beta}} & \sqrt{\beta}
\end{array}\right)\left(\begin{array}{c}
D \\
D^{\prime}
\end{array}\right) .
$$

In this representation, a particle with momentum deviation, $\delta$ and action $J$ will 
precess about a circle of radius $J$ that is offset from the origin by $\left\{D \delta, D^{\prime} \delta\right\}$.

\subsubsection{Constants of the Motion}

By taking the derivative of Equation 1.24, and using the identity

$$
\sin ^{2} x+\cos ^{2} x=1
$$

the invariant of the motion emerges.

$$
\begin{aligned}
2 A= & \frac{1}{\beta}\left\{(v-D \delta)^{2}+\left[v \alpha+v^{\prime} \beta-\left(D \alpha+D^{\prime} \beta\right) \delta\right]^{2}\right\} \\
= & \gamma v^{2}+2 \alpha v v^{\prime}+\beta v^{\prime 2} \\
& +\left(\gamma D^{2}+2 \alpha D D^{\prime}+\beta D^{\prime 2}\right) \delta^{2} \\
& -2\left[v\left(\gamma D+\alpha D^{\prime}\right)+v^{\prime}\left(\alpha D+\beta D^{\prime}\right)\right] \delta
\end{aligned}
$$

The first term of Equation 1.32 is the Courant-Snyder invariant. The coefficient in parenthesis in the second term is known as the $\langle\mathcal{H}\rangle$ function, which is invariant in locations without dipoles. In these locations, $1 / \rho=0$ and the equations for dispersion and betatron motion are identical. The third term couples the transverse coordinates and the momentum deviation. Like the second term, it is invariant in regions without dipoles. Even though the second and third terms vary in locations where $1 / \rho \neq 0, A$ is invariant everywhere.

Often the Courant-Snyder invariant is casually equated with the emittance, $\epsilon$. It is more proper to define the emittance as the average action of a bunch.

$$
\epsilon_{r m s}=2\langle J\rangle
$$


For a gaussian distributed beam, the rms beam size is given by the standard deviation,

$$
\sigma(s)=\sqrt{\epsilon_{r m s} \beta(s)}
$$

For RHIC, it has been chosen to use

$$
\epsilon=6 \pi \epsilon_{r m s}
$$

which is the known as the $95 \%$ emittance of the bunch. The advantage of this definition is that $95 \%$ of the beam particles have actions that are smaller than $2 \epsilon$, in contrast to $34 \%$ in the case of $\epsilon_{r m s}$.

The variables $\left\{v, v^{\prime}\right\}$ are not canonical conjugate. However $\left\{v, p_{v}\right\}$ are conjugate momentum and position. $p_{v}$ is related to $v^{\prime}$ by

$$
v^{\prime}=\frac{p_{v}}{p}=\frac{p_{v}}{\gamma_{r} \beta_{r} m c}
$$

where $\gamma_{r} \beta_{r}$ are the Lorentz factors. Under acceleration, $p_{z}$ changes causing $v^{\prime}$ to decrease as the beam is accelerated. This reduces $\epsilon$. Therefore it is useful to introduce a normalized emittance

$$
\epsilon_{n}=\gamma_{r} \beta_{r} \epsilon
$$

which is invariant under acceleration. In this way, the emittance at different energies can be compared. In terms of $\epsilon_{n}$ the rms beam size is given by

$$
\sigma=\sqrt{\frac{\epsilon_{n} \beta}{6 \pi \beta_{r} \gamma_{r}}} .
$$




\subsection{Need for collimation}

One can extend the analysis of the previous section to include other effects such as nonlinear magnetic elements (sextupoles, octopoles, etc.), magnetic field errors, beam-beam interactions, collective effects (intra-beam scattering, coupled bunch instabilities), synchrotron radiation, and effects of the environment on the beam (impedance, beam-gas scattering). Unfortunately, these effects are often nonlinear and simple analytic solutions do not exist except for special cases. Nevertheless, the cumulative effect of these interactions is important. In particular, these effects cause the Courant-Snyder invariant to change, usually increasing. This causes particles near the center of the beam to diffuse into the beam tails and cause a beam halo. These halo particles may eventually be lost and potentially causing detector background, equipment damage, reduced component lifetime, and excessive radiation in the accelerator tunnel. One detector at RHIC, PHOBOS, is very sensitive to damage from background radiation caused by beam losses because of a large number of silicon detectors. In addition, the superconducting magnets in RHIC are susceptible

to quenching if too much energy is dissipated in them from beam loss. It is the job of the collimation system to remove the halo and to restrict the area of the accelerator that is exposed to large amounts of radiation.

In addition to experiment and machine protection from slow losses, the collimation system has a role to play in machine protection from catastrophic beam loss. One such situation is an abort kicker misfire. If the abort kicker fires out of time with the abort gap, a bunch passing through the abort kicker during the rise time of the abort kicker will not receive the full kick to reach the beam dump. In the case of the Large Hadron Collider (LHC) such a bunch can destroy magnets [21]. It may be feasible to place a collimator at a location such that it will be hit in this scenario. 
The lower limit on RHIC emittance growth, and hence halo growth, is intrabeam scattering (IBS) [22]. The emittance growth from IBS arises from ion collisions between particles within the bunch. A detailed derivation of IBS growth rates is given in References [23, 24]. For bunched gaussian beams, the growth rates are approximately

$$
\begin{aligned}
& \frac{1}{\tau_{p}}=\left\langle A \frac{\sigma_{h}^{2}}{\sigma_{p}^{2}} \mathrm{f}\left(\frac{\sigma_{h}}{\gamma_{r}} \sqrt{\frac{\beta_{x}}{\epsilon_{x}}}, \frac{\sigma_{h}}{\gamma_{r}} \sqrt{\frac{\beta_{y}}{\epsilon_{y}}}, \sigma_{h} \beta_{r} \sqrt{\frac{2 d}{r_{o}}}\right)\right\rangle \\
& \frac{1}{\tau_{x}}=\left\langleA \left[\mathrm{f}\left(\frac{\gamma_{r}}{\sigma_{h}} \sqrt{\frac{\epsilon_{x}}{\beta_{x}}}, \sqrt{\frac{\beta_{y} \epsilon_{x}}{\beta_{x} \epsilon_{y}}}, \gamma_{r} \beta_{r} \sqrt{\frac{2 d}{r_{o}} \frac{\epsilon_{x}}{\beta_{x}}}\right)\right.\right. \\
& \left.\left.+\frac{D_{x}^{2} \sigma_{h}^{2}}{\beta_{x} \epsilon_{x}} \mathrm{f}\left(\frac{\sigma_{h}}{\gamma_{r}} \sqrt{\frac{\beta_{x}}{\epsilon_{x}}}, \frac{\sigma_{h}}{\gamma_{r}} \sqrt{\frac{\beta_{y}}{\epsilon_{y}}}, \sigma_{h} \beta_{r} \sqrt{\frac{2 d}{r_{o}}}\right)\right]\right\rangle \\
& \frac{1}{\tau_{y}}=\left\langleA \left[\mathrm{f}\left(\frac{\gamma_{r}}{\sigma_{h}} \sqrt{\frac{\epsilon_{y}}{\beta_{y}}}, \sqrt{\frac{\beta_{x} \epsilon_{y}}{\beta_{y} \epsilon_{x}}}, \gamma_{r} \beta_{r} \sqrt{\frac{2 d}{r_{o}} \frac{\epsilon_{y}}{\beta_{y}}}\right)\right.\right. \\
& \left.\left.+\frac{D_{y}{ }^{2} \sigma_{h}{ }^{2}}{\beta_{y} \epsilon_{y}} \mathrm{f}\left(\frac{\sigma_{h}}{\gamma_{r}} \sqrt{\frac{\beta_{x}}{\epsilon_{x}}}, \frac{\sigma_{h}}{\gamma_{r}} \sqrt{\frac{\beta_{y}}{\epsilon_{y}}}, \sigma_{h} \beta_{r} \sqrt{\frac{2 d}{r_{o}}}\right)\right]\right\rangle
\end{aligned}
$$

for the longitudinal and transverse coordinates respectively, with

$$
\begin{aligned}
A & =\frac{N_{b} c r_{o}{ }^{2}}{64 \pi^{2} \beta_{r}{ }^{3} \gamma_{r}{ }^{4} \epsilon_{x} \epsilon_{y} \sigma_{p} \sigma_{s}} \\
\frac{1}{\sigma_{h}{ }^{2}} & =\frac{1}{\sigma_{p}{ }^{2}}+\frac{D_{x}{ }^{2}}{\beta_{x} \epsilon_{x}}+\frac{D_{y}{ }^{2}}{\beta_{y} \epsilon_{y}}
\end{aligned}
$$

where $r_{o}$ is the classical particle radius, $N_{b}$ is the number of particles in the bunch, $\sigma_{p}$ is the rms momentum spread, $\sigma_{s}$ is the rms bunch length, $d$ is the smaller of $\sigma_{x}$ and $\sigma_{y}$, and the angle brackets denote an average around the ring. The function $f(a, b, q)$ 
is given by

$$
\begin{aligned}
f(a, b, q) & =8 \pi \int_{0}^{1}\left\{2 \ln \left[\frac{q}{2}\left(\frac{1}{P}+\frac{1}{Q}\right)\right]-0.577 \ldots\right\} \frac{1-3 u^{2}}{P Q} \mathrm{~d} u \\
P & =a^{2}+\left(1-a^{2}\right) u^{2} \\
Q & =b^{2}+\left(1-b^{2}\right) u^{2}
\end{aligned}
$$

It has been shown [25] that

$$
\bar{\epsilon}_{s}\left[\frac{1}{\gamma^{2}}-\left\langle\frac{D_{x}^{2}}{\beta_{x}^{2}}\right\rangle-\left\langle\frac{D_{y}^{2}}{\beta_{y}^{2}}\right\rangle\right]+\left\langle\frac{\bar{\epsilon}_{x}}{\beta_{x}}\right\rangle+\left\langle\frac{\bar{\epsilon}_{y}}{\beta_{y}}\right\rangle=\text { constant. }
$$

where $\epsilon_{s}$ is the longitudinal invariant and the overbar denotes an average over all particles. Below transition energy, all of the terms are positive, and an equilibrium can exist between $\epsilon_{s}, \epsilon_{x}$, and $\epsilon_{y}$. However, above transition the parenthesized term becomes negative and unlimited emittance growth can occur. This has the effect of pushing particles out of the RF buckets and increasing the transverse beam size. Because the classical particle radius increases as $Z^{2} / A$, IBS is a more important problem for heavy ions than it is for protons. Measurements of emittance growth at RHIC consistently show that the longitudinal emittance growth is consistent with IBS predictions, but the transverse emittances grow faster than expected [22, 26].

The beam-beam effect has a large effect on emittance growth in RHIC. The beam-beam effect is the effect of the electromagnetic field of one bunch influencing the motion of a bunch in the opposing beam. Because of the separated rings in RHIC, the beam-beam effect only occurs in the interaction regions. For gaussian beams, the 
force felt by a particle is given by [27]

$$
\begin{aligned}
F_{y}\left(x, y, \sigma_{x}, \sigma_{y}\right)+ & i F_{x}\left(x, y, \sigma_{x}, \sigma_{y}\right)=\sqrt{\frac{2 \pi}{\sigma_{x}^{2}-\sigma_{y}^{2}}}\left[\mathrm{~W}\left(\frac{x+i y}{\sqrt{2\left(\sigma_{x}^{2}-\sigma_{y}^{2}\right)}}\right)\right. \\
& \left.-\exp \left(-\frac{x^{2}}{2 \sigma_{x}^{2}}-\frac{y^{2}}{2 \sigma_{y}^{2}}\right) \mathrm{W}\left(\frac{\frac{\sigma_{y}}{\sigma_{x}} x+i \frac{\sigma_{x}}{\sigma_{y}} y}{\sqrt{2\left(\sigma_{x}^{2}-\sigma_{y}^{2}\right)}}\right)\right]
\end{aligned}
$$

where $W(z)$ is the complex error function

$$
W(z)=\mathrm{e}^{-z^{2}}\{1-\operatorname{Erf}(-i z)\}
$$

These nonlinear fields can drive particles from the center of the beam into the halo. Near the center of the bunch, the force leads to a tune shift per interaction point

$$
\Delta \nu=\frac{3 N_{b} r_{0}}{2 \pi \epsilon_{n}}
$$

for round hadron beams [28]. For RHIC, $\Delta \nu \approx 0.0023$ for proton beams and $\Delta \nu \approx$ 0.0074 for gold beams with nominal beam intensities and emittances [28]. At large amplitudes, the tune shift is different, leading to a tune spread of the beam. This can place particles near resonances and drive them to higher amplitudes. In addition, when the beams have high and approximately equal intensities, it is possible to coherently excite the beams through the beam-beam effect. This is the case for RHIC [28].

It was found during the FY2001 run that certain warm sections of the RHIC rings see an increase in pressure when high intensity beams are present [29]. The cause of these pressure rises is an electron cloud [30]. These pressure rises not only limit the intensity of beam, but they are a source of emittance growth. The ions in the beam will Coulomb scatter from the residual gas atoms. It can be shown that 
the emittance growth rate from beam gas interactions is given by [20]

$$
\frac{1}{\tau_{b g}[\mathrm{~h}]}=2.345 \frac{\gamma_{r}\langle\beta\rangle[\mathrm{m}]}{\beta_{r} \epsilon_{n}[\pi \mathrm{mm}-\mathrm{mrad}]}\left(\frac{Z}{p c[\mathrm{GeV}]}\right)^{2} \frac{P_{g}[\mathrm{nTorr}] A_{g}}{X_{0 g}\left[\mathrm{~g} / \mathrm{cm}^{2}\right]}
$$

where $Z$ is the beam charge state, $\langle\beta\rangle$ is the average $\beta$ function, and $P_{g}, A_{g}, X_{0 g}$ are the pressure, gram molecular weight, and radiation length of the residual gas.

As particles circulate in an accelerator, they are confined longitudinally in bunches by radio frequency $(\mathrm{RF})$ cavities. Longitudinal phase space regions that can hold a bunch are called RF buckets. It is possible for particles to migrate out of the RF bucket. In RHIC, the main mechanism for this is IBS [22]. These particles drift longitudinally in RHIC and eventually fill the space between bunches. Some of this beam fills the location in the bunch train left empty for the kicker rise time, the abort gap. This beam does not receive the fill kick from the abort kicker and will miss the beam dump. This can cause a magnet quench at high energy. By placing a collimator at a large normalized dispersion region, or by transversely exciting the beam within the abort gap, it is possible to remove this beam. This is discussed in Section 6.4.

It is possible to describe emittance growth in terms of the diffusion equation

$$
\frac{\partial}{\partial t} \rho(J, t)=\frac{\partial}{\partial J} D(J) \frac{\partial}{\partial J} \rho(J, t)
$$

where $\rho(J, t)$ is the particle distribution, and $D(J)$ is the diffusion coefficient. The diffusion coefficient is the mean squared action change per unit time

$$
D(J)=\frac{1}{2} \frac{\left\langle(\Delta J)^{2}\right\rangle}{\Delta t}
$$


which is related to the mean action growth rate [31]

$$
\frac{\langle\Delta J\rangle}{\Delta t}=\frac{\mathrm{d}}{\mathrm{d} J} D(J)
$$

It can be shown that $D(J)$ is related to the emittance growth rate by [32]

$$
D(J)=\frac{2 J}{\gamma_{r} \beta_{r}} \frac{\mathrm{d} \epsilon}{\mathrm{d} t}
$$

Using Equation 1.48, and 1.51 it is possible to simulate beam growth for a variety of conditions. In Reference [33], a method for measuring $D(J)$ using collimators is discussed. The results of these measurements at RHIC are discussed in Section 6.5.

Because of the cumulative effect of these and other phenomena, it is necessary to have a good collimation system in a collider. With increasing beam energy and intensity the role of the collimation system becomes increasing important, not only for background reduction but also for machine protection. 


\section{Chapter 2}

\section{Crystal Collimation}

In order to understand the usefulness of a material for use as a collimator, it is necessary to understand how the beam particles will interact with it. When ions pass through a material they interact with the electrons and nuclei that compose it. The effects on an ion from a crystal will be different than from an amorphous solid because of the symmetry of a crystal structure.

In this chapter, the phenomenon of crystal channeling will be discussed along with a discussion of the physics of beam collimation and how bent crystals can be applied to beam collimation.

\subsection{Crystal Channeling}

In crystal channeling, the crystal lattice is used to guide ions through a crystal. The symmetry of crystals allows for the scattering angles of incident ions with the lattice atoms to be correlated in such a way that the ion follows the planes of the crystal [9]. Because of this, the structure and purity of the crystal are important. For an introduction to general crystal structure see any introductory solid state physics 


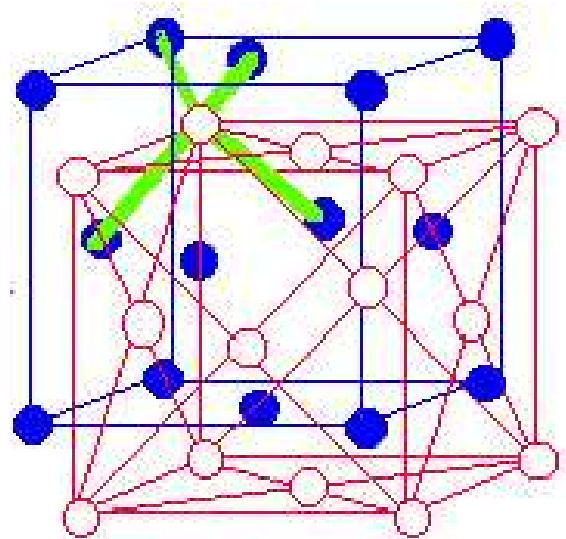

Figure 2.1: Silicon Unit Cell [35]. The green lines show the tetrahedron formed by lattice ions.

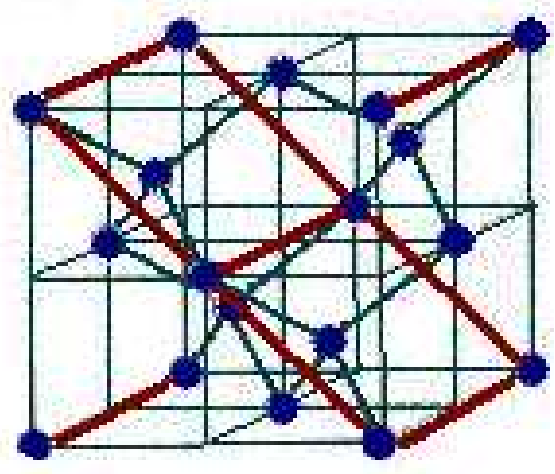

Figure 2.2: The $\{110\}$ plane group for a silicon lattice [36].

Table 2.1: Silicon Lattice Parameters from [9]

\begin{tabular}{|c|cccc|cccc|}
\hline Lattice & \multicolumn{3}{|c|}{ Axial Interatomic Spacing $[\AA]$} & \multicolumn{3}{c|}{ Interplanar Spacing $d_{p}[\AA]$} \\
Constant $[\AA]$ & $<100>$ & $<110>$ & $<111>\mathrm{L}$ & $<111>\mathrm{S}$ & $(100)$ & $(110)$ & $(111) \mathrm{L}$ & $(111) \mathrm{S}$ \\
\hline 5.43 & 5.43 & 3.85 & 1.54 & 4.59 & 1.36 & 1.92 & 0.78 & 2.35 \\
\hline
\end{tabular}

book [34].

\subsubsection{Crystal Structure}

Because of the availability of cheap, high quality silicon crystals, most experiments involving crystal channeling use silicon. The cell structure of silicon is shown in Figure 2.1. Silicon has a diamond lattice like carbon and germanium. A diamond cell is a face center cubic (fcc) lattice with atoms added near each corner so as to form a tetrahedron with the corner and two face atoms. The lattice can also be considered as two fcc lattices, one shifted from the other by $1 / 4$ of a body diagonal.

Table 2.1 shows some of the relevant Silicon lattice parameters. The crystals used in RHIC were grown to use the (110) planes to channel ions. Experiments at Dubna have used the (111) planes [37]. The (111) planes form two channels, often labeled (111)L and (111)S. Figure 2.2 shows the $\{110\}$ plane group. 


\subsubsection{Channeling}

The next two sections will closely follow the treatments given in References [9] and [38].

The potential due to a single atom on an ion passing near it is a complicated function of the nuclear and electron potentials. However, the internal structure of the atom is not important for determining the potential - only the gross atomic charge distribution is needed. An often used approximation that neglects all but the gross charge distribution is the Thomas - Fermi model [39]:

$$
V(r)=\frac{Z_{i o n} Z e^{2}}{4 \pi \epsilon_{o} r} \Phi\left(\frac{r}{a_{\mathrm{TF}}}\right)
$$

where $Z_{\text {ion }}$ is the charge of the passing ion, $Z$ is the nuclear charge, $a_{\mathrm{TF}}=0.8853 a_{B} Z^{1 / 3}$ is the Thomas - Fermi or screening length and represents the falloff length of the atomic electron cloud distribution, $a_{B}$ is the Bohr radius, and $\mathrm{r}$ is the distance from the atom. The shape of the potential from the atomic charge distribution is given by the dimensionless function $\Phi\left(r / a_{\mathrm{TF}}\right)$. The Moliére approximation for $\Phi\left(r / a_{\mathrm{TF}}\right)$ is often used,

$$
\Phi\left(\frac{r}{a_{\mathrm{TF}}}\right)=\sum_{i=1}^{3} \alpha_{i} \exp \left(\frac{-\beta_{i} r}{a_{\mathrm{TF}}}\right)
$$

where $\alpha_{i}=\{0.1,0.55,0.35\}$ and $\beta_{i}=\{6.0,1.2,0.3\}$ are dimensionless constants [9].

Assuming that an incident ion is traveling at a small angle relative to a crystal plane, one can average over the lattice sites and obtain a continuous potential for each plane.

$$
\mathrm{U}_{p l}(x)=N d_{p} \int_{-\infty}^{+\infty} \int_{-\infty}^{+\infty} V(x, y, z) \mathrm{d} y \mathrm{~d} z .
$$

where $N$ is the molar density, $d_{p}$ is the interplanar spacing, and $\{x, y, z\}$ are the coordinates relative to the crystal plane. For silicon, $N=12.06 \mathrm{~cm}^{-1}$. 
Because the nearest planes will contribute the most to the interplanar potential, the potential seen by an incident ion is well approximated by

$$
\mathrm{U}(x) \approx \mathrm{U}_{p l}\left(\frac{d_{p}}{2}-x\right)+\mathrm{U}_{p l}\left(\frac{d_{p}}{2}+x\right)-2 \mathrm{U}_{p l}\left(\frac{d_{p}}{2}\right)
$$

where $x=0$ is defined to be midway between the two planes, transverse to the planes. The last term subtracts out a constant so that $\mathrm{U}(0)=0$.

It is now possible to write the Hamiltonian for the ion's motion between the planes

$$
H=\sqrt{p_{x}^{2} c^{2}+p_{y}^{2} c^{2}+\frac{p_{z}^{2} c^{2}}{(1+x / R(z))^{2}}+m^{2} c^{4}}+\mathrm{U}(x)
$$

where $R(z)$ is the radius of curvature parallel in the $x z$ plane, parallel to the $x$ axis

Since it is assumed that the particle is moving almost parallel to the crystal planes, $p_{x}, p_{y} \ll p_{z} \approx p$. The Hamiltonian can then be rewritten as

$$
H=\frac{p_{x}^{2} c^{2}}{2 \sqrt{\frac{p_{z}^{2} c^{2}}{(1+x / R(z))^{2}}+m^{2} c^{4}}}+\frac{p_{y}^{2} c^{2}}{2 \sqrt{\frac{p_{z}^{2} c^{2}}{(1+x / R(z))^{2}}+m^{2} c^{4}}}+\mathrm{U}(x)+\sqrt{\frac{p_{z}^{2} c^{2}}{(1+x / R(z))^{2}}+m^{2} c^{4}}
$$

For the given curvature and potential the vertical momentum $p_{y}$ is conserved. We assume that $R(z)$ is constant and large compared to any crystal dimension, so $p_{z}$ is also conserved. Using Hamilton's equations and $\mathrm{d} t=\mathrm{d} z / v$, the transverse equation of motion becomes

$$
p v \frac{\mathrm{d}^{2} x}{\mathrm{~d} z^{2}}+\mathrm{U}^{\prime}(x)+\frac{p v}{R}=0
$$

where the prime denotes the derivative with respect to the $x$ coordinate.

The simplest case is channeling without crystal curvature, $p v / R=0$. Using the 
Table 2.2: Typical potentials and critical parameters for a silicon crystal (adopted from $[9])$.

\begin{tabular}{|r|c|c|c|}
\hline Parameter & $<110>$ & $<111>\mathrm{L}$ & $<111>\mathrm{S}$ \\
\hline $\mathrm{d}_{\mathrm{p}}[\AA]$ & 1.92 & 2.35 & 0.78 \\
\hline $\mathrm{U}\left(x_{c}\right)\left[\mathrm{eV} / Z_{\text {ion }}\right]$ & 16 & 19 & 4.2 \\
\hline $\mathrm{U}^{\prime}\left(x_{c}\right)\left[\mathrm{GeV} /\left(\mathrm{cm} Z_{\text {ion }}\right)\right]$ & 5.7 & 5.6 & 3.5 \\
\hline$\theta_{c}[\mu \mathrm{rad}]$ & 11 & 12 & 5.8 \\
\hline$R_{c}[\mathrm{~cm}]$ & 44 & 45 & 71 \\
\hline
\end{tabular}

$\theta_{c}$ and $R_{c}$ parameters assume fully stripped gold ions at an energy of $100 \mathrm{GeV} / \mathrm{u}$.

harmonic approximation, the interplanar potential is assumed to have the form

$$
\mathrm{U}(x)=U_{o}\left(\frac{2 x}{d_{p}}\right)^{2} .
$$

The solution to Equation 2.7 is

$$
\begin{gathered}
x(z)=\frac{d_{p}}{2} \sqrt{\frac{E_{T}}{U_{o}}} \sin \left(\frac{2 \pi z}{\lambda}+\phi_{0}\right) \\
\theta(z)=\frac{\mathrm{d} x}{\mathrm{~d} z}=\sqrt{\frac{2 E_{T}}{p v}} \cos \left(\frac{2 \pi z}{\lambda}+\phi_{0}\right)
\end{gathered}
$$

where the transverse energy and oscillation period are respectively,

$$
\begin{aligned}
E_{T} & =\frac{p_{x}^{2} c^{2}}{2 \sqrt{p_{z}^{2} c^{2}+m^{2} c^{4}}}+\mathrm{U}\left(x_{0}\right) \approx \frac{p v \theta_{0}^{2}}{2}+\mathrm{U}\left(x_{0}\right) \\
\lambda & =\pi d_{p} \sqrt{\frac{2 U_{o}}{p v}}
\end{aligned}
$$

where $x_{0}$ is the initial interplanar position and $\theta_{0}=p_{x 0} / p_{z}$ is the initial angle. The transverse energy is a constant of the motion. For RHIC energies and silicon crystals $\lambda \approx 30 \mu \mathrm{m}$.

For channeling to occur, it is required that $E_{T} \leq U_{o}$ or else the ions travel over 


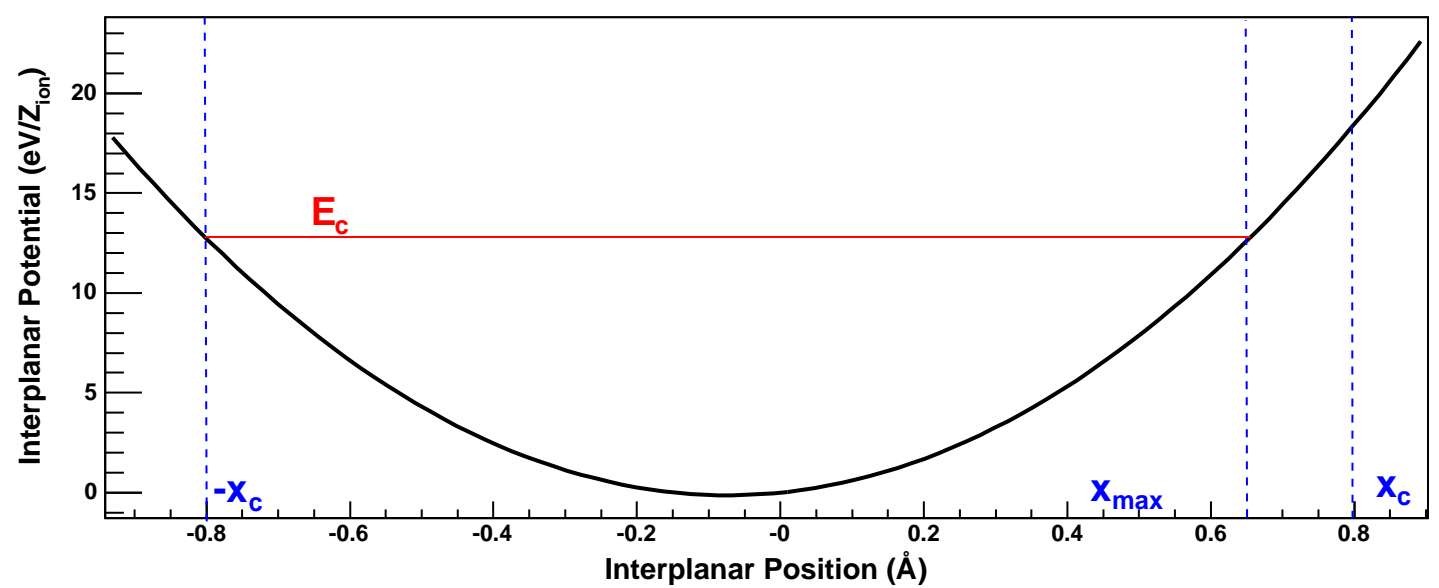

Figure 2.3: Interplanar Potential in a silicon crystal. The effect of curvature is exaggerated.

the potential provided by the atomic planes. This provides the condition

$$
\theta(z) \leq \theta_{L}=\sqrt{\frac{2 U_{o}}{p v}}
$$

where $\theta_{L}$ is the Lindhard angle. However, if an ion is inside the electron cloud of an atom, it could scatter and be dechanneled or a catastrophic nuclear interaction can occur. This can occur when $|x|>d_{p} / 2-a_{\mathrm{TF}}$. Thus, a critical distance is defined as

$$
x_{c}=\frac{d_{p}}{2}-a_{\mathrm{TF}}
$$

beyond which a nuclear interaction could happen. The critical angle is then defined to be

$$
\theta_{c}=\sqrt{\frac{2 E_{c}}{p v}}<\theta_{L}
$$

where $E_{c}=\mathrm{U}\left(x_{c}\right)$ is the critical transverse energy. At entrance angles below this angle, channeled particles will have a lower probability of encountering the lattice atoms. The angular acceptance of the crystal is $2 \theta_{c}$. For silicon crystals with fully 
stripped gold at $100 \mathrm{GeV} / \mathrm{u}$ or protons at $250 \mathrm{GeV}$, the critical angle is $11 \mu \mathrm{rad}$. For protons at $100 \mathrm{GeV}$, the critical angle is $19 \mu \mathrm{rad}$.

A straight crystal does not impart an overall angular kick to an ensemble of ions. In order to give a kick, it is necessary to bend the crystal planes. The simplest way to achieve this is to physically bend the crystal. Channeled ions will then be bent by an amount $\theta_{b}=L / R$ where $L$ is the crystal length.

For a bent crystal, the $p v / R$ term is nonzero. The effect of the the bend is to tilt the interplanar potential as is shown in Figure 2.3. Physically the reason for this is that on one side of the channel, the atoms are squeezed together slightly, and on the opposite side, the atoms are pulled apart. This leads to a greater repulsion from the squeezed side and less repulsion from the opposite side. There is a critical radius at which atoms on the spread side are pulled too far apart and the interplanar potential goes to zero on that side. This critical radius is given by

$$
R_{c}=\frac{p v}{U^{\prime}\left(x_{c}\right)}
$$

Typical values are given in Table 2.2.

The solution to Equation 2.7, including the bending term, is

$$
\begin{gathered}
x(z)=-x_{c} \frac{R_{c}}{R}+x_{c} \sqrt{\frac{E_{T}}{E_{c}}} \sin \left(\frac{2 \pi z}{\lambda}+\phi\right) \\
E_{T}=\frac{p v \theta_{0}^{2}}{2}+\mathrm{U}\left(x_{0}\right)+\frac{p v x_{0}}{R} .
\end{gathered}
$$

These are the solutions in Equation 2.9 displaced by an amount $-x_{c} R_{c} / R$. 
The critical energy and critical angle scale as

$$
\begin{aligned}
E_{c} & =E_{c, 0}\left(1-\frac{R_{c}}{R}\right)^{2} \\
\theta_{c} & =\theta_{c, 0}\left(1-\frac{R_{c}}{R}\right)
\end{aligned}
$$

where $E_{c, 0}$ and $\theta_{c, o}$ are the critical energy and angle for a straight crystal. For our case with $R \approx 10 \mathrm{~m}$, the correction $1-R_{c} / R \approx 0.96$.

\subsubsection{Crystal Acceptance}

The crystal acceptance is the ratio of the beam distribution at the entrance of the crystal to the phase space that is available for channeling. One can also think of it as the fraction of channeled particles in the limit of no dechanneling, and hence the maximum possible channeling efficiency. The crystal acceptance is

$$
A=\int_{x_{0}}^{x_{0}+\Delta x} \frac{\mathrm{d} x}{\Delta x} \int_{U_{e f f}(X)}^{E_{c}} \mathrm{~d} E_{T} \int_{-\infty}^{\infty} \mathrm{d} x^{\prime} \rho\left(x, x^{\prime}\right) \delta\left(\frac{p v \theta^{2}}{2}+U_{e f f}(X)-E_{T}\right)
$$

where $\rho\left(x, x^{\prime}\right)$ is the normalized beam density, $\theta=x^{\prime}-\theta_{\text {crystal }}-\theta_{\text {miscut }}$ is the angle between the crystal planes and the incident particle, $\theta_{\text {crystal }}$ is the angle between the crystal and beam direction, and $\theta_{\text {miscut }}$ is the angle between the crystal planes and the normal to the crystal face. This miscut angle will be discussed more fully in Section 3.1.1. The position inside of the crystal plane is $X$, the transverse coordinate of the beam is $x$, and $U_{\text {eff }}(X)$ is the interplanar potential including the term for the crystal curvature, $x_{0}$ is the location of the crystal edge relative to the closed orbit, $\Delta x$ is the crystal width, and $\delta$ is the Dirac delta function.

When a thin crystal is in the beam halo $x_{0} \gg \sigma_{x}$ and $\Delta x<\sigma_{x}$, then the beam 


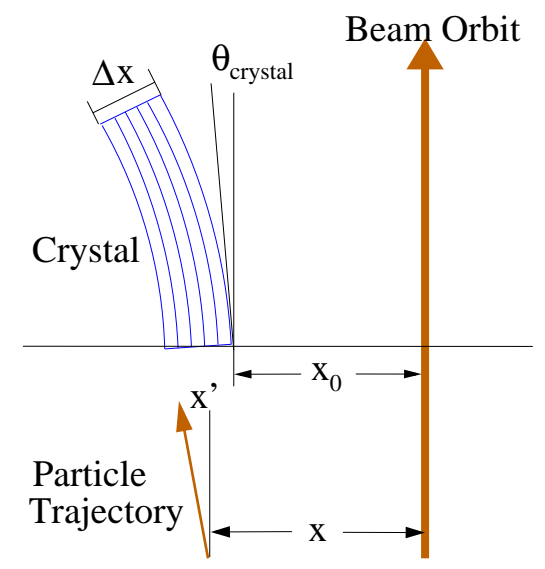

Figure 2.4: Geometry used to calculate crystal acceptance.

density is approximately a constant with position, $\rho\left(x, x^{\prime}\right) \approx \rho\left(x^{\prime}\right)$, over the crystal surface. The integral over $x$ becomes an integral over a single crystal channel and Equation 2.19 becomes

$$
A=\int_{-\frac{d_{p}}{2}}^{\frac{d_{p}}{2}} \frac{\mathrm{d} X}{d_{p}} \int_{U_{e f f}(X)}^{E_{c}} \mathrm{~d} E_{T} \int_{-\infty}^{\infty} \mathrm{d} x^{\prime} \rho\left(x^{\prime}\right) \delta\left(\frac{p v \theta^{2}}{2}+U_{e f f}(X)-E_{T}\right)
$$

To perform the angular integration, it is convenient to make a change of variables from $x^{\prime}$ to $x_{p}^{\prime}-\phi$ where $x_{p}^{\prime}$ is the center of the beam angular distribution on the crystal face and $\phi$ is the deviation from it. It is also convenient to make the transformation $\theta=\psi-\phi$ where $\psi=x_{p}^{\prime}-\theta_{\text {crystal }}-\theta_{\text {miscut }}$ is the angle between the crystal planes and the center of the beam angular distribution across the crystal face.

$$
A=\int_{-\frac{d_{p}}{2}}^{\frac{d_{p}}{2}} \frac{\mathrm{d} X}{d_{p}} \int_{U_{e f f}(X)}^{E_{c}} \mathrm{~d} E_{T} \int_{-\infty}^{\infty} \mathrm{d}(-\phi) \rho\left(x_{p}^{\prime}-\phi\right) \delta\left(\frac{p v(\psi-\phi)^{2}}{2}+U_{\text {eff }}(X)-E_{T}\right)
$$


To integrate over the delta function, one needs to make use of

$$
\delta(g(\theta))=\sum_{\left\{\theta_{i}\right\}} \frac{1}{\left|g^{\prime}\left(\theta_{i}\right)\right|} \delta\left(\theta_{i}\right), \quad \text { where } \theta_{i} \text { is defined as } \quad g\left(\theta_{i}\right)=0 \text {. }
$$

The acceptance becomes

$$
A=\sum_{ \pm} \int_{-\frac{d_{p}}{2}}^{\frac{d_{p}}{2}} \frac{\mathrm{d} X}{d_{p}} \int_{U_{e f f}(X)}^{E_{c}} \mathrm{~d} E_{T} \frac{\rho\left(x_{p}^{\prime}-\psi \pm \sqrt{\frac{2}{p v}\left[E_{T}-U_{e f f}(X)\right]}\right)}{p v \sqrt{\frac{2}{p v}\left[E_{T}-U_{e f f}(X)\right]}}
$$

where the sum is over both signs of the square root. Because of the definition of $E_{c}$, the limits of the $X$ integration do not extend to the ends of the crystal planes, but only to the positions where the potential equals the critical energy. For a straight crystal, these limits are $\pm X_{c}$. For a curved crystal, the potential is asymmetric about the center of the plane. Therefore there is a second value of $X$ such that the potential reaches the critical energy.

$$
E_{c}=U\left(X_{c}\right)-\frac{p v X_{c}}{R}=U\left(X_{\max }\right)+\frac{p v X_{\max }}{R}
$$

as can be seen in Figure 2.3. With this, the acceptance becomes

$$
A=\sum_{ \pm} \int_{-X_{c}}^{X_{\max }} \frac{\mathrm{d} X}{d_{p}} \int_{U_{e f f}(X)}^{E_{c}} \mathrm{~d} E_{T} \frac{\rho\left(x_{p}^{\prime}-\psi \pm \sqrt{\frac{2}{p v}\left[E_{T}-U_{e f f}(X)\right]}\right)}{p v \sqrt{\frac{2}{p v}\left[E_{T}-U_{e f f}(X)\right]}}
$$

For a beam parallel to the crystal, $\rho\left(x^{\prime}\right)=\delta(0)$, and the acceptance is

$$
A=\frac{x_{\max }+x_{c}}{d_{p}}
$$


which in the harmonic approximation becomes

$$
A=\frac{2 x_{c}}{d_{p}}\left(1-\frac{R_{c}}{R}\right) \approx 0.80\left(1-\frac{R_{c}}{R}\right)
$$

In the harmonic approximation, a beam with a uniform angular divergence of $1 / 2 \Phi$, where $\Phi>\theta_{c}$, channeling in a straight crystal will have an acceptance of

$$
A=A_{s}=\frac{2 x_{c}}{d_{p}} \frac{\pi}{4} \frac{\theta_{c, 0}}{\Phi} \approx 0.66 \frac{\theta_{c, 0}}{\Phi}
$$

For a curved crystal the acceptance becomes

$$
A=\frac{2 x_{c}}{d_{p}} \frac{\pi}{4} \frac{\theta_{c, 0}}{\Phi}\left(1-\frac{R_{c}}{R}\right)^{2}=A_{s}\left(1-\frac{R_{c}}{R}\right)^{2}=A_{s} A_{B}
$$

$A_{B}$ is the reduction of the acceptance due to bending. In the harmonic approximation $A_{B}=\left(1-\frac{R_{c}}{R}\right)^{2}$.

\subsubsection{Energy Loss}

If the crystal is misaligned to the beam, the ions do not channel. The approximation that $p_{x}, p_{y} \ll p_{z}$ does not hold, and the ions do not feel the interplanar potential. The ions have a low probability of inelastically scattering and being lost in the crystal. Most particles Coulomb scatter with small angles from the nuclei in the crystal. This is called multiple Coulomb scattering. The distribution of angles is roughly Gaussian. The $95 \%$ spread of angles in the plane for a material of length $z$ is given by

$$
\theta_{0}=\frac{13.6 \mathrm{MeV}}{\beta_{r} c p} Z_{i o n} \sqrt{\frac{z}{X_{0}}}\left[1+0.038 \ln \frac{z}{X_{0}}\right]
$$

where $X_{0}$ is the radiation length of the material [40]. 
In addition to getting an angular kick, particles also lose energy. Energy loss for a relativistic ion passing through a solid is due primarily to ionization and atomic excitation. In an amorphous solid the mean energy loss is given by the Bethe-Bloch formula

$$
\left\langle\frac{\mathrm{d} E}{\mathrm{~d} z}\right\rangle_{\text {random }}=-4 \pi N_{A} r_{e}{ }^{2} m_{e} c^{2} \frac{Z_{\text {ion }}{ }^{2} Z}{A} \frac{1}{\beta_{r}^{2}}\left[\frac{1}{2} \ln \frac{2 m_{e} c^{2} \beta_{r}^{2} \gamma_{r}^{2} T_{\text {max }}}{I^{2}}-\beta_{r}^{2}-\frac{\delta}{2}\right]
$$

where $N_{A}$ is Avogadro's number, $r_{e}$ is the classical electron radius, $m_{e}$ is the electron mass, $Z$ is the atomic number of the absorber, $A$ is the gram molecular weight of the absorber, $T_{\max }$ is the maximum kinetic energy that can be transfered to a free electron, $I \approx Z^{0.9} 16 \mathrm{eV}$ is the mean excitation energy, and $\delta$ is the density correction [40]. The energy loss distribution is given by the Landau distribution [41] which is peaked at an energy below the mean energy loss with a long high energy tail. The peak location is determined by soft collisions with electrons while the long tail is due to hard collisions with electrons and nuclei.

For the case of a channeling particle, the average electron density encountered is lower than in an amorphous solid. This reduces the average energy loss and spread. It has been shown [42] that at high velocities the energy loss for a channeled particle asymptotically approaches

$$
\frac{\mathrm{d} E}{\mathrm{~d} z}(\vec{b}) \approx \frac{1}{2}\left(1+\frac{Z(\vec{b})}{Z}\right)\left\langle\frac{\mathrm{d} E}{\mathrm{~d} z}\right\rangle_{\text {random }}
$$

where $Z(\vec{b})$ is the electron density per atom at impact parameter $\vec{b}$ averaged over a straight line path parallel to the crystal plane. This predicts an energy loss of slightly more than one half of the random case. Equation 2.32 has been shown to agree well with experiments $[42,43]$. Pessimistically assuming that a channeling particle travels 
next to the crystal plane implies

$$
Z(\vec{b}) / Z \approx 2 a_{\mathrm{TF}} / d_{p}
$$

then for a silicon crystal one expects

$$
\frac{\mathrm{d} E}{\mathrm{~d} z}(\vec{b}) \approx 0.6\left\langle\frac{\mathrm{d} E}{\mathrm{~d} z}\right\rangle_{\text {random }}
$$

The fractional energy loss for a $5 \mathrm{~mm}$ silicon crystal and $100 \mathrm{GeV} / \mathrm{u}$ unchanneled gold ion is

$$
\frac{\langle\Delta E\rangle_{\text {random }}}{E}=-1.17 \times 10^{-3}
$$

This is comparable to the nominal energy spread of the beam. For unchanneled protons, the fractional energy loss is approximately 100 times less. For applications at lower energies, energy loss may become important.

\subsubsection{Dechanneling}

There are processes that can cause a particle to dechannel. Among the dominant ones are scattering from a lattice ion, electron or impurity, and bending dechanneling. The goal of this section is not to derive an expression for the probability of dechanneling, but to discuss the processes involved.

In the event of scattering from an electron or nucleus (possibly from an impurity), the change in the transverse energy is given by

$$
\Delta E_{T}=p v \theta \theta_{s}+\frac{1}{2} p v \theta_{s}^{2}
$$

where $\theta_{s}$ is the angular kick from the scattering event [9]. If $\theta_{s}$ is large enough $E_{T}$ 
may become larger than $E_{c}$ and the particle is dechanneled. Detailed treatment of electronic and nuclear scattering is given in several References [9, 44, 45] and will not be discussed here. However, it is useful to define a dechanneling length [9]

$$
L_{D}=\frac{256}{9 \pi^{2}} \frac{p v}{\ln \left(2 m_{e} c^{2} \gamma_{r}^{2} / I\right)-1} \frac{a_{\mathrm{TF}} d_{p}}{Z_{\text {ion }} r_{e} m_{e} c^{2}}
$$

After one dechanneling length $1 / e$ of the particles remain channeled. For protons and fully stripped gold channeling in silicon at RHIC storage energy, $L_{D} \approx 13 \mathrm{~cm}$. For the case of a bent crystal in the harmonic approximation, $L_{D}$ is reduced by a factor $\left(1-R_{c} / R\right)^{2}$. The scattering from impurities can be reduced by using high quality crystals.

Bending dechanneling occurs when a crystal has a position dependent radius of curvature. A change in the radius of curvature changes the local critical energy. Particles with $E_{T} \approx E_{c}$ can find themselves locally with $E_{T}>E_{c}$ and are dechanneled. To reduce this effect, one should bend the crystal as uniformly as possible. The $\mathrm{O}$ shaped design used for RHIC provides a uniform radius of curvature. This is discussed in Section 3.1.1.

\subsubsection{Volume Capture}

The reversibility rule states that the probability of a particle scattering from a channeled state to a dechanneled state is equal to the probability of the reverse process [4]. The mechanisms that cause particle to dechannel also cause particles that are dechanneled to become channeled at some point inside of the crystal volume. This is called volume capture. The following analysis follows Reference [46].

Assuming a bent crystal with radius of curvature $R$, and a beam with a uniform angular divergence $1 / 2 \Phi$, the fraction of particles that are channeled after traversing 
a length $z$ is

$$
f(z)=\frac{2 x_{c}}{d_{p}} \frac{\pi}{4} \frac{\theta_{c}}{\Phi} A_{B} \exp \left[-\frac{z}{L_{D}}\right]
$$

where $L_{D}$ is the bent dechanneling length. The number of particles that are dechanneled per unit length is $-\mathrm{d} f(z) / \mathrm{d} z$ and the angular distribution of these particle downstream of the crystal is given by

$$
\frac{\mathrm{d} f(z)}{\mathrm{d} \theta}=R \frac{\mathrm{d} f(z)}{\mathrm{d} z}=-\frac{R}{L_{D}} f(z)
$$

where the additional angular spread of $\pm \theta_{c}$ is neglected because it is small compared to the bend angle. If the particles enter the crystal from the reverse direction with the input distribution equal to the output distribution of the forward case, then Equation 2.38 gives the probability of particles moving from dechanneled states to channeled states per unit length. This is equal to the capture probability, $w_{s}$, times the transmission factor $\exp \left(-z / L_{D}\right)$. Normalizing Equation 2.38 by the number of particles incident per unit angle gives the capture probability

$$
w_{s}=2 \Phi \frac{\mathrm{d} f(z)}{\mathrm{d} \theta} \exp \left(\frac{z}{L_{D}}\right)=\frac{\pi x_{c}}{d_{p}} \frac{R \theta_{c}}{L_{D}} A_{B}
$$

For most potentials, $A_{B} / L_{D} \approx 1 / L_{D, 0}$ so the capture probability becomes

$$
w_{s}=\frac{\pi x_{c}}{d_{p}} \frac{R \theta_{c}}{L_{D, 0}}
$$

For the case of RHIC, $w_{s} \approx 0.12 \%$. It should be noted that Equation 2.40 overestimates the capture efficiency because it neglects the angular spread of the beam due to channeling. Volume capture is the dominant channeling mode when the incoming ions are not aligned to the crystal planes. 


\subsection{Collimation}

The simplest way to remove particles from the beam halo is to use a movable aperture to scrape away the halo. At low energies this is sufficient. However, particles that impact near and almost parallel to an edge have a high probability of scattering out of the material. At high energies, a collimator may have to be too thick for a significant number of particles to inelastically scatter in it. Halo particles may not have enough of an impact parameter to stay in the collimator while scattering, even though the collimator may be long enough [47]. For these reasons, one collimator is not enough to remove beam halo. Secondary collimators must be placed downstream of a primary collimator to remove particles that escape it.

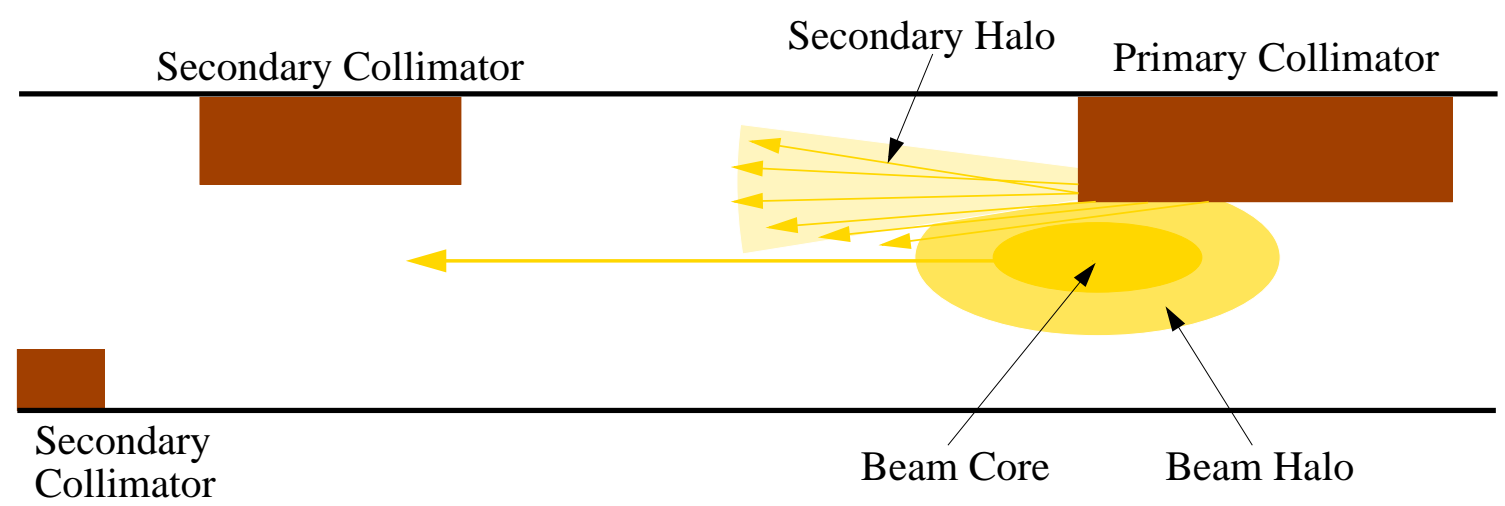

Figure 2.5: A simple collimation system using secondary collimators.

\subsubsection{Two Stage Collimation}

Two stage collimation has been the subject of much study [33, 48, 49]. This section summarizes the results, so that they can be compared to the results of collimating with a crystal. 


\section{One Dimensional Betatron Collimation}

The following section closely follows Reference [33]. Assuming collimation in the horizontal plane only, a particle that just strikes a collimator at position $x_{0}$ has a horizontal action

$$
J_{x}=\frac{x_{0}^{2}}{2 \beta_{x}}
$$

The effects of dispersion are temporarily neglected. If the particle does not scatter inelastically, it will be deflected by an angle $\theta_{x}$ in the collimator jaw. This increases the action of the particle.

$$
J_{x}^{*}=J_{x}+\frac{\beta_{x} \theta_{x}^{2}}{2}
$$

At some location downstream, the particle will be at an extreme position of the phase ellipse.

$$
x_{1}=x_{0} \sqrt{\frac{\beta_{1}}{\beta_{0}}} \cos \Delta \phi+\theta_{x} \sqrt{\beta_{0} \beta_{1}} \sin \Delta \phi
$$

Where the subscripts $\{0,1\}$ denote quantities at the collimator and downstream locations respectively, and $\Delta \phi$ is the phase advance between them. For a particle to hit a secondary collimator at this location it must have scattered by an angle

$$
\theta_{x} \geq \frac{\frac{x_{1}}{\sqrt{\beta_{1}}}-\frac{x_{0}}{\sqrt{\beta_{0}}} \cos \Delta \phi}{\sqrt{\beta_{0}} \sin \Delta \phi}=\frac{x_{n 1}-x_{n 0} \cos \Delta \phi}{\sqrt{\beta_{0}} \sin \Delta \phi} .
$$

The optimum phase advance is the location where the minimum scattering angle is needed to encounter the secondary collimator. This gives the condition

$$
\Delta \phi_{\text {opt }} \approx m \pi \pm \cos ^{-1}\left(\frac{x_{n 0}}{x_{n 1}}\right) \quad m=0, \pm 1, \pm 2, \ldots
$$




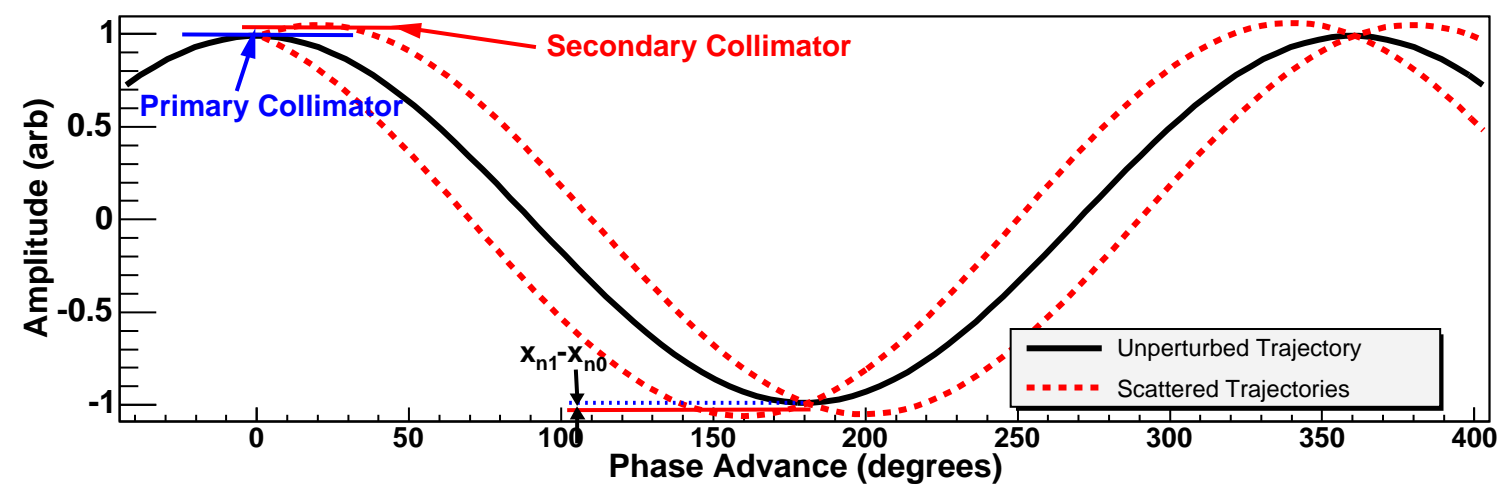

Figure 2.6: The scattered and unscattered particle trajectories as a function of phase advance. The secondary collimators are placed at $30^{\circ}$ and $150^{\circ}$. Adopted from Reference [33].

Computer simulations have shown that an optimum phase advance is [33]

$$
\Delta \phi_{\text {opt }}=m \pi \pm 20^{\circ} \ldots 30^{\circ} .
$$

Choosing the proper phase advance is often a compromise between minimizing the secondary halo and maximizing the distance between the beam and the secondary collimator. For efficient collimation, one should place secondary collimators at $\Delta \phi_{\text {opt }}$ and $\pi-\Delta \phi_{\text {opt }}$ as shown in Figure 2.6.

\section{Two Dimensional Betatron Collimation}

A particle will also receive a kick in the vertical plane. Particles getting large vertical kicks must also be intercepted. A particle that just touches a horizontal collimator has a vertical action of

$$
J_{y}=\frac{1}{2 \beta_{y}}\left[y^{2}+\left(\alpha_{y} y+\beta_{y} y^{\prime}\right)^{2}\right] .
$$


Upon scattering the action becomes

$$
J_{y}^{*}=J_{y}+\frac{\beta_{y} \theta_{y}^{2}}{2}+\theta_{y}\left(\alpha_{y} y+\beta_{y} y^{\prime}\right)
$$

The vertical position of this particle at a secondary collimator is

$$
y_{1}=y_{0} \sqrt{\frac{\beta_{y 1}}{\beta_{y 0}}}\left(\cos \Delta \phi_{y}+\alpha_{y 0} \sin \Delta \phi_{y}\right)+\left(y_{0}^{\prime}+\theta_{y}\right) \sqrt{\beta_{y 0} \beta_{y 1}} \sin \Delta \phi_{y} .
$$

The phase advance in the vertical plane is generally not the same as in the horizontal. If there is a vertical collimator inserted to $y_{1}$ at this phase advance the particle will hit it if $y \geq y_{1}$. This gives a minimum kick of

$$
\theta_{y, \text { min }}=-y_{0}^{\prime}+\frac{\frac{y_{1}}{\sqrt{\beta_{y 1}}}-\frac{y_{0}}{\sqrt{\beta_{y 0}}}\left(\cos \Delta \phi_{y}+\alpha_{y 0} \sin \Delta \phi_{y}\right)}{\sqrt{\beta_{y 0}} \sin \Delta \phi_{y}}=-y_{n 0}^{\prime}+\frac{y_{n 1}-y_{n 0} \cos \Delta \phi_{y}}{\sqrt{\beta_{y 0}} \sin \Delta \phi_{y}}
$$

to strike a vertical secondary collimator. Because of the random nature of the incident vertical position and angle of the particle on the horizontal collimator, it is difficult to make general statements about a $2 \mathrm{D}$ collimation system. Some special cases are discussed in Reference [49].

\section{Effects of Dispersion}

In addition to the scattering in the collimator jaw, it is also important to account for the energy loss. Energy loss will affect the particle orbit and may affect the optimal location of secondary collimators. Synchrotron motion can be neglected because the

synchrotron tune is much smaller than the betatron tune, and particles near the bucket edge have small synchrotron tunes compared to particles near the center of the RF bucket. The remainder of this section follows Reference [49].

The removal of particles near or beyond the edge of the RF bucket can be 
accomplished by placing a horizontal collimator at a location with a large normalized dispersion. The collimator should not scrape particles far inside the RF bucket. So a minimum momentum offset, $\delta_{\text {cut }}$, is chosen such that particles with $\delta<\delta_{\text {cut }}$ will not touch the collimator if it is inside the transverse dynamic aperture. A particle with an amplitude that is near the transverse dynamic aperture, will have a maximum normalized position, $x_{n, \max }$. It just touches the collimator if it satisfies

$$
x_{0}=x_{n, \max } \sqrt{\beta}+D \delta_{c u t} .
$$

Particles with smaller $x_{n}$ or $\delta$ will not touch the collimator. Particles with larger $x_{n}$ are beyond the dynamic aperture and it is desirable for those particles to hit the collimator.

The maximum momentum offset that will pass the collimator is for particles with no betatron amplitude

$$
x_{0}=D \delta_{\max }
$$

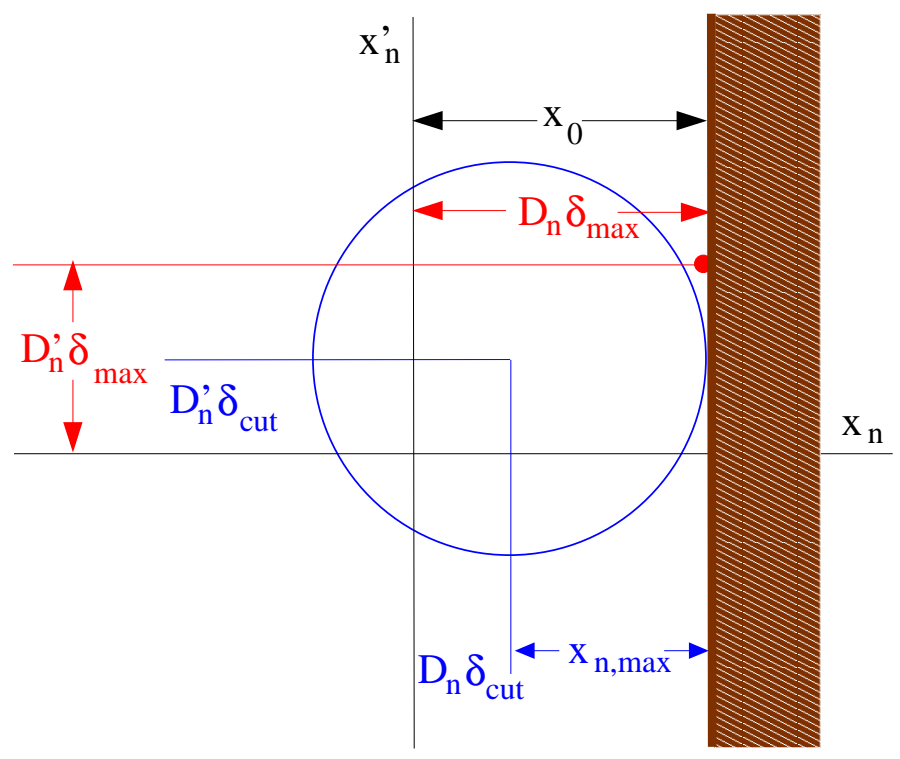

Figure 2.7: Normalized phase space used for momentum collimation 
The maximum momentum offset is then

$$
\delta_{\max }=\frac{x_{n, \max } \sqrt{\beta}}{D}+\delta_{c u t}=\frac{x_{n, \max }}{D_{n}}+\delta_{c u t} .
$$

This equation shows that $\delta_{\max }$ is always greater than $\delta_{c u t}$. This is because particles with smaller betatron amplitudes can pass the collimator even though they have a momentum offsets larger than $\delta_{c u t}$. The collimator location should be such that $\delta_{\max }$ is tolerable for the machine running conditions. The maximum momentum offset can be reduced if the collimator is placed at a large $D_{n}$ location.

To analyze the effects of dispersion on the collimation system, assume that a particle with momentum offset $\delta_{i n}$ touches the collimator at transverse location $x_{0}$ at the out most location of its phase ellipse. From Equation 1.31 the invariant of the motion is

$$
A=\frac{\left(x_{0}-D \delta_{i n}\right)^{2}+\left(D \alpha+D^{\prime} \beta\right)^{2} \delta_{i n}^{2}}{2 \beta}
$$

After scattering in the collimator, the particle receives an angular kick $\theta_{x}$ and a momentum reduction $-\Delta \delta$. The invariant becomes

$$
\begin{aligned}
A^{*}= & A+\frac{\beta \theta_{x}^{2}}{2}+\Delta \delta\left[\frac{x_{0} D}{\beta}+\theta_{x}\left(D \alpha+D^{\prime} \beta\right)\right] \\
& +\frac{\Delta \delta^{2}}{2}\langle\mathcal{H}\rangle-\delta_{\text {in }}\left[\Delta \delta\langle\mathcal{H}\rangle+\theta_{x}\left(D \alpha+D^{\prime} \beta\right)\right] .
\end{aligned}
$$

For $\Delta \delta=0$ the above equation is the same as Equation 2.42 with an added term for the initial momentum offset of the particle.

Downstream of the collimator the position of the particle is

$$
x_{1}=\left(x_{0}-D_{0} \delta_{i n}\right) \sqrt{\frac{\beta_{1}}{\beta_{0}}}\left(\cos \Delta \phi+\alpha_{0} \sin \Delta \phi\right)+\left(\theta_{x}-D_{0}^{\prime} \delta_{i n}\right) \sqrt{\beta_{0} \beta_{1}} \sin \Delta \phi+D_{1}\left(\delta_{i n}-\Delta \delta\right) .
$$


For a particle to hit a secondary collimator at this location it must have scattered by an angle

$$
\begin{aligned}
\theta_{x} & \geq \frac{\frac{x_{1}}{\sqrt{\beta_{1}}}-\frac{x_{0}}{\sqrt{\beta_{0}}} \cos \Delta \phi}{\sqrt{\beta_{0}} \sin \Delta \phi}+\left(\delta_{i n}-\Delta \delta\right) \frac{\frac{D_{0}}{\sqrt{\beta_{0}}} \cos \Delta \phi-\frac{D_{1}}{\sqrt{\beta_{1}}}}{\sqrt{\beta_{0}} \sin \Delta \phi}-\Delta \delta\left(D_{0}^{\prime}+\frac{\alpha_{0}}{\beta_{0}} D_{0}\right) \\
& =\frac{x_{n 1}-x_{n 0} \cos \Delta \phi}{\sqrt{\beta_{0}} \sin \Delta \phi}+\left(\delta_{i n}-\Delta \delta\right) \frac{D_{n 0} \cos \Delta \phi-D_{n 1}}{\sqrt{\beta}_{0} \sin \Delta \phi}-\frac{\Delta \delta D_{n 0}^{\prime}}{\sqrt{\beta_{0}}}
\end{aligned}
$$

The equation for the dispersion is given by Equation 1.25b. The solution can be written in the form

$$
\begin{aligned}
D_{1}= & D_{0} \sqrt{\frac{\beta_{1}}{\beta_{0}}}\left(\cos \Delta \phi+\alpha_{0} \sin \Delta \phi\right)+D_{0}^{\prime} \sqrt{\beta_{1} \beta_{0}} \sin \Delta \phi \\
& -a_{1}\left(s_{1}\right) \sqrt{\beta_{1}} \cos \Delta \phi+a_{2}\left(s_{1}\right) \sqrt{\beta_{1}} \sin \Delta \phi
\end{aligned}
$$

where

$$
\begin{aligned}
& a_{1}\left(s_{1}\right)=\int_{s_{0}}^{s_{1}} \frac{\sqrt{\beta\left(s^{\prime}\right)}}{\rho\left(s^{\prime}\right)} \sin \phi\left(s^{\prime}\right) \mathrm{d} s^{\prime} \\
& a_{2}\left(s_{1}\right)=\int_{s_{0}}^{s_{1}} \frac{\sqrt{\beta\left(s^{\prime}\right)}}{\rho\left(s^{\prime}\right)} \cos \phi\left(s^{\prime}\right) \mathrm{d} s^{\prime}
\end{aligned}
$$

which includes the homogeneous solution.

This can be inserted into the above Equation 2.56 to obtain

$$
\theta_{x} \geq x_{n 1}-\frac{x_{n 0} \cos \Delta \phi}{\sqrt{\beta}_{0} \sin \Delta \phi}+\left(\delta_{i n}-\Delta \delta\right) \frac{a_{1}\left(s_{1}\right) \cot \Delta \phi-a_{2}\left(s_{1}\right)}{\sqrt{\beta}_{0}}-\delta_{i n} \frac{D_{n 0}^{\prime}}{\sqrt{\beta}_{0}} .
$$

It is difficult to make any general statements about the performance of a momentum collimation system, because $a_{1}(s)$ and $a_{2}(s)$ depend on the accumulated phase in a nontrivial way. However, for the special case of a straight section $a_{1}(s)$ 
and $a_{2}(s)$ are zero. The angles that hit the collimator become

$$
\theta_{x} \geq \frac{x_{n 1}-x_{n 0} \cos \Delta \phi}{\sqrt{\beta}_{0} \sin \Delta \phi}-\delta_{i n} \frac{D_{n 0}^{\prime}}{\sqrt{\beta_{0}}}
$$

In a drift $\theta_{x}$ is independent of energy loss. This can be disadvantageous for particles that lose a lot of energy in the primary collimator but do not gain a large enough angular kick. These particles will not get intercepted by the secondary collimator. It is also important to note from the above equation is that if $D_{n 0}^{\prime}=0$ the efficiency of the collimation system is the same for all energies. This has the advantage that the secondary collimator can be placed at a location that is convenient for both momentum and betatron collimation.

\subsubsection{Two Stage Crystal Collimation}

The main advantage of crystal collimation is that the angular kick received at the crystal is known for particles that channel. This allows optimal placement of the secondary collimator so that there is a large impact parameter and a larger retraction from the beam than can be achieved than with a standard collimation system.

The angular kick that a channeled particle will receive from a bent crystal is

$$
\theta_{x}=\theta_{b}+\theta_{r} \approx \theta_{b}
$$

where $\left|\theta_{r}\right| \leq \theta_{c}$ is the angle of the particle with respect to the crystal planes when it exits the crystal.

With this small angular spread, a secondary collimator can be placed at a location where $\left|x_{n 1}\right|>\left|x_{n 0}\right|$. The phase advance to hit a secondary collimator at position 
$x_{1}$ is

$$
\begin{aligned}
\cos \Delta \phi & =\frac{\frac{x_{1}}{\sqrt{\beta_{1}}} \frac{x_{0}}{\sqrt{\beta_{0}}} \pm \sqrt{\beta_{0}} \theta_{x} \sqrt{\frac{x_{0}^{2}}{\beta_{0}}+\beta_{0} \theta_{x}^{2}-\frac{x_{1}{ }^{2}}{\beta_{1}}}}{\frac{x_{0}^{2}}{\beta_{0}}+\beta_{0} \theta_{x}^{2}} \\
& =\frac{x_{n 0} x_{n 1} \pm \theta_{x n} \sqrt{x_{n 0}{ }^{2}+\theta_{x n}^{2}-x_{n 1}^{2}}}{x_{n 0}{ }^{2}+\theta_{x n}^{2}}
\end{aligned}
$$

where $\theta_{x n}$ is the normalized angular kick to the channeled beam. The expression under the square root is negative when $x_{n 1}{ }^{2}>x_{n 0}{ }^{2}+\theta_{x n}{ }^{2}$. This occurs because the maximum normalized separation of the channeled beam and the crystal shadow, $x_{n 1}-x_{n 0}$, is achieved when $x_{n 1}{ }^{2}=x_{n 0}{ }^{2}+\theta_{x n}{ }^{2}$. Inserting this into Equation 2.62 gives the optimal phase advance of

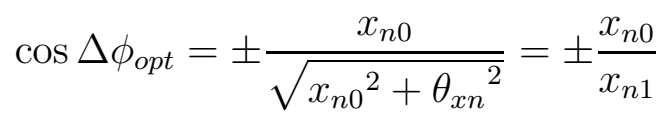

which is identical to Equation 2.45, with the exception that in the above equation $x_{n 1}$ is given in terms of the crystal bend angle, and is not chosen to intercept an angular range of scattered particles. A secondary collimator placed at this phase advance will be able to intercept the channeled particles at their extreme betatron oscillation. This will minimize the effects of halo from the secondary collimator touching the beam core.

To include the effects of dispersion, it is useful to start with Equation 2.55. As in the non-dispersive case, the angle imparted by the crystal is approximately the bend angle, $\theta_{x} \approx \theta_{b}$. Using Equations $2.58 \mathrm{a}$ and $2.58 \mathrm{~b}$ it is possible to rewrite Equation 
2.55 as

$$
\begin{aligned}
x_{1}= & {\left[x_{0}-D_{0} \Delta \delta-a_{1}\left(s_{1}\right) \sqrt{\beta_{0}}\left(\delta_{i n}-\Delta \delta\right)\right] \sqrt{\frac{\beta_{1}}{\beta_{0}}} \cos \Delta \phi } \\
& +\left[\theta_{x}-D_{0}^{\prime} \Delta \delta+\frac{\alpha_{0}}{\beta_{0}}\left(x_{0}-D_{0} \Delta \delta\right)+\frac{a_{2}\left(s_{1}\right)}{\sqrt{\beta_{0}}}\left(\delta_{i n}-\Delta \delta\right)\right] \sqrt{\beta_{0} \beta_{1}} \sin \Delta \phi
\end{aligned}
$$

From this, one can solve for the phase advance

$$
\cos \Delta \phi=\frac{\frac{X}{\sqrt{\beta_{0}}} \frac{x_{1}}{\sqrt{\beta_{1}}} \pm \sqrt{\beta_{0}} X^{\prime} \sqrt{\frac{X^{2}}{\beta_{0}}+\beta_{0} X^{\prime 2}-\frac{x_{1}^{2}}{\beta_{1}}}}{\frac{X^{2}}{\beta_{0}}+\beta_{0} X^{\prime 2}}
$$

where $X$ and $X^{\prime}$ are the first and second bracketed terms in Equation 2.64 respectively. Because $a_{1}\left(s_{1}\right)$ and $a_{2}\left(s_{1}\right)$ depend on the accumulated phase advance in a nontrivial way and $\Delta \delta$ is unknown, it is impossible to make a general statement about the optimal phase advance. However for thin crystals and, $\Delta \delta \ll \delta_{\text {in }}$ and can be neglected, which is not the case for long collimators. In the case of a drift, $a_{1}=a_{2}=0$ and Equation 2.65 is identical to 2.63.

If the channeling efficiency of the crystal is high and the angular spread of the particles that hit the crystal is small with respect to the crystal angular acceptance, the number of particles that scatter in the crystal will be low. However, if this is not the case, the number of scattered particles can be large. These particles will occupy all angles between $x_{p}^{\prime}$ and $\theta_{x}$ after the crystal negating any advantage of using a crystal. Because of this, they may miss the secondary collimator, unless $x_{n 1}=x_{n 0}$. Therefore it is important to place the crystal collimator at a location with a small angular spread.

The angular spread of the beam that encounters the crystal can be calculated 

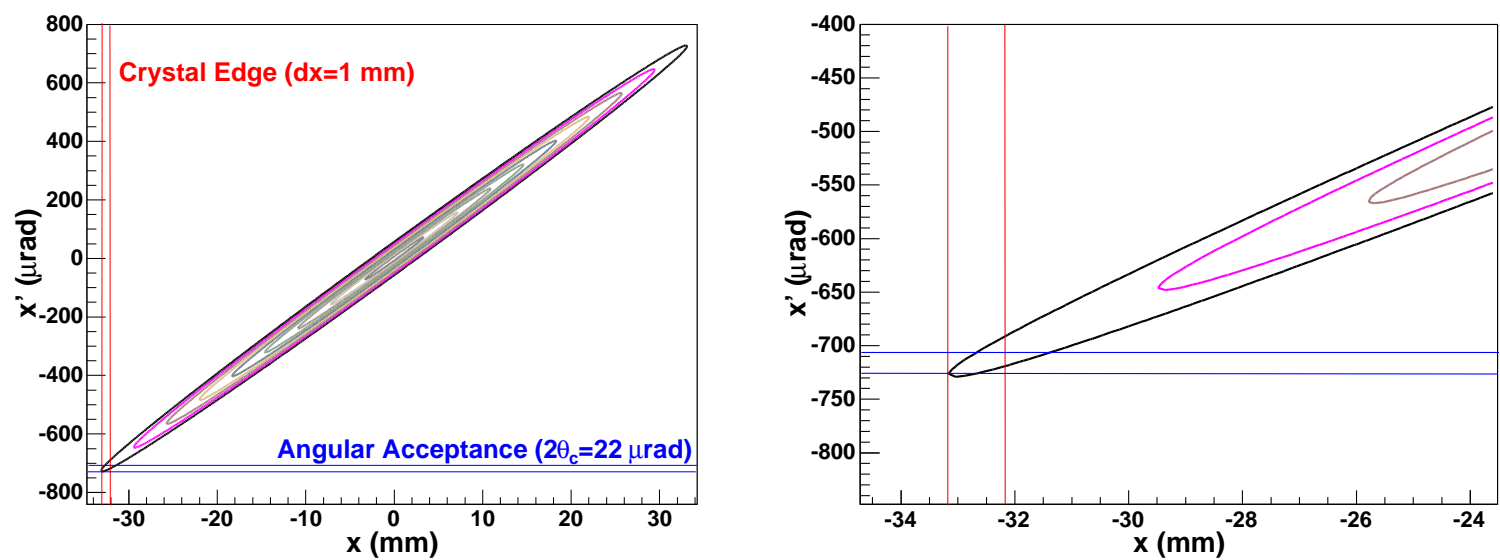

Figure 2.8: Beam Horizontal Phase Space at the RHIC Crystal Collimator for $\beta^{*}=2 m$ at PHENIX interaction region. Each contour is $1 \sigma$ of the beam size from emittance. The right picture magnifies the area of the crystal acceptance.

from the beam parameters. The beam distribution is assumed to be

$$
\rho(A, \delta)=\frac{1}{\sqrt{2 \pi \sigma_{p}} \epsilon} \exp \left[-\frac{A}{\epsilon}\right] \exp \left[-\frac{\delta^{2}}{2 \sigma_{p}^{2}}\right]
$$

where $A$ is the invariant of the motion defined in Equation 1.31, $\epsilon$ is the RMS unnormalized beam emittance and $\sigma_{p}$ is the RMS momentum spread. Because $A=A\left(x, x^{\prime}, \delta\right)$ and $\rho(A, \delta)=\rho\left(x, x^{\prime}, \delta\right)$, all particle momenta are integrated over to obtain $\rho\left(x, x^{\prime}\right)$. The particle distribution becomes

$$
\rho\left(x, x^{\prime}\right)=\frac{\exp \left[-\frac{x^{\prime 2}\left(\beta \epsilon+D^{2} \sigma_{p}^{2}\right)-2 x x^{\prime}\left(-\alpha \epsilon+D D^{\prime} \sigma_{p}{ }^{2}\right)+x^{2}\left(\gamma \epsilon+D^{\prime 2} \sigma_{p}{ }^{2}\right)}{2 \epsilon \sigma_{p}^{2}\left[\gamma D^{2}+2 \alpha D D^{\prime}+\beta D^{\prime 2}+\epsilon / \sigma_{p}^{2}\right]}\right]}{2 \pi \sigma_{p} \sqrt{\epsilon\left(\gamma D^{2}+2 \alpha D D^{\prime}+\beta D^{\prime 2}+\epsilon / \sigma_{p}^{2}\right)}} .
$$

The contours of this distribution are drawn in Figure 2.8 at the location of the crystal collimator. It is convenient to calculate the standard deviations of this distribution [50]

$$
\sigma_{x}^{2}=\epsilon \beta+D^{2} \sigma_{p}^{2}
$$




$$
\begin{gathered}
{\sigma_{x^{\prime}}}^{2}=\gamma \epsilon+D^{\prime 2}{\sigma_{p}}^{2} \\
\sigma_{x x^{\prime}}=-\alpha \epsilon+D D^{\prime}{\sigma_{p}}^{2}
\end{gathered}
$$

which are mean square beam size, mean square divergence and correlation at the location of the crystal collimator respectively.

The particle angular distribution on the crystal face is given by the conditional probability distribution [50] for a particle to have a position $x_{0} \leq x \leq x_{0}+\Delta x$ where $\Delta x$ is the width of the crystal face

$$
\rho\left(x^{\prime} \mid x_{0}\right)=\frac{\int_{x_{0}}^{x_{0}+\Delta x} \rho\left(x, x^{\prime}\right) \mathrm{d} x}{\int_{x_{0}}^{x_{0}+\Delta x} \mathrm{~d} x \int_{-\infty}^{\infty} \rho\left(x, x^{\prime}\right) \mathrm{d} x^{\prime}} .
$$

Using this conditional probability distribution, the average angle and angular spread can be calculated. The average angle of particles hitting the crystal is

$$
x_{p}^{\prime}=\sqrt{\frac{2}{\pi}} \frac{\sigma_{x x^{\prime}}}{\sigma_{x}} \frac{\left[1-\exp \left(\frac{\Delta x\left(2 x_{0}+\Delta x\right)}{2 \sigma_{x}^{2}}\right)\right] \exp \left(-\frac{\left(x_{0}+\Delta x\right)^{2}}{2 \sigma_{x}^{2}}\right)}{\operatorname{Erf}\left[\frac{x_{0}}{\sqrt{2} \sigma_{x}}\right]-\operatorname{Erf}\left[\frac{x_{0}+\Delta x}{\sqrt{2} \sigma_{x}}\right]} .
$$

The divergence is given by the width of the distribution 


$$
\begin{aligned}
& \sigma_{x^{\prime}}\left(x_{0}\right)=\exp \left[-\frac{\left(x_{0}+\Delta x\right)^{2}}{2 \sigma_{x}^{2}}\right]\left\{\frac{-2}{\pi} \frac{\sigma_{x x^{\prime}}{ }^{2}}{\sigma_{x}^{2}} \frac{\left[1-\exp \left(\frac{\Delta x\left(2 x_{0}+\Delta x\right)}{2 \sigma_{x}^{2}}\right)\right]^{2}}{\left[\operatorname{Erf}\left(\frac{x_{0}}{\sqrt{2} \sigma_{x}}\right)-\operatorname{Erf}\left(\frac{x_{0}+\Delta x}{\sqrt{2} \sigma_{x}}\right)\right]^{2}}\right. \\
& +\left[\sigma_{x^{\prime}}^{2} \exp \left[\frac{\left(x_{0}+\Delta x\right)^{2}}{2 \sigma_{x}^{2}}\right]+\sqrt{\frac{2}{\pi}} \frac{\sigma_{x x^{\prime}}^{2}}{\sigma_{x}^{2}} \frac{\Delta x+x_{0}\left[1-\exp \left(\frac{\Delta x\left(2 x_{0}+\Delta x\right)}{2 \sigma_{x}{ }^{2}}\right)\right]}{\sigma_{x}\left[\operatorname{Erf}\left(\frac{x_{0}}{\sqrt{2} \sigma_{x}}\right)-\operatorname{Erf}\left(\frac{x_{0}+\Delta x}{\sqrt{2} \sigma_{x}}\right)\right]}\right] \\
& \left.\quad \exp \left[\frac{\left(x_{0}+\Delta x\right)^{2}}{2 \sigma_{x}^{2}}\right]\right\}^{\frac{1}{2}}
\end{aligned}
$$

Because collimators generally operate such that $x_{0} \geq 6 \sigma_{x}$, and $\Delta x<\sigma_{x}$ the difference of the error functions approaches zero, and the exponential terms approach one. So it is convenient to expand Equations 2.70 and 2.71 for small $\Delta x$.

To lowest order in $\Delta x$ the average angle hitting the crystal is

$$
x_{p}^{\prime}=\left(x_{0}+\frac{\Delta x}{2}\right) \frac{\sigma_{x x^{\prime}}}{\sigma_{x}^{2}} .
$$

Higher order terms quickly approach zero. In the case of small momentum spread or small normalized dispersion, as in the case of the RHIC collimator location, this is reduced further to

$$
x_{p}^{\prime} \approx\left(x_{0}+\frac{\Delta x}{2}\right) \frac{-\alpha}{\beta}
$$

which can be obtained from single particle dynamics for a particle that hits in the middle of the crystal face. If the angle of the crystal planes with respect to the closed orbit is $x_{p}^{\prime}$ then the crystal is said to be properly aligned to the beam. For $\alpha \neq 0$, the crystal alignment depends on the distance between the beam center and the crystal edge. 
Table 2.3: Design horizontal beam parameters at crystal for various $\beta_{P H E N I X}^{*}$

\begin{tabular}{|c|c|c|c|c|c|c|c|}
\hline$\beta_{P H E N I X}^{*}[\mathrm{~m}]$ & $\beta[\mathrm{m}]$ & $\alpha$ & $D[\mathrm{~m}]$ & $D^{\prime}$ & $\sigma_{x}[\mathrm{~mm}]$ & $\sigma_{x^{\prime}}[\mu \mathrm{rad}]$ & $\begin{array}{c}\sigma_{x x^{\prime}} \\
{[\mathrm{mm}-\mathrm{mrad}]}\end{array}$ \\
\hline 1 & 1155 & -26.5 & -0.864 & -0.0162 & 5.31 & 121 & 0.642 \\
2 & 581 & -12.8 & -0.152 & 0.0002 & 3.70 & 81.1 & 0.298 \\
3 & 391 & -8.33 & -0.013 & 0.0032 & 3.02 & 65.0 & 0.195 \\
5 & 242 & -4.95 & 0.103 & 0.0055 & 2.39 & 50.1 & 0.117 \\
10 & 129 & -2.67 & 0.117 & 0.0056 & 1.75 & 39.0 & 0.064 \\
\hline
\end{tabular}

To fifth order in $\Delta x$, the angular width becomes

$$
\begin{aligned}
\sigma_{x^{\prime}}\left(x_{0}\right)= & \frac{\sqrt{\epsilon\left(\epsilon+\langle\mathcal{H}\rangle \sigma_{p}^{2}\right)}}{\sigma_{x}}+\frac{\sigma_{x x^{\prime}}^{2} \Delta x^{2}}{24 \sigma_{x}^{3} \sqrt{\epsilon\left(\epsilon+\langle\mathcal{H}\rangle \sigma_{p}^{2}\right)}} \\
& \left\{1-\frac{\Delta x^{2}}{240 \sigma_{x}^{2}}\left[8+5 \frac{-\alpha \epsilon \sigma_{x x^{\prime}}+D^{2} D^{\prime 2} \sigma_{p}{ }^{4}}{\epsilon\left(\epsilon+\langle\mathcal{H}\rangle \sigma_{p}{ }^{2}\right)}+12 \frac{x_{0}{ }^{2}}{\sigma_{x}^{2}}\right]-\frac{x_{0} \Delta x^{3}}{20 \sigma_{x}{ }^{4}}\right\}+O\left(\Delta x^{6}\right)
\end{aligned}
$$

From Equations 2.71 and 2.74 and Figure 2.8 it can be seen that the optimal location for a crystal collimator is at a location where $\alpha=D^{\prime}=0$. At these locations the phase ellipse is flat and the beam divergence on the crystal is minimized. It can also be seen that a local maximum in the $\beta$ function is also desirable to further reduce the beam divergence. Both of these minimize the angular spread of the beam that encounters the crystal for all $x_{0}$ and therefore increases the channeling efficiency. In addition, the angle and angular spread of the incident beam on the crystal is independent of $x_{0}$ which greatly simplifies the operation of a crystal collimator. Otherwise the crystal collimator needs to be realigned whenever its position is changed. Unfortunately, in RHIC, there are no warm spaces where $\left\{\alpha, D^{\prime}\right\} \approx 0$ and $\beta$ is a maximum. Because $\sigma_{x^{\prime}}\left(x_{0}\right)$ depends strongly on $\sigma_{x x^{\prime}}$ and thus $\alpha$, errors in the $\beta$ function can have a large effect on this width. This can have a devastating effect on the channeling efficiency as can be seen from Equation 2.29.

Figure 2.9 shows a plot of $\sigma_{x^{\prime}}\left(x_{0}\right)$ vs. $\alpha$ and $D^{\prime}$ for the model RHIC lattice for 


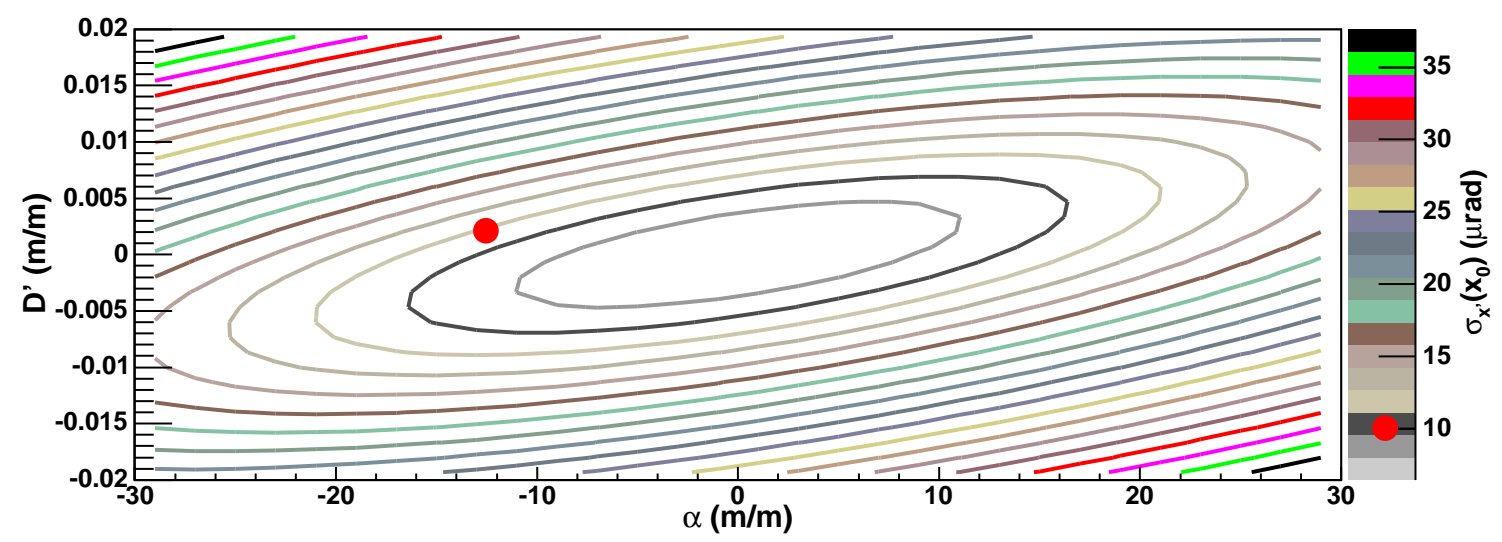

Figure 2.9: Width of beam distribution on crystal vs. $\alpha$ and $D^{\prime}$ for $\beta^{*}=2 \mathrm{~m}$ at the PHENIX interaction region. The marker shows the model $\alpha$ and $D^{\prime}$.

$\beta^{*}=2 \mathrm{~m}$ at the PHENIX interaction region. For this lattice, $\sigma_{x^{\prime}}\left(x_{0}\right)=9.98 \mu \mathrm{rad}$ and the estimated channeling efficiency is $71 \%$.

This model of the beam distribution does not include the scattered particles from the crystal or emittance growth effects explicitly. However, there is the assumption that there are always particles impacting the entire width of the crystal face. This is most important for calculating $\sigma_{x^{\prime}}\left(x_{0}\right)$. Since the crystal collimator is operated in the tails of the beam distribution, $\sigma_{x^{\prime}}\left(x_{0}\right)$ is particularly sensitive to the beam distribution in the tails. Therefore, deviations from a Gaussian shape are important in these limits. Measurements with the scraper show that the halo in RHIC deviates from a gaussian shape. This is discussed in Section 6.2.

The greatest advantage of a bent crystal collimator is the small angular spread and well defined angle of the ions that exit the crystal. This makes intercepting these particles downstream easier than the case of a conventional two stage collimation system. However, it is necessary to match the beam shape to the crystal by reducing the angular spread of the beam that hits the crystal. This places limitations on the possible locations for a crystal collimator, and strict tolerances on optical errors. These limitations are not important for a traditional two stage collimation system. 


\section{Chapter 3}

\section{The RHIC Collimation System}

The RHIC collimation system prior to the FY2004 run consists of a single copper scraper in each ring and a bent crystal collimator in the yellow ring. The scrapers are located downstream of the PHENIX detector in the drift space between the outgoing triplet and the spin rotator magnet in each ring. The yellow scraper is located in the 7 o'clock side of PHENIX and the blue scraper is located on the 8 o'clock side of PHENIX. The blue and yellow scraper are placed $47.2 \mathrm{~m}$ and $46.75 \mathrm{~m}$ downstream of the PHENIX detector respectively. The bent crystal collimator, installed before the FY2001 run, is $7.8 \mathrm{~m}$ upstream of the yellow scraper. Figure 3.1 shows the configuration of the system. This section of RHIC was chosen as the collimator location because it is a warm drift space with no other instrumentation. Placing collimators at other locations would mean costly cryogenic bypasses or interference with other accelerator components.

The collimation system is upgraded for the FY2004 run. This upgrade is discussed in Chapter 7. This chapter discusses the instrumentation of the RHIC collimation system before the upgrade, along with the initial experience in commissioning the copper scrapers. 


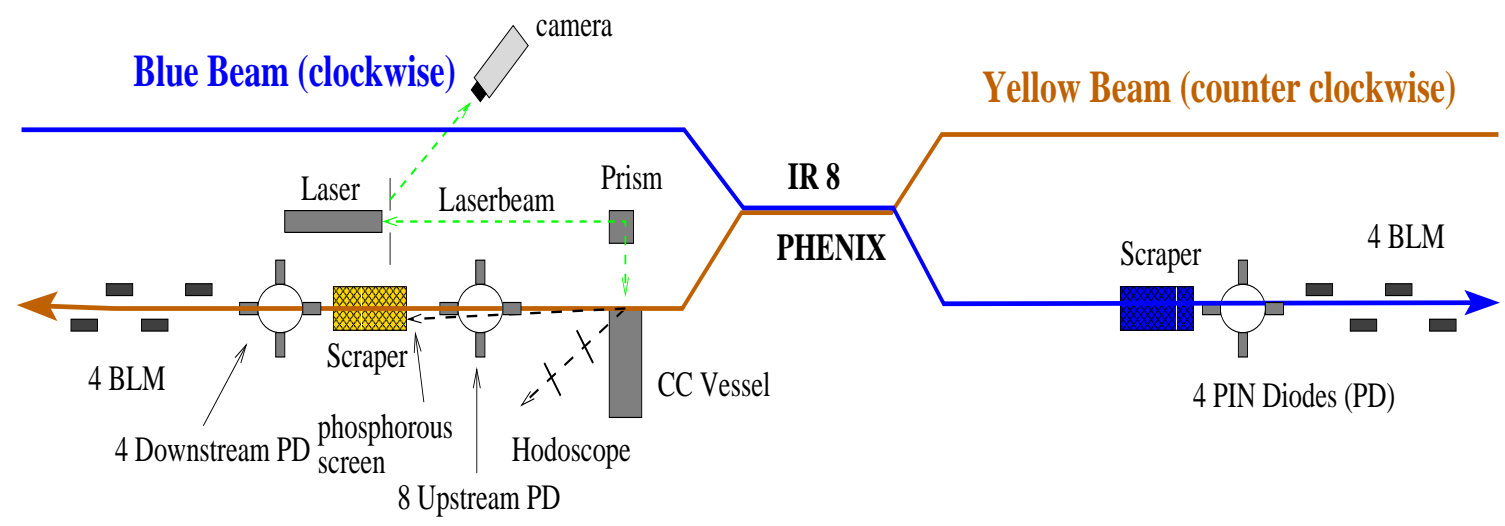

Figure 3.1: RHIC Collimation System. The center of RHIC is toward the bottom of the figure.

\subsection{Hardware}

Figure 3.1 shows a schematic of the RHIC collimation system. Each ring contains:

- A copper scraper.

- An array of four "downstream" PIN diodes downstream of the scrapers.

- Four dedicated ionization chamber Beam Loss Monitors (BLMs) [51] to measure large beam losses downstream of the scraper.

- A dual plane Beam Position Monitor (BPM) on each end of the collimation section to measure the beam position and angle at the scrapers.

The yellow ring contains additional hardware:

- the crystal collimator.

- eight "upstream" PIN diodes between the crystal collimator and the scraper, used to detect particles scattered from the crystal.

- two scintillators that form a hodoscope used the monitor particles scattered at large angles from the crystal. 
- A laser and charge coupled device (CCD) camera to measure the angle of the crystal relative to its line of motion.

- A camera to image the phosphor screen on the yellow scraper (not shown).

\subsubsection{Crystal}

The crystal that is used in RHIC is an O-shaped silicon crystal, produced in PNPI, St. Petersburg, Russia, using the (110) planes for channeling. It is shown in Figure 3.2. The length of the side that channels the beam is $5 \mathrm{~mm}$. The bend angle of the crystal is $\theta_{b} \approx 440 \mu \mathrm{rad}$. This bend is achieved with the stainless steel holder by applying inward pressure on the long sides of the crystal, forcing the short side to bulge outward. The beam enters the crystal from the top of Figure 3.2, and the channeled beam is deflected to the right.

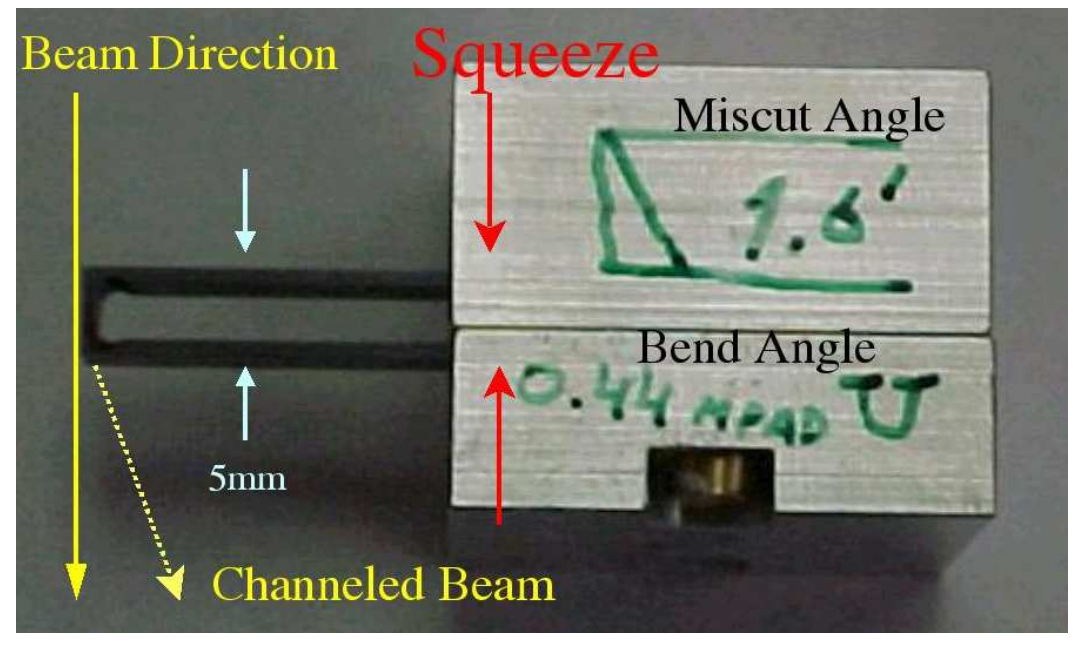

Figure 3.2: The RHIC Crystal.

The crystal was manufactured with a miscut angle of $\theta_{\text {miscut }}=465 \mu \mathrm{rad}$. The miscut angle is the angle between the normal to the beam input face and the crystal planes, as shown in Figure 3.3. In manufacturing a crystal, there will always be some miscut angle. If the miscut angle is negative, the particles will be channeled 
toward the side of the crystal, and may not traverse the whole length of the crystal. These particles will not receive the full deflection. To ensure that this does not occur accidentally, a positive miscut is always manufactured into the crystal.

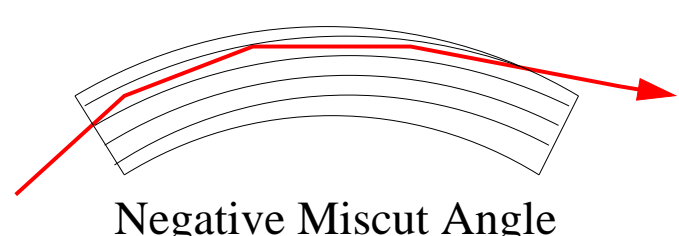

Negative Miscut Angle

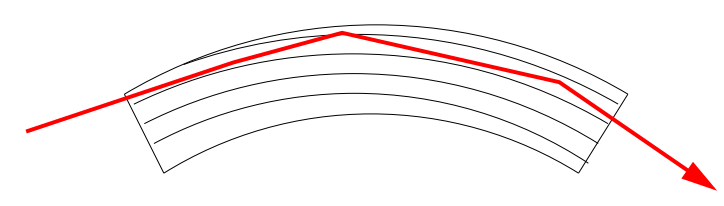

Positive Miscut Angle

Figure 3.3: Effect of the miscut angle, $\theta_{\text {miscut }}$, on channeled particles

\subsubsection{Crystal Vessel}

Figure 3.4 shows the vessel used to house the crystal collimator. The crystal sits on the end of a lever arm inside the vessel near the opening to the beamline, as shown in Figure 3.5. The lever arm pivots on a beam mounted in the orange five way cross. A piezoelectric inchworm mounted in the orange cross pushes against the lever arm to adjust the angle of the crystal in the beamline. A Linear Voltage Differential Transformer (LVDT) mounted in the other half of the cross measures the movement of the inchworm. Limit switches inside of the vessel prevent the lever arm from traveling too far. These limit the rotation to $\pm 7 \mathrm{mrad}$ relative to the axis of motion.

The five way cross is mounted on a translation stage driven by a stepper motor to move perpendicularly to the beamline, to insert and retract the crystal collimator. An LVDT attached to the stage is used to measure the movement of the stage. Limit switches mounted on the crystal collimator stand limit the travel of the stage to ensure a maximum crystal insertion of $12.5 \mathrm{~mm}$ from the center of the beam pipe. A magnetic brake attached to the stage is used to lock the stage in place.

The voltage from the LVDT attached to the stage drifted and showed hysteresis 


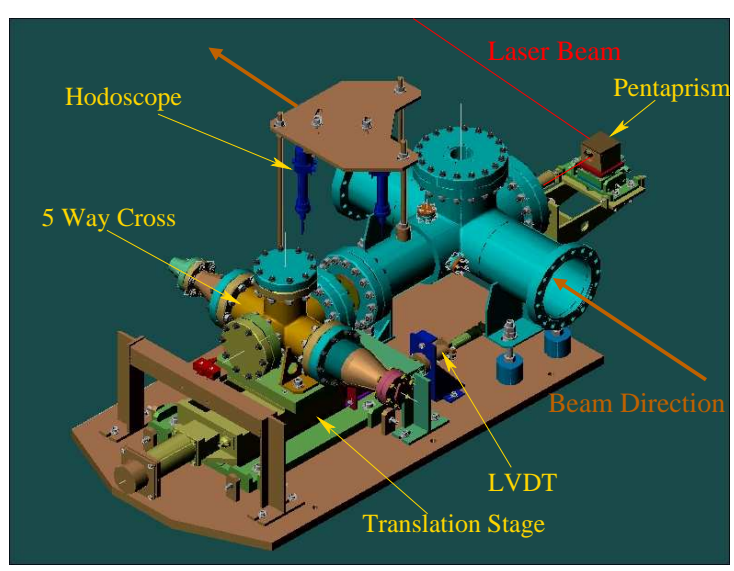

Figure 3.4: Vacuum vessel used to house the crystal collimator apparatus.

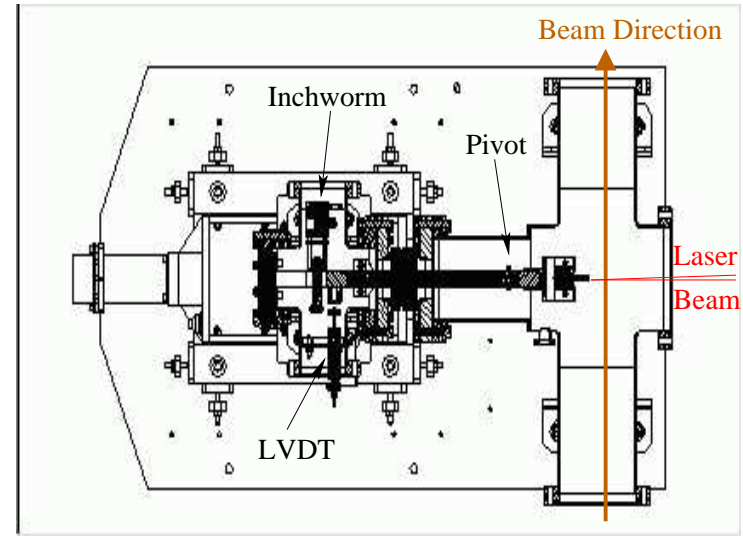

Figure 3.5: Section view of the crystal vessel showing the angular positioning system.

with temperature changes. Therefore the LVDT did not allow accurate measurement of the crystal position. We decided that the best measure of the crystal horizontal position was to count the number of stepper motor steps from the fully retracted position. When fully inserting and retracting the motor, approximately 25 steps were lost out of approximately 57,000, resulting in a position uncertainty of about $13 \mu \mathrm{m}$. The LVDT used for angular positioning did not show this effect.

At the extreme right of Figure 3.4, a brown box that contains a five sided prism called a pentaprism is shown. It is used to reflect a laser beam through the vacuum chamber window onto the crystal surface, to measure the crystal angle. The measurement of the angle will be discussed in the next section.

The hodoscope made from two scintillators read with photomultiplier tubes (PMTs) is shown in dark blue in Figure 3.4. It is discussed in Section 3.1.5.

\subsubsection{Laser System}

To measure the angular displacement of the crystal with respect to the axis of the beam pipe, a laser is reflected off of a face of the crystal. The deflection of the 
laser measures the crystal angle.

The laser is a $0.5 \mathrm{~mW}$ Helium-Neon (HeNe) laser with a wavelength $632.8 \mathrm{~nm}$. It is placed $4.157 \mathrm{~m}$ from the pentaprism that reflects the laser beam onto the crystal face. The beam is then retro-reflected from the crystal surface, back through the prism to a screen placed just above the laser as shown in Figure 3.1. A CCD camera is used to image the screen. A frame grabber digitizes the image, computes the projection of the laser spot on the horizontal axis, and calculates the horizontal centroid of the laser spot. The centroid position is proportional to the angle of the crystal.

The camera is calibrated by measuring the distance between fiducials placed $1 \mathrm{~cm}$ apart on the screen in terms of the number of CCD pixels. The fiducials are covered after calibration, so that they do not interfere with the centroid measurement.

The crystal angle is given by

$$
\theta_{c}=\frac{x-x_{o f f}}{2 d}
$$

where $d$ is the optical path length from the screen to the crystal face and $x-x_{o f f}$ is the distance of the laser spot from the zero angle position, measured with the camera. The optical path length is defined as the integral of the index of refraction over the path of the laser.

$$
d=\int n(s) \mathrm{d} s \approx 4.6 \mathrm{~m}
$$

where $s$ is the location along the path and $n(s)$ is the local index of refraction. It is a necessary correct for the refractive index of the pentaprism, which has an optical path length of $145.48 \mathrm{~mm}$. The optical path length is also corrected for changes in the crystal transverse position.

Unfortunately, the crystal angle could not be surveyed when the vessel was 
installed in RHIC because of space and time constraints. So the crystal was surveyed in the vessel, outside the tunnel before installation in the ring. The voltage on the LVDT for the angle was recorded during the survey. After installing the crystal vessel, the crystal angle was adjusted so that the LVDT voltage was the same as during the survey. The laser was then aligned. The position of the laser spot on the screen then corresponded to the crystal angle during the survey to within the precision of the LVDT. The crystal angle was varied to calibrate the LVDT, while recording the LVDT voltage and laser centroid. The calibration is shown in Figure 3.6. The LVDT is less sensitive to angular changes than the laser centroid.

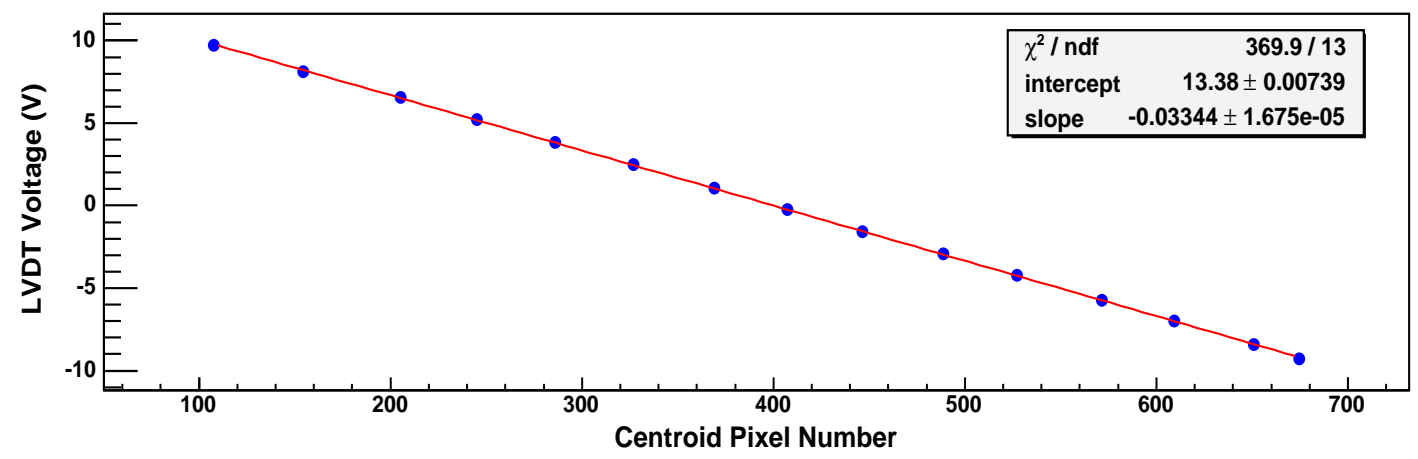

Figure 3.6: Calibration of the LVDT to centroid pixel number. The error bars are smaller than the point size. The line is fit.

Early in commissioning before the FY2001 run it was found that the laser spot changed shape while moving from left to right across the screen as shown in Figure 3.7. In particular, the right side was darkening and disappearing as the spot moved to the right. The left edge, however, seemed to remain unchanged. This skewed the centroid to the left of the spot and affected the reading of the angle. To compensate for this effect, several laser projections and centroids were measured at various locations across the screen. The projection looks like "sloped flat top" and was fit to 


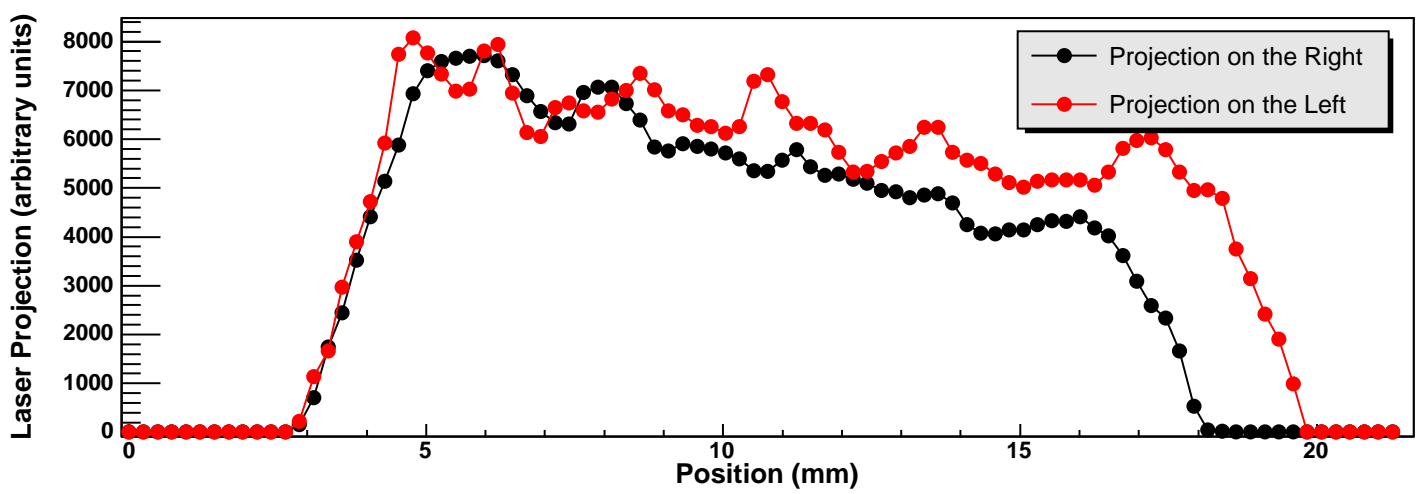

Figure 3.7: Comparison of laser spot projections on the left and right side of the screen.

$$
f(x)=\left\{\begin{aligned}
A \exp \left[-\frac{\left(x-x_{0}+a\right)^{2}}{2 w^{2}}\right]+D & : \quad x<x_{0}-b \\
B\left(x-x_{0}\right)+C+D & : \quad x_{0}-b<x<x_{0}+c \\
F \exp \left[-\frac{\left(x-x_{0}-a\right)^{2}}{2 w^{2}}\right]+D & : \quad x>x_{0}+c
\end{aligned}\right.
$$

where $x_{0}$ is the center position of the projection, $a$ is the distance of the side gaussian from the centroid, $w$ is the width of the gaussian, $b$ and $c$ are the distances from $x_{0}$ to the start of the gaussians, $A$ and $F$ are the height of the gaussians, and $D$ is a constant offset. The parameters $B$ and $C$ are given by

$$
\begin{gathered}
B=\frac{1}{b+c}\left(A \exp \left[-\frac{(b-c)^{2}}{2 w^{2}}\right]-F \exp \left[-\frac{(a-c)^{2}}{2 w^{2}}\right]\right) \\
C=\frac{1}{b+c}\left(c A \exp \left[-\frac{(b-c)^{2}}{2 w^{2}}\right]+b F \exp \left[-\frac{(a-c)^{2}}{2 w^{2}}\right]\right)
\end{gathered}
$$

to assure continuity of the function. A typical projection with a fit is shown in Figure 3.8.

The left edge is chosen to be

$$
x_{l e}=x_{0}-a-w .
$$




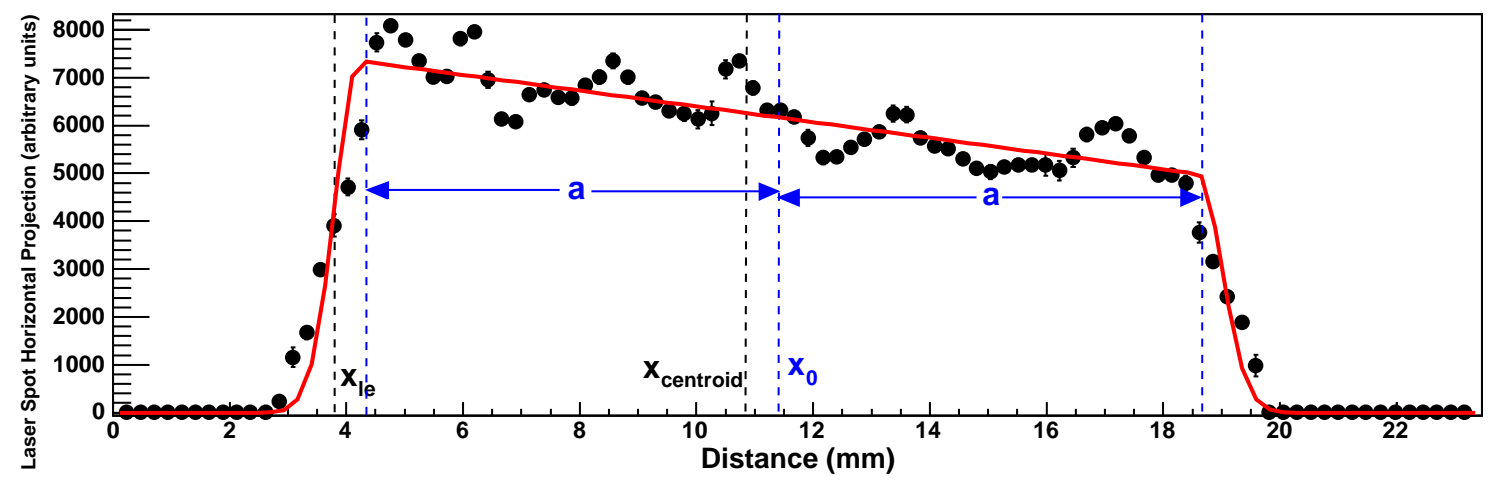

Figure 3.8: Typical laser projection, showing relevant parameters.

This was plotted as a function of centroid and fit to a line, as shown in Figure 3.9. This is used to compute left edge from the centroid. The position of the left edge is then used to monitor the laser deflection and thus the crystal angle.

To determine the absolute uncertainly in the crystal angle, the crystal angle was adjusted to match the LVDT voltage found during the survey, and the laser position recorded. The crystal angle was varied slightly, keeping the LVDT voltage constant, and the laser position recorded. This was repeated many times. These measurements show that the uncertainty in the knowledge of the absolute crystal angle from the LVDT voltage is $80 \mu \mathrm{rad}$.

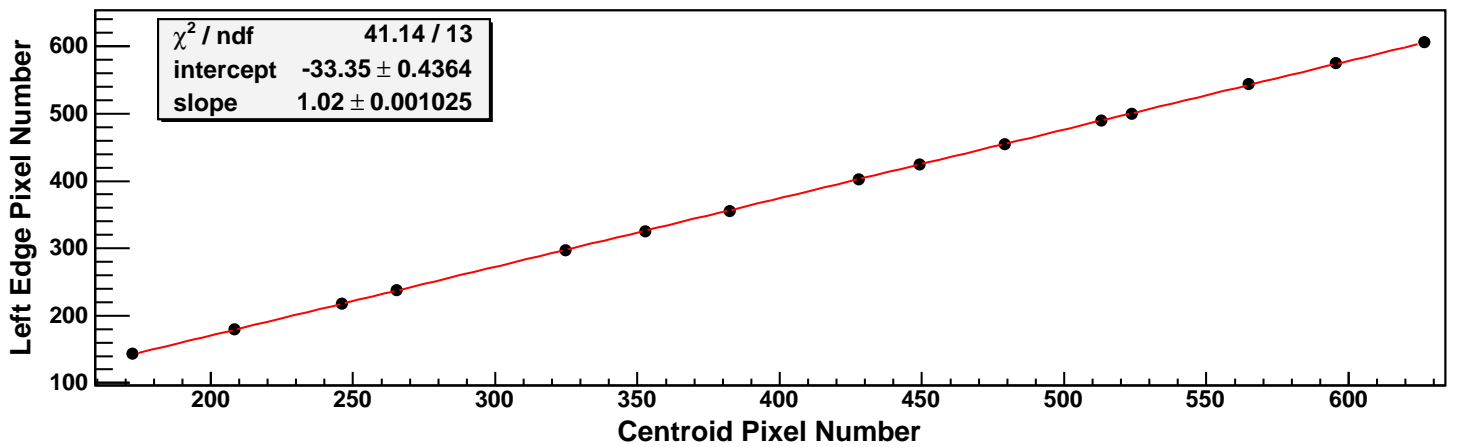

Figure 3.9: Calibration of laser left edge to centroid pixel number. The error bars are smaller than the point size. The line is a fit. 
However, relative changes can be measured with greater precision. By repeatedly measuring the laser centroid at one angle and repeating this for different angles, we determined relative changes in the crystal angle to have an error of approximately $25 \mu \mathrm{rad}$. The precision is limited by the camera resolution and the variations of the laser spot. However, the inchworm is able to move the crystal by as little as $\approx 30 \mathrm{nrad}$.

\subsubsection{Copper Scraper}

The copper scrapers are $450 \mathrm{~mm}$ long inverted L shaped copper blocks. The vessel is shown in Figure 3.10. Each block is fixed inside of the vessel. The vessel has the ability to move horizontally and vertically and to rotate about the vertical axis. Each degree of freedom is controlled by a stepper motor. The position is monitored with an LVDT. The yellow scraper has a phosphor flag placed on the vertical portion of the copper to image beam striking the scraper.

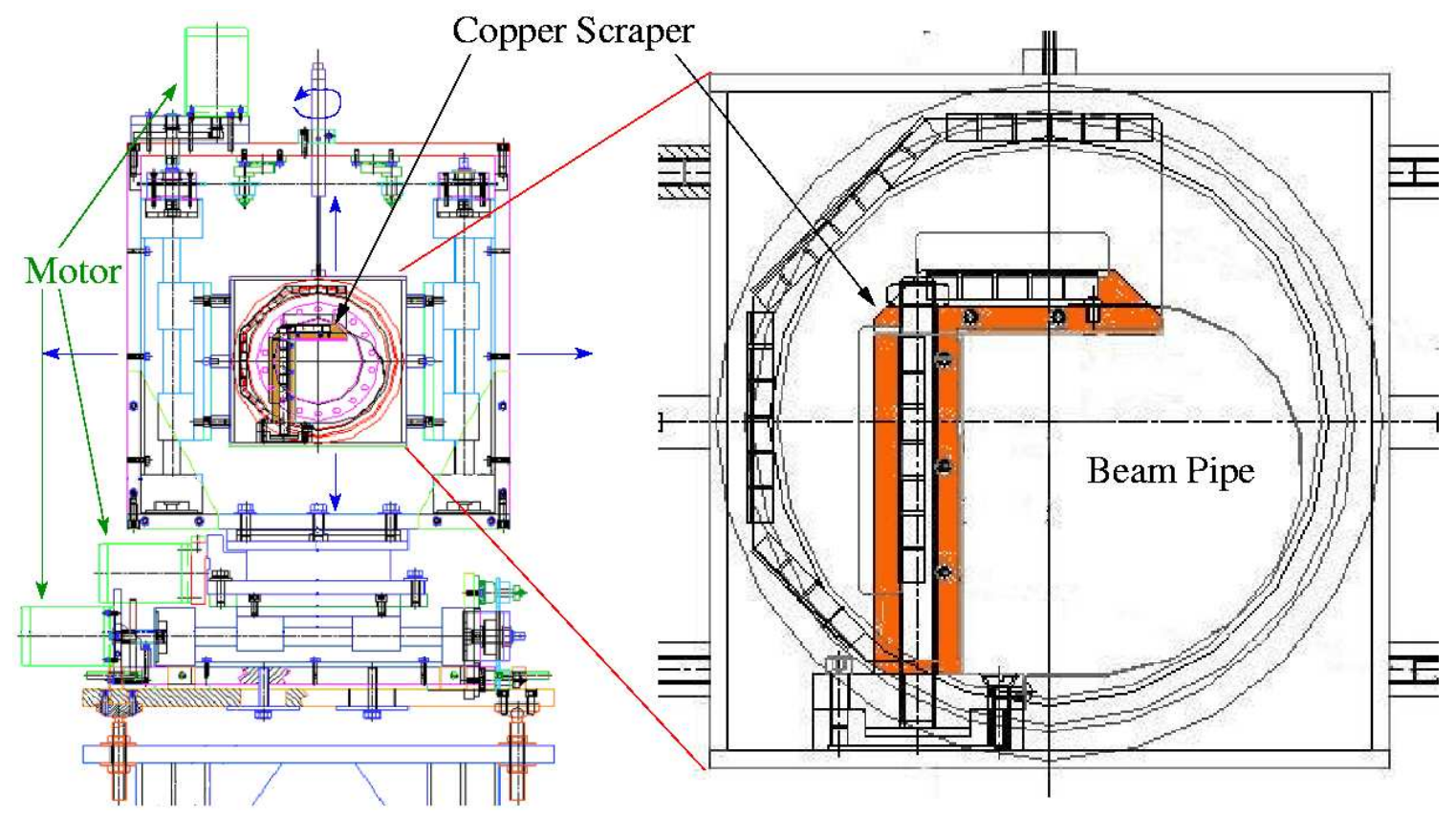

Figure 3.10: Copper Scraper vessel. The scraper is highlighted. 
We decided to count stepper motor steps to determine the scraper position and to use the LVDTs as a redundant measure, because of the thermal drifts of the LVDTs that were seen with the crystal vessel,

\subsubsection{Detectors}

The RHIC collimation system has three detection systems to measure local beam losses, the PIN diodes, the crystal hodoscope and the dedicated BLMs.

\section{PIN Diodes}

The RHIC collimation system includes a total of 16 PIN diode detectors, as shown in Figure 3.1. They are used to measure beam losses due to the crystal collimator and scrapers. The PIN diodes are more sensitive to beam loss than the BLMs. This makes them more useful when the scrapers and crystal are in the beam halo.

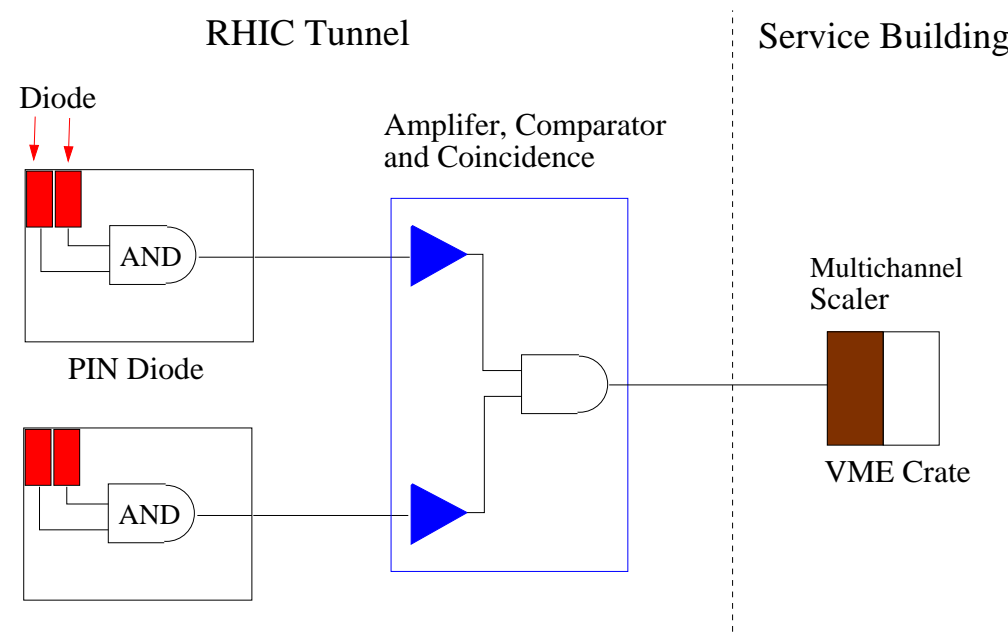

Figure 3.11: PIN Diode Block Diagram

Each set of PIN diodes is mounted in a ring with a central diameter of $281 \mathrm{~mm}$. The downstream PIN diodes are $2.42 \mathrm{~m}$ downstream of the end of each scraper in 
each ring and the upstream PIN diodes are $2.73 \mathrm{~m}$ upstream of the yellow scraper. Each PIN diode loss monitor consists of two diodes placed back to back. The diodes are Hamamatsu S3590-03 diodes used at CERN and DESY [52]. When a minimum ionizing particle (MIP) passes through each diode, it loses energy via ionization generating a current pulse in the diodes. Each pulse is amplified and applied to a comparator. The coincidence of these comparator pulses is one count of the PIN diode and is free from the individual diode dark currents [53]. A scaler is used to count the number of pulses. The maximum counting rate of the PIN diodes is $1 \mathrm{MHz}$. Figure 3.11 shows a block diagram of the PIN diode electronics. A system of two diodes in coincidence mode is referred to as "one" PIN diode.

\section{Hodoscope}

The hodoscope, shown in Figure 3.4, is used to measure the large angle scattering of particles from the crystal collimator. The geometry of the hodoscope is shown in Figure 3.12. It sits at an angle of $40^{\circ}$ to the beam axis with the center of its

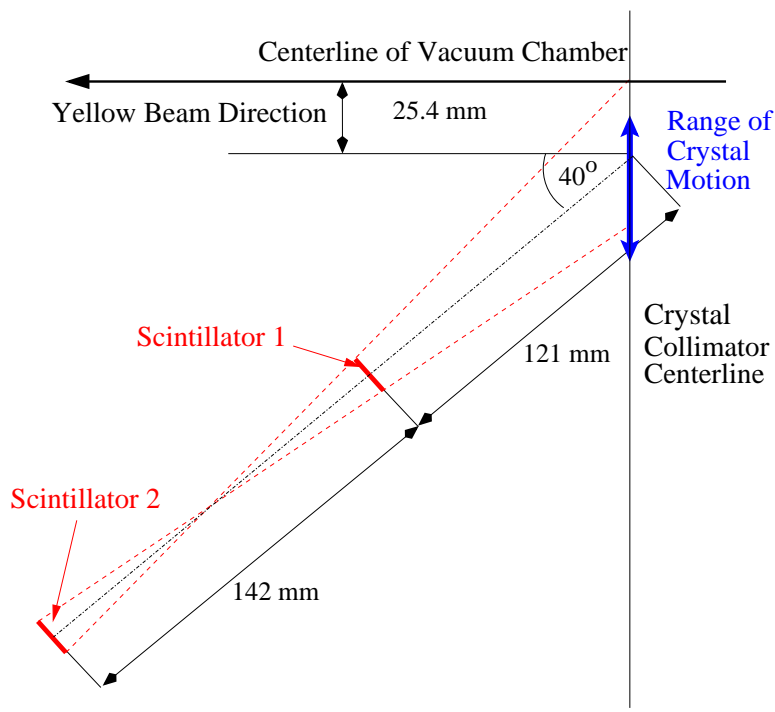

Figure 3.12: Hodoscope geometry. Figure is drawn to scale. 
acceptance $25.4 \mathrm{~mm}$ to the inside of the vacuum chamber centerline on the crystal collimator axis of motion. This ensures that the hodoscope can detect particles from the crystal collimator throughout its operating range. The motion of the crystal collimator is shown in blue in Figure 3.12. Dotted red lines denote the acceptance of the hodoscope.

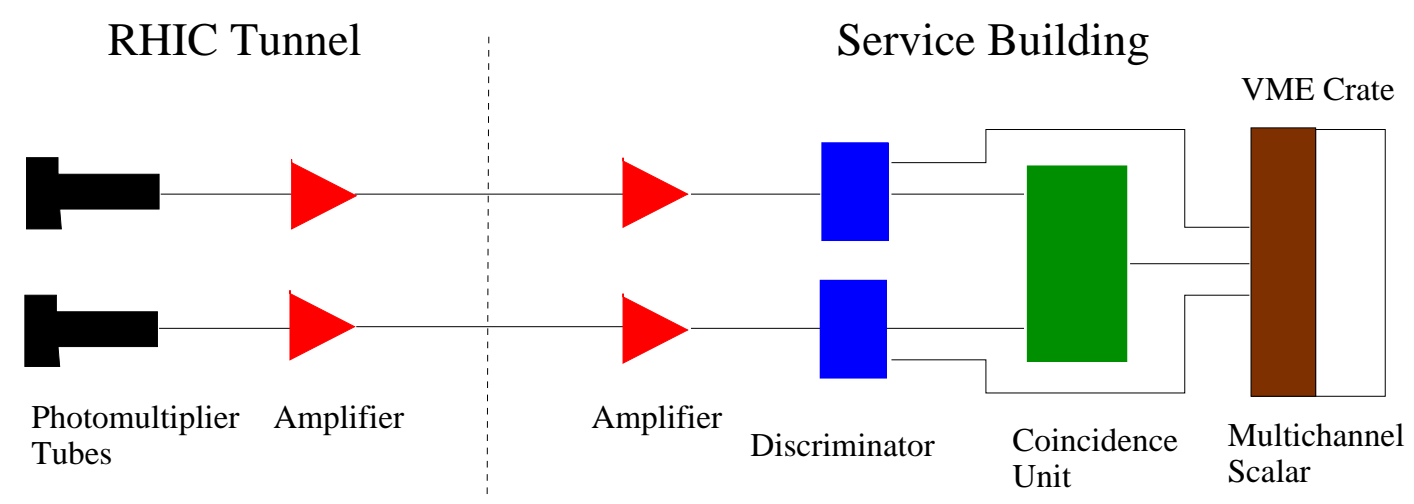

Figure 3.13: Circuit for Hodoscope

Each scintillator is read with a PMT. The signal from each PMT is amplified in the tunnel and transmitted to the service building. It is necessary to amplify the signals again in the service building because of the long length of cable needed. The amplified signals are fed to discriminators and then to a coincidence unit. The discriminator and coincidence signals are read by a VME based scaler. The circuit diagram is shown in Figure 3.13.

\section{Beam Loss Monitors}

The eight argon ionization BLMs dedicated to the collimation system are placed between the scrapers and the spin rotator magnets, as shown in Figure 3.1. They are used to measure large losses from the collimators, and are useful in comparing losses at the collimators with losses at other areas of the ring. The BLMs for the collimation system are not connected to the beam permit system because beam losses 
at the collimators are not a safety hazard for RHIC components. The environmental risk from residual radioactive nuclei is minimized by a waterproof liner around the collimation section of the tunnel.

\section{Cameras}

The collimation system has two cameras. The first is a CCD camera that monitors the laser spot used to measure the crystal collimator angle. The second camera is used to image the phosphor flag on the yellow scraper to monitor the beam impacting the scraper. Each camera is fed into an RF multiplexer. The multiplexer then communicates to an RF switch which is controlled via PC. This switch diverts the video signal to a selected television monitor in the control room or VME based frame grabber. The frame grabber digitizes the signal and allows the image to be processed by the front end computer and recorded.

\subsection{Beam Parameters}

It is useful to discuss the Twiss parameters in the region of the collimators. Table 3.1 lists the design Twiss parameters at the collimators for each $\beta^{*}$ used at

Table 3.1: Design Twiss Parameters at Collimators for various $\beta_{P H E N I X}^{*}$

\begin{tabular}{|c|c||c|c|c|c||c|c|c|c|}
\hline$\beta^{*}(\mathrm{~m})$ & Plane & $\beta_{c}(\mathrm{~m})$ & $\alpha_{c}$ & $D_{c}(\mathrm{~m})$ & $D_{c}^{\prime}$ & $\beta_{s}(\mathrm{~m})$ & $\alpha_{s}$ & $D_{s}(\mathrm{~m})$ & $D_{s}^{\prime}$ \\
\hline \multirow{2}{*}{5} & $\mathrm{H}$ & 242 & -4.9 & 0.103 & 0.0055 & 171 & -4.13 & 0.060 & 0.0055 \\
& $\mathrm{~V}$ & 79 & -3.0 & 0 & 0 & 40 & -2.03 & 0 & 0 \\
\hline \multirow{2}{*}{3} & $\mathrm{H}$ & 391 & -8.3 & -0.012 & 0.0032 & 272 & -6.9 & -0.037 & 0.0032 \\
& $\mathrm{~V}$ & 132 & -4.9 & 0 & 0 & 67 & -3.4 & 0 & 0 \\
\hline \multirow{2}{*}{2} & $\mathrm{H}$ & 581 & -12.7 & -0.152 & 0.0002 & 399 & -10.6 & -0.153 & 0.0002 \\
& $\mathrm{~V}$ & 200 & -7.2 & 0 & 0 & 103 & -5.2 & 0 & 0 \\
\hline \multirow{2}{*}{1} & $\mathrm{H}$ & 1155 & -26.5 & -0.864 & -0.0162 & 778 & -21.7 & -0.737 & -0.0162 \\
& $\mathrm{~V}$ & 402 & -14.2 & 0 & 0 & 211 & -10.2 & 0 & 0 \\
\hline
\end{tabular}

The values listed are for the yellow ring. The corresponding values for the blue ring are similar. The $c$ subscript denotes the values at the crystal location, the $s$ subscript denotes the values at the scraper. 

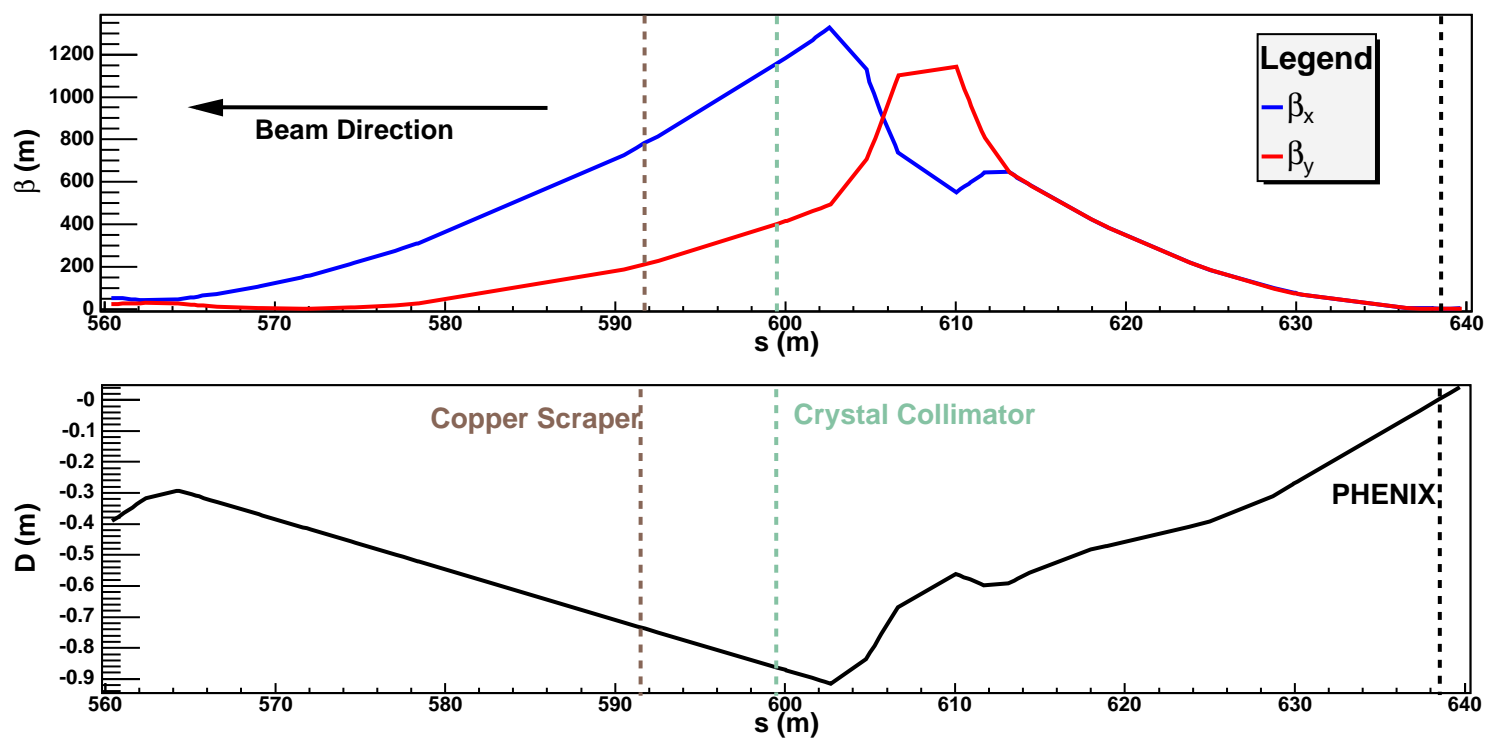

Figure 3.14: Design Horizontal $\beta$ functions and dispersion function near the Crystal Collimator for $\beta^{*}=1 \mathrm{~m}$. The $\beta$ and dispersion functions for the blue ring are similar.

PHENIX. The drift space that the collimators occupy is immediately downstream of the outgoing triplet for the PHENIX detector in each ring. Because of this, any change in the $\beta^{*}$ at PHENIX greatly changes the Twiss parameters at the collimators. In addition, because of the placement of the dipole correctors, any steering done in PHENIX has an effect on the beam location at the collimators.

Figure 3.14 shows the design $\beta$ functions and dispersion through the collimation section in the yellow ring for $\beta^{*}=1 \mathrm{~m}$. The large $\beta$ functions and small dispersion function indicate that betatron collimation will be effective, and momentum collimation will not work well.

\subsection{Controls Software}

The software to control the collimators centers around two main programs. The first, "CrystalCollimator", is used to control the crystal collimator. The second, "Collimator", controls the scrapers in each ring. In addition, a general purpose 
program called "PET" can be used to control the collimation system.

\subsubsection{PET Page}

The Parameter Editing Tool (PET) program allows the user to edit individual parameters for a particular system [54]. PET allows access to low level parameters of each RHIC system. It is most useful for experts debugging a system or controls software. It is not very useful for operations because it requires expert knowledge in each system to use it. PET was used to control both the crystal collimator and the scrapers until the control programs were written. Figure 3.15 shows the PET page for the blue scraper.

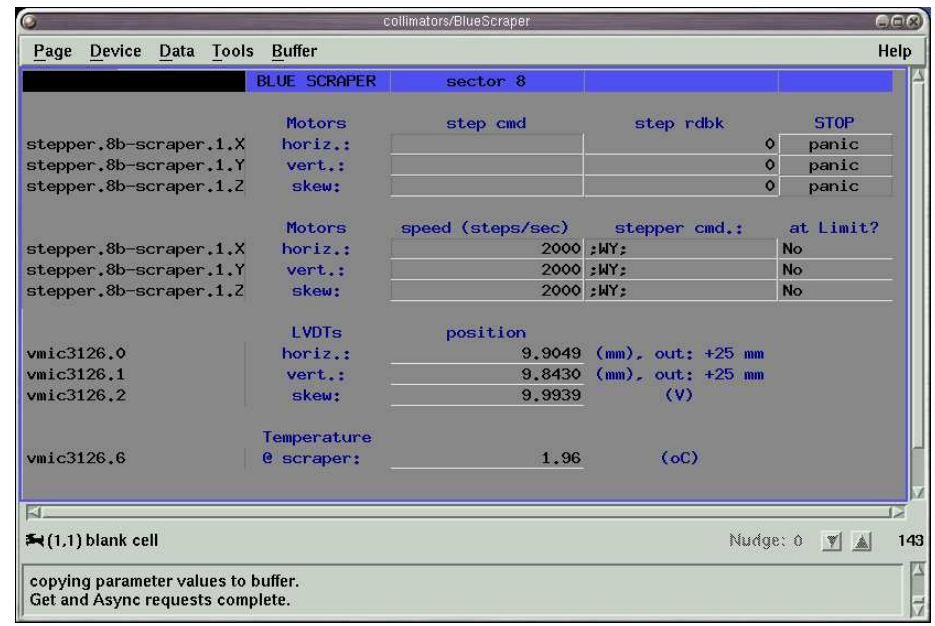

Figure 3.15: Blue Scraper PET Page

\subsubsection{Crystal Control Program}

The software to control the crystal was written by Lee Hammons [55]. A screen shot is shown in Figure 3.16. The left side of the GUI contains the controls for the crystal horizontal position. The user has the ability to enter a desired location, or to move the crystal collimator by a set number of millimeters. A button is provided for 


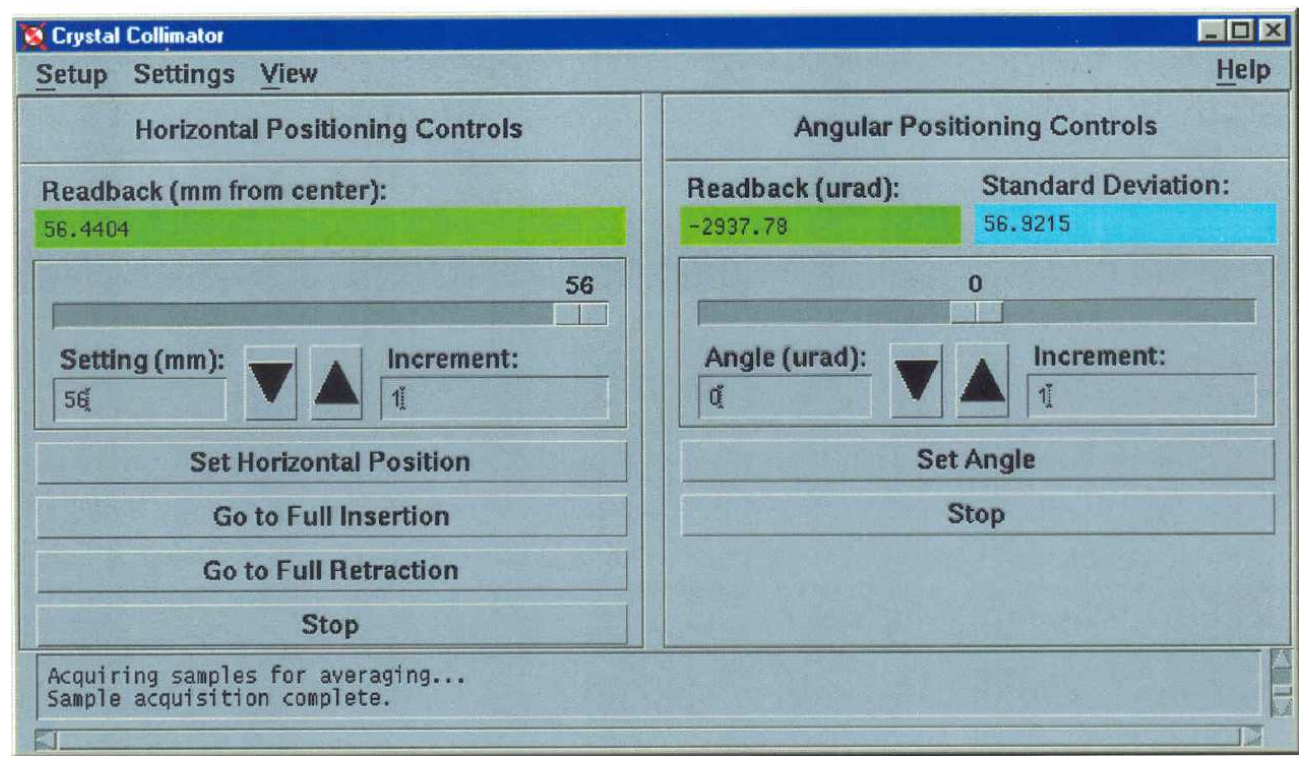

Figure 3.16: Crystal Collimator Control Program

crystal retraction. A "panic" button is available for emergency stops of the crystal motion.

The right side of the the window provides the interface for the crystal angle control with a similar interface. A continuous readback of the angular position with an estimated error is provided. To measure the crystal angle, the program takes an average over 10 laser centroid positions from the frame grabber and converts this into an angle as described in Section 3.1.3. The error is calculated from the standard deviation of the centroids. Because reading the laser centroid is slow, the LVDT is used for monitoring large movements of the angle. The laser is used when the LVDT indicates that the angle is close to the desired angle.

Another feature, accessible from a menu, provides the ability to scan the crystal angle across a programmable range. The user can specify the range of angles, the approximate step size, and the dwell time per step. This feature was used during the crystal scans discussed in Chapter 5. 


\subsubsection{Scraper Control Program}

The scraper control program was written by Wenge $\mathrm{Fu}[56]$. A screen shot of the program is shown in Figure 3.17. The program controls both scrapers, but only allows the control of one scraper at a time. "Panic" buttons immediately stop the scraper movement for any particular motor. Home buttons return each motor to the fully retracted position. A convenience display in the upper right corner shows the collimator position relative to the beam. The beam spot, located near the center of the picture, is scaled to the beam size measured by the ionization profile monitors (IPMs). Its position is determined from the BPMs. The graph at the bottom left of the window plots the beam and scraper positions as a function of time. The lower right graph plots the distance between the scraper and the beam in units of the rms beam size.

A menu option opens a series of windows for the user that display the PIN diode

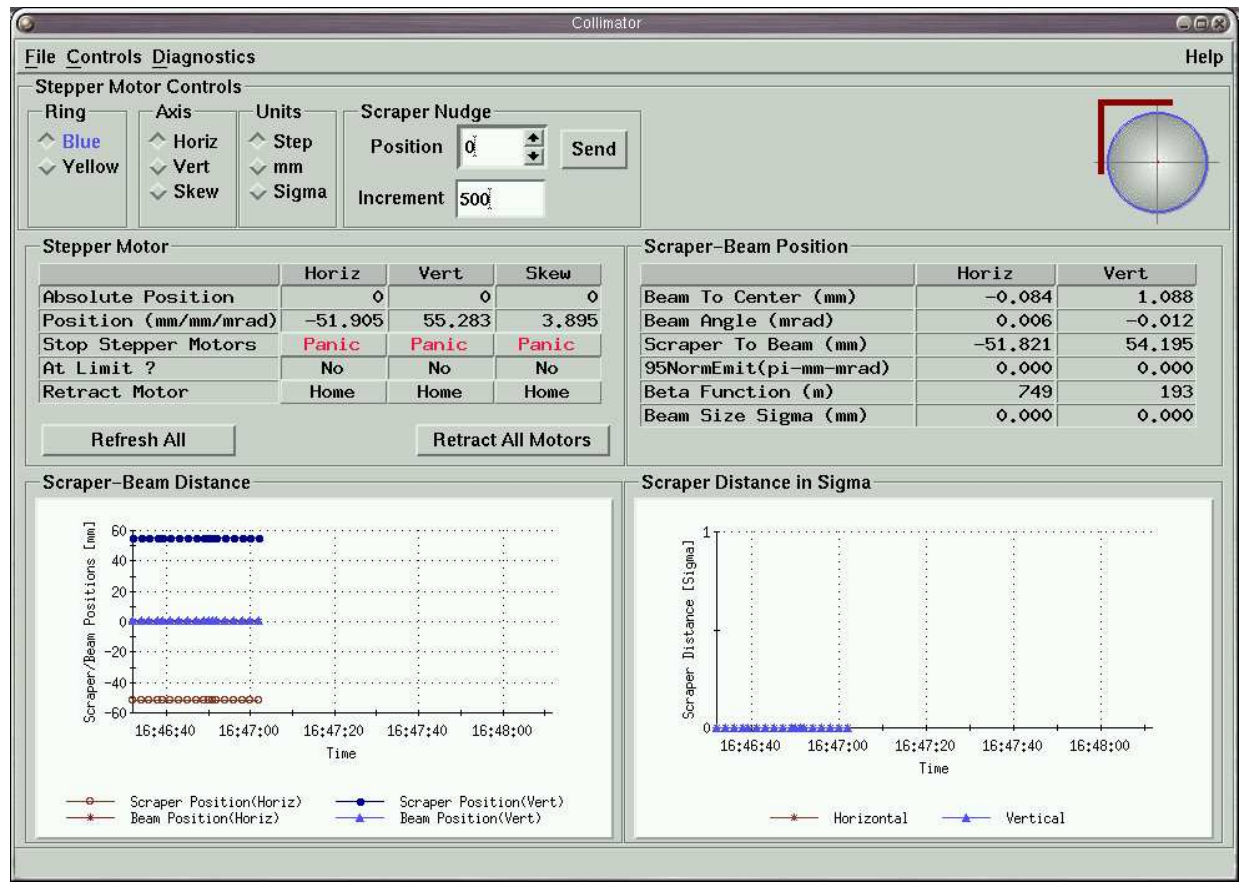

Figure 3.17: Scraper Control Program 
count rates, beam currents, and selected backgrounds for all experiments. Thus the user can adjust the scraper positions and angles to minimize the backgrounds at all experiments, while assuring good beam lifetime. Other menu options open PET pages and other monitors.

\subsection{Data Logging}

Two programs are used the record the data from the collimation system. The first is the "Logger" [57], a utility program used at RHIC to record specified data during RHIC operation. The data can then be time correlated for analysis. The PIN diode rates, hodoscope rates, BLMs, all stepper motor positions, LVDTs, and selected Beam Position Monitors are all routinely logged for the collimation system.

A program called General Purpose Monitor (GPM) is also used to record data [58]. This program, like Logger, records a time series of data for analysis. GPM records all of the collimator instrumentation discussed in this chapter. The data is saved in the Self Describing Data Sets (SDDS) file format [59]. GPM is used to record data during crystal commissioning and beam studies since the data requires less processing before analysis.

\subsection{Data Analysis Program}

The ROOT Data Analysis Framework is used for data analysis [60]. ROOT is a set of $\mathrm{C}++$ class libraries surrounding a $\mathrm{C}++$ command interpreter CINT, which is used as the command interface to the ROOT system. All ROOT commands are $\mathrm{C}++$ program statements. ROOT libraries include classes for graphics, data analysis and visualization, linear algebra, networking, and various other functions. It is possible 
to write additional libraries that can be incorporated to the local environment. The flexibility of $\mathrm{C}++$ allows ROOT to handle large data sets efficiently.

\subsection{Commissioning}

For the first RHIC physics run in 2000, only the copper scrapers, PIN diodes and BLMs were installed in both rings. The focus for the collimation system was commissioning, and gaining experience with the system. Because of the lower beam intensities and energy during this run, the experimental backgrounds were small. This made it possible to commission the collimation system in parallel with a store without affecting the experiments.
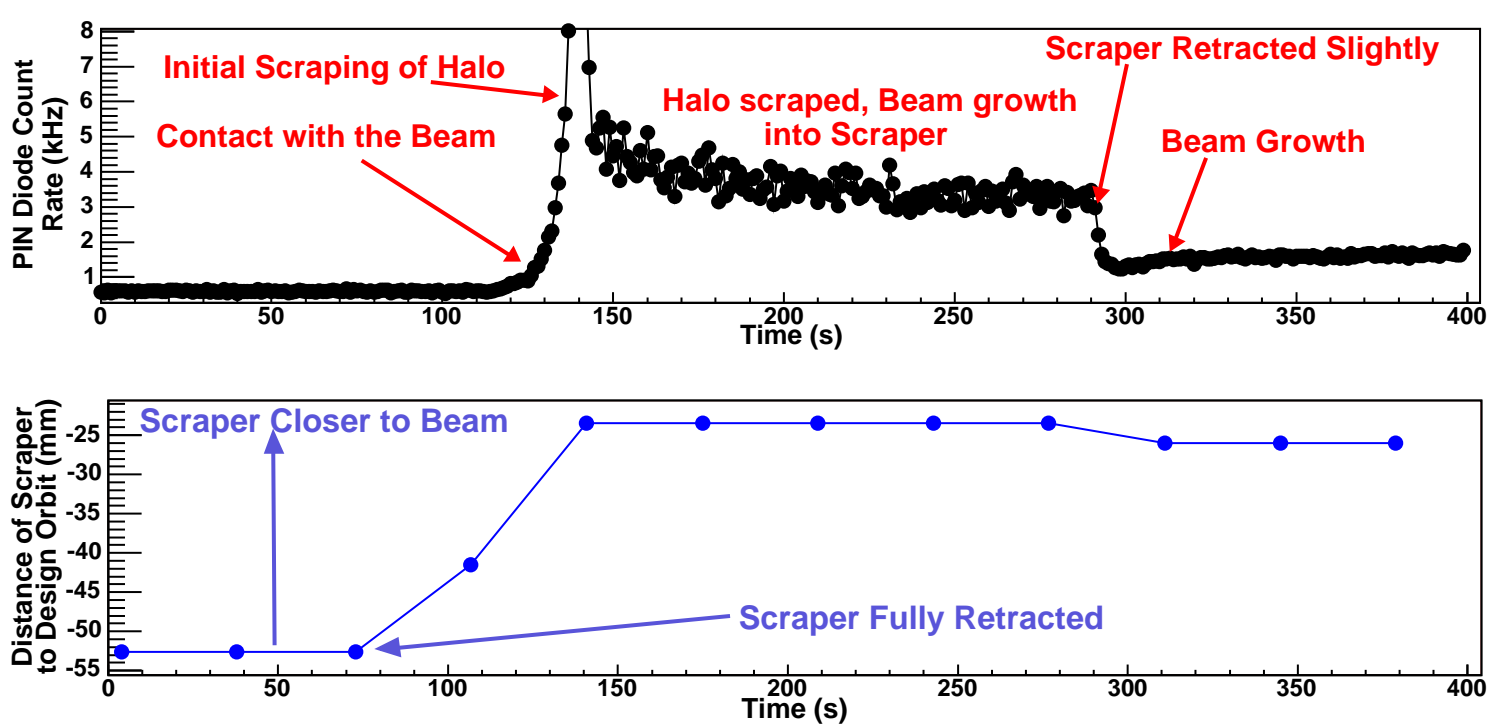

Figure 3.18: Effect of the copper scraper on the RHIC gold beam in the yellow ring.

Figure 3.18 shows the effect of the yellow copper scraper on the RHIC gold beam as seen from a PIN diode downstream of the scraper. The scraper encounters halo when moved into the beam which is promptly scraped away. This is the large spike at $140 \mathrm{~s}$. With the scraper stationary, the beam just touches the edge of the 
scraper. Particles near the beam edge diffuse into the scraper by the mechanisms described in Section 1.3. When the scraper is slightly retracted the PIN diode loss rate decreases, then grows slightly. This growth occurs after the beam halo diffuses out to touch the scraper again.

Only a very small fraction of the beam is removed by the scraper, so the lifetime is unaffected by the action of the scraper. This is to be expected when scraping the beam halo. However, it is possible to scrape away the entire beam. This was done with one bunch at injection to correlate the PIN diode rates with a beam loss rate. A byproduct of this is a rough measurement of the transverse beam profile. These are detailed in Chapter 6.

The crystal collimator was installed, during the winter shutdown of 2000. The crystal collimator was commissioned and studied during the 2001 run. The results of these measurements are detailed in Chapter 5. The scrapers were used operationally for the first time for collimating on the ramp and abort gap cleaning during the same run. During the 2003 run the scrapers were used during stores to significantly reduce experimental backgrounds. This is discussed in Chapter 6 . 


\section{Chapter 4}

\section{Simulations}

Simulation is important when estimating various aspects of accelerator performance. A variety of problems do not lend themselves to simple theoretical models because they contain statistical, chaotic, or nonlinear behavior. With tracking simulations it is possible to include an arbitrary number of effects, limited only by computation time.

Estimating the efficiency of a collimation system is one such case. The results presented in Chapter 2 contain only enough detail to estimate performance. Effects such as particle scattering in a bent crystal, nonlinearities of the accelerator, and particle diffusion are not included in the theoretical model because of their complexity.

This chapter outlines all of the various programs used to simulate the RHIC collimation system and presents results of those simulations. Results of simulations of the collimation system upgrade are presented in Chapter 7 . 


\subsection{Software}

In order to simulate the effect of a bent crystal on the beam halo, it is necessary to use many different programs:

- CATCH [61] to simulate the bent crystal.

- K2 [62] and ACCSIM [63] to simulate proton scattering in the copper scraper.

- UAL [64] for simulation of distributed losses in RHIC.

- scan for channeling efficiency simulations.

Each of these codes and their role in simulations of the RHIC collimation system are described below.

\subsubsection{CATCH}

Section 2.1 outlines the theory of bent crystal channeling. In order to simulate crystal channeling, a Monte Carlo simulation named Capture And Transport of CHarged particles (CATCH) was written by Valery Biryukov. Given an initial particle distribution, CATCH transports particles through a crystal lattice. CATCH uses the verlet algorithm [65] to track particles through the interplanar potential. Nuclear interactions, electron scattering, and energy loss are included. CATCH is able to simulate all particle species. Crystal lattices are limited to the (110) and (111) orientations of diamond lattices.

Figure 4.1 shows a typical simulation using CATCH. The left figure shows the horizontal particle phase space at the entrance of the crystal. The edges of the crystal and the range of angles that are within the acceptance of the crystal are shown. The right figure shows the same phase space at the exit of the crystal. The channeled 

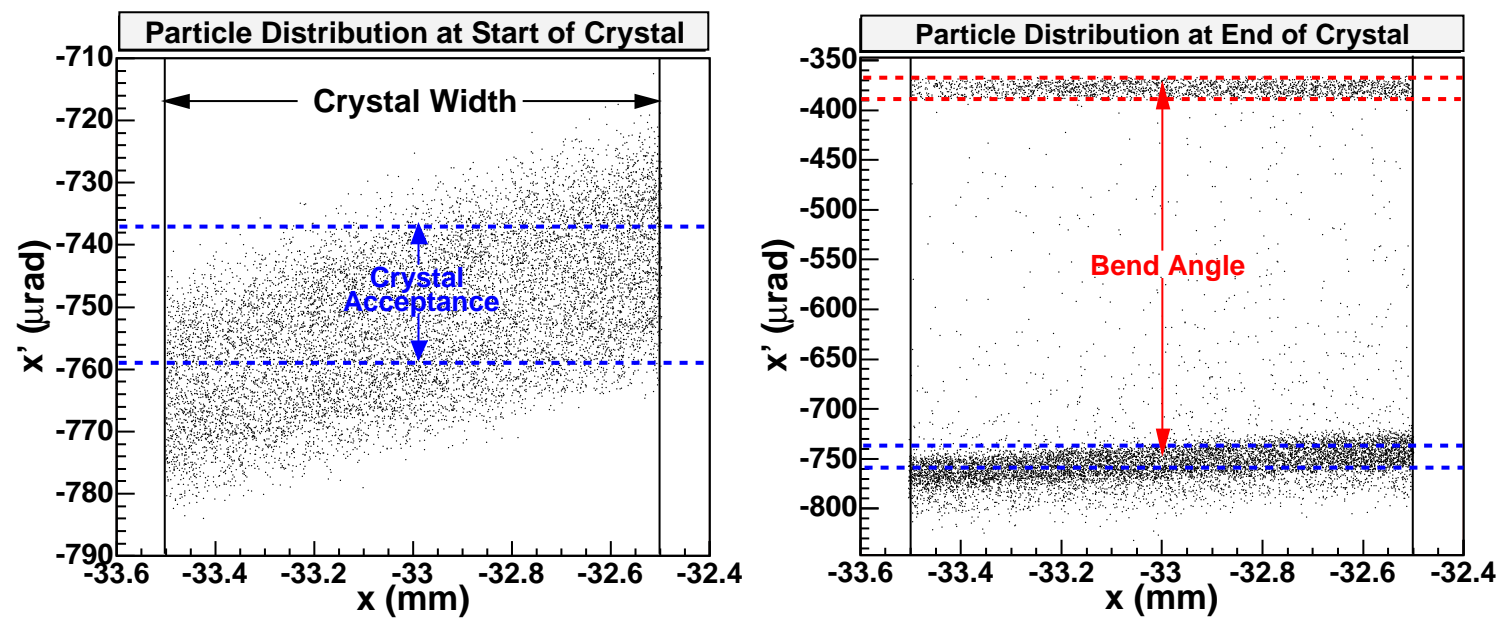

Figure 4.1: Simulation of crystal channeling of particles hitting a silicon crystal. The left figure shows the horizontal phase space at the entrance to the crystal, indicating the crystal angular acceptance. The right figure shows the same phase space at the end of the crystal. The blue and red dashed lines indicate the angular acceptance and divergence of the crystal.

particles appear between the red lines. They receive an angular kick approximately equal to the bend angle of $370 \mu \mathrm{rad}$. Particles that are not channeled, but scatter through the crystal appear at the bottom of the plot. Particles that scatter out of the channel and do not get the full angular kick of the crystal appear at intermediate angles. In the vertical phase space, particles are tracked as in a drift with additional scattering.

\subsubsection{K2}

It is necessary to include the effects of the proton scattering inside of the copper scraper when simulating the efficiency of the collimation system as a whole. Scattering in the scraper changes the angle and energy of the particles as outlined in Section 2.2. A Monte Carlo simulation named K2 was written by Jean-Bernard Jeanerette to simulate scattering inside of the copper scrapers. K2 includes multiple Coulomb scattering, diffractive scattering, inelastic nuclear scattering and energy loss in amor- 
phous material. K2 is tailored to simulate protons only because of the complexity of nuclear interactions.

\subsubsection{ACCSIM}

ACCSIM was originally written by Fred Jones to simulate intense proton beams. Portions of the ACCSIM code were ported to $\mathrm{C}++$ by Nikolay Malitsky for use in UAL. Among the portions of ACCSIM in UAL is the ability to track particles though a collimator. With this addition, it is possible to write a self contained UAL simulation of the scrapers without the extra modules to send information to, and to receive information from, K2.

ACCSIM contains the same physics as K2 with the exception of diffractive scattering and is tailored for simulating protons as well. ACCSIM has a variety of apertures to choose from to simulate scattering from the edge of the scraper. Different aperture shapes needed to be added to the local version of K2. ACCSIM and K2 are compared in Section 4.4 .

\subsubsection{UAL}

The Unified Accelerator Libraries (UAL) package is used to track particles around the RHIC rings. UAL is based on the Thin Element Accelerator Program for Optics and Tracking (TEAPOT) code [66]. UAL is structured so that other tracking codes, such as ACCSIM, can be included into the accelerator simulation. TEAPOT can be used to track through magnets and TIBETAN [67] can be used for tracking through RF cavities if the user desires. The library source code is written in $\mathrm{C}++$. A PERL interface to the libraries is provided to take advantage of some advantages of PERL, such as the lack of compilation. Figure 4.2 shows the UAL architecture. 


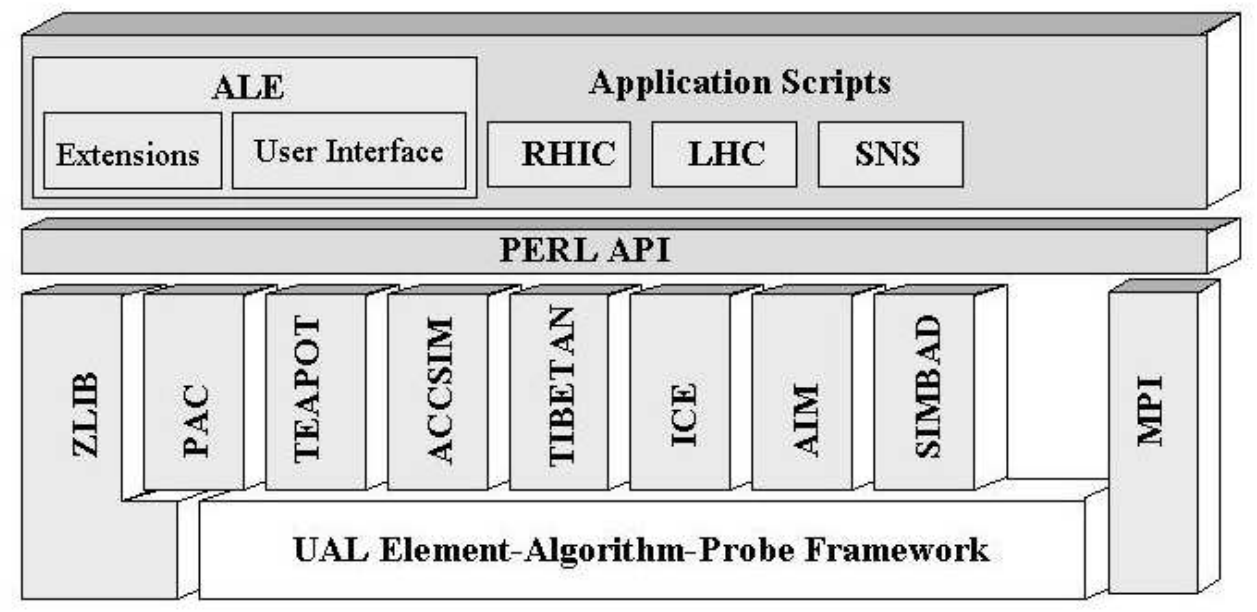

Figure 4.2: UAL Architecture.

Simulation codes outside UAL that are stand alone executables are accessed from the UAL via PERL modules. This is done in the case of CATCH and K2. PERL modules send particles to the appropriate program when the particles hit the crystal collimator or the copper scraper.

It is desirable to integrate simulation and analysis into the same program since most simulation results are written to ASCII file, only to be read by another program for analysis. An interface between ROOT and the UAL achieves this goal. The advantages of not having to compile programs is kept because ROOT interprets $\mathrm{C}++$ code. These $\mathrm{C}++$ scripts can be compiled if speed is an issue.

UAL is integrated into ROOT using the ROOT dictionary generator, rootcint. The ROOT dictionary for each UAL library is linked to the shared object file for the library. This assures that errors are not introduced into UAL while allowing it to be used directly in ROOT scripts. This has been done successfully for a number of the UAL libraries.

A shell has been written named RootShell, modeled after the ALE::UI::Shell written for the PERL interface in order to facilitate the ease of use of the UAL inside 


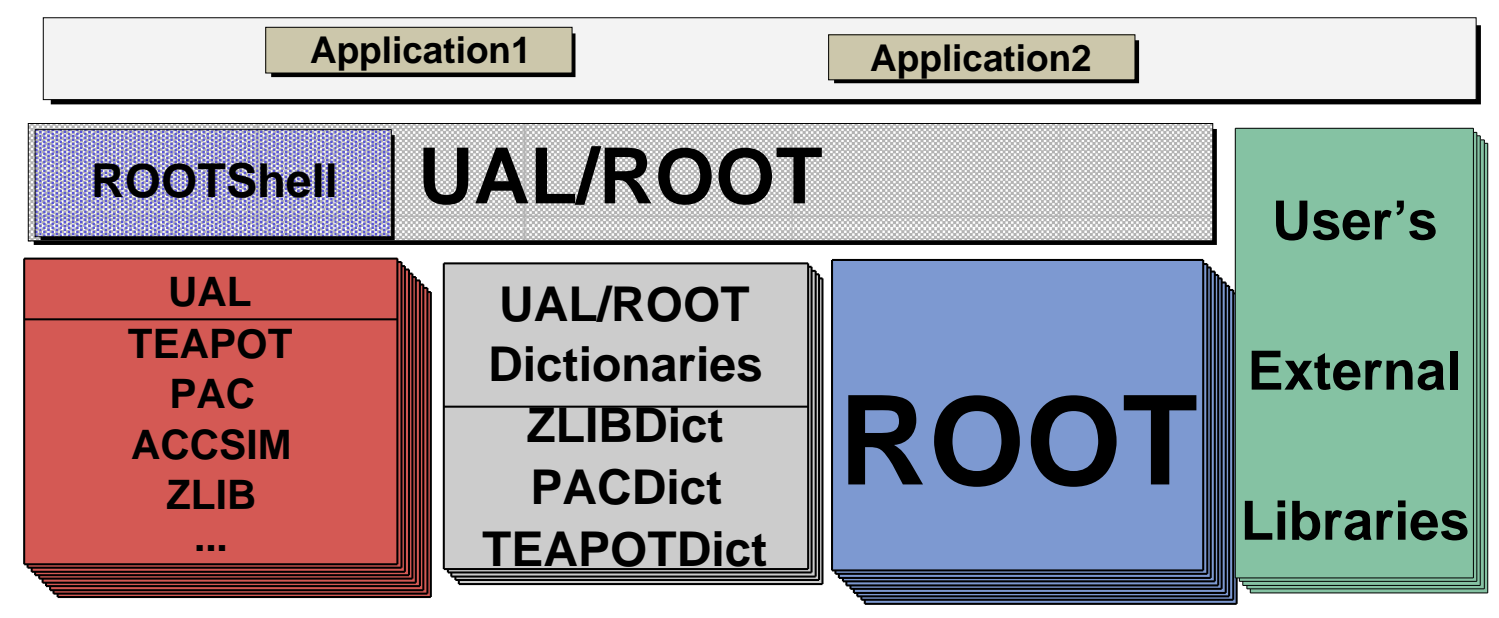

Figure 4.3: UAL/ROOT Architecture.

of ROOT. This shell provides a number of generally used functions and hooks to libraries that have not had a dictionary generated. Some of the functions provided in RootShell are:

1. readSXF - reads an accelerator lattice.

2. setBeamAttributes - defines beam attributes.

3. generateBunch - generates a particle bunch matching Twiss parameters at a particular element.

4. track - tracks particles through a portion of the lattice.

5. multitrack - tracks particles through a lattice for multiple turns.

6. hsteer, vsteer - flattens central orbit.

7. tunethin - adjusts horizontal and vertical tunes.

8. chromfit - adjusts horizontal and vertical chromaticities.

9. decouple - decouples the lattice. 
10. analysis - finds closed orbit and does Twiss analysis.

Other conversion functions facilitate the transfer of UAL classes to ROOT classes for analysis. One example is the bunch2Ntuple function which copies data from a UAL bunch to a ROOT TNtuple for analysis. Examples of how to use these new features are included in the UAL distribution [68]. Figure 4.3 shows the structure of the new UAL/ROOT interface.

\subsection{5 scan}

To simulate what would be seen when the crystal angle is rotated, a $\mathrm{C}++$ program named scan that tracks particles around RHIC for a set number of turns and a range of crystal angles was written. The simulation starts at the crystal after the initial distribution is chosen. Particles that strike the crystal are sent to the CATCH code. The others are tracked past the crystal. Particles are then tracked through a drift to the scraper jaw. Particles that strike the scraper are sent to K2 if they are protons, or are assumed to interact inelastically if they are other ions. Particles not hitting the scraper are tracked to the end of it. A $6 \times 6$ simplectic matrix with no coupling or RF brings all of the remaining particles around to the

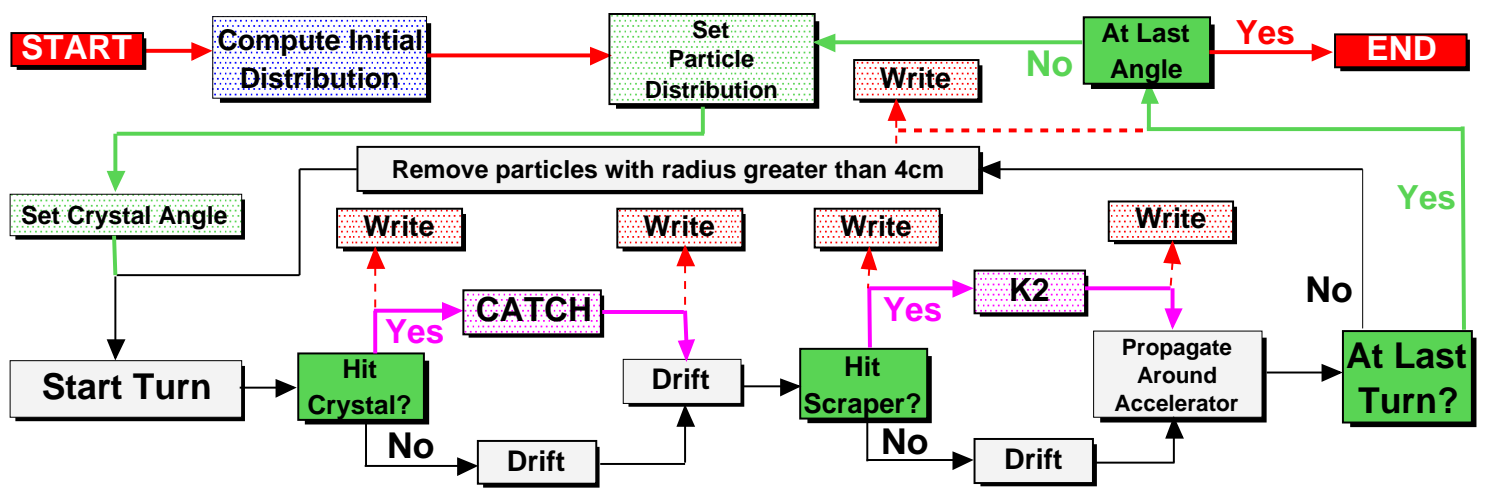

Figure 4.4: Flow chart of scan simulation illustrating the algorithm used to simulate crystal scans. 
crystal again. Particles that have a radius of greater than $4 \mathrm{~cm}$, or inelastically scatter in the crystal or scraper, are no longer tracked. This process is repeated for a set number of turns and crystal angles. A flow chart of the simulation appears in Figure 4.4.

The particle distribution is an exponential distribution in the horizontal action, uniform in the vertical action, and Gaussian in the momentum offset. This reflects the distribution in Equations 2.66 and 2.67. The time difference between particles is chosen to be zero. The distributions are chosen with typical RHIC beam emittances of $15 \pi \mathrm{mm}-\mathrm{mrad}$, and are matched to the transverse phase spaces. The fractional rms momentum spread is $0.13 \%$. In the horizontal plane, only particles that lay on an ellipse that would intercept the crystal are chosen. In this way, particles in the center of the bunch are ignored. No such cut is placed in the vertical plane. At the beginning of the program, the initial distribution is calculated and is used every time the crystal angle changes. A typical distribution is shown in Figure 4.5.

The phase space locations of all of the particles are recorded in a file for the initial distribution, after every turn, and for all particles that enter and leave the crystal or scraper. In addition, if a particle is lost it is recorded to a file. These files
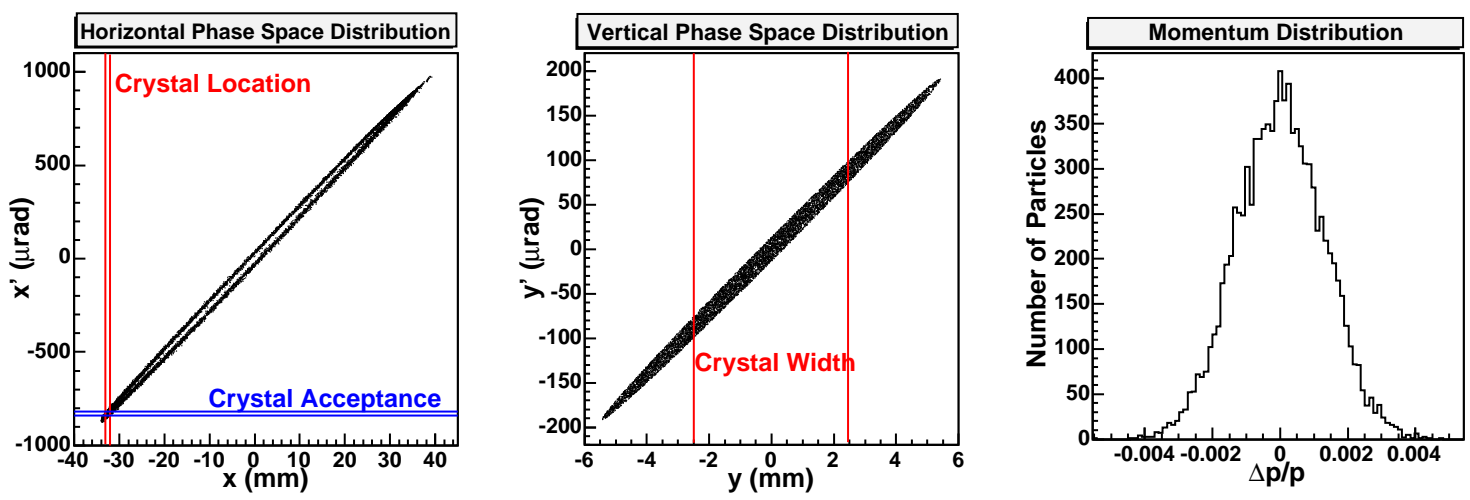

Figure 4.5: Typical input distribution for crystal simulations. The red lines show the crystal edges, while the blue lines show the crystal angular acceptance centered on $-830 \mu \mathrm{rad}$. 
are then read into a ROOT class designed for the analysis of these simulations.

\subsubsection{CORAANT}

The ROOT framework contains a plethora of general purpose features, but is geared for high energy nuclear and particle physics applications. The author saw the necessity to start to write special purpose analysis code for accelerator physics

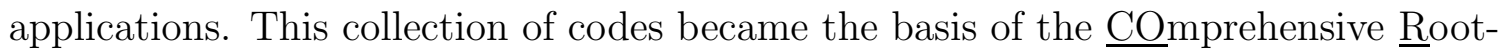

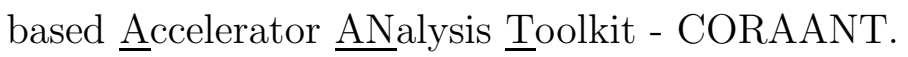

Features of CORAANT include the ability to make graphs from ROOT TNtuple's, TTree's, and TChain's via CORAANT's TGNtuple, TGTree, and TGChain classes. CORAANT uses the FFTW package [69] to do fast Fourier transforms so that tune diagrams can be drawn. Beam position monitor data can also be analyzed this way. CORAANT will continue to develop with time as the number of its users and their needs increase.

\subsection{Crystal Channeling}

The upstream PIN diodes and the crystal hodoscope observe a fraction of the particles that scatter from the crystal. Particles that channel are lost from the scattering signal, and result in a reduction of the scattering rate in the upstream PIN diodes and hodoscope. Figure 4.6 shows a scan simulation of the number of particles scattered from a crystal verses the crystal angle. This is called a crystal scan. The large thin dip occurs at an angle, $\theta=830 \mu \mathrm{rad}$ where the crystal planes are aligned to the incoming particles as predicted by Equation 2.72

$$
x_{p}^{\prime}=\left(x_{0}+\frac{\Delta x}{2}\right) \frac{\sigma_{x x^{\prime}}}{\sigma_{x}^{2}} .
$$




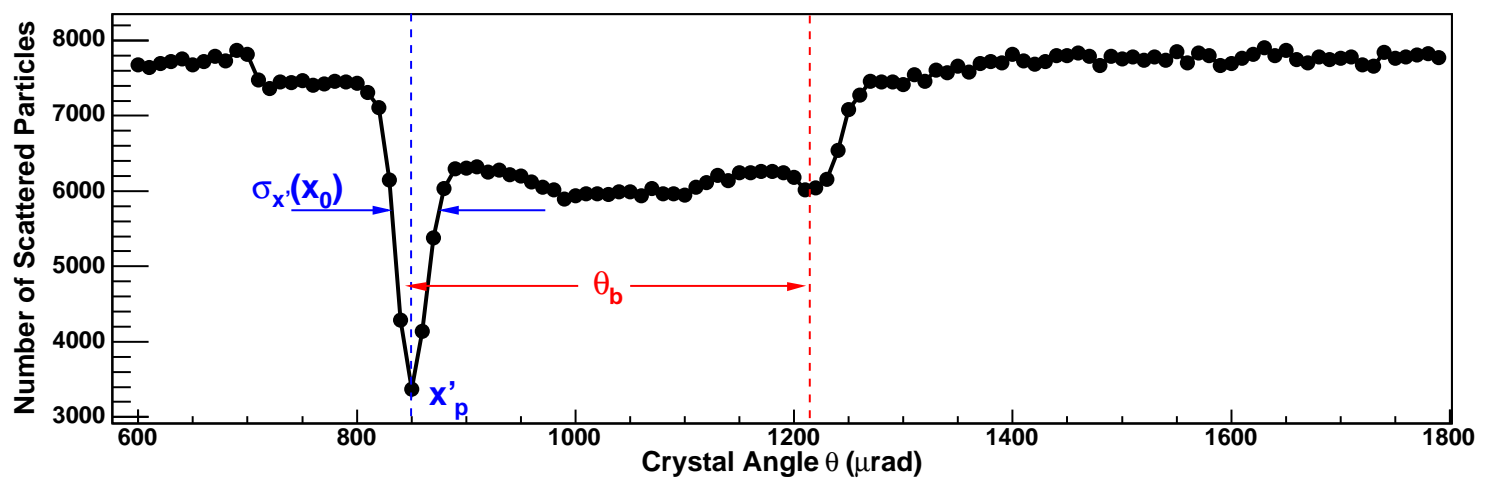

Figure 4.6: Simulation of the number of scattered particles as the crystal angle is changed. The reduction in the scattering rate indicates channeling. The crystal is properly aligned at $\theta=830 \mu \mathrm{rad}$.

The width of the dip, predicted by Equation 2.74,

$$
\begin{aligned}
\sigma_{x^{\prime}}\left(x_{0}\right)= & \frac{\sqrt{\epsilon\left(\epsilon+\langle\mathcal{H}\rangle \sigma_{p}^{2}\right)}}{\sigma_{x}}+\frac{\sigma_{x x^{\prime}}^{2} \Delta x^{2}}{24 \sigma_{x}^{3} \sqrt{\epsilon\left(\epsilon+\langle\mathcal{H}\rangle \sigma_{p}^{2}\right)}} \\
& \left\{1-\frac{\Delta x^{2}}{240 \sigma_{x}^{2}}\left[8+5 \frac{-\alpha \epsilon \sigma_{x x^{\prime}}+D^{2} D^{\prime 2}{\sigma_{p}}^{4}}{\epsilon\left(\epsilon+\langle\mathcal{H}\rangle \sigma_{p}^{2}\right)}+12 \frac{x_{0}^{2}}{\sigma_{x}^{2}}\right]-\frac{x_{0} \Delta x^{3}}{20 \sigma_{x}{ }^{4}}\right\}+O\left(\Delta x^{6}\right)
\end{aligned}
$$

is wider than expected. The reason for this is discussed below. Volume capture occurs when the crystal angle is between $900 \mu \mathrm{rad}$ and $1200 \mu \mathrm{rad}$. At these angles, the particles are not aligned to the planes upon entering the crystal, but can scatter into the planes after traversing some distance in the crystal. The channeling stops at $\theta \approx 1275 \mu \mathrm{rad}$ because the planes are rotated too far for the particles to scatter into them.

From Figure 4.6, an estimate of the channeling efficiency is made by taking the ratio of reduction in scattering of the large dip to the scattering rate of particles with no channeling. The estimated efficiency for Figure 4.6 is $56 \pm 1 \%$, compared to the $52 \%$ expected from Equation 2.29.

The number of turns tracked has an effect on the width of the large dip and the 


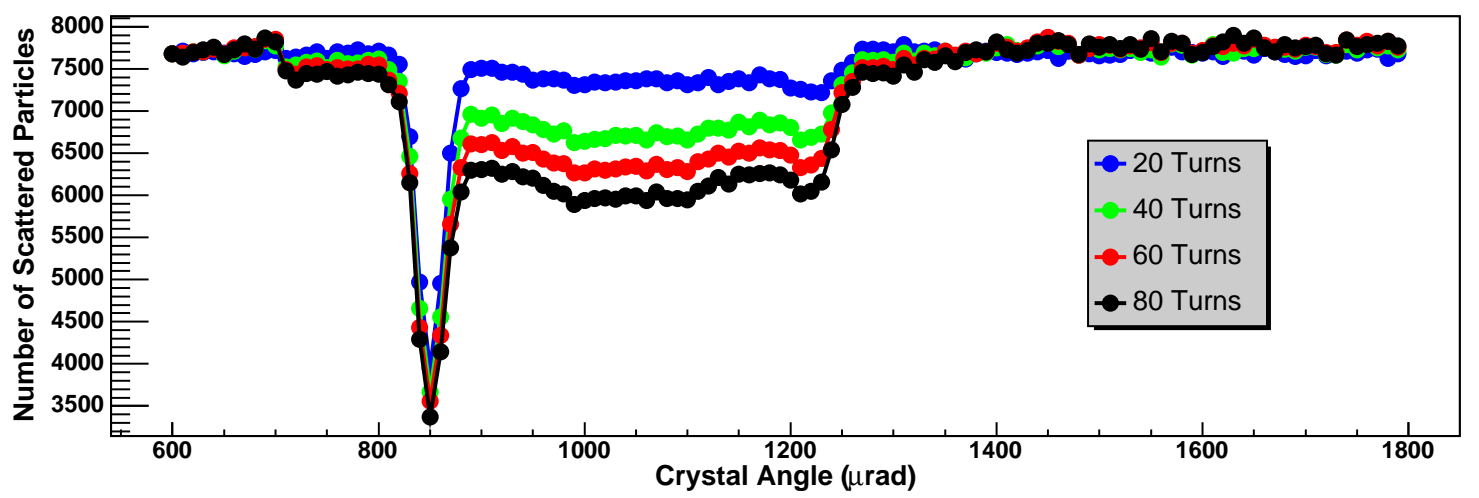

Figure 4.7: Simulation of the effect of multiple turns on the number of particles scattered from the crystal. Particles that scatter through the crystal on one pass change their incident angle on the next pass through the crystal, increasing the width of the large dip and the efficiency of the volume capture.

efficiency of volume capture. Figure 4.7 shows the effect. This is because particles that scatter through the crystal on one pass change their incident angle on another pass through the crystal. This is covered extensively in Chapter 4.2 of Reference [9].

The effect of optical errors at the crystal was simulated by varying the Twiss parameters at the beginning and end of the collimator section. Figure 4.8 shows the effect of varying $\alpha$ at the crystal while keeping the remaining Twiss parameters constant.

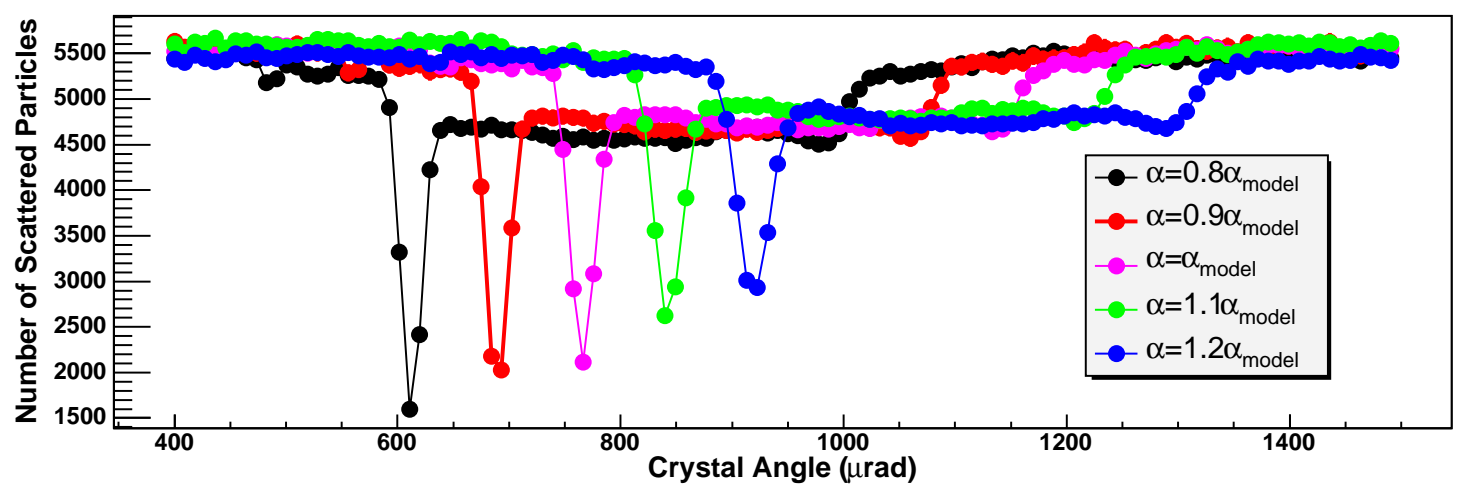

Figure 4.8: Simulation of the effect of changing $\alpha$ on channeling. Errors in Twiss parameters result in changes in the channeling efficiency. 

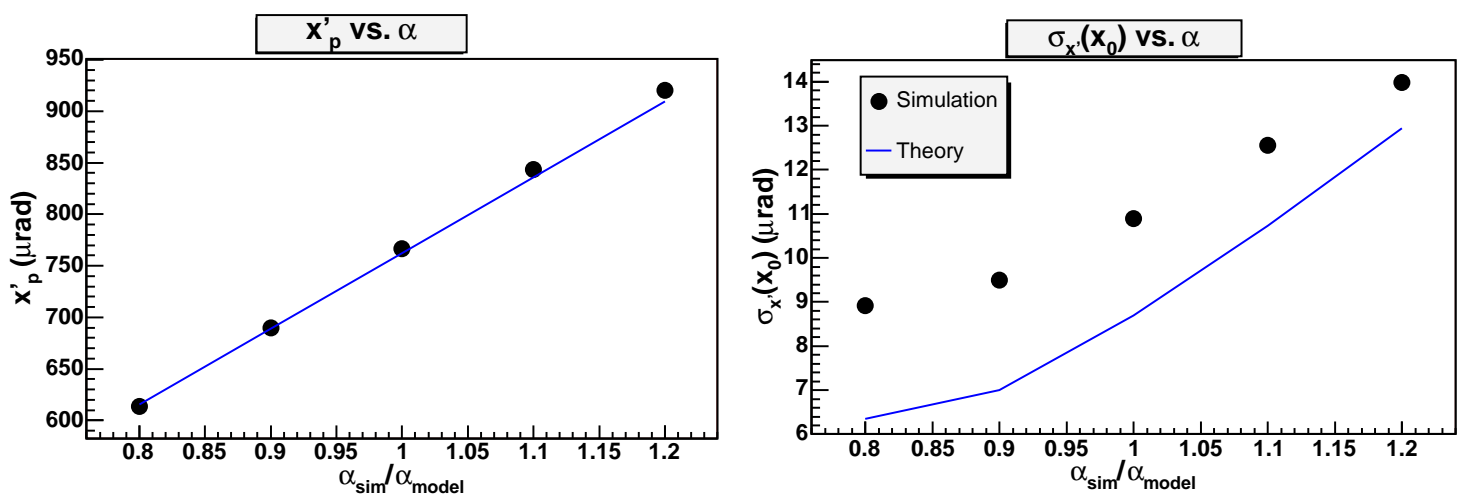

Figure 4.9: Comparison of simulations in Figure 4.8 to theory. The left figure illustrates the agreement between the predicted and simulated location of channeling dip, $x_{p}^{\prime}$ as a function of $\alpha$. The right figure shows the disagreement between the predicted and simulated width, $\sigma_{x^{\prime}}\left(x_{0}\right)$ as a function of $\alpha$.

Figure 4.9 compares the simulations in Figure 4.8 to Equations 2.72 and 2.74 . The simulation of the crystal scans agrees with Equation 2.72 quite well although the slope is $4 \%$ greater than the theory. The width of the channeling dip, $\sigma_{x^{\prime}}\left(x_{0}\right)$ predicted by theory is smaller than the simulations show. Equation 2.74 does not take into account the effect of particles scattering through the crystal and returning on subsequent turns. This broadens the width of the dip. One turn simulations agree with Equation 2.74 as Figure 4.10 shows.

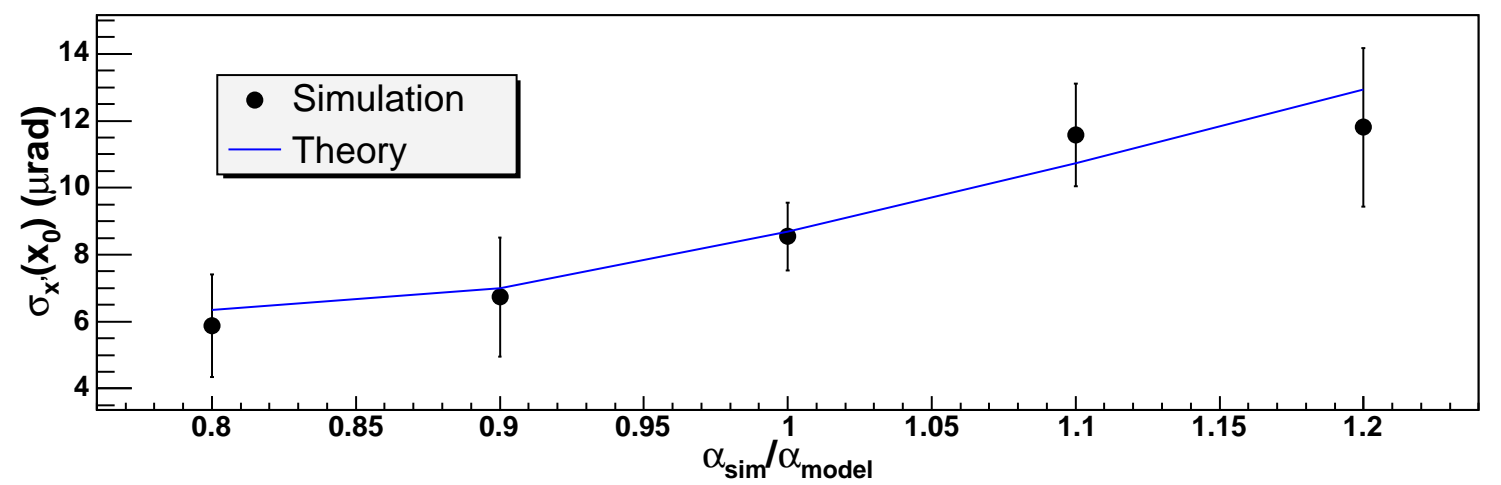

Figure 4.10: Comparison of predicted and simulated width, $\sigma_{x^{\prime}}\left(x_{0}\right)$, as a function of $\alpha$ for one turn simulations. 


\subsection{Collimation Efficiency}

Another important quantity to simulate is the distribution of lost particles. A PERL script, named raystrack, was written to simulate this. Raystrack uses UAL to propagate particles around the accelerator, K2 to propagate particles in the scraper, and CATCH to propagate particle through the crystal. Raystrack tracks particles around the ring element by element recording particles that exceed the local aperture. The start and finish coordinate of the particles are written to file. The location of the loss is also recorded for every particle that is lost. A map of losses can be reconstructed from the simulation in this way.

This type of simulation requires knowledge of the machine aperture at all locations. Unfortunately, only the apertures for magnets were known before the FY2004 run. It is possible to fully reproduce aperture of RHIC in simulation for the FY2004 run. The technical details of implementing the aperture model is discussed in Appendix A. Figure 4.11 shows the results of a simulation done in the blue ring with $\beta^{*}=2 \mathrm{~m}$ at all interaction regions.

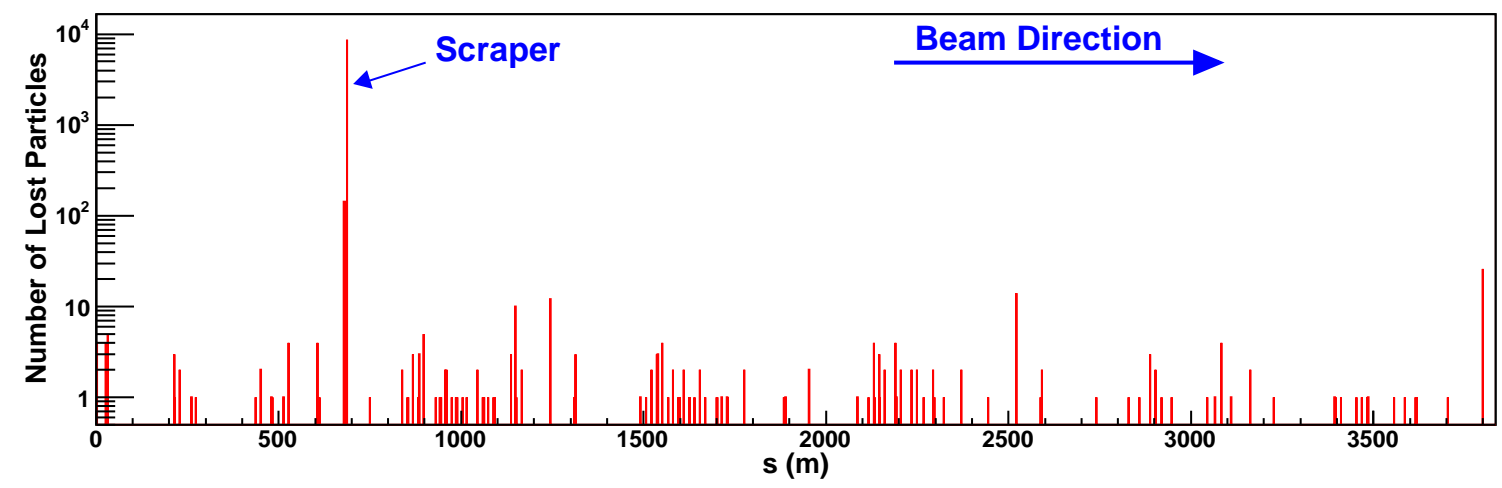

Figure 4.11: Simulation of locations of particles lost in RHIC Blue Ring after hitting scraper.

Unfortunately, UAL did not have a way of keeping a record of the locations of particle losses, so it was necessary to loop over the particles after each element to 
check if any had been lost. This significantly slowed down the simulation for large number of particles and elements. A new class was added to TEAPOT called LostCollector that automatically records this information when particles are tracked through an element. After the simulation, the LostCollector saves the element location, name, turn number and phase space coordinates of all of the lost particles to an ASCII file for later analysis.

\subsection{Comparison of ACCSIM and K2}

Two codes, ACCSIM and K2, are proposed for use in future RHIC collimation simulations. Therefore it is important to understand any differences between them. To understand this, the output of both codes is compared for the same input distribution. A simple, flat particle distribution in both transverse phase spaces and momentum spread is chosen. This distribution is shown in Figure 4.12. This same particle distribution is input into both codes for a horizontal copper collimator. The collimator is chosen to slice the horizontal phase space in half. In this way, the handling of scattering out of the edge and drift spaces is tested, as well as the interactions in the material.

It is found that ACCSIM and K2 give very similar angular and energy distributions after scattering through the collimator. However, K2 produces larger tails in these distributions. Figure 4.13 shows the angular distribution and energy loss of particles that scatter inelastically in the collimator. The number of particles that are lost in ACCSIM is 48766, and in $\mathrm{K} 2$ is 48538. The histograms are cut to remove the tails of the distributions of $\mathrm{K} 2$ which extend out to $\pm 10 \mathrm{mrad}$ in the angles and to $\delta=-0.19$ in momentum offset. The angular histograms are missing $0.10 \%$ of the K2 distribution because of the cut. The energy loss histogram is missing $3.3 \%$ of the 

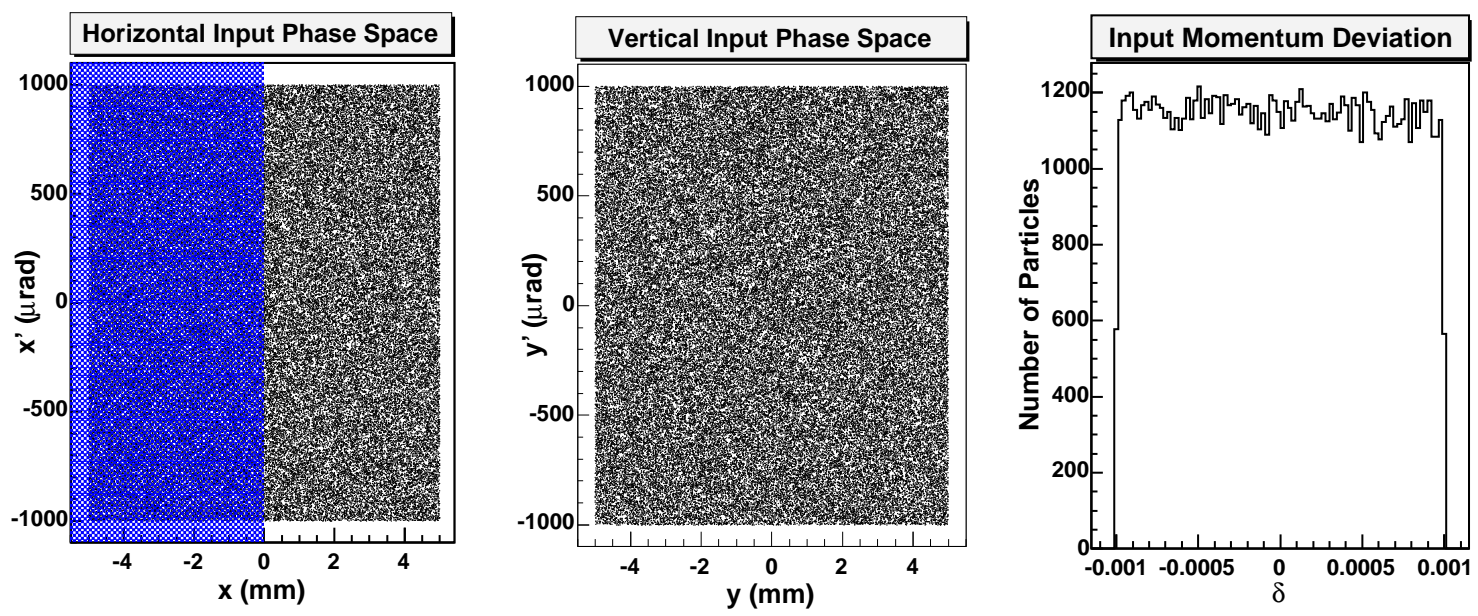

Figure 4.12: Particle distribution used to compare ACCSIM and K2. Horizontal and vertical phase space and momentum deviation are shown. The shaded area denotes the collimator area. There are 100,000 particles.

particles from K2.

The energy loss is the largest difference between the two codes. Both codes calculate the total energy loss as a mean energy loss per unit length times the length traveled in the collimator. ACCSIM calculates the mean energy loss per unit length using the Bethe-Bloch formula (Equation 2.31), while K2 uses the energy loss of
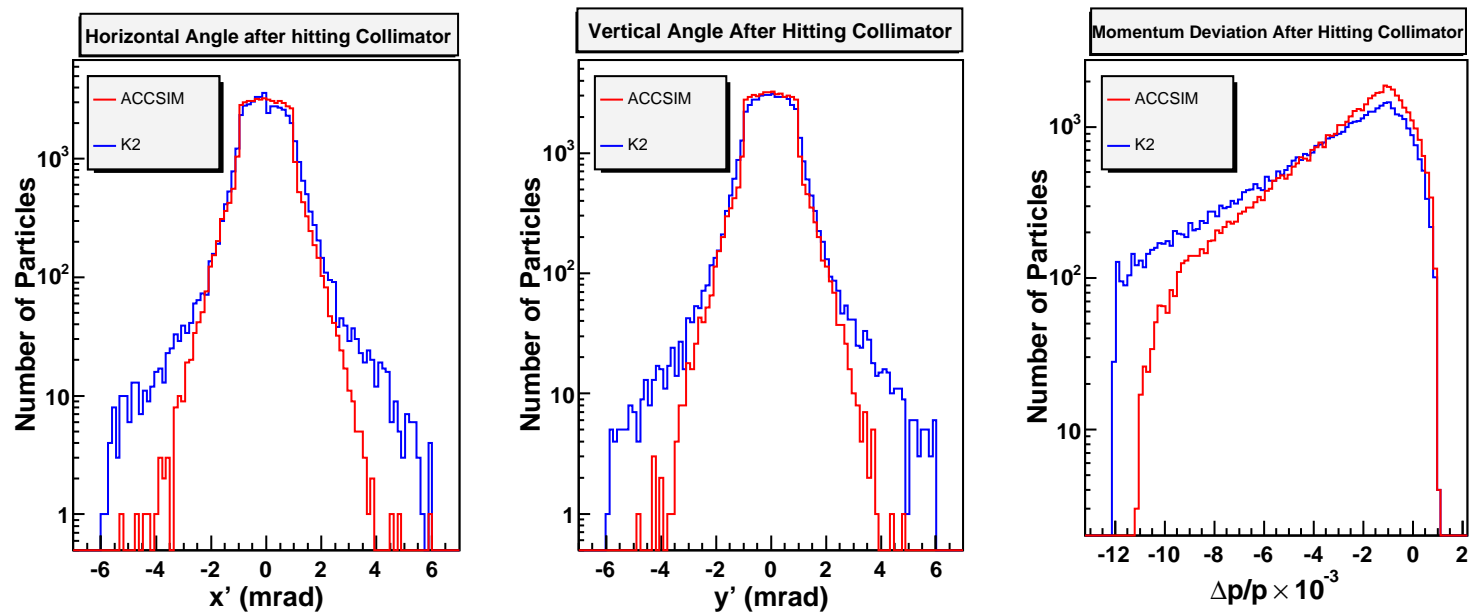

Figure 4.13: The effects of scattering in K2 and ACCSIM. K2 produces larger tails in all cases. 
a proton traveling through the selected material with an energy of $450 \mathrm{GeV}$, the injection energy of the Large Hadron Collider. This is approximately a factor of 1.3 larger than a $100 \mathrm{GeV}$ proton traveling in the same material. Each code handles multiple Coulomb scattering in a similar way. However, there are corrections to the scattering angle for diffractive scattering in $\mathrm{K} 2$.
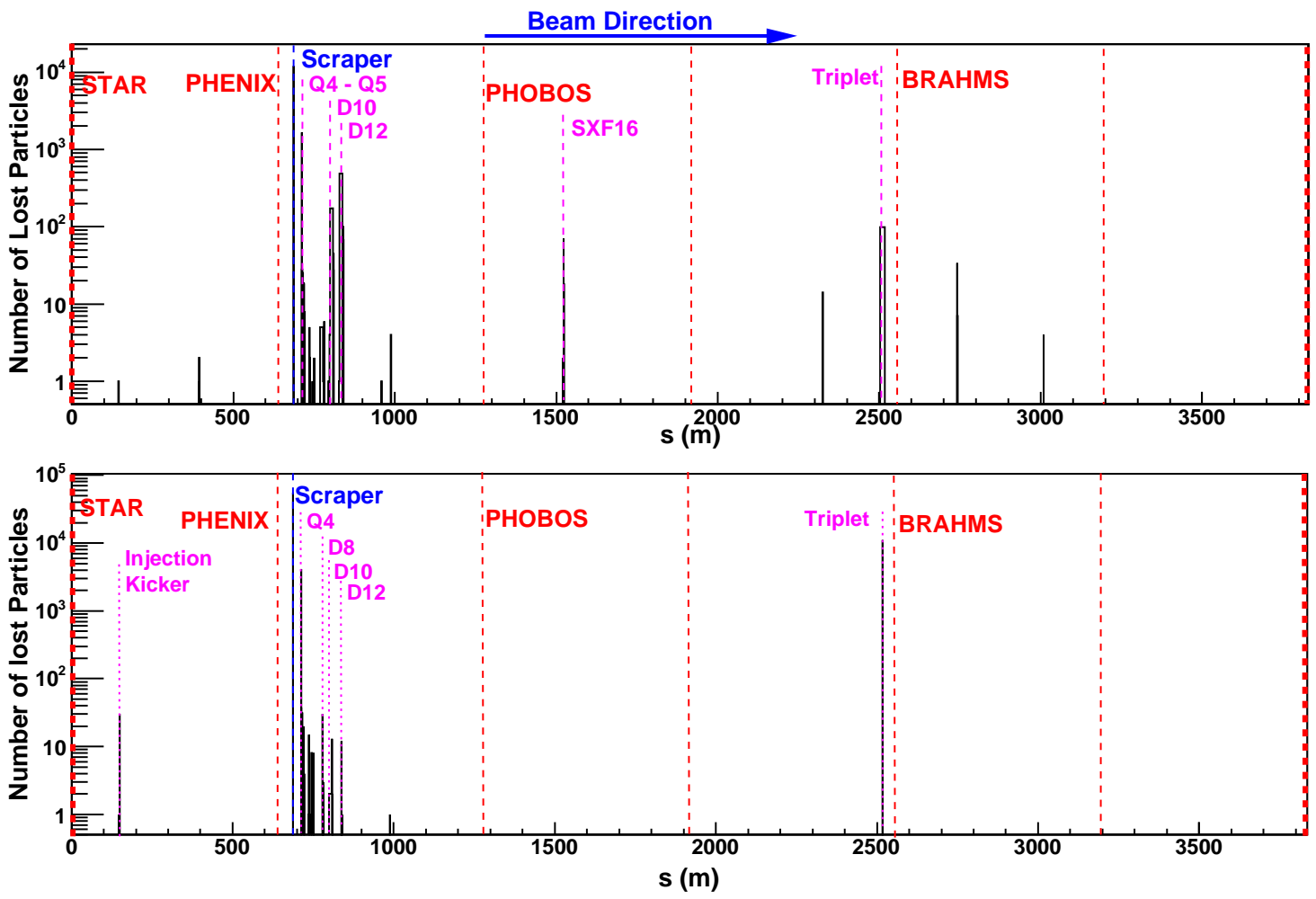

Figure 4.14: Comparison of Loss Location simulations between ACCSIM (top) and K2 (bottom). Loss locations are similar. K2 loss locations are localized to the 8-9 o'clock arc. ACCSIM losses are more distributed. The vertical axes have a log scale.

Figure 4.14 shows a comparison of beam loss locations using ACCSIM and K2. Each simulation uses a beam distribution identical to the one discussed in Section 4.1.5, though it is not the identical distribution in each simulation. Each simulation contains $10^{5}$ protons, and are tracked for 20 turns through the FY2003 deuteron-gold injection lattice. The loss pattern is similar in many respects. There are large losses at quadrupole 4 (Q4), and dipoles 8, 10, and 12 (D8, D10, D12), and the incoming 
BRAHMS triplet. The pattern of the losses differ among those elements. The K2 distribution quickly falls through D12, whereas the ACCSIM distribution falls then grows at D10 and D12. More particles are lost in the scraper and the BRAHMS triplet in the K2 simulation. The ACCSIM simulation has more loss locations.

The similarity of the horizontal and vertical angle outputs of the codes is probably not the cause of the difference. The energy loss is most likely the source of the difference. Even a small dispersion will cause particles to be lost sooner if they acquire larger momentum offsets in the scraper. Particles will smaller momentum offsets will travel farther to large dispersion areas, such as dipoles. A comparison of these simulations using ACCSIM to data occur in Section 6.3. More work needs to be done to benchmark K2 and ACCSIM with data and understand the difference between them.

These codes provide the necessary flexibility and tools to properly simulate the RHIC collimation system. It is possible to simulate collimator performance under a variety of conditions to predict the overall efficiency of the system. Chapters 5 and 6 contain comparisons between the data and the results of these simulations. 


\section{Chapter 5}

\section{Crystal Channeling Measurements}

This chapter details the measurements made with the crystal collimator during the FY2001 through FY2003 RHIC runs. Measurements using only the scraper are covered in the following chapter. During the FY2001 gold run and FY2002 polarized proton run efforts focused primarily on commissioning the crystal collimator and measuring its channeling properties. Our goal for the FY2003 deuteron - gold run was to measure and understand the performance of the crystal collimator as the first stage in a two stage collimation system.

Most of these data were taken during routine operations with minimal interference to the RHIC detectors. A few unsuccessful studies were performed at injection energy for comparison purposes. The injection studies were unsuccessful because of the beam position oscillations discussed in Section 6.6. Table 5.1 lists the existing data sets. The term "scan" indicates that the crystal collimator was inserted to a particular transverse position and then rotated through a range of angles with respect to the beam orbit. During each scan, the beam loss rate was measured by the available detectors. The experimental background rates were also logged for a large number of the scans. 
Table 5.1: Synopsis of Channeling Data

\begin{tabular}{|c|c|c|c|c|}
\hline Run & Species & $\beta_{P H E N I X}^{*}$ & No. of Stores & No. of Scans \\
\hline FY2001 & $\mathrm{Au}$ & $5 \mathrm{~m}$ & 8 & 27 \\
FY2001 & $\mathrm{Au}$ & $2 \mathrm{~m}$ & 4 & 24 \\
FY2001 & $\mathrm{Au}$ & $1 \mathrm{~m}$ & 12 & 109 \\
FY2002 & $\mathrm{p}$ & $3 \mathrm{~m}$ & 11 & 119 \\
FY2003 & $\mathrm{Au}$ & $2 \mathrm{~m}$ & 4 & 20 \\
\hline
\end{tabular}

For the FY2003 run, the crystal was changed to another crystal of identical design and lattice orientation. This was done so that the first crystal could be examined for defects (cracks, surface scratches, wrong lattice orientation, etc.). Each crystal was examined by X-Ray diffraction and was determined to be of very good quality with the correct lattice orientation [70]. It was not possible to determine the miscut angle of the crystal from this measurement because the bend angle of the crystal is large enough to distort the lattice to make determination of the miscut angle impossible without accurate knowledge of the bend angle.

\subsection{Data Acquisition and Analysis}

In order to measure the channeling characteristics of the crystal collimator, the crystal collimator is inserted into the beam halo, and its angle with respect to the beam orbit is scanned. Figure 5.1 is an example of a typical crystal scan using one of the upstream PIN diodes to monitor scattering from the crystal. In this scan, the crystal is $33 \mathrm{~mm} \approx 6 \sigma_{x}$ from the beam. The angle of the crystal is moved in steps from left to right in the figure. The size of the step and the dwell time at each step is controllable. The PIN diodes are read out at $1 \mathrm{~Hz}$. The drop in the scattering rate indicates channeling in the crystal.

The vertical error bars are statistical. The horizontal error bars are given by the resolution of the angular readback, approximately $25 \mu \mathrm{rad}$. The "crystal angle" 


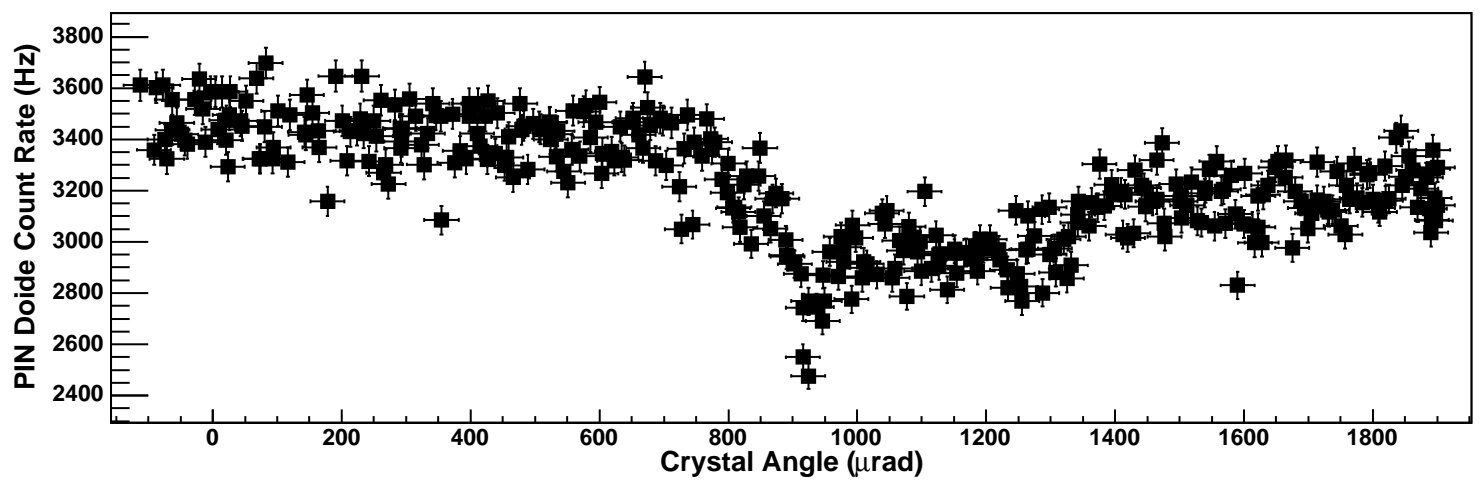

Figure 5.1: Typical Crystal Scan. The reduction in the scattering rate indicates crystal channeling. Data is from Fill 01737.

plotted is the angle between the crystal and the beam orbit as measured by the BPMs and is shown in Figure 2.4.

All of the data were taken once RHIC was fully optimized for luminosity production so that conditions were stable. The data taken in the FY2003 run had the copper scraper inserted into the beam. This was necessary to reduce beam backgrounds in all of the experiments.

Once the data were logged, they were converted into ROOT files for analysis. Each scan was divided into $20 \mu$ rad bins. A weighted average was computed for each bin to reduce fluctuations in the data. The averaged data were fit to:

$$
f(\theta)=\left\{\begin{array}{cl}
A_{1}\left[1+\frac{\left(\theta-\theta_{1}\right)^{2}}{8 \ln 2 w_{1}^{2}}\right]^{-1}+S \theta+T \quad: \quad \theta \leq \theta_{A} \\
A_{2}+S \theta+T \quad: \quad \theta_{A}<\theta<\theta_{2} \\
A_{2}\left[1+\frac{\left(\theta-\theta_{2}\right)^{2}}{8 \ln 2 w_{2}^{2}}\right]^{-1}+S \theta+T \quad: \quad \theta_{2} \leq \theta
\end{array}\right.
$$

where $\theta_{1,2}, w_{1,2}$, and $A_{1,2}$ are the centers, widths, and amplitudes of the left and right lorentzian dips, $\theta_{A}$ is the end of the left lorentzian, and $S$ and $T$ are the slope and offset of the background. The value of $\theta_{A}$ is determined by continuity of $f\left(\theta=\theta_{A}\right)$. Lorentzian distributions were chosen for fitting because initial fits with 
gaussian distributions showed that the data contained non gaussian tails. Lorentzian distributions more closely matched the data.

It should be noted that $w_{1,2}$ are not the lorentzian full widths at half maximum (FWHM) as is typically used for a lorentzian distribution [71]. Instead they are the rms of a gaussian distribution with the equivalent FWHM. We did this to compare the width from these fits to Equation 2.74 where a gaussian distribution is used. Figure 5.2 shows the data from Figure 5.1 after averaging. The fit is shown as a solid red line.

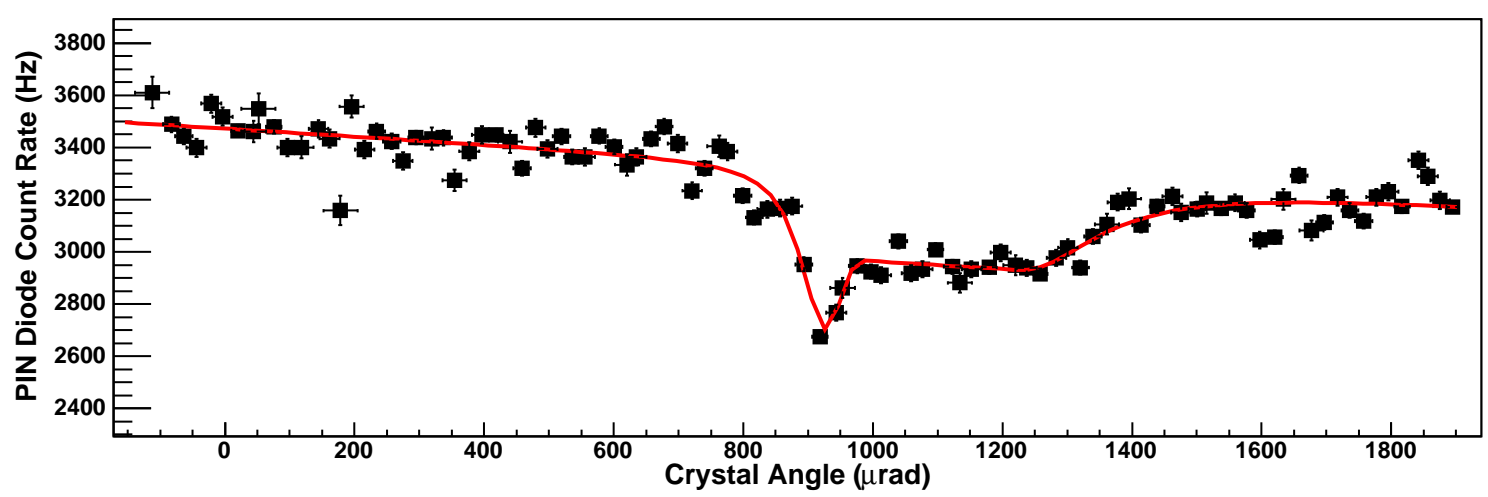

Figure 5.2: Data from Figure 5.1 after averaging. The red line corresponds to the fit to Equation 5.1.

Each of the hodoscope scintillators and their coincidence signals were analyzed in the same fashion. The analyzed data from Fill 01547 are shown in Figure 5.3. However, the scintillators are subject to backgrounds coming from particles scraping in the interaction region triplet magnets immediately upstream of them. The signal from Scintillator 2, is reduced because of its distance from the crystal. The coincidence signal has a reduced background, but suffers from the loss of signal in Scintillator 2. After the FY2002 run, the voltages on the photomultiplier tubes and discriminator thresholds were adjusted in an attempt to maximize the hodoscope signals. Particular attention was paid to Scintillator 2. However, the hodoscope per- 

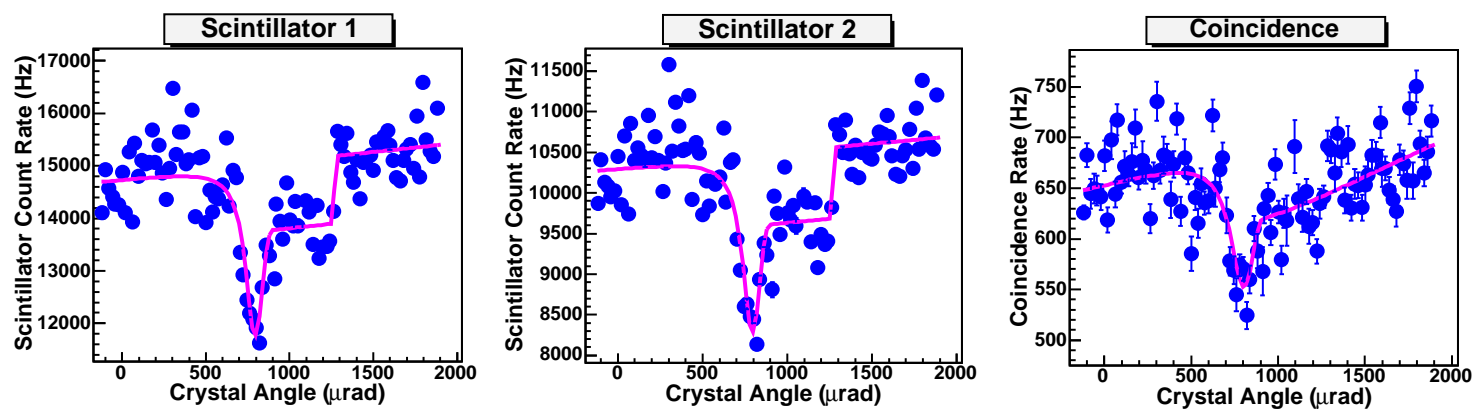

Figure 5.3: Hodoscope Data during Fill 01547. The statistical error bars on the left and center graphs are smaller than the point size.

formance did not improve for the FY2003 run. The reasons for this are not completely understood, although poor gain in the photomultiplier tube is suspected.

The data were divided into good and bad data sets. A set was declared bad based on the following criteria:

- incomplete scans due to technical problems

- scans with low signal

- the beam has position oscillations due to the AGS Booster cycle (Section 6.6)

- scans that are well outside of the angular acceptance of the crystal

Of the 301 total scans, approximately $40 \%$ of the scans are declared good.

The downstream PIN diodes are sensitive to scattering from both the crystal collimator and the scraper. Depending on the relative locations of the crystal and scraper to each other, these PIN diodes may detect an increase in signal rate when the crystal is channeling and the channeled beam is striking the scraper. This case is shown in Figure 5.4. If the scraper is retracted, these PIN diodes see a reduction in scattering when the crystal is channeling, similar to the upstream PIN diodes. This is because no beam strikes the scraper at all. Should the scraper be in an intermediate 


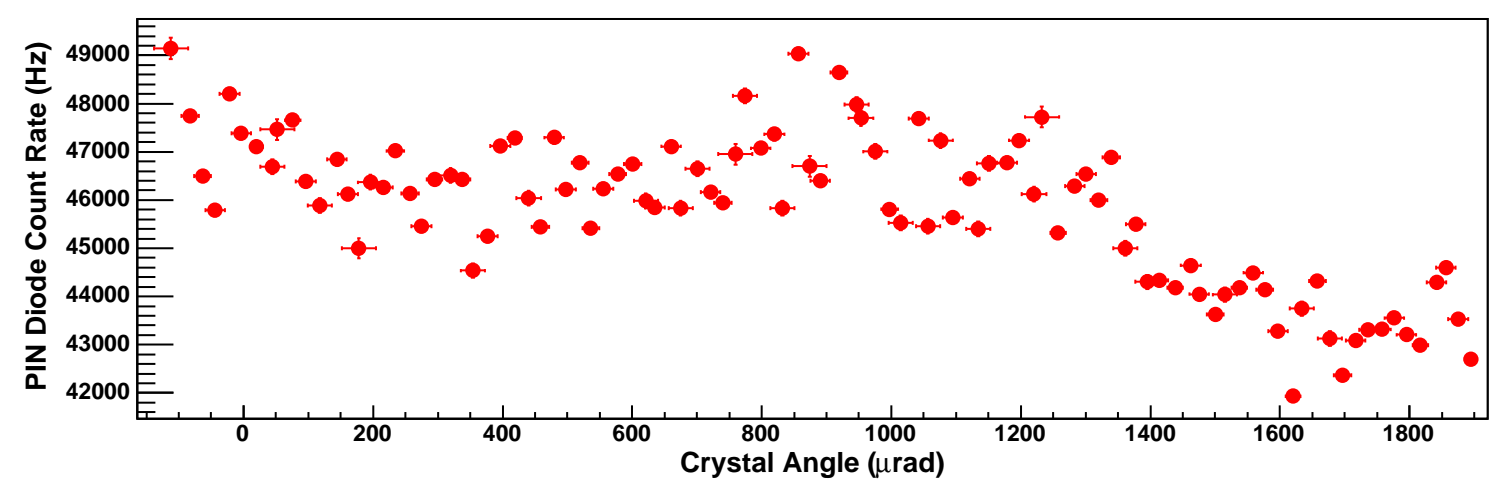

Figure 5.4: Averaged downstream PIN diode rate during an angular scan

location, they may see an increase or decrease in scattering depending on whether or not the channeled beam is hitting the scraper.

In all cases it is difficult to meaningfully fit these downstream PIN diode data as Figure 5.4 shows. However, the downstream PIN diode data are useful in determining if the channeled beam is striking the scraper. If there are high backgrounds from other local losses such as the scraper being used for vertical collimation, it is possible that the channeled beam is not seen, even though it is striking the scraper.

\subsection{Channeling Characteristics}

The initial focus was to understand the channeling characteristics of the crystal collimator, such as the channeling efficiency, in order to understand how effective crystal collimation would be. This was the focus during the FY2001 and FY2002 runs.

Figure 5.5 shows a comparison between two simulations and data from the FY2001 run. The blue curve shows the simulation with the design optics and the red curve is the simulation with the measured optics. There are 40 turns in each simulation. Each simulation is scaled vertically to match the number of scattered 


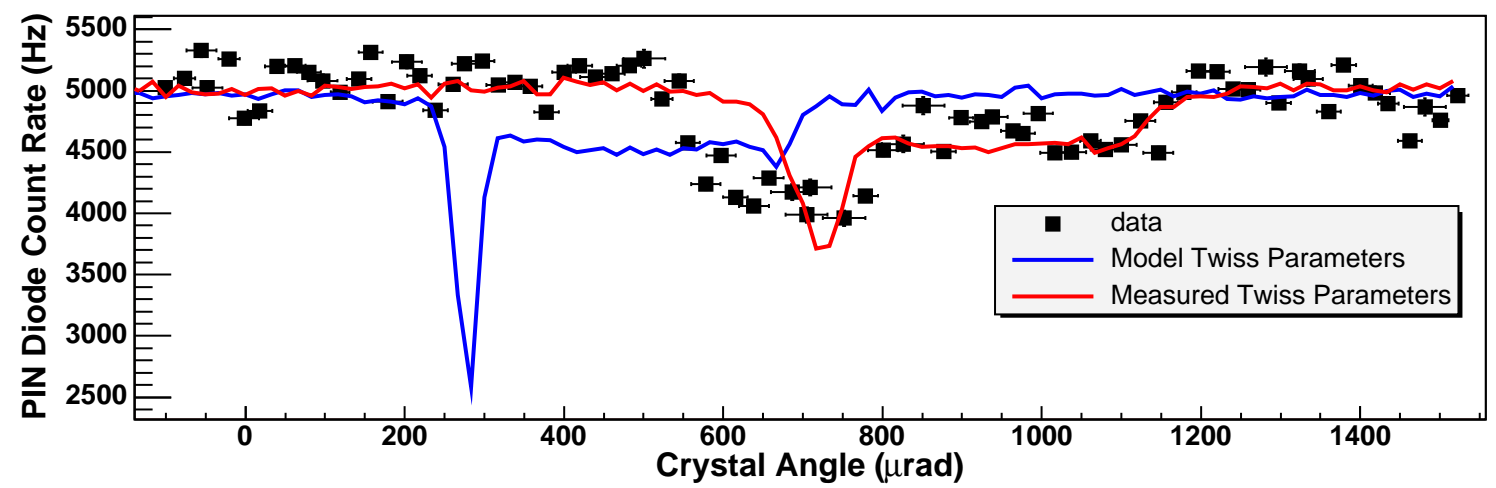

Figure 5.5: Comparison of simulations with the data from Fill 1547. The distance between the crystal and beam is $29.0 \mathrm{~mm}$.

particles to the data background. Each simulation is shifted horizontally to take into account the miscut angle and the angular offset of the crystal. The shift is chosen to match the large dip in the measured optics simulation to the data, and is the same for each simulation.

The simulation using the design optics reproduces the general shape of the data. However, the position of the channeling peak is offset, reflecting the large change in $\sigma_{x x^{\prime}} / \sigma_{x}^{2}$ between the design and measured optics. The width is 5 times narrower than the data show. The efficiency, $\epsilon$, defined as the depth of the left dip to the background rate, is approximately 2.6 times larger than the channeling data. The measured Twiss parameters show better agreement with the channeling data. The simulated efficiency using the measured Twiss parameters is 1.5 times larger and the width is 3.2 times smaller than the data.

Table 5.2 compares the width and efficiency of simulations using the design and measured optics to channeling data for selected fills for each $\beta_{P H E N I X}^{*}$. All simulations using the design optics predict channeling efficiencies greater than two times the measured value. In two of the cases, the predicted efficiency using the available measured optics is less than the efficiency predicted with the design optics 
Table 5.2: Comparison of selected data sets to simulations

\begin{tabular}{|c|c|c|c|c|c|c|c|}
\hline Run & Fill & $\beta^{*}[\mathrm{~m}]$ & $x_{0}[\mathrm{~mm}]$ & $\begin{array}{c}\text { Determined } \\
\text { from }\end{array}$ & Turns & $\sigma_{x^{\prime}}\left(x_{0}\right)[\mu \mathrm{rad}]$ & $\epsilon[\%]$ \\
\hline \multirow{3}{*}{ FY2001 } & \multirow{3}{*}{01118} & \multirow{3}{*}{5} & \multirow{3}{*}{-34.9} & $\begin{array}{l}\text { Design } \\
\text { Optics }\end{array}$ & 60 & $19.4 \pm 0.4$ & $51.0 \pm 0.6$ \\
\hline & & & & $\begin{array}{c}\text { Measured } \\
\text { Optics }\end{array}$ & - & - & - \\
\hline & & & & $\begin{array}{c}\text { Channeling } \\
\text { Data } \\
\end{array}$ & - & $63 \pm 10$ & $22 \pm 2$ \\
\hline \multirow{3}{*}{ FY2001 } & \multirow{3}{*}{01547} & \multirow{3}{*}{2} & \multirow{3}{*}{-29.0} & $\begin{array}{l}\text { Design } \\
\text { Optics }\end{array}$ & 20 & $12 \pm 1$ & $55 \pm 2$ \\
\hline & & & & $\begin{array}{c}\text { Measured } \\
\text { Optics }\end{array}$ & 20 & $20 \pm 1$ & $37 \pm 1$ \\
\hline & & & & $\begin{array}{c}\text { Channeling } \\
\text { Data }\end{array}$ & - & $64 \pm 4$ & $22 \pm 1$ \\
\hline \multirow{3}{*}{ FY2001 } & \multirow{3}{*}{01737} & \multirow{3}{*}{1} & \multirow{3}{*}{-32.5} & $\begin{array}{l}\text { Design } \\
\text { Optics }\end{array}$ & 40 & $13 \pm 1$ & $54 \pm 2$ \\
\hline & & & & $\begin{array}{c}\text { Measured } \\
\text { Optics }\end{array}$ & 40 & $11 \pm 1$ & $56 \pm 3$ \\
\hline & & & & $\begin{array}{c}\text { Channeling } \\
\text { Data }\end{array}$ & - & $33 \pm 1$ & $22 \pm 1$ \\
\hline \multirow{3}{*}{ FY2002 } & \multirow{3}{*}{02095} & \multirow{3}{*}{3} & \multirow{3}{*}{-29.8} & $\begin{array}{l}\text { Design } \\
\text { Optics }\end{array}$ & 80 & $22.6 \pm 0.3$ & $63.4 \pm 0.5$ \\
\hline & & & & $\begin{array}{c}\text { Measured } \\
\text { Optics }\end{array}$ & - & - & - \\
\hline & & & & $\begin{array}{c}\text { Channeling } \\
\text { Data }\end{array}$ & - & $49 \pm 2$ & $32 \pm 1$ \\
\hline \multirow{3}{*}{ FY2003 } & \multirow{3}{*}{03061} & \multirow{3}{*}{2} & \multirow{3}{*}{-17.6} & $\begin{array}{l}\text { Design } \\
\text { Optics }\end{array}$ & 20 & $10.8 \pm 0.4$ & $61 \pm 2$ \\
\hline & & & & $\begin{array}{c}\text { Measured } \\
\text { Optics }\end{array}$ & 20 & $16 \pm 1$ & $51 \pm 1$ \\
\hline & & & & $\begin{array}{c}\text { Channeling } \\
\text { Data }\end{array}$ & - & $51 \pm 1$ & $30.0 \pm 0.4$ \\
\hline
\end{tabular}

$\beta^{*}$ denotes the $\beta^{*}$ in the PHENIX detector. $x_{0}$ is the distance between the crystal and beam center, and is the same for both the simulation and the particular dataset. The determined from column indicates the optics used in the simulation or if the values from the fit to channeling data are used. The number of turns is chosen to best fit the dataset.

but is still 1.7 times too large. Differences between the simulations and the channeling data are discussed later in this section.

During the $\beta_{P H E N I X}^{*}=5 \mathrm{~m}$ run, the Twiss parameters were not measured. 
The data from a number of the BPMs in the vicinity of the collimation system were corrupted for part of the FY2002 run. Unfortunately it was during this time that the optics measurements were taken, so the Twiss parameters at the crystal are unknown. For the $\beta_{P H E N I X}^{*}=5 \mathrm{~m}$ and $\beta_{P H E N I X}^{*}=3 \mathrm{~m}$ runs, a comparison with the measured optics is impossible.

The $\beta$ and $\alpha$ functions for the $\beta_{P H E N I X}^{*}=1 \mathrm{~m}$ optics were interpolated to the crystal because the BPM immediately downstream of the collimator section malfunctioned during the $\beta$ function measurements. Another BPM was used instead. There was a quadrupole between this BPM and the crystal. The measured Twiss parameters are the same as design within the error of the measurement [72].

Measuring the channeling angle, $x_{p}^{\prime}$, as a function of the distance between the crystal and the beam, $x_{0}$, is a way to measure $\sigma_{x x^{\prime}} / \sigma_{x}^{2}$. This is shown in Figure 5.6 for all of the $\beta_{P H E N I X}^{*}=1 \mathrm{~m}$ data. The data are fit to a line whose slope is given by $\sigma_{x x^{\prime}} / \sigma_{x}^{2}$ as in Equation 2.72 .

$$
x_{p}^{\prime}=\left(x_{0}+\frac{\Delta x}{2}\right) \frac{\sigma_{x x^{\prime}}}{\sigma_{x}^{2}}
$$

Points with $x_{0}>37 \mathrm{~mm}$ have lower angles than predicted by the model. At

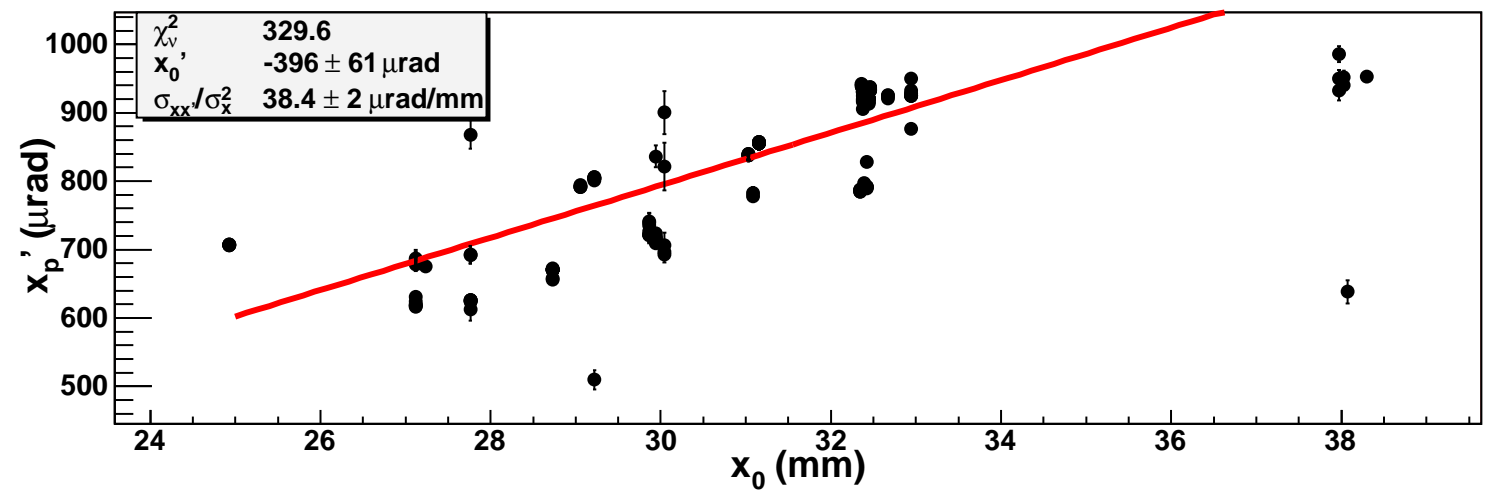

Figure 5.6: Graph of the channeling dip, $x_{p}^{\prime}$, vs. crystal location, $x_{o}$ for $\beta_{P H E N I X}^{*}=$ $1 \mathrm{~m}$. All points are included in the fit. 
positions in this range, the crystal is very much in the beam halo and the signal to noise ratio is small. Nevertheless, the points are included in the fit. Removing them from the fit increases the fit value of $\sigma_{x x^{\prime}} / \sigma_{x}{ }^{2}$ by $2 \%$, within the error of the fit.

Table 5.3 shows the results for the available data. The measurement of $\sigma_{x x^{\prime}} / \sigma_{x}{ }^{2}$ shows good agreement with what is predicted from the measured Twiss parameters for the FY2003 dataset only.

Table 5.3: Comparison of measured $\sigma_{x x^{\prime}} / \sigma_{x}^{2}$ to model.

\begin{tabular}{|c|c|c|c|c|}
\hline Run & $\beta_{P H E N I X}^{*}[\mathrm{~m}]$ & $\begin{array}{r}\sigma_{x x^{\prime}} \\
\text { design optics }\end{array}$ & $\begin{array}{l}\sigma_{x}{ }^{2}[\mu \mathrm{rad} / \mathrm{mm}] \\
\text { measured optics }\end{array}$ & data \\
\hline FY2001 & 5 & 21 & - & - \\
\hline FY2001 & 2 & 22 & $37 \pm 2$ & 一 \\
\hline FY2001 & 1 & 23 & $23 \pm 3$ & $38 \pm 2$ \\
\hline FY2002 & 3 & 21 & - & - \\
\hline FY2003 & 2 & 22 & $36 \pm 2$ & $36 \pm 2$ \\
\hline
\end{tabular}

The measured $\beta_{P H E N I X}^{*}=1 \mathrm{~m}$ optics do not agree with the measured $\sigma_{x x^{\prime}} / \sigma_{x}^{2}$ from the channeling data. Table 5.3 shows that the design value of $\sigma_{x x^{\prime}} / \sigma_{x}{ }^{2}$ only varies within $10 \%$ when $\beta_{P H E N I X}^{*}$ is changed. This is expected for the measured optics as well. The $\sigma_{x x^{\prime}} / \sigma_{x}^{2}$ of the channeling data in the $\beta_{P H E N I X}^{*}=1 \mathrm{~m}$ case agree with measured values of $\sigma_{x x^{\prime}} / \sigma_{x}^{2}$ for other $\beta_{P H E N I X}^{*}$ values. This indicates that the measured Twiss parameters for $\beta_{P H E N I X}^{*}=1 \mathrm{~m}$ have a hidden contribution which is not understood.

The average width of the channeling dip, $\sigma_{x^{\prime}}\left(x_{0}\right)$, is listed in Table 5.4. Corrections to $\sigma_{x^{\prime}}\left(x_{0}\right)$ from $x_{0}$ are less than $1 \mu \mathrm{rad}$ for RHIC parameters and crystal positions so the average is performed over all $x_{0}$.

The width of the channeling dip is between two and three times larger than expected. Simulations provide widths that are slightly larger than Equation 2.74 predicts. The size of this difference depends on the number of turns simulated. The increase is less than $10 \%$ for 40 turn simulations. However, simulations show increases 
Table 5.4: Comparison of measured $\sigma_{x^{\prime}}\left(x_{0}\right)$ to model.

\begin{tabular}{|c|c|c|c|c|c|}
\hline Run & $\beta_{P H E N I X}^{*}[\mathrm{~m}]$ & design optics & $\begin{array}{l}\sigma_{x^{\prime}}\left(x_{0}\right)[\mu \mathrm{rad} \\
\text { measured optics }\end{array}$ & simulation & data \\
\hline FY2001 & 5 & 12.3 & - & - & $39 \pm 4$ \\
\hline FY2001 & 2 & 9.98 & $19 \pm 1$ & $20 \pm 1$ & $78 \pm 4$ \\
\hline FY2001 & 1 & 8.91 & $9 \pm 1$ & $11 \pm 1$ & $35 \pm 3$ \\
\hline FY2002 & 3 & 10.8 & - & - & $58 \pm 3$ \\
\hline FY2003 & 2 & 9.98 & $14 \pm 1$ & $16 \pm 1$ & $28 \pm 2$ \\
\hline
\end{tabular}

$\sigma_{x^{\prime}}\left(x_{0}\right)$ is calculated neglecting terms of $O\left(x_{0}\right)$. For typical values of $x_{0}$ in RHIC, the error is less than $1 \mu \mathrm{rad}$ in all cases. The emittance and rms momentum spread are assumed to be the nominal RHIC values, $\epsilon=15 \pi \mathrm{mm}-\operatorname{mrad}, \sigma_{p}=0.0013$. The simulations quoted are those in Table 5.2 using the measured optics.

between $30 \%$ and $80 \%$ for simulations with 80 turns. This increase in width comes from particles that encounter the crystal multiple times. This is not included in Equation 2.74. The number of turns also affects the depth of the volume capture region, as Figure 4.7 shows, and must be matched to the data. This sets a limit on the number of turns, which in most cases is not enough to explain the whole difference.

The values of the rms momentum spread, $\sigma_{p}$, and emittance used for the calculations and simulations are assumed to be the nominal RHIC parameters listed in Table 5.4. Variations in the emittance have little effect on the width of the channeling dip for typical RHIC emittances. For RHIC parameters,

$$
\sigma_{x^{\prime}}\left(x_{0}\right) \propto 1[\mu \mathrm{rad}] \sigma_{p}
$$

This linearity continues until $\sigma_{p} \approx 7 \sigma_{p, n o m}$. However, it is not possible for the rms momentum spread to be more than a factor of 2 from the nominal value unless there is a large fraction of the beam outside of the RF bucket [73]. This was never the case during crystal studies and is not a cause of increased $\sigma_{x^{\prime}}\left(x_{0}\right)$.

Beam profile measurements using the scrapers show that the beam tails are 
broader than a gaussian distribution [53]. These measurements, discussed in Section 6.2, partially account for the increased width of the channeling peak. The nongaussian tails change the angular distribution of particles that hit the crystal. The change of the halo distribution will have a large effect on $\sigma_{x^{\prime}}\left(x_{0}\right)$. However it will have little effect on $\sigma_{x x^{\prime}} / \sigma_{x}^{2}$ because it is dominated by single particle dynamics.

A different ansatz can be used for the particle distribution in the theory, and the calculation of $\sigma_{x^{\prime}}\left(x_{0}\right)$ repeated. This was not done because the integrals involved have to be evaluated numerically in most cases. Simulations use the same distribution as the theory. More accurate simulations could be done if the details of the halo distribution were better known. No simulations were done using a different distribution.

There is a noticeable discrepancy in $\sigma_{x^{\prime}}\left(x_{0}\right)$ between the two datasets that have $\beta_{P H E N I X}^{*}=2 \mathrm{~m}$. The average width in the FY2001 run is 2.8 times larger than the average width from FY2003 run. Differences in the Twiss parameters predict only a $25 \%$ difference between the datasets. This is not understood.

The channeling efficiency is defined by dividing the depth of the channeling dip by the background rate. Table 5.5 shows the expected channeling efficiency, comparing design optics, measured optics, simulations, and the measured width of the

Table 5.5: Comparison of various models of expected channeling efficiency

\begin{tabular}{|c|c|c|c|c|c|}
\hline \multirow{2}{*}{ Run } & \multirow{2}{*}{$\beta_{\text {PHENIX }}^{*}[\mathrm{~m}]$} & \multicolumn{4}{|c|}{ Calculated Channeling Efficiency Using } \\
& 5 & $59 \%$ & - & - & $19 \pm 2 \%$ \\
\hline FY2001 & 2 & $71 \%$ & $39 \pm 2 \%$ & $37 \pm 1 \%$ & $9 \pm 1 \%$ \\
FY2001 & 1 & $74 \%$ & $75 \pm 1 \%$ & $56 \pm 3 \%$ & $20 \pm 2 \%$ \\
FY2001 & 3 & $79 \%$ & - & - & $21 \pm 1 \%$ \\
FY2002 & 2 & $71 \%$ & $52 \pm 2 \%$ & $51 \pm 1 \%$ & $26 \pm 2 \%$ \\
FY2003 & & &
\end{tabular}

The values for the efficiency for the design optics were calculated directly from Equation 2.25. The calculated angular spread is smaller than $\theta_{c}$. Equation 2.29 is not valid in this case. 
Table 5.6: Measured channeling efficiency

\begin{tabular}{|c|c|c|}
\hline Run & $\beta_{P H E N I X}^{*}[\mathrm{~m}]$ & Channeling Efficiency \\
\hline FY2001 & 5 & $24 \pm 3 \%$ \\
FY2001 & 2 & $28 \pm 3 \%$ \\
FY2001 & 1 & $19 \pm 3 \%$ \\
FY2002 & 3 & $26 \pm 3 \%$ \\
FY2003 & 2 & $26 \pm 3 \%$ \\
\hline
\end{tabular}

channeling dip. Simulations agree with the theory using the measured optics, except for $\beta_{P H E N I X}^{*}=1 \mathrm{~m}$ case. Multiple turn and dechanneling effects are particularly important in this case because of the small beam divergence on the crystal. This can lead to a lower efficiency in the simulation as compared to the theory. This also applies to discrepancies in the predicted efficiency between the theory in Table 5.5 and the simulations in 5.2.

Table 5.6 shows the average measured channeling efficiency from the data for each $\beta_{P H E N I X}^{*}$. The efficiency does not match any theory or simulations. This disagreement stems from the disagreement in the value of $\sigma_{x^{\prime}}\left(x_{0}\right)$. If the measured value of $\sigma_{x^{\prime}}\left(x_{0}\right)$ is used to calculate the channeling efficiency, the agreement is very good for four of the runs. The measured efficiency of the $\beta_{P H E N I X}^{*}=2 \mathrm{~m}$ data in the FY2001 run is 3 times larger than the expected efficiency using the measured width. It is, however, consistent with the measured efficiency in the FY2003 data with $\beta_{P H E N I X}^{*}=2 \mathrm{~m}$. This indicates a contribution to the $\sigma_{x^{\prime}}\left(x_{0}\right)$ that is not understood in the FY2001 $\beta_{P H E N I X}^{*}=2 \mathrm{~m}$ dataset. Nevertheless, the agreement between the channeling efficiency and width of the channeling dip shows that the physics of crystal channeling are understood. This analysis shows that proper understanding of $\sigma_{x^{\prime}}\left(x_{0}\right)$ is essential to predicting the channeling efficiency.

Simulations show that the bend angle of the crystal can be determined by

$$
\theta_{b}=\theta_{2}-\theta_{1}
$$


where $\theta_{1,2}$ are the centers of the two lorentzians in the fit. Simulations use a bend angle of $\theta_{b}=388 \mu \mathrm{rad}$ to match the volume capture region. The bend angle was also measured by reflecting a laser beam off the crystal face and measuring the deflection as the crystal is moved perpendicular to the laser. The results of this are given in Table 5.7. Crystal 2 was used during the FY2003 run. Crystal 1 was used during the other runs. The laser measurement of Crystal 1 is poor because the reflected beam spot was diffuse, even after cleaning the crystal surface. The quality of measurement of Crystal 2 is much better.

Table 5.7: Measurements of the crystal bend angle

\begin{tabular}{|c|c|c|}
\hline Crystal Number & $\begin{array}{c}\text { Channeling } \\
\text { Measurement }[\mu \mathrm{rad}]\end{array}$ & $\begin{array}{c}\text { Reflected Laser } \\
\text { Measurement }[\mu \mathrm{rad}]\end{array}$ \\
\hline 1 & $439.8 \pm 0.4$ & $591 \pm 220$ \\
2 & $436.7 \pm 0.8$ & $433 \pm 96$ \\
\hline
\end{tabular}

The difference between the simulations and the channeling data can be traced to difficulties in determining $\theta_{2}$. The height of the second lorentzian is determined by the height of the volume capture region. The scatter in the points near the end of the volume capture region is such that the position, $\theta_{2}$, and the width, $w_{2}$, are best fit when $w_{2}$ is small. This will increase the measured bend angle. The measurements indicate that laser measurements of the bend angles of the crystals are consistent within the error bars with both the simulations and data. It was hoped that by measuring the angle with a laser, a check of the channeling data could be done. However, the large error bars from the laser measurement prevent any meaningful comparison.

Crystal channeling at RHIC shows some agreement with simple models and simulations. Using the correct Twiss parameters, it is possible to predict the location of the channeling dip as a function of the distance between the beam center and 
crystal. Predictions of $\sigma_{x^{\prime}}\left(x_{0}\right)$ are dependent on the Twiss parameters and ansatz beam distribution. Predictions of the channeling efficiency can be made knowing $\sigma_{x^{\prime}}\left(x_{0}\right)$. The predicted efficiency may be larger than the measured efficiency because of the effect of multiple encounters with the crystal and inadequate knowledge of the beam halo distribution. Measurements of the width mostly agree with the measured efficiency according to Equation 2.28.

\subsection{Crystal Collimation and Background Reduction}

The copper scraper must be positioned to intercept the channeled particles, once the crystal collimator is properly aligned to the beam halo. If the channeling efficiency is low, as in the case of RHIC, the scraper must also intercept the particles that scatter from the crystal. Figure 5.7 shows a simulation of the distribution of particles in the $\mathrm{x}-\mathrm{y}$ plane at the location of the scraper after passing through the crystal for $\beta_{P H E N I X}^{*}=2 \mathrm{~m}$. The crystal is located at $-17.6 \mathrm{~mm}$ from the beam center, $7 \mathrm{~m}$ upstream of the scraper location in this simulation. The scraper enters

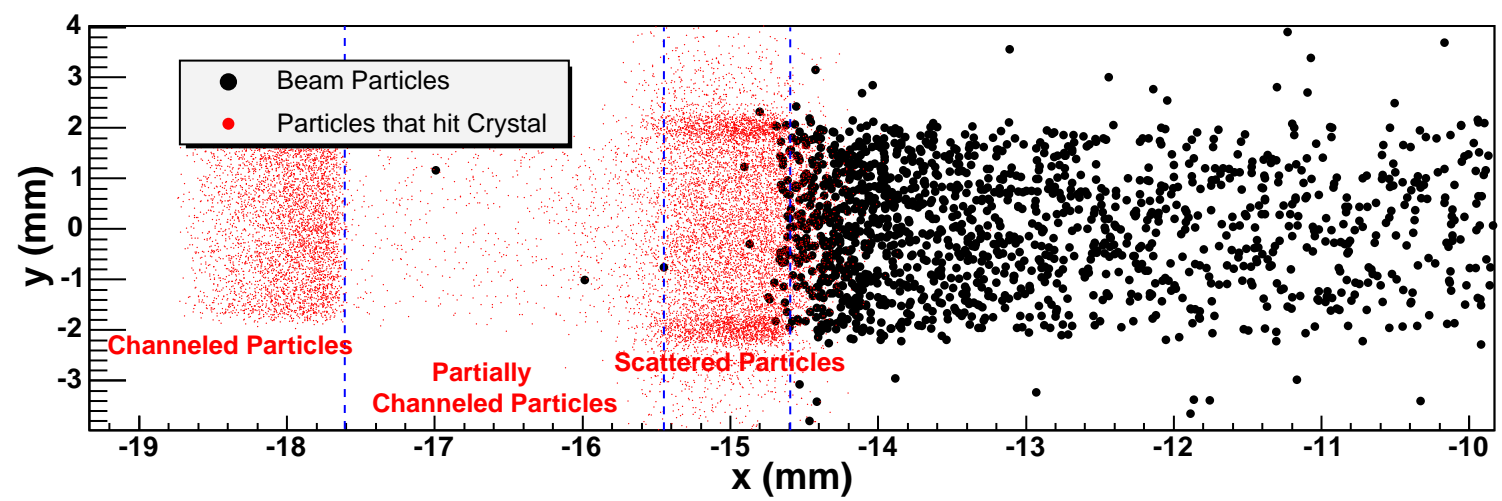

Figure 5.7: Simulated particle distribution at the scraper location after passing through the crystal. 
the figure from the left. The simulation shows the large separation of the channeled particles from the scattered particles. Because of the low channeling efficiency, the scraper must be moved very close to the beam to intercept all of the particles. If the channeling efficiency were larger, more particles would be located to the left of the figure. However, since some particles strike the crystal outside of the angular acceptance, there will always be particles to the right.

Figure 5.8 shows the upstream and downstream PIN diodes as a function of time during Fill 03061 during the FY2003 run as the scraper is moved relative to the crystal collimator. At the left of the figure, the crystal and scraper are inserted into the beam. The crystal is not aligned to the beam and is not channeling. At the first dotted red line the crystal is rotated into the channeling position, which was determined by a prior crystal scan. The scraper is not inserted far enough to intercept the channeled particles, which pass the scraper, so that the downstream PIN diode rate goes down.

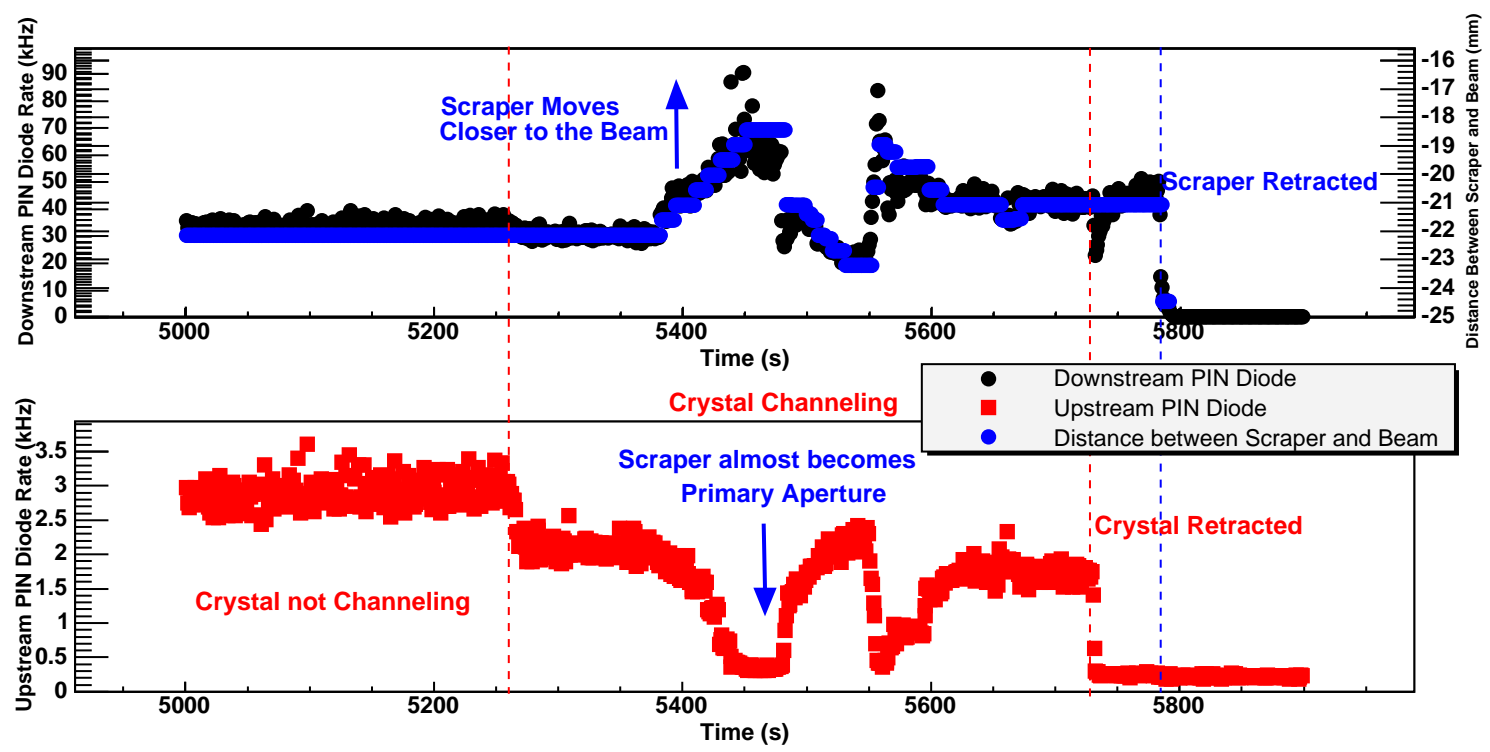

Figure 5.8: PIN diode rates during Fill 03061 when the scraper is moved relative to the crystal. The crystal is $13.6 \mathrm{~mm}$ from the center of the beam. 
As the scraper is moved closer to the beam, the number of particles hitting the scraper increases. The downstream PIN diode rate increases and the upstream PIN diode rate decreases as less particles hit the crystal. The scraper is moved in until it almost becomes the primary aperture, indicated by the upstream PIN diodes approaching the level were the crystal is fully retracted. After a partial retraction, insertion, and another partial retraction of the scraper, at the second dotted red line the crystal is retracted fully. The upstream PIN diodes decrease to background levels. Then the scraper is removed, and the downstream PIN diodes drop to background levels. It is possible to use both sets of PIN diodes to understand the placement of the scraper with respect to the crystal.

Various background signals were recorded from each experiment to measure the effectiveness of the crystal collimator in removing the background. The STAR detector is located directly downstream of the crystal collimator and is therefore most sensitive to its effects. During the FY2001 and FY2002 runs, the STAR Central Trigger Barrel (CTB) was used for measuring background level in the detector. For the FY2003 run, the STAR experiment provided gated Beam-Beam Counter (BBC) signals called "yellow halo" and "blue halo". These gated signals detect particles traveling through the detector in in the direction of the yellow and blue beams respectively. The yellow halo signal was used during FY2003 run for these measurements.

These measurements were conducted during four stores using gold and proton beams. The crystal collimator and copper scraper were positioned in the beam halo. The crystal angle would be scanned to align the crystal to the beam. The scraper would then be moved relative to the crystal. For three of the stores $(01825,01835$, and 02185), the crystal was scanned again to measure the effect of crystal angle on the STAR background. For the remaining store (03061), the crystal remained in the aligned position. The crystal and scraper position was inserted further. Several times, 


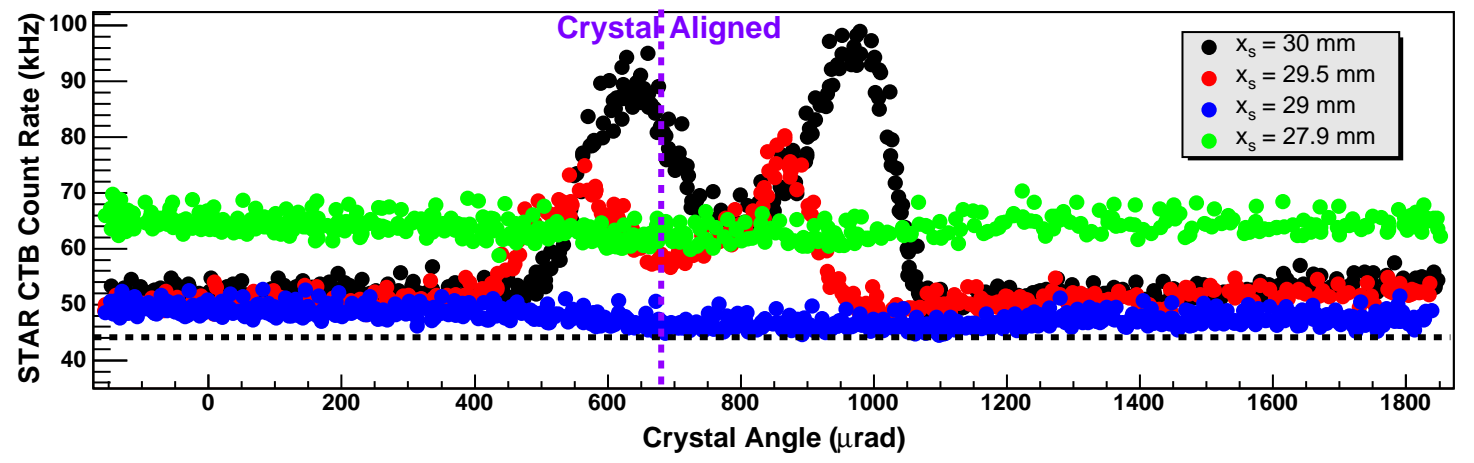

Figure 5.9: STAR CTB signal during four crystal scans during Fill 02185. The crystal angle is aligned at $680 \mu \mathrm{rad} . x_{s}$ is the scraper position. The black dotted line represents the CTB background without crystal and scraper. The purple dotted line shows the angle where the crystal is aligned to the beam.

the crystal and scraper were retracted to measure the STAR background without any collimation.

Figure 5.9 shows the response of the STAR CTB during four crystal scans in Fill 02185. The crystal angle is aligned at $680 \mu \mathrm{rad}$. When the scraper is far from the beam, it is possible for particles channeled by the crystal to travel to the STAR detector and cause background. As the scraper is moved closer to the beam, the number of particles hitting the detector is reduced and the background peaks are asymmetrically truncated as the crystal in scanned. This asymmetry is caused by the changing angle of the channeled beam during the scan. The channeled particles will hit different apertures as they travel through the machine to STAR.

When the scraper is $29 \mathrm{~mm}$ from the beam, the STAR background is slightly reduced when the crystal is channeling. However, as the scraper is inserted further into the beam to $27.9 \mathrm{~mm}$, it is inserted too far into the beam and actually causes excess background.

The other RHIC experiments are negligibly affected. Figure 5.10 shows selected backgrounds from each of the experiments during the same time as the black trace 

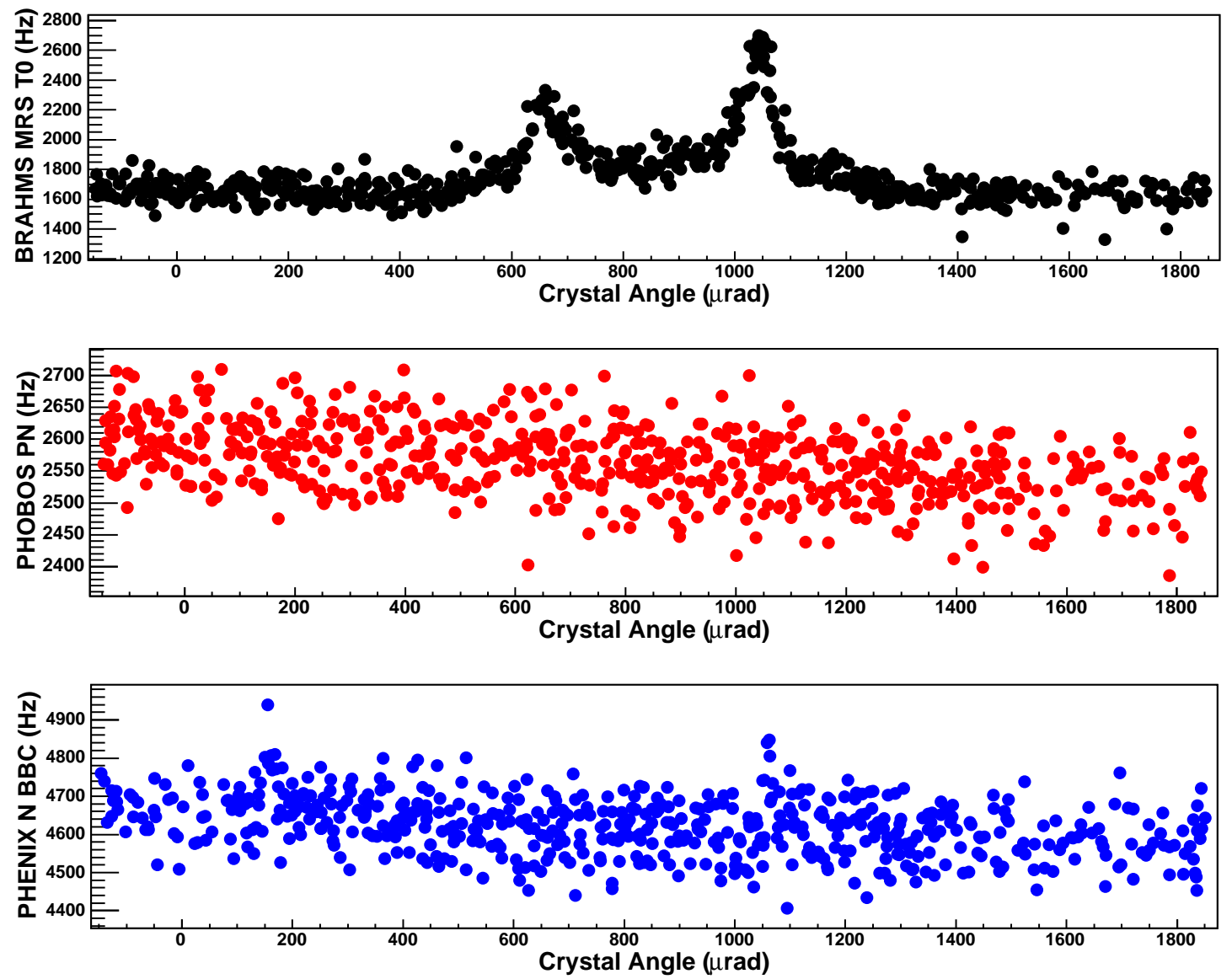

Figure 5.10: Selected background signals from the remaining RHIC experiments during the black trace in Figure 5.9.

in Figure 5.9. The BRAHMS Mid-Rapidity Spectrometer T0 (MRST0), PHOBOS South Paddle Counter (PN), and PHENIX North Beam-Beam Counter (NBBC) are chosen to highlight the typical response of the background during crystal collimation. Only the BRAHMS experiment sees any effect of the crystal scan. The effect on PHOBOS and PHENIX is negligible.

Table 5.8 shows the phase advance $\left(\bmod 360^{\circ}\right)$ between the crystal and the various experiments. From this table, it is not surprising that BRAHMS and STAR see similar effects. Any scattered particles produced at the crystal are at the same horizontal betatron phase at these experiments. STAR, being closer, sees the larger 
Table 5.8: Relative phase advance between Crystal Collimator and RHIC experiments from Design Optics

\begin{tabular}{|c|c|}
\hline Experiment & $\Delta \phi \bmod 360^{\circ}$ \\
\hline STAR & $320^{\circ}$ \\
PHENIX & $80^{\circ}$ \\
PHOBOS & $175^{\circ}$ \\
BRAHMS & $320^{\circ}$ \\
\hline
\end{tabular}

effect. At PHOBOS scattered particles are at approximately the opposite phase as at STAR and BRAHMS and should see some effect. However, PHOBOS is almost one complete revolution away from the crystal and most of the scattered particles are lost. The PHOBOS detector is located very close to the beam pipe and did not seem to be sensitive to some of the backgrounds that affected the other experiments, such as scattering in the triplet magnets. The PHENIX experiment is immediately upstream of the crystal collimator and is least sensitive to particle scatter from the collimator.

The crystal collimation system was rarely successful in reducing the background in the STAR detector, even when everything was optimally positioned. Figure 5.11 shows the background of STAR as measured with its yellow halo signal during Fill 03061 at the same time as Figure 5.8. The dotted black line shows the background when the crystal and scraper are retracted. At the left of the figure, the crystal and scraper are in the beam halo. The crystal is not channeling. The crystal is then aligned to channel the beam and increases the STAR background by a factor of two. The scraper is inserted until it becomes the primary aperture, then partially retracted, inserted, and partially retracted again. The crystal is retracted fully, followed by the scraper. Figure 5.11 shows that the scraper alone is more effective in reducing the background than the crystal and scraper together. This is the case regardless whether the crystal is channeling or scattering. 


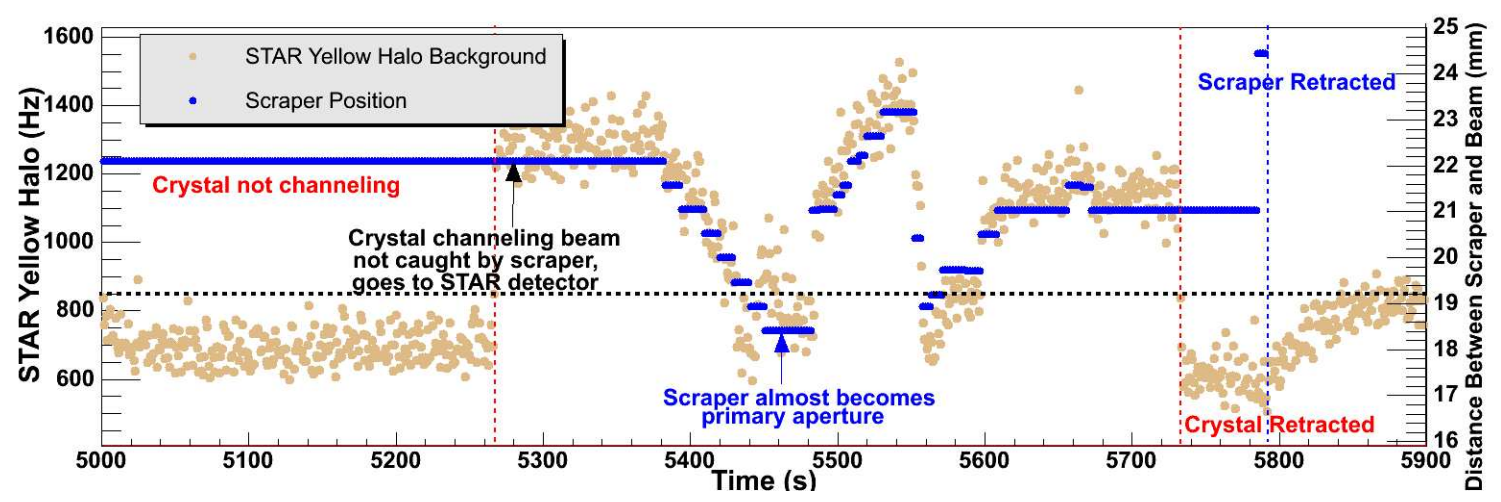

Figure 5.11: STAR yellow halo signal during the crystal collimation attempt in Figure 5.8. The scraper is closer to the beam toward the bottom of the graph.

Figure 5.12 shows the ratio of the STAR background with the crystal channeling to the uncollimated background vs. the difference of the normalized positions of the crystal and the scraper. A negative relative position indicates that the crystal is the primary aperture. At zero, the crystal and scraper have the same betatron amplitude. The vertical error bars are statistical. As the scraper comes closer to the beam, the STAR background decreases, but rarely drops below the level of the uncollimated background indicated by the red dotted line. The few times that crystal collimation is successful, shown by points below the dotted red line, are not understood. These

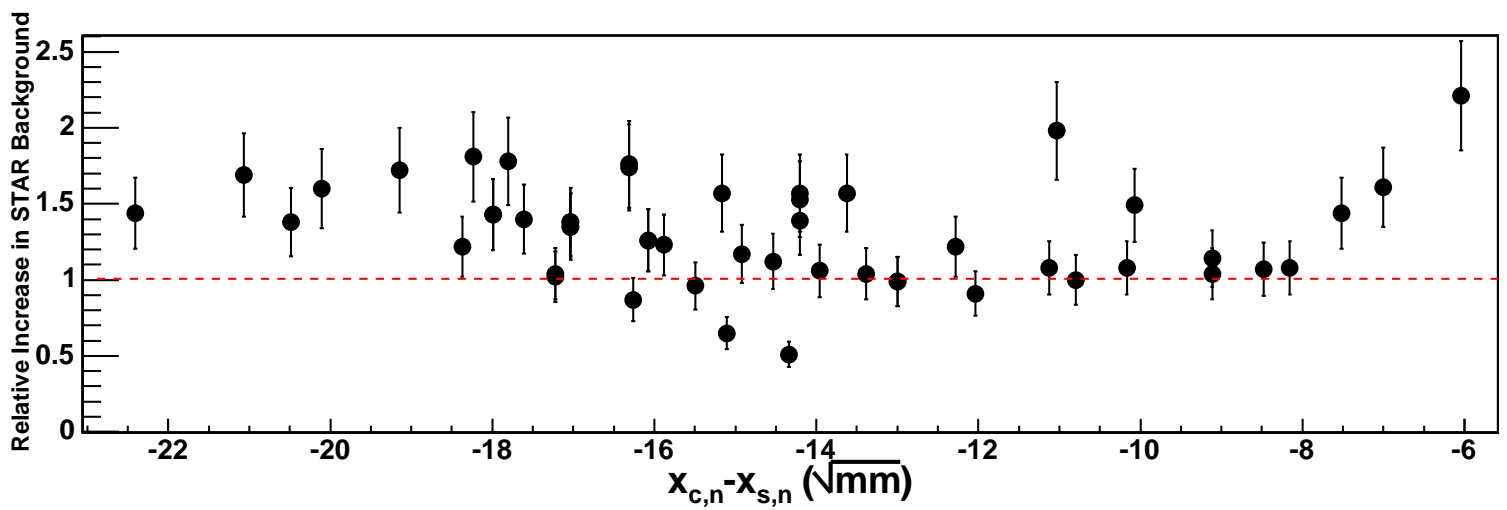

Figure 5.12: The relative increase of the STAR background vs. the relative normalized position between the crystal and the scraper. The red line indicates where the crystal collimated background equals the collimated background. The crystal collimator almost never reduced the background, in most cases the background rose. 
points are taken within a five minute interval, along with other points. There is no a priori reason that these points should be more successful than the others. Nothing special occurred during these data points to make collimation more effective.

Most of the time crystal collimation was not better than no collimation at all. The reason that the crystal does not act as a good collimator is the low channeling efficiency. The large amount of scattering caused by the crystal cannot be removed with the scraper. If the channeling efficiency were increased, it is possible that the crystal would have an increased benefit. The possibility of moving the crystal to another location in RHIC was discussed. All warm sections in RHIC contain similar Twiss parameters. Placing the crystal in a cold region with more favorable optics would require expensive cryogenic bypasses. Adding more secondary collimators to intercept the scattered particles from the crystal collimator may alleviate the problem. In this case, the crystal collimator acts no differently than an ordinary scraper. We decided that the crystal collimator would be removed and the collimation system upgraded. This is the topic of Chapter 7 . 


\section{Chapter 6}

\section{Collimation}

This chapter details several measurements that were made using the copper scrapers through the FY2003 RHIC run. Initial efforts that focused on system commissioning are discussed in Section 3.6.

\subsection{PIN Diode Calibration}

The DC Current Transformer (DCCT) [74], which measures the total beam current, is insensitive to beam losses of less than $10^{6} \mathrm{Au}$ ions. The collimator PIN diodes can be used to measure small losses immediately downstream of the collimators. It was hoped that the PIN diode rate could be calibrated to the beam loss rate so that small losses from collimation could be more easily quantified. The attempt to calibrate the PIN diode signal with the DCCT is fully documented in Reference [53].

One may expect to calibrate the PIN diodes by equating the instantaneous rate of the diodes with the slope of the beam current. This approach has two flaws. The first is that the DCCT will measure a loss anywhere in RHIC whereas the PIN 


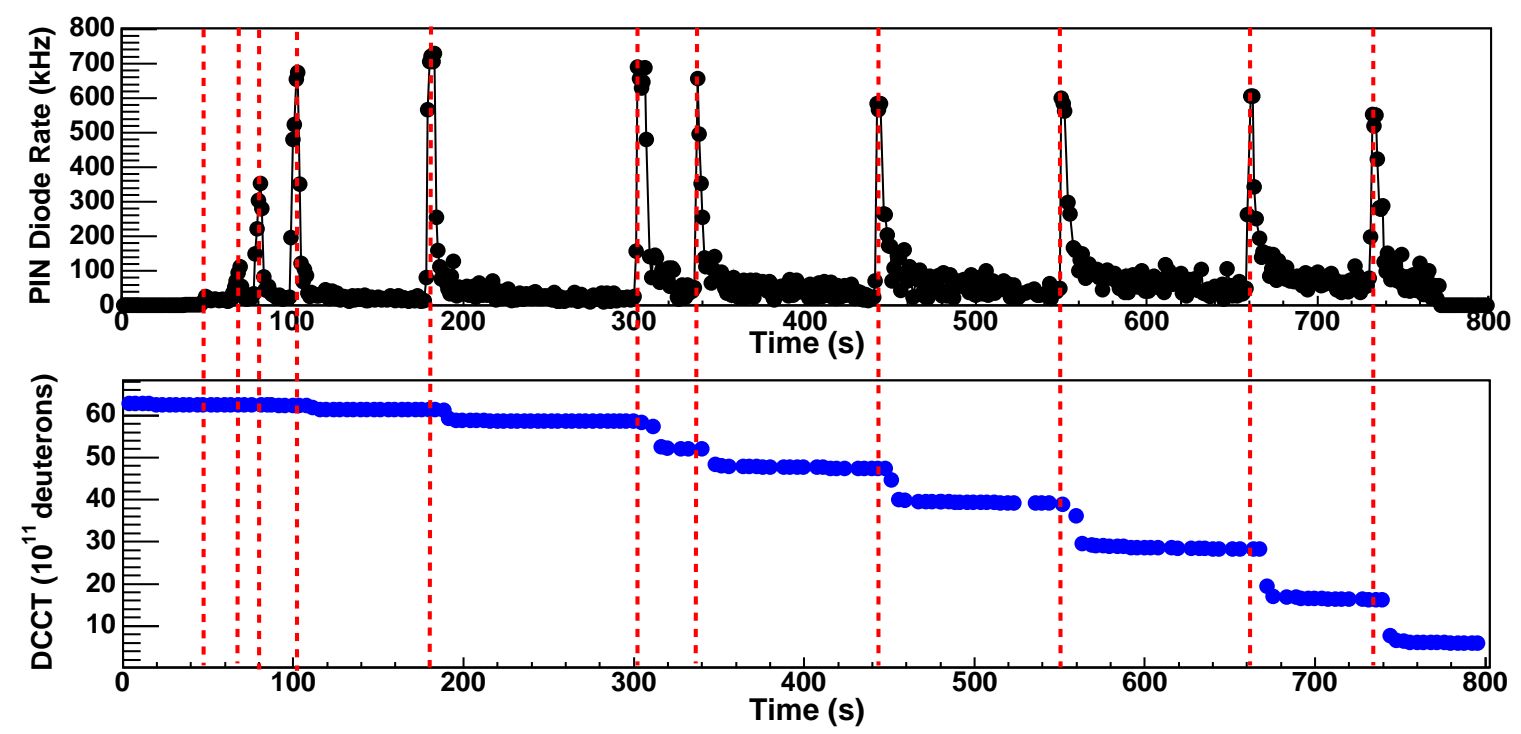

Figure 6.1: PIN Diode rate and Beam Current for Store 03254 in the Blue ring. The dashed lines show the times when the scraper was moved. The spikes in the PIN diode rate occur when the scraper moved. The DC Current Transformer lags a few seconds after the scraper moves.

diodes only measure losses near the collimators. By using the collimators to remove the beam, this effect can be avoided because most of the losses will occur at the collimators. The second flaw is seen in Figure 6.1. The drop in DCCT current happens a few seconds after the PIN diode spike. The instantaneous PIN diode rate is not directly correlated to the DCCT slope.

Another approach to calibrate the PIN diodes is to measure the height of the PIN diode spike and to correlate it with subsequent beam loss. This way, each spike can be correlated with each drop in current. Two attempts at this are shown in Figure 6.2. Of the eight data sets used for this analysis, only the data set taken on September 3, 2000 shows a correlation. These data were taken with one bunch with an intensity $0.45 \times 10^{9}$ ions. Because it is the only data set taken with one bunch, the losses were much smaller, so that the PIN diode rates stayed below $150 \mathrm{kHz}$. All other datasets had loss rates that were larger than this. 

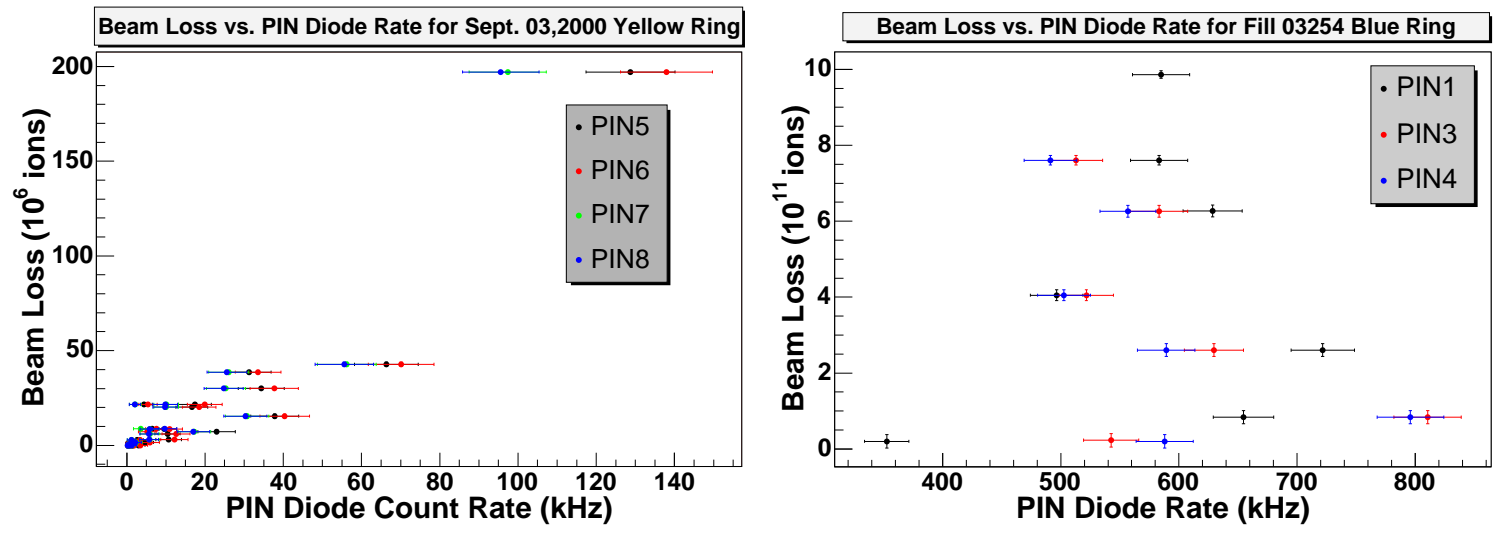

Figure 6.2: Beam losses vs. PIN diode rate for two fills. The left graph is data taken on September 3, 2000. The right is for Fill 03254

Figure 6.2 shows that the PIN diodes may be calibrated if they have a rate of less than $100 \mathrm{kHz}$. The PIN diodes have a maximum count rate of $1 \mathrm{MHz}$. When count rates approach this rate, pulse pileup becomes an issue and causes the PIN diodes to miscount. When the loss rates increase, the loss pattern continues into the arc and the PIN diodes see less of the total loss. This is discussed in Section 6.3. Both effects cause variation in the PIN diode rate and make calibration in this regime difficult.

A linear fit to the average PIN diode rate for the September 3, 2000 fill is shown

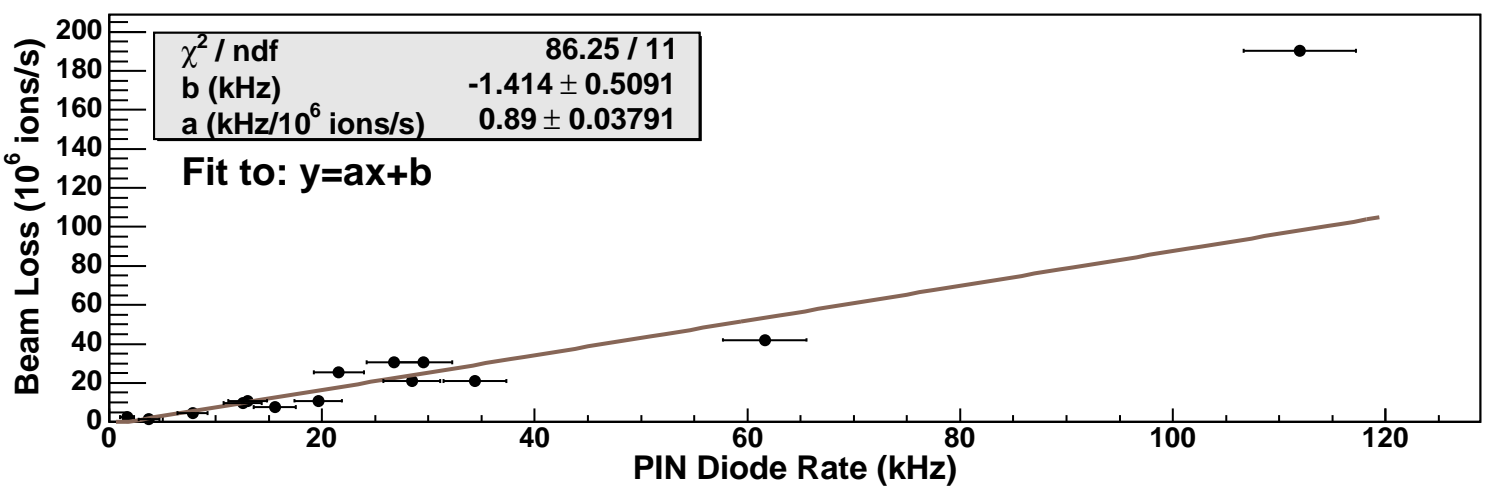

Figure 6.3: Linear fit to average PIN diode rate from the Fill on September 3, 2000. The fit does not use the point at $112 \mathrm{kHz}$. 
in Figure 6.3. It is possible to estimate the amount of beam being lost for typical PIN diode rates during collimation or experiments using the scraper in the beam halo. Our confidence in the calibration is limited because we were not able to repeat the measurement. It was never used as a result.

The upgrade of the collimation system requires a new calibration because the relationship between the PIN diodes and the individual scrapers is different. This new calibration, however, will only be useful for the PIN diodes that look at the primary collimator. PIN diodes for the secondary collimators see losses caused by previous collimators as well, and a direct correlation with beam current will not be possible if other collimators are inserted.

\subsection{Beam Profile Measurements}

It is possible to measure the transverse profile of the beam by using the collimator to scrape away the beam. Using this technique it is possible to check the calibration of the Ionization Profile Monitor (IPM). The theory of measuring a beam profile using a collimator is discussed in References [33, 53]. This section follows Reference [53].

Assume the one dimensional transverse phase space distribution $f\left(x, x^{\prime}\right)$ is normalized to the total number of particles in the beam, $N_{0}$. The transverse beam profile in one dimension is then

$$
\rho(x)=\int_{-\infty}^{+\infty} \mathrm{d} x^{\prime} f\left(x, x^{\prime}\right) .
$$

By changing variables to

$$
u^{2}=\frac{x^{2}}{\beta}+\left(\frac{\alpha}{\sqrt{\beta}} x+\sqrt{\beta} x^{\prime}\right)^{2}
$$


$f\left(x, x^{\prime}\right)$ becomes a function of $u$ alone, denoted as $\tilde{f}(u)$. Physically, $u$ is twice the particle action. Dispersion is ignored. Equation 6.1 becomes

$$
\rho(x)=\int_{\frac{x}{\sqrt{\beta}}}^{\infty} \mathrm{d} u \frac{u \tilde{f}(u)}{\sqrt{\beta u^{2}-x^{2}}} .
$$

If a collimator is inserted to a position $x_{c}=u_{c} \sqrt{\beta}$ the number of surviving particles is

$$
N\left(u_{c}\right)=2 \pi \int_{0}^{u_{c}} u \tilde{f}(u) \mathrm{d} u .
$$

The factor of $2 \pi$ comes from the angular integral around the normalized phase ellipse. The number of particles lost by moving the collimator is

$$
\frac{\mathrm{d} N\left(u_{c}\right)}{\mathrm{d} u_{c}}=2 \pi u_{c} \tilde{f}\left(u_{c}\right)
$$

Combining the above equation with Equation 6.3 gives the beam profile in terms of the particle loss

$$
\rho(x)=\frac{1}{\pi} \int_{u_{c}}^{\infty} \frac{\mathrm{d} N\left(u_{c}\right)}{\mathrm{d} u_{c}} \frac{\mathrm{d} u}{\sqrt{\beta u^{2}-x^{2}}} .
$$

It is possible to reconstruct the beam profile using Equation 6.6 by measuring the beam loss rate as a function of collimator position.

For a gaussian beam in phase space, $\tilde{f}(u)$ is given by

$$
\tilde{f}(u)=\frac{N_{0}}{2 \pi \sigma_{u}^{2}} \exp \left[-\frac{u^{2}}{2 \sigma_{u}^{2}}\right]
$$

and the number of surviving particles as a function of collimator position becomes

$$
N\left(x_{c}\right)=N_{0}\left(1-\exp \left[-\frac{x_{c}^{2}}{2 \sigma_{x}^{2}}\right]\right)
$$


where $\sigma_{x}=\sigma_{u} \sqrt{\beta}$ is the rms beam size. Equation 6.6 can be solved analytically to give

$$
\begin{aligned}
\rho(x) & =\frac{N_{0}}{\sqrt{2 \pi} \sigma_{x}} \exp \left[-\frac{u^{2}}{2 \sigma_{x}^{2}}\right] \\
& =\frac{N_{0}-N(x)}{\sqrt{2 \pi} \sigma_{x}} .
\end{aligned}
$$

In this case, by making a plot of the beam current as a function of the collimator position, one can obtain the rms beam size by fitting Equation 6.8 to the data. For beams with a more complex phase space distribution Equation 6.6 must be solved numerically.

Table 6.1 lists the existing data used to measure the beam profiles in RHIC. Each of the data sets were taken using the horizontal collimator. The vertical collimator was not used because it did not have enough travel to remove all of the beam.

Table 6.1: Beam Profile Data Sets

\begin{tabular}{|c|c|c|c|c|c|}
\hline Store & Ring & Species & Energy & $\sigma(\mathrm{mm})$ & $\epsilon(\pi \mathrm{mm}-\mathrm{mrad})$ \\
\hline Sept. 3, 2000 & Yellow & $\mathrm{Au}$ & Injection & $4.013 \pm 0.003$ & $11 \pm 1$ \\
02233 & Blue & $\mathrm{p}$ & Store & $5.266 \pm 0.005$ & $67 \pm 7$ \\
02320 & Yellow & $\mathrm{p}$ & Store & $3.183 \pm 0.004$ & $24 \pm 2$ \\
02320 & Blue & $\mathrm{p}$ & Store & $4.129 \pm 0.003$ & $41 \pm 4$ \\
03254 & Yellow & $\mathrm{Au}$ & Injection & $4.526 \pm 0.013$ & $16 \pm 2$ \\
03254 & Blue & $\mathrm{d}$ & Injection & $5.370 \pm 0.004$ & $22 \pm 2$ \\
03750 & Yellow & $\mathrm{p}$ & Store & $2.721 \pm 0.079$ & $18 \pm 2$ \\
03750 & Blue & $\mathrm{p}$ & Store & $1.509 \pm 0.006$ & $5.5 \pm 0.5$ \\
\hline
\end{tabular}

Figure 6.4 shows the data from Fill 03254 in the yellow ring. The error bars are the same size as the data points. The line is a fit to Equation 6.8, and matches the data well. Figure 6.5 shows the data from Fill 02233 in the Blue ring. The fit is reasonable, but does not fit the start of the loss (the beam tails) very well. One should also notice that only $44 \%$ of the beam is removed in Figure 6.5 whereas $80 \%$ is scraped away in Figure 6.4. 


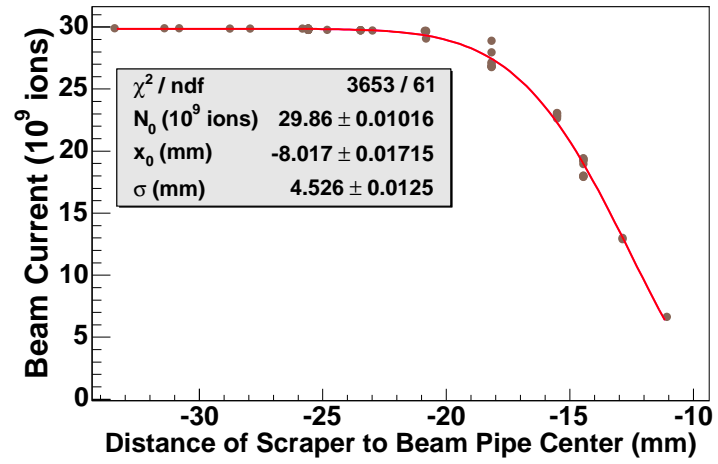

Figure 6.4: Data from the yellow ring with gold for Fill 03254

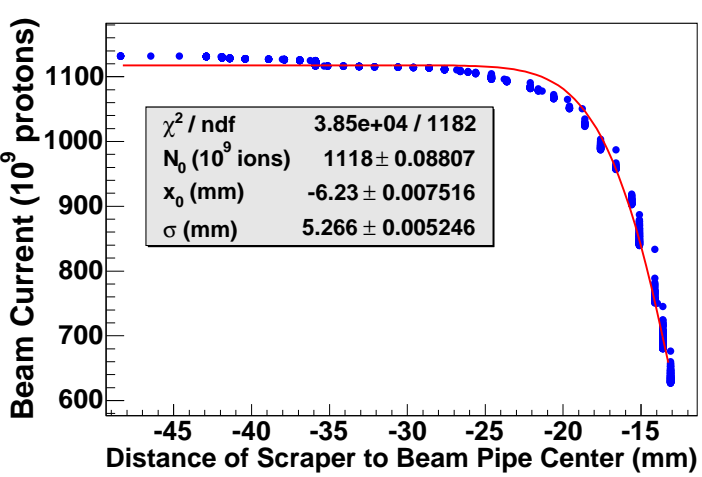

Figure 6.5: Data from the Blue ring with protons for Fill 02233

The error bars on the beam size are from the fit, and the emittance error is dominated by the uncertainty in the $\beta$ function at the collimator which is approximately $10 \%$ [75]. All of the data with proton beams were taken in preparation for or during PP2PP runs to reduce the emittance of the beam. For these fills, the beams were not fully scraped away and the fits were entirely in the tails of the beam. These fits are dominated by the non-gaussian tail and give a large value of the beam size. The gold and deuteron beams were almost fully scraped away. When the beams were fully scraped away, the non-gaussian tails matter less and the emittances agree with typical values for RHIC for the gold and deuteron beams [76]. This shows that for this technique to be successful, as much of the beam as possible needs to be removed with the scraper.

Table 6.2 compares emittance measurements using the IPM to the collimator measurements. Many of the stores did not have data logged for the IPM measure-

Table 6.2: Comparison of Collimator and IPM horizontal emittance measurement

\begin{tabular}{|c|c|c|c|}
\hline Store & Ring & Collimator Measurement & IPM Measurement \\
\hline 02233 & blue & $67 \pm 7 \pi \mathrm{mm}-\mathrm{mrad}$ & $55 \pm 7 \pi \mathrm{mm}-\mathrm{mrad}$ \\
02320 & blue & $24 \pm 2 \pi \mathrm{mm}-\mathrm{mrad}$ & $33 \pm 5 \pi \mathrm{mm}-\mathrm{mrad}$ \\
02320 & yellow & $41 \pm 4 \pi \mathrm{mm}-\mathrm{mrad}$ & $55 \pm 7 \pi \mathrm{mm}-\mathrm{mrad}$ \\
\hline
\end{tabular}


ments. The error in the IPM measurement is due to the error in the $\beta$ function at the IPM and errors in measuring the beam size. The IPM measurements agree with the collimator measurement to approximately $30 \%$. Considering that the beam was not fully removed in these cases, the collimator measurement agrees with the IPM reasonably well. Unfortunately, there is no logged IPM data for fills where the beam was fully scraped away to compare with.

\subsection{Collimator Efficiency}

The scrapers were used in a variety of ways to alleviate beam background issues during each of the RHIC runs. The scrapers were used during the:

- FY2001 gold run to reduce beam scraping at the abort kickers on the ramp during $\beta$ squeeze [77].

- FY2002 polarized proton run for emittance reduction for the PP2PP run.

- FY2003 deuteron-gold run for experimental background reduction [78].

- FY2003 polarized proton run for experimental background reduction and emittance reduction for the PP2PP run.

The lower beam intensity during the FY2001 and FY2002 runs caused low background in the detectors, and collimation during a store was unnecessary. However, orbit deviations during the energy ramp caused beam scraping at the abort kickers in each ring. The top plot of Figure 6.6 shows scraping at the abort kickers on the ramp around $s \approx 1230 \mathrm{~m}$ for Yellow and $s \approx 1320 \mathrm{~m}$ for Blue. The vertical scale is truncated at $30 \mathrm{Rad} /$ hour to show smaller losses at other locations in the ring. Losses can typically reach approximately $2000 \mathrm{Rad} /$ hour on the ramp. The scrapers are used in the middle graph of Figure 6.6. Neglecting losses around transition, 


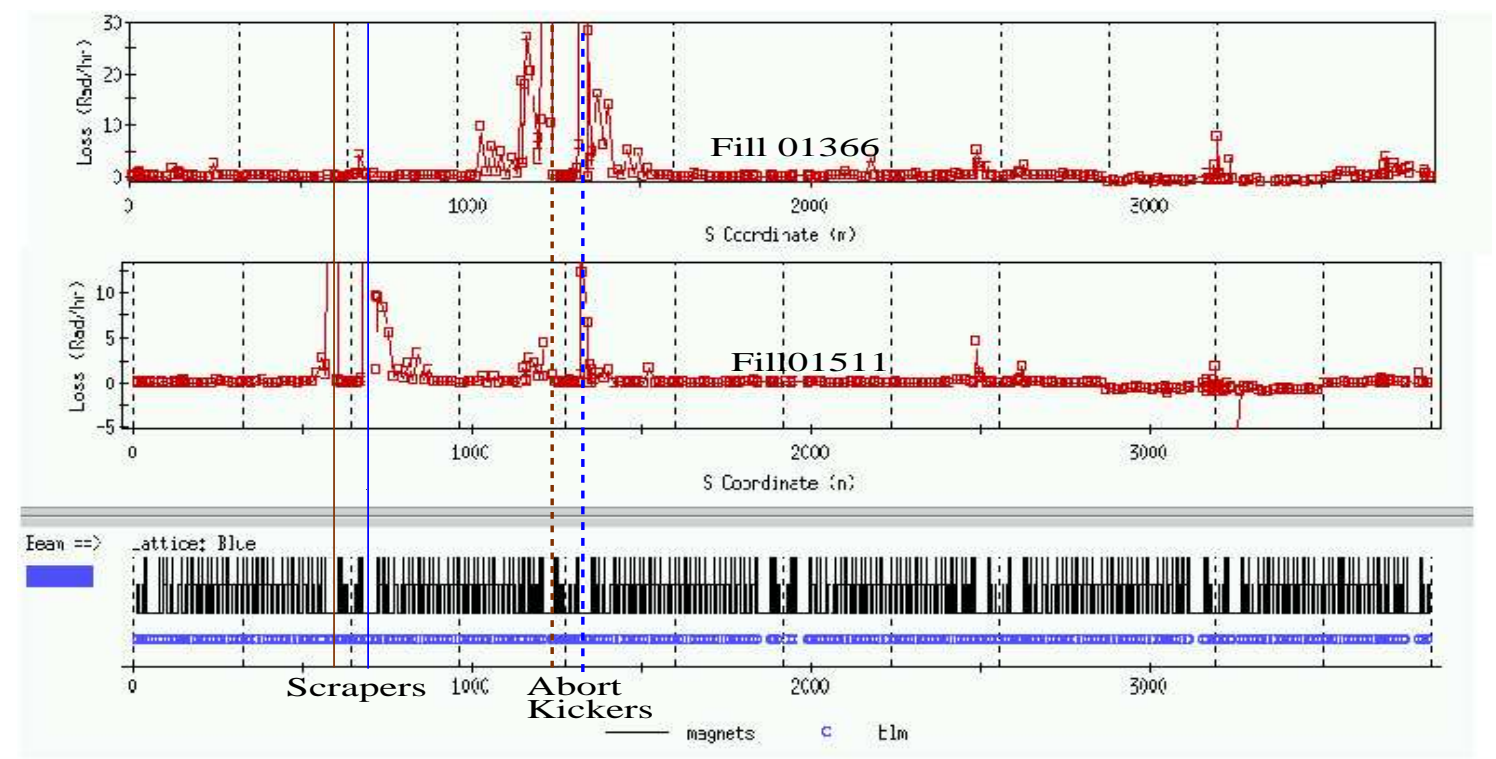

Figure 6.6: Losses around the RHIC ring on two ramps during FY2001. The top plot shows beam losses on a ramp with beam scraping at the abort kickers (blue and yellow dashed lines). The middle graph shows beam losses on a ramp with the scrapers inserted (blue and yellow solid lines). The bottom graph shows the lattice. Black dotted lines denote the interaction regions and centers of the arcs. The vertical scales are truncated.

losses at the kickers are reduced to approximately $20 \mathrm{Rad} /$ hour during the ramp. The scrapers usually see loss rates of approximately 1000 Rad/hour. Starting with Fill 01492, the scrapers were routinely set to a predefined position before the start of any ramp.

The averaged singles rate from the Zero Degree Calorimeters (ZDC) [79] for each experiment were used to measure experimental backgrounds during the FY2001 run. Figure 6.7 shows this background normalized by the collision rate at each RHIC experiment during Fill 01759 while the yellow horizontal scraper is inserted into the beam. Only the PHENIX background rate is affected as the scraper is inserted into the beam. The other experiments were largely unaffected by collimation. This was typical behavior for the FY2001 and FY2002 runs.

The ZDCs are located downstream of the DX magnet, between the blue and yellow rings on either side of each detector. The ZDC singles rate does not neces- 

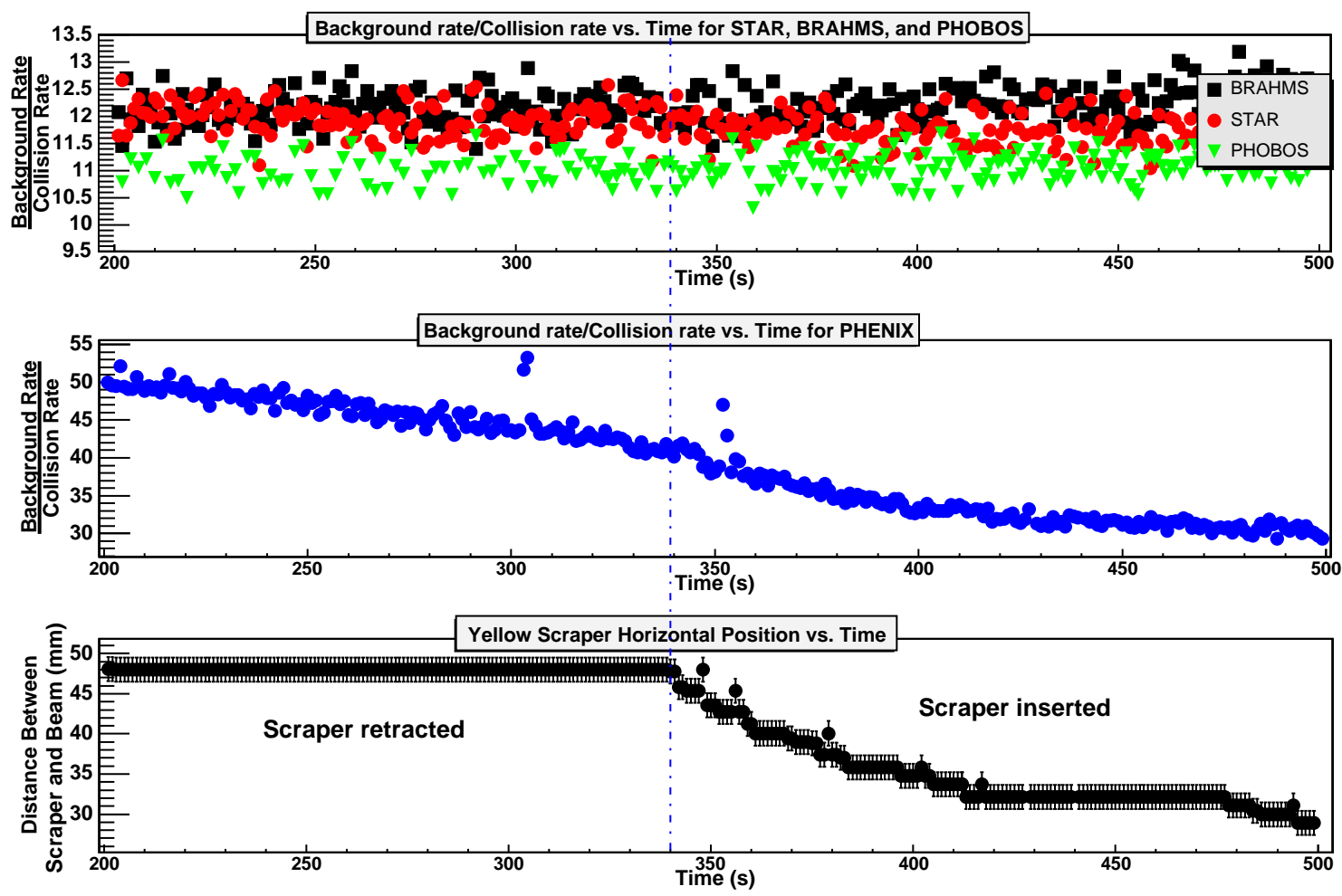

Figure 6.7: Experimental backgrounds during Fill 01759 in the FY2001 Au run. The top figure shows BRAHMS, STAR and PHOBOS backgrounds, the middle shows PHENIX. The bottom graph shows the scraper position. Only PHENIX benefits from collimation.

sarily indicate the backgrounds that concern the experiments because of its forward and shielded location. In particular, the Muon Identification (MuID) arms of the PHENIX detector are sensitive to particle flow not measured by the ZDCs. The MuID detectors are $12.5 \mathrm{~m}$ long by $9.8 \mathrm{~m}$ wide wall consisting of 5 layers of steel with detectors sandwiched between the layers and are approximately $7 \mathrm{~m}$ from the interaction point on either side of the detector [80]. A study was done at the end of the FY2002 run with the PHENIX detector to measure the effects of the scrapers on the background in the PHENIX MuID [77]. That study showed that the scrapers initially reduced the MuID trigger rate by a factor of about eight.

The RHIC experiments provided more relevant background signals for subsequent runs. These signals were generally derived from detectors that were more 
Table 6.3: Experimental detectors used for background measurement

\begin{tabular}{|c|c|c|c|c|c|}
\hline Detector & Name & Type & $\begin{array}{c}\text { Longitudinal } \\
\text { Distance to IP }\end{array}$ & $\begin{array}{c}\text { Transverse Distance } \\
\text { from Centerline }\end{array}$ & $\begin{array}{c}\text { Detector } \\
\text { Area }\end{array}$ \\
\hline ZDC & & $\begin{array}{c}\text { tungsten/fiber } \\
\text { calorimeter }\end{array}$ & $\pm 40 \mathrm{~m}$ & - & $100 \mathrm{~cm}^{2}$ \\
STAR & BBC & Scintillators & $\pm 3.5 \mathrm{~m}$ & $\pm 101 \mathrm{~cm}$ & $\approx 2.2 \mathrm{~m}^{2}$ \\
PHENIX & - & Scintillators & $30-60 \mathrm{~m}$ & $\approx 3 \mathrm{~m}$ & - \\
BRAHMS & TOF Wall & Scintillators & - & $4.3 \mathrm{~m}^{*}(\max )$ & $\approx 0.21 \mathrm{~m}^{2}$ \\
PHOBOS & Paddle & Scintillators & $\pm 3.2 \mathrm{~m}$ & $23.1 \mathrm{~cm}$ & $\approx 0.17 \mathrm{~m}^{2}$ \\
\hline \multicolumn{7}{|c}{ The TOF Wall swings from $30^{\circ}$ to $90^{\circ}$ relative to the beamline. }
\end{tabular}

distant from the beam pipe than the ZDCs but closer to the interaction point. The STAR detector provided gated signals from their beam-beam counters (BBC) that are sensitive to particles traversing the detector in the direction of the blue and yellow ring but out of time with collisions, called the "blue halo" and "yellow halo" signals respectively. PHENIX provided signals from a number of scintillators in the tunnel, placed along the IR triplet, D0 and DX magnets, that were more sensitive to particles causing background in the MuID arms. The BRAHMS Time of Flight Wall (TOFW) was used to measure detector backgrounds. PHOBOS provided paddle detector rates and radiation monitors for background measurements.

The $\beta^{*}$ in all detectors was initially $2 \mathrm{~m}$ during the FY2003 deuteron-gold run. During the run, $\beta_{P H O B O S}^{*}$ was increased to $4 \mathrm{~m}$ to avoid beam scraping at the abort kickers in both rings. Later in the run, $\beta_{P H O B O S}^{*}$ and $\beta_{B R A H M S}^{*}$ were changed to $3 \mathrm{~m}$ to increase the luminosity in PHOBOS and reduce background in BRAHMS. Early in the FY2003 polarized proton run, there were collisions at all experiments. Later in the run only PHENIX and STAR had collisions. Removing two collision points reduced the total beam-beam tune shift which allows increased beam current and thus higher luminosity [28].

Figure 6.8 shows backgrounds at all of the RHIC experiments while the yellow scraper is being inserted during Fill 03094 in the deuteron-gold run with $\beta_{P H O B O S}^{*}=4 \mathrm{~m}$. 


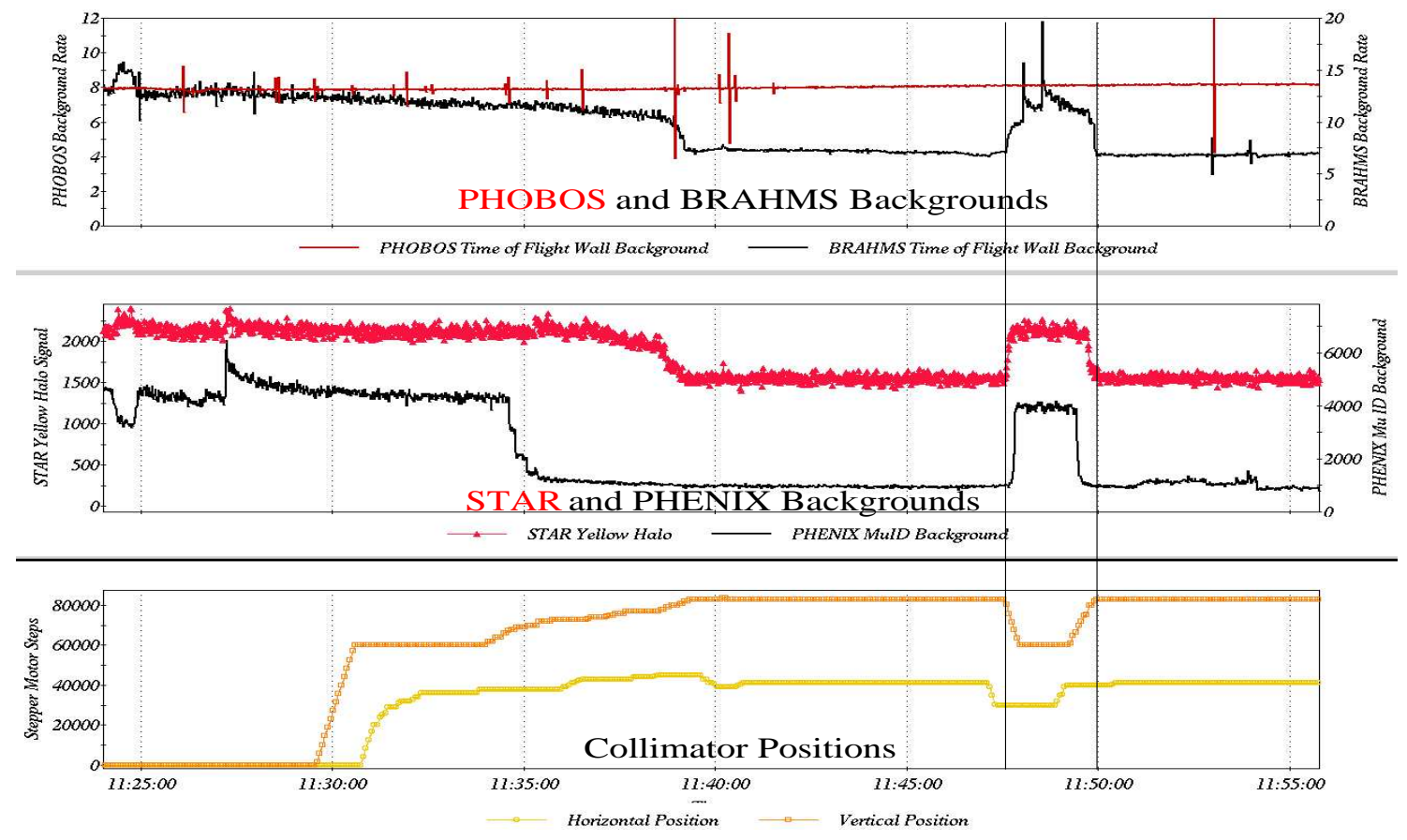

Figure 6.8: Experimental backgrounds during Fill 03094 in the FY2003 d-Au run. The bottom graph shows the yellow scraper horizontal and vertical position in stepper motor steps. Only PHOBOS does not benefit from scraping. The vertical scraper retraction clearly raises experiment backgrounds as indicated by the vertical lines.

The scraper reduces backgrounds in all detectors except PHOBOS. Similar results were achieved for the blue scraper. The PHOBOS background never benefited or was impaired by collimation. Table 6.4 shows the ratio of uncollimated to collimated background averaged over four stores for each configuration for the deuteron-gold and polarized proton runs in FY2003.

Figure 6.8 also shows the effectiveness of vertical collimation in the yellow ring, especially between 11:47:30 and 11:50. However, BRAHMS backgrounds were reduced most by yellow horizontal collimation. STAR blue background was removed with the horizontal scraper. The asymmetric beams during the d-Au run required that the beams pass through the interaction regions at an angle relative to the beam centerline. This decreased the available horizontal aperture at all the detectors. PHENIX always needed additional vertical collimation in both rings during this run. 
Table 6.4: Background reduction in FY2003 runs

\begin{tabular}{|c|c|c|c|c|c|}
\hline & \multicolumn{5}{|c|}{ Ratio of Uncollimated to Collimated background } \\
\hline Lattice & BRAHMS & PHENIX B & PHENIX Y & STAR B & STAR Y \\
\hline d-Au - $\beta^{*}=4 \mathrm{~m}$ & 3 & 2 & 4 & 1 & 2 \\
d-Au - $\beta^{*}=3 \mathrm{~m}$ & 3 & 4 & 2 & 5 & 1 \\
PP - all exp. & 1 & 3 & 23 & 4 & 1 \\
PP - two exp. & - & 11 & 22 & 16 & 2 \\
\hline
\end{tabular}

$\beta^{*}$ refers to $\beta_{P H O B O S}^{*}$. Prior to changing to $\beta_{P H O B O S}^{*}=4 \mathrm{~m}$, scrapers were not used.

Large backgrounds at PHOBOS reduced luminosity and backgrounds at all experiments.

STAR B and STAR Y refer to the STAR blue and yellow halo signals. PHENIX B and

PHENIX Y refers to scintillators in the tunnel downstream of the PHENIX detector near the blue and yellow ring respectively.

The reason is not completely understood. The large beam size limits the orbit adjustments that can be made. These adjustments had little effect on PHENIX backgrounds.

Localization of beam losses is another important function of the collimation system, especially in a superconducting machine such as RHIC. The stores used for profile measurements with the collimators are useful for studying the location of beam losses caused by collimation. Figure 6.9 shows the losses in the blue ring at injection during Fill 03254. The times when the collimator was moved are highlighted. The color scale indicates the loss rate in Rad/hr. The maximum of the color scale is set to $50 \mathrm{Rad} / \mathrm{hr}$ to maintain sensitivity to other losses around the ring.

The distance between the scraper and the beam pipe center changes from $-20.7 \mathrm{~mm}$ to $-8.25 \mathrm{~mm}$ from the bottom to the top of the figure. The losses at the collimator are between $2500 \mathrm{Rad} / \mathrm{hr}$ and $4700 \mathrm{Rad} / \mathrm{hr}$ for Figure 6.9, increasing with each movement from the bottom of the figure to the top as the scraper is inserted into the beam. Losses appear immediately downstream of the collimator in quadrupoles 4 and 5 (Q4 and Q5), dipoles 10 and 14 (D10 and D14) and the blue abort kicker. As mentioned above, the abort kickers are a known aperture limitation 


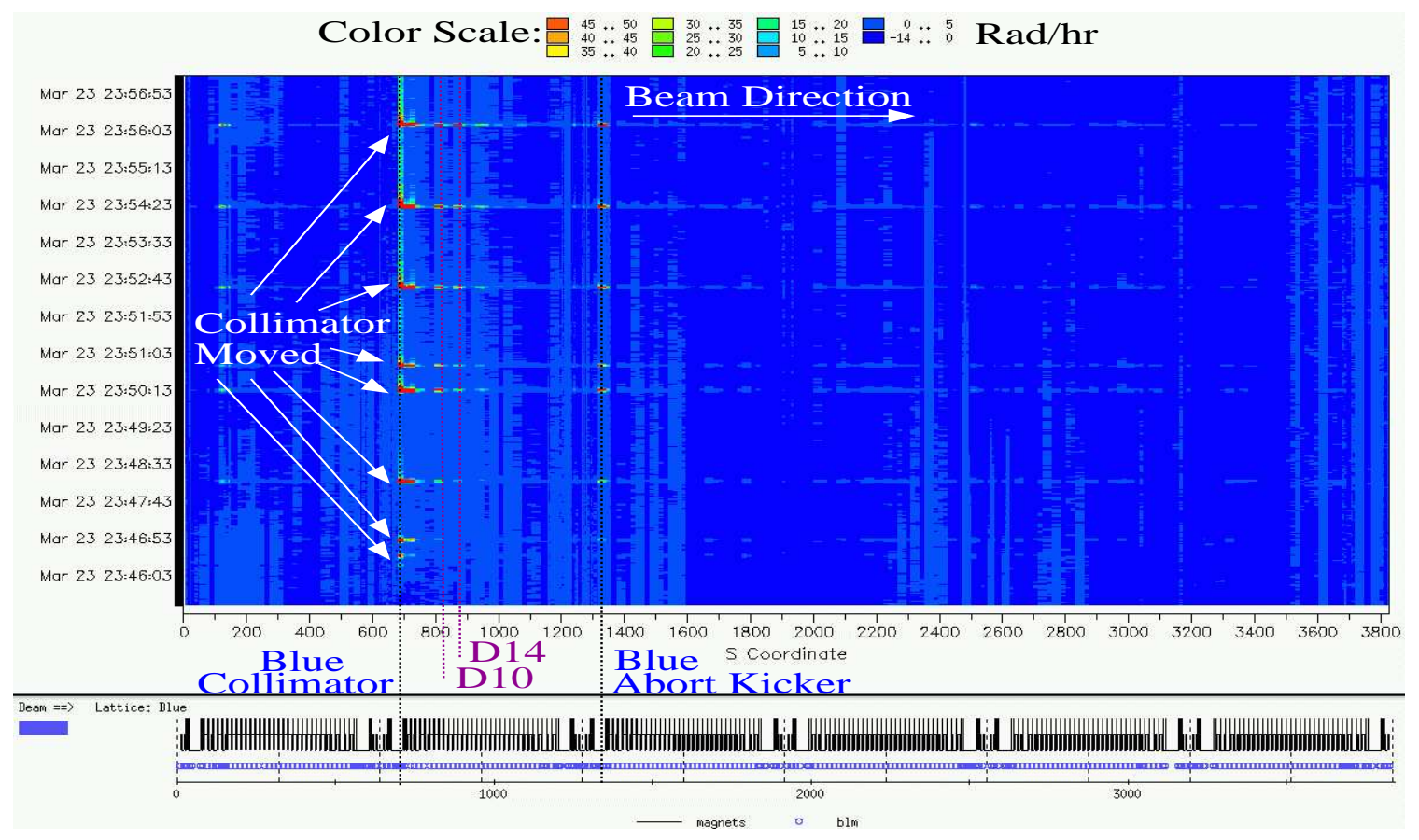

Figure 6.9: Loss pattern at injection for Fill 03254 in the blue ring. Losses appear at the collimator, quadrupoles 4 and 5, dipoles 10, 14, and 15, and the blue abort kicker.

in RHIC. Losses at Q4 and Q5 are direct spray from the collimator. Early simulations showed that the region around D10 is at an appropriate phase advance to place a secondary collimator [81]. The same study showed that this secondary collimator could also be used for a dispersion collimator since this region has a large dispersion. Dipole 14 also has a large dispersion.

Figure 6.10 shows a comparison of simulation, using ACCSIM, and measured beam losses during Figure 6.9. In this simulation, only magnet apertures are included since warm space apertures are unknown for this run. The simulation was also done with protons for reasons discussed in Section 4.1.3. The simulation shows losses at Q4, Q5, D9, and D10. The pattern does not match the beam losses, but the locations are similar. The abort kickers do not appear in the simulation because they are not included in the simulation. It is possible that the simulated loss at $s \approx 1525 \mathrm{~m}$ is from particles that should have hit the abort kickers. The losses at $s \approx 121 \mathrm{~m}$ are 


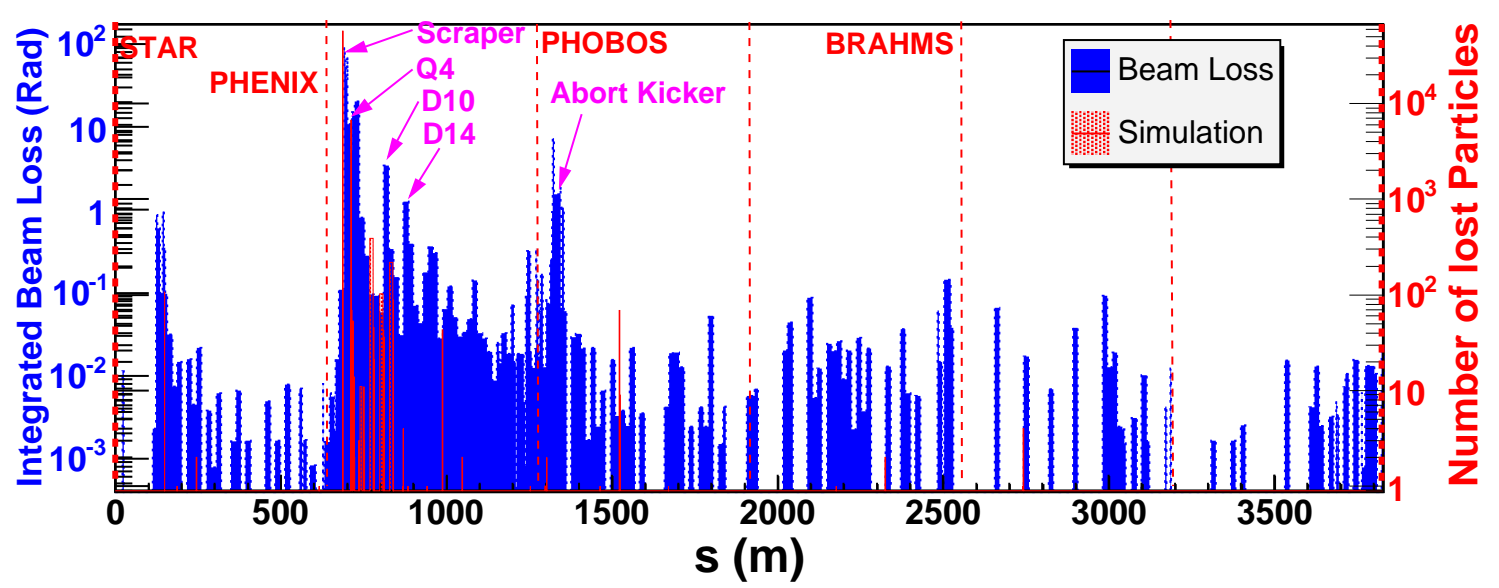

Figure 6.10: Comparison of integrated and simulated loss pattern at injection for Fill 03254 in the blue ring. Integration time is $20 \mathrm{~s}$. The horizontal scraper is $10 \mathrm{~mm}$ from the beam in the integrated losses and simulation. Vertical axes are log scale.

due to the injection kicker. This location is reproduced in the simulation.

The simulation also assumes that the beam orbit is the design orbit with design optics. As mentioned in Section 5.2, there are differences between the design optics and measured optics in the collimator region. This affects simulated loss locations, especially in the region of the abort kickers, since the optics in that region are similar to the collimator region.

Figure 6.11 shows a comparison of simulation to the measured beam losses in RHIC in Fill 03750 during the FY2003 polarized proton run. The data has the yellow scraper stationary in the beam. The blue scraper is being inserted into the beam during this time. The lattice used in the simulation does not have $\beta^{*}=10 \mathrm{~m}$ at PP2PP. Rather, all interaction regions have $\beta^{*}=2 \mathrm{~m}$.

The measured losses at $s \approx 600 \mathrm{~m}$ and $s \approx 1230 \mathrm{~m}$ are due to the yellow scraper. The losses in the data with $s>2200 \mathrm{~m}$ are not real with the exception of $s \approx 3200 \mathrm{~m}$. The pedestals for these BLMs are not optimally set. The losses at $s \approx 3200 \mathrm{~m}$ are due to scraping in the RF cavity. These losses appear only when the blue collimator 


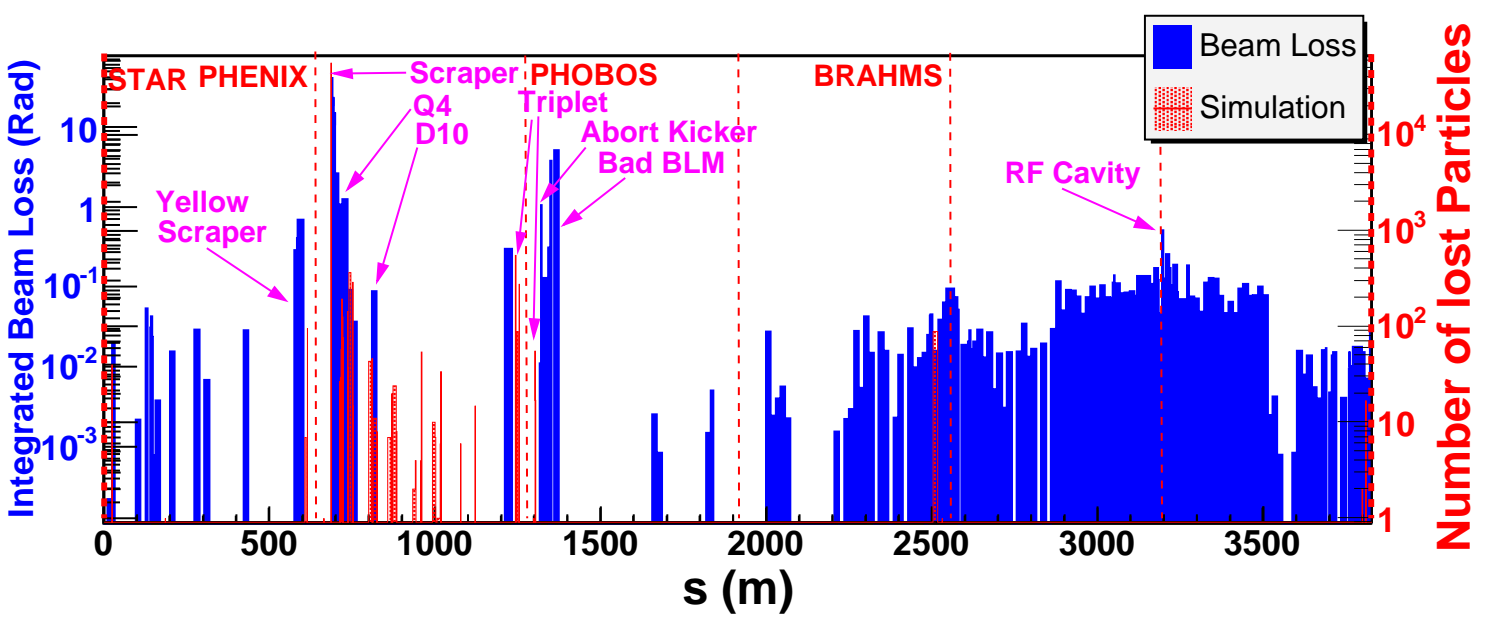

Figure 6.11: Comparison of integrated and simulated loss pattern at injection for Fill 03750 in the blue ring. Integration time is $20 \mathrm{~s}$. Vertical axes are log scale.

moves. The RF cavity apertures are not in the simulation.

The simulation shows losses that continue into the 8-9 o'clock arc that do not appear in the data. The data show larger losses than the simulation at Q4. However, both show losses at D10. The simulation shows losses in the triplet magnets for PHOBOS instead of at the abort kicker. The simulation result is not surprising because the triplet magnets can be an aperture restriction.

Another way to compare the simulations to the data is to define a scattering reduction factor for the simulation as

$$
\epsilon=\frac{\text { total lost particles }}{\text { total lost particles }- \text { particles lost at scraper }}
$$

which is always greater than unity, and compare it to the background reduction seen in Table 6.4. Even though these numbers are not directly comparable, extreme differences between these numbers would show that we have a gross misunderstanding of the collimation system. 
Averaging the simulations in Figures 6.10 and 6.11 yields $\epsilon=8.2 \pm 0.5$. The average background reduction for all experiments during both runs is $5.8 \pm 0.2$. Protons are used in the simulation. The average background reduction using only the proton data is $9.2 \pm 0.3$, within two standard deviations of the simulation result.

We hope that adding the warm space apertures will increase the accuracy of the simulations, not only by predicting the collimation efficiency, but also the loss pattern. Simulations of the collimation system upgrade using a more complete aperture list are discussed in the next chapter.

\subsection{Longitudinal Halo Removal}

During the length of the store, particles can migrate out of the RF bucket, as discussed in Section 1.3. These particles drift longitudinally in RHIC and eventually fill the space between bunches. Some fraction of this beam will miss the beam dump when the beam is ejected. This beam does not receive the full kick from the abort kickers because it fills the location in the bunch train left empty for the kicker rise time, called the abort gap.

This coasting beam is removed with a procedure named "gap cleaning", which has two parts. First, the beam in the abort gap is excited transversely using the RHIC tunemeter kicker. Then the scrapers are used to intercept this beam to clean the abort gap [82].

Figure 6.12 shows the relative positions of the tunemeter kickers and the scrapers in RHIC. Each ring has two kicker modules with two $2 \mathrm{~m}$-long stainless steel striplines

allowing both horizontal and vertical kicks. All striplines in both rings are charged by one power supply producing a pulse approximately $140 \mathrm{~ns}$ long. The tunemeter kicker is described more fully in Reference [83]. 


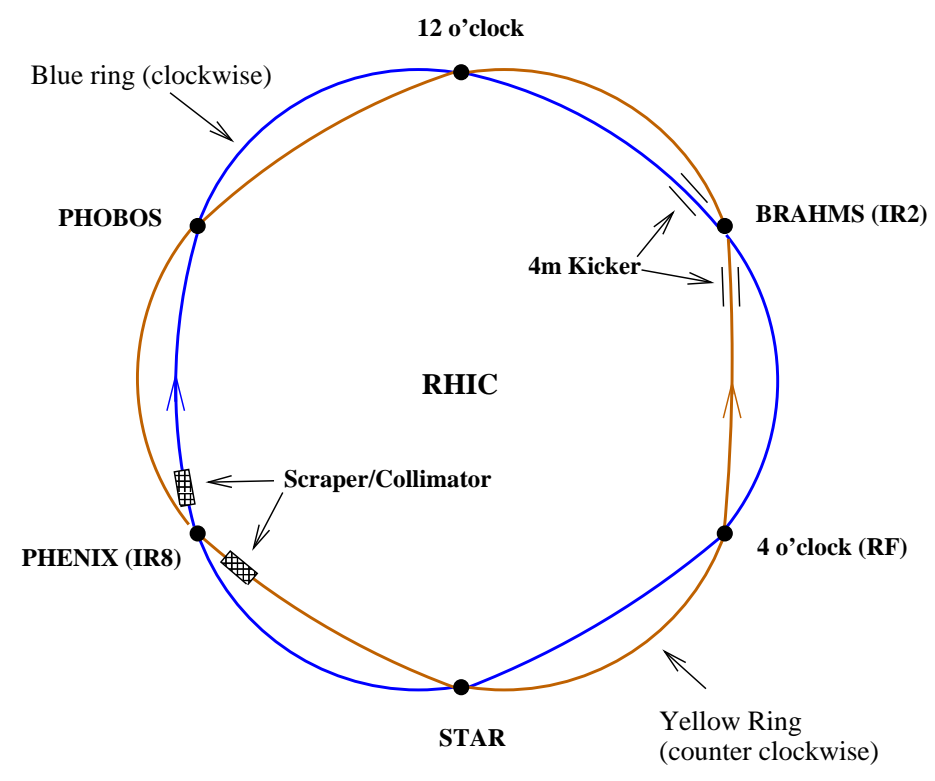

Figure 6.12: Location of the tunemeter kickers and scrapers in RHIC

The kicks are timed to be in the abort gap with its buckets numbered 331-360. Typically 300 turns per trigger are used. Prior to the FY2003 run, these triggers were repeated every four seconds. For the FY2003 run, the trigger frequency was one Hertz.

Choosing the proper kick frequency is critical to exciting the beam. The kick frequency is chosen to be close to the betatron frequency in both planes. A frequency equal to or very close to the betatron frequency was shown to kick bunched beam at storage energy out of the ring after a few dozen turns. The frequency was scanned from 0.2 to 0.25 in steps of 0.01 tune units. To determine the optimal frequency, one could either measure the tunes of the debunched beam by using the tune meter or monitor the beam loss at the scrapers while the excitation frequency was scanned. Measuring the tunes in the abort gap with the tunemeter is only possible when there is a large amount of debunched beam. Typically, a resonant frequency could be found in the horizontal plane only due to the favorable horizontal $\beta$-function at the location of the kicker. 
The collimators were placed in a predefined transverse position at the start of the procedure. Once the tunemeter started kicking, the collimator positions were adjusted to maximize the cleaning efficiency. Typically, the collimators were inserted as far as possible without affecting the bunched beam lifetime, and were adjusted during the procedure if necessary.

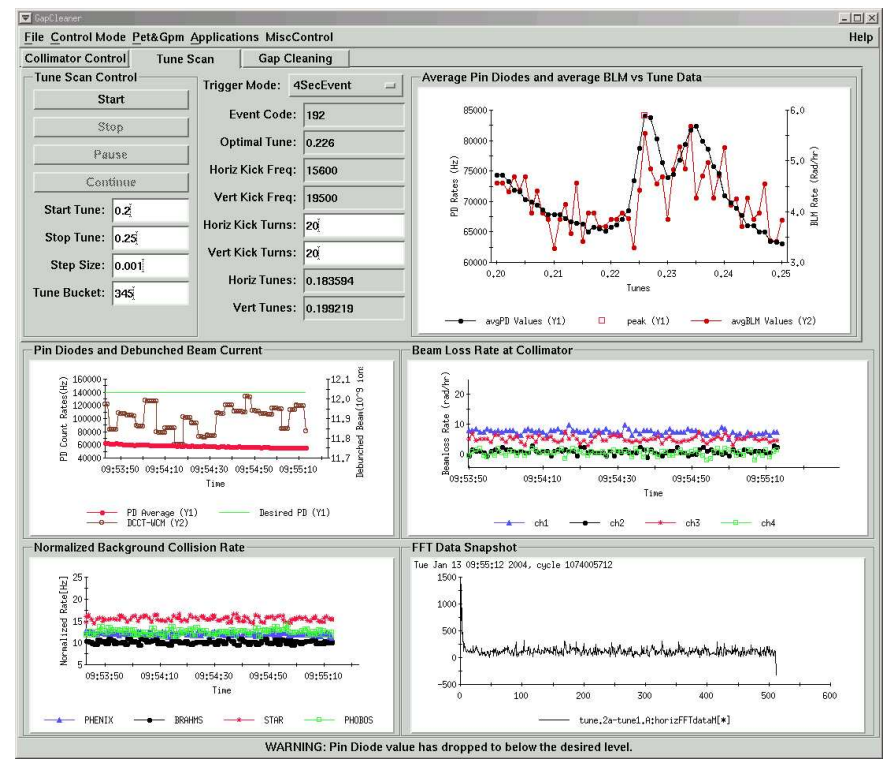

Figure 6.13: The GapCleaner program.

Prior to the FY2003 run, these steps were done manually. For the FY2003 run, these steps were partially automated with the help of an application, GapCleaner [84]. Figure 6.13 shows a screen shot of the application. The application automates the tune scan. It has options to adjust the tunemeter kick frequency and timing in the abort gap. GapCleaner can open the Collimator application for collimator control during gap cleaning.

A dedicated kicker and electronics were installed for the FY2004 run to further increase gap cleaning efficiency. Problems with the pulser did not allow its use, so the tunemeter was used again. In this run, the tunemeter kicked during the entire store at the bunched beam tune frequency. This does not allow debunched beam to 


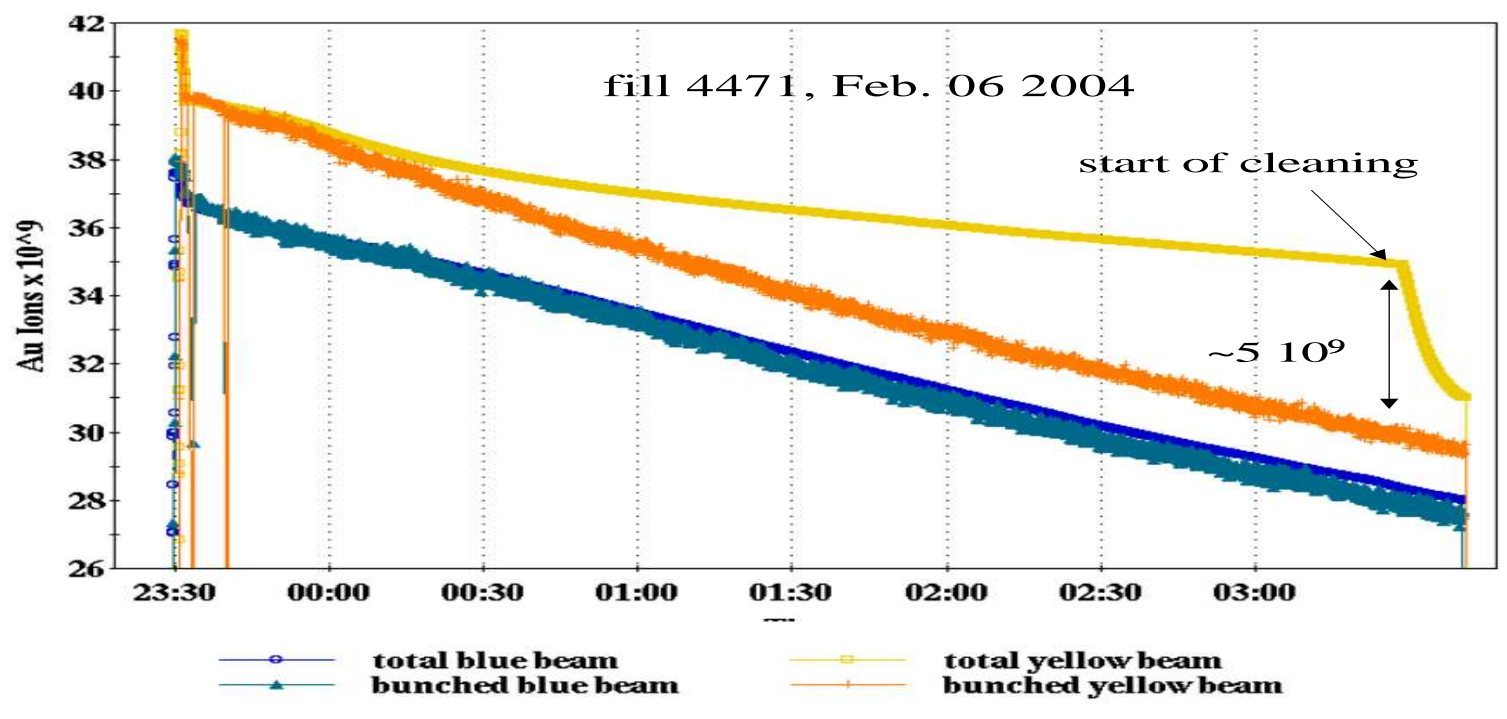

Figure 6.14: Gap Cleaning during Fill 04471

build up and reduces the risk of magnet quenches.

Figure 6.14 shows gap cleaning during Fill 04471. Continuous gap cleaning occurs in the blue ring. Gap cleaning is done in the yellow ring only at the end of the store when the debunched beam in the yellow ring exceeds $5 \times 10^{9}$ ions. The amount of debunched beam is determined from the difference between the DCCT and the Wall Current Monitor [85]. Approximately $4 \times 10^{9}$ ions are removed in approximately 11 minutes. Further discussion of gap cleaning can be found in References [82, 86].

\subsection{Diffusion Studies}

In Section 1.3 various mechanisms are discussed that cause beam halo growth. One way to measure the halo growth rate is using a collimator to measure the diffusion rate of the beam. To reconstruct the diffusion coefficient as a function of the particle action, the loss rate at a collimator is measured after it moves relative to the beam. The theory of how to measure beam diffusion with a collimator is treated in detail 
in References [33, 87]. The following treatment closely follows Reference [87].

The diffusion equation is

$$
\frac{\partial}{\partial t} f(J, t)=\frac{1}{2} \frac{\partial}{\partial J} B(J) \frac{\partial}{\partial J} f(J, t)
$$

where $f(J, t)$ is the beam distribution as a function of the particle action $J$ and time, and $B(J)$, given by

$$
B(J)=\frac{<\Delta J^{2}>}{\Delta t} \approx b J^{n}
$$

is the diffusion coefficient to be measured. $B(J)$ is generally postulated to be a monomial. If it is assumed that only particles that are initially close to the collimator will eventually hit the collimator, then near the collimator $B(J)$ can be written,

$$
B(J)=b_{0}\left(\frac{J}{J_{c}}\right)^{n}
$$

where $J_{c}$ is the action of a particle that just touches the collimator,

$$
J_{c}=x_{c} / \sqrt{2 \beta}
$$

where $x_{c}$ is the distance between the collimator and the beam center, and $\beta$ is the $\beta$ function at the collimator. The diffusion coefficient at $J=J_{c}$ is $b_{0}=b J_{c}{ }^{n}$.

Assuming that $f\left(J_{c}, t\right)$ vanishes at the collimator, and that $f(J, 0)$ increases linearly away from the collimator, then the left hand side of Equation. 6.11 is just the particle loss rate due to the collimator, $\dot{N}(t)$. To solve Equation. 6.11 for the loss 
rate at the collimator, it is useful to introduce the variables,

$$
\begin{aligned}
z & =\frac{J_{c}-J}{J_{c}} \\
R & =\frac{b_{0}}{2 J_{c}{ }^{2}}
\end{aligned}
$$

which respectively are the fractional change in the collimator action, and the normalized diffusion rate to be determined by a fit. For the instance when the collimator moves toward or away from the beam, one obtains:

$$
\begin{aligned}
& \dot{N}^{(1)}(t)=a_{0}\left\{1+\frac{\Delta z}{\sqrt{\pi R\left(t-t_{0}\right)}}\right\}+a_{1} \\
& \dot{N}^{(2)}(t)=a_{0} \operatorname{erfc}\left[\frac{\Delta z}{\sqrt{4 R\left(t-t_{0}\right)}}\right]+a_{1}
\end{aligned}
$$

where $\Delta z=2\left|\Delta x_{c}\right| / x_{c}$ is the absolute change in $z$ due to the change in collimator position $\Delta x_{c}, a_{1}$ is the count rate of the PIN diodes with the collimator fully retracted, and $a_{0}$ is an arbitrary constant. By fitting one of these solutions to the loss rate after moving the collimator, it is possible to obtain the normalized diffusion coefficient, $R$, at each collimator action. The diffusion coefficient at the collimator action is reconstructed with

$$
B\left(J_{c}\right)=2 J_{c}^{2} R
$$

By sampling over many collimator positions, it is possible to reconstruct the diffusion coefficient for the beam halo.

Data were taken during all RHIC runs, in both rings, with all species, at storage energy during luminosity production. Dedicated studies were necessary for injection energy studies. For each type of movement of the collimator, the loss rate is fit to either of Equations 6.16a or 6.16b, as shown in Figure 6.15. 

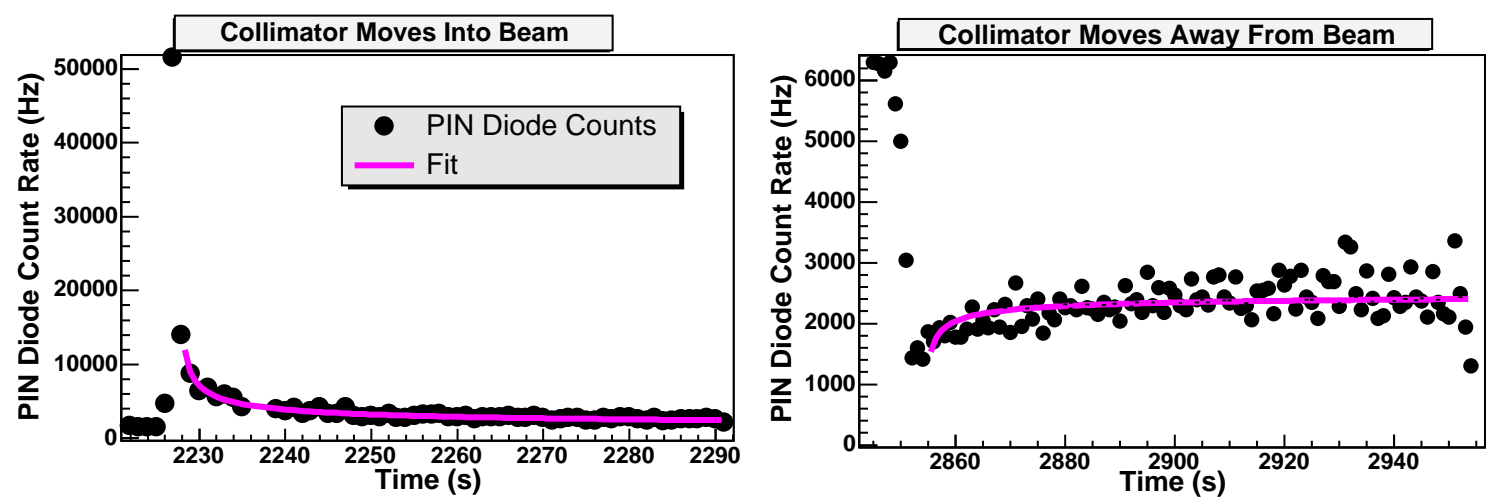

Figure 6.15: The effect of the collimator motion on beam loss rates with fit. The scraper is inserted into the beam in the left figure. It is retracted in the right figure.

The normalized diffusion coefficient, $\mathrm{R}$, is averaged over the four PIN diodes. This value is used to reconstruct $B\left(J_{c}\right)$ and the results are fit to Equation 6.12. Figure 6.16 shows the reconstruction of the $B(J)$ for Fill 02797 . The horizontal and vertical error bars are dominated by the uncertainty in the collimator action. This uncertainty is equally due to the uncertainty in the distance between the collimator and the beam and the knowledge of the $\beta$ function at the collimator [72].

Table 6.5 shows the results of the fits for all runs. Four data sets were taken at injection energy. For Fill 03155, two data sets were taken, the first immediately after the ramp to storage energy. The second was taken two and a half hours later.

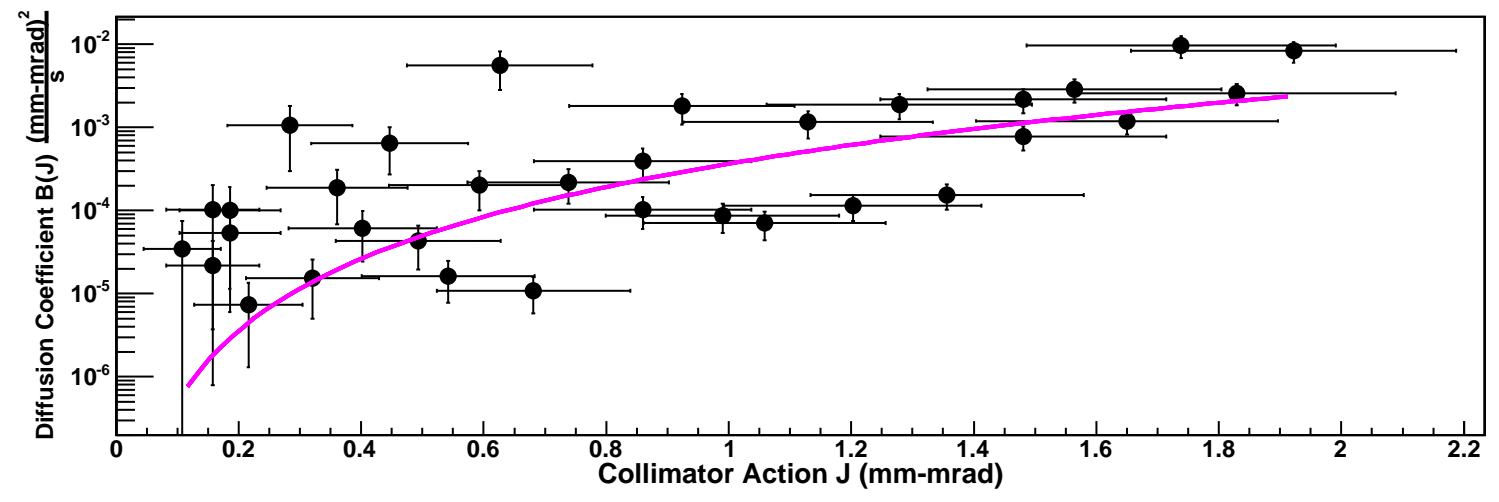

Figure 6.16: Reconstructed diffusion coefficient for Fill 02797 in the yellow ring. Note the vertical axis has a log scale. 
Table 6.5: Results of fit to $B(J)=b J^{n}$

\begin{tabular}{|c|c|c|c|c|c|}
\hline Store Number & Year & Ring & Beam & $b \mu \mathrm{m}^{2-n} \mathrm{~s}^{-1}$ & $n$ \\
\hline 00854 & 2001 & yellow & $\mathrm{Au}$ & - & - \\
01413 & 2001 & yellow & $\mathrm{Au}$ & $0.17 \pm 0.09$ & $10.3 \pm 1.2$ \\
\hline $01874(\mathrm{i})$ & 2002 & yellow & $\mathrm{p}$ & $0.05 \pm 0.03$ & $8.5 \pm 1.5$ \\
$01924(\mathrm{i})$ & 2002 & blue & $\mathrm{p}$ & $0.06 \pm 0.02$ & $7.0 \pm 0.8$ \\
02135 & 2002 & yellow & $\mathrm{p}$ & $240 \pm 670$ & $14 \pm 4$ \\
02136 & 2002 & yellow & $\mathrm{p}$ & $8 \pm 6$ & $5.7 \pm 0.6$ \\
02175 & 2002 & blue & $\mathrm{p}$ & $0.0036 \pm 0.0005$ & $3.0 \pm 0.3$ \\
\hline $02797(\mathrm{i})$ & 2003 & blue & $\mathrm{d}$ & $(1 \pm 9) \times 10^{-9}$ & $19 \pm 9$ \\
$02797(\mathrm{i})$ & 2003 & yellow & $\mathrm{Au}$ & $(3.6 \pm 0.8) \times 10^{-4}$ & $2.8 \pm 0.3$ \\
02959 & 2003 & yellow & $\mathrm{Au}$ & $0.0081 \pm 0.0014$ & $8.3 \pm 0.8$ \\
$03155-01(\mathrm{v})$ & 2003 & yellow & $\mathrm{Au}$ & $(5 \pm 4) \times 10^{-4}$ & $3.5 \pm 1.0$ \\
$03155-02(\mathrm{v})$ & 2003 & yellow & $\mathrm{Au}$ & $2 \pm 1$ & $8.7 \pm 0.8$ \\
\hline
\end{tabular}

(i) indicates injection energy. (v) indicates vertical plane.

For this fill, the diffusion measurement was done in the vertical plane because of an oscillation of the beam orbit caused by the Booster/AGS cycle seen in the horizontal plane, discussed in Section 6.6. All other measurements were done in the horizontal plane.

The measurement for Fill 00854 yields a strange result. The reconstruction of $B(J)$ is different for the data fit to Equation $6.16 \mathrm{a}$ and $6.16 \mathrm{~b}$. This is unphysical and the reason for this is not understood. This did not happen for other datasets.

The results for Fill 02135 are also not understood. The data were taken at the start of the store. During acceleration, the horizontal tunes came close to the 0.2 resonance. This may be a possible cause of the puzzling results.

The BPM downstream of the collimator used to interpolate the beam position was missing during Fill 02797 in the blue ring. To compensate, a different BPM was used. However, there is a horizontally defocussing quadrupole in between the scraper and the BPM. The data were also taken at larger actions. These combine to give a larger uncertainty in the horizontal action. This is the reason for the very large uncertainty in $b$ and $n$. 
Using the remaining data, the mean value of the exponent $\langle n\rangle=4.2 \pm 0.2$. There seems to be little correlation between $b$ and species or ring. Before the data was taken in Fill 02136, a van der Meer scan was done in the PHENIX IR [88], and before the second data set in Fill 03155, orbit bumps were performed through the same IR [89]. Both of these measurements have a large $b$ as compared to all other measurements. In these cases, the beam passes off center through the IR triplet magnets and samples nonlinear magnetic fields. This may cause the relatively large $b$ in these cases.

The reconstructed diffusion coefficients for Fills 01874 and 01924 are consistent. These sets were taken at injection energy with protons, however the rings are different. It seems that $B(J)$ at injection is determined largely by the species and energy of the beams. The diffusion coefficient for Fill 02797 in the yellow ring cannot be compared to Fills 01874 and 01924 because the injection energy for gold is lower than that of protons, and is also below transition energy.

\subsection{Frequency Analysis of Beam Losses}

A frequency analysis of the PIN diodes is useful in revealing slow beam motion. By performing a Fast Fourier Transform (FFT) on the PIN diode rate it is possible to extract the frequencies of these motions. This can be a valuable method of detecting additional ways of beam loss that might not be accessible with other methods.

The PIN diode rates are sampled at $1 \mathrm{~Hz}$, therefore the FFT is only sensitive to frequencies less than $0.5 \mathrm{~Hz}[90]$. By sampling at a faster rate, it is possible to measure higher frequencies. It was not possible to sample with frequencies greater than $10 \mathrm{~Hz}$ due to limits of the FEC.

Figure 6.17 shows a portion of a PIN diode time series for Fill 01020 in the blue 

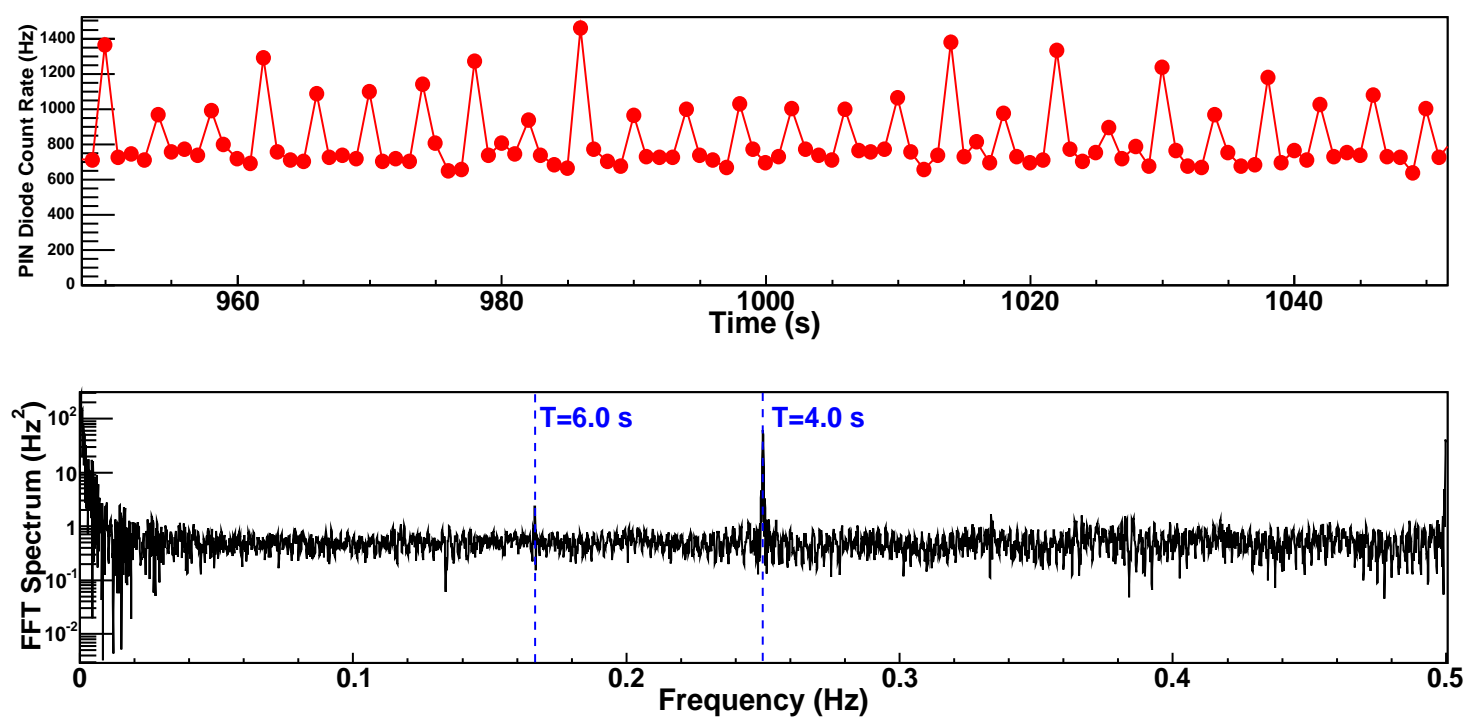

Figure 6.17: Blue PIN diode rate and FFT during Fill 01020. The top graph shows the PIN diode rate vs. time. The lower graph shows the FFT of the PIN diode data. The two peaks in the FFT spectrum are the Booster/AGS supercycle and the tunemeter kicker.

ring. At this time the scraper was inserted into the beam and the RHIC tunemeter was kicking one bunch every four seconds. The effect of the tunemeter is clearly seen in the time series. A FFT of the time series clearly shows a spike at $0.25 \mathrm{~Hz}$. In addition there is a spike at $0.1665 \mathrm{~Hz}$. This corresponds to the frequency of the Booster/AGS supercycle at the time. Each Booster/AGS supercycle contains a number of Booster cycles to inject into AGS and one AGS cycle.

It was known that the RHIC Phase Locked Loop Tunemeter measured tune changes with each Booster cycle [91]. However, it was unexpected that the collimation system would be sensitive to this effect. The supercycle was temporarily stopped to confirm that the Booster/AGS supercycle was responsible for this effect. Figure 6.18 shows the results of this experiment, clearly showing spikes at the supercycle period, $4.8 \mathrm{~s}$ in this case. When the supercycle is stopped, the spikes go away. Because it was initially assumed that the Booster Main Magnet Power supply was the cause of this oscillation, the spikes associated with it are called "Booster spikes". 


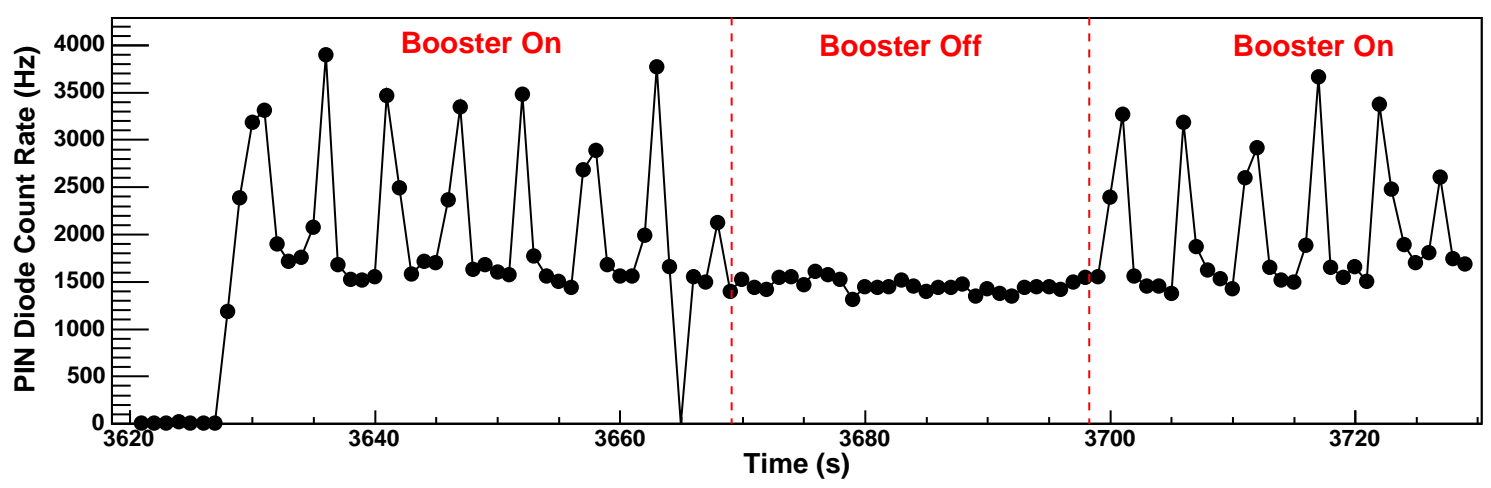

Figure 6.18: Effect of the Booster/AGS supercycle on the PIN diode signal in the yellow ring. The peaks disappear when the Booster is turned off.

The next investigation was to run the supercycle and turn off individual power supplies one at a time to determine the source. The Booster Main Magnet Power Supply was turned off first. The spikes did not disappear. Because of time constraints, it was not possible to turn off more power supplies, therefore the ultimate cause of the Booster spikes is unknown.

Figure 6.19 shows the PIN diode rates while the yellow vertical scraper is scrap-
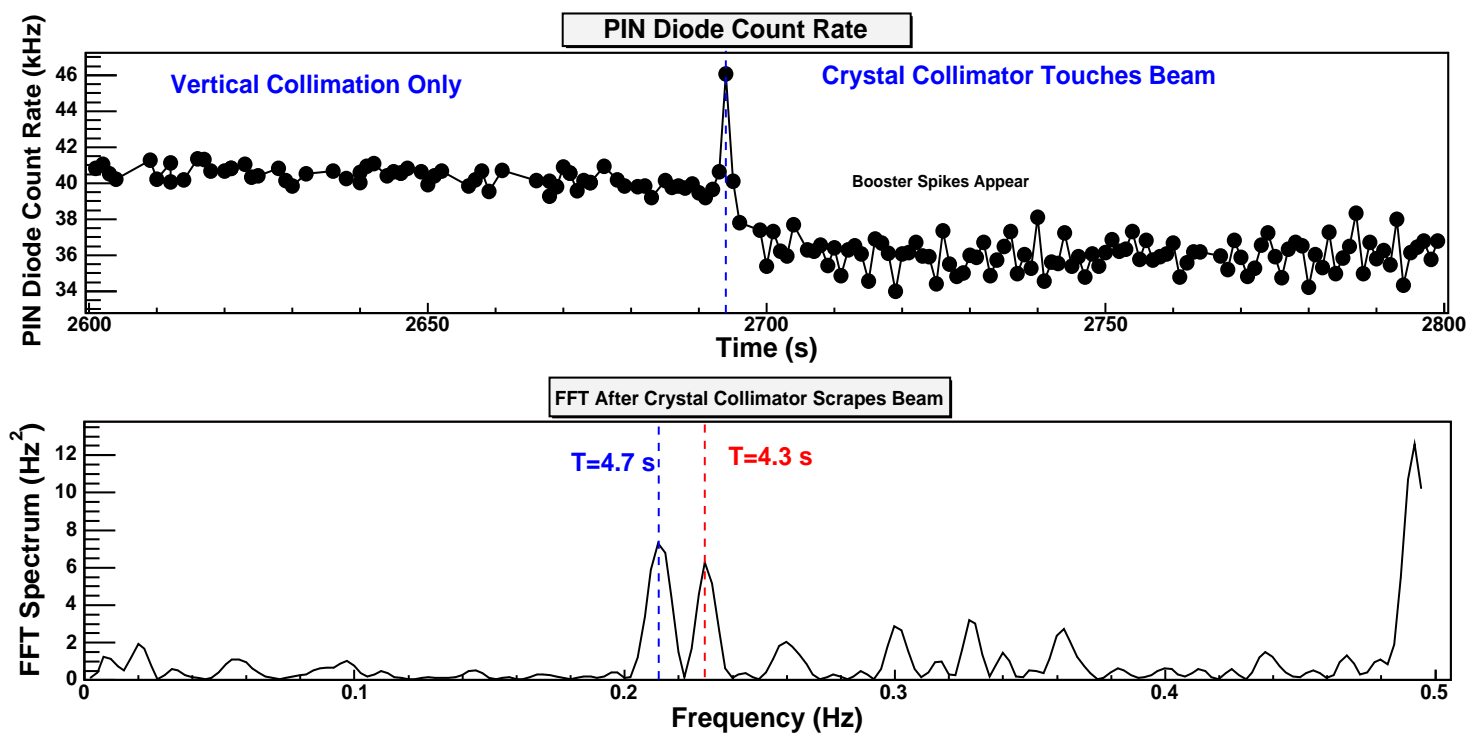

Figure 6.19: Pin diode rates before and after the crystal collimator touches the beam. The FFT peak at $4.3 \mathrm{~s}$ is not understood. The $4.7 \mathrm{~s}$ peak is the supercycle period. 
ing the beam. The Booster spikes are not present. However, when the crystal collimator was moved into the beam, the spikes appear. From this, it is conjectured that the Booster spikes cause an oscillation in the RHIC dipole bus. If the spikes were to also appear with vertical collimation, the quadrupole bus might be suspected as well. It was decided that when Booster spikes became a problem for data taking in other studies, the Booster/AGS supercycle would be turned off if possible.

The RHIC collimators were successful in removing halo and reducing experimental backgrounds. The collimators were used to measure beam profiles. Measurements of beam diffusion rates were performed. Slow oscillations of the beam were discovered. Attempts to calibrate the PIN diode rates to beam current losses are ambiguous.

The system was upgraded for FY2004 in response to the unsatisfactory performance of the crystal collimator and increased luminosity in the run. This is discussed in the next chapter. 


\section{Chapter 7}

\section{Upgrade of the RHIC Collimation System}

The unsatisfactory performance of the crystal collimator determined that it could not be used effectively in operations. Background rates during the deuterongold run required routine collimation. The lack of secondary collimators became a concern, especially for the high luminosity FY2004 gold-gold run. This chapter discusses the design of the collimation system upgrade and its performance during the FY2004 run.

\subsection{Design}

As discussed in Section 2.2.1, the optimal phase advance between the primary and secondary collimators is

$$
\Delta \phi_{\text {opt }}=m \pi \pm 20^{\circ} \ldots 30^{\circ} .
$$




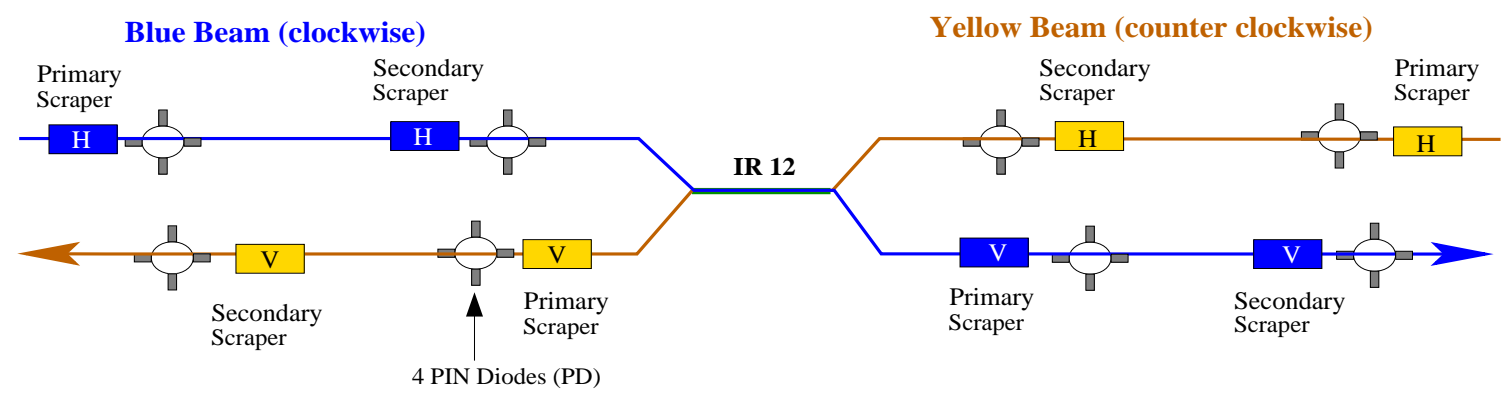

Figure 7.1: Suggested Collimation scheme placing collimators on either side of an interaction region. Installation of a waterproof liner makes this scheme too expensive.

If the primary collimators were to remain in their original positions, using this phase advance meant placing secondary collimators in the cold regions of RHIC. Time and financial constraints made this impossible. Schemes using the warm space around IP4 and IP12 shown in Figure 7.1 attempt to optimize the phase advance at the expense of having secondary halo propagate through an interaction region. The possibility of having spray from the scrapers directed at the triplet magnets is a definite disadvantage. This scheme was ultimately rejected because these interaction regions contain equipment that is sensitive to beam loss. IR4 is the location of the RF system, and IR12 is the location of the proton polarimeters. IR12 is also being considered for the eRHIC upgrade. Environmental considerations in the IR12 region also prohibited placing collimators there.

Long Island has a high water table. Groundwater contamination due to ${ }^{22} \mathrm{Na}$ and ${ }^{3} \mathrm{He}$ produced by neutrons escaping the RHIC tunnel is a concern. Brookhaven National Laboratory maintains a self imposed limit of $20 \mathrm{pC} / \mathrm{L}$ for ${ }^{22} \mathrm{Na}$ and $1000 \mathrm{pC} / \mathrm{L}$ for ${ }^{3} \mathrm{He}$. This is $5 \%$ of the local drinking water standard. A waterproof liner is placed around the tunnel in areas of RHIC were this standard may be exceeded. The liner prevents rain water from washing contaminants from the soil into the ground water. This liner also prevents ground water from rising into the contaminated soil. A liner is installed around the beam dumps and the original collimator section. 


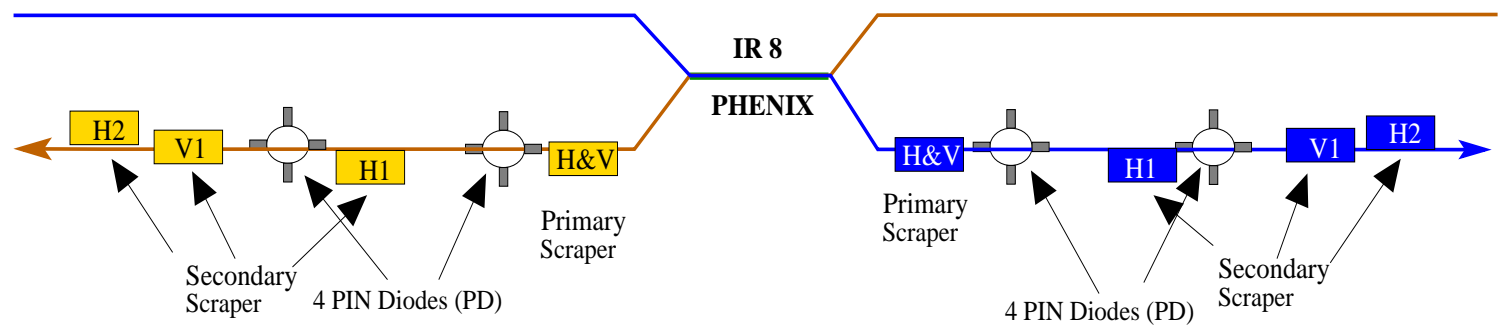

Figure 7.2: Upgraded RHIC Collimation System. PIN diodes downstream of collimators v1 and h2 are not shown for clarity. The inside of RHIC is toward the bottom of the figure.

The simplest option was to keep the primary collimators in the warm section downstream of PHENIX in both rings and to place secondary collimators in this section. Figure 7.2 shows the chosen collimator system upgrade. The existing copper scrapers are located immediately downstream of the triplet cryostat in the place that was reserved for the crystal collimator. A horizontal scraper (h1) is placed $10 \mathrm{~m}$ downstream of the primary. A vertical scraper (v1) is placed $16 \mathrm{~m}$ downstream of the primary with another horizontal scraper (h2) immediately downstream. The positioning of the secondary scrapers is discussed in the next section. An array of four PIN diodes is downstream of each scraper. Table 7.1 lists the design phase advance between the primary and secondary collimators. These phase advances are not optimal, however the extended drift allows sufficient separation of the scatter particles from the beam core.

The horizontal scrapers are similar to the primary collimator, discussed in Sec-

Table 7.1: Approximate phase advance between primary and secondary collimators

\begin{tabular}{|c|c|c|}
\hline Collimator & $\begin{array}{c}\text { Distance from } \\
\text { Primary Collimator }\end{array}$ & $\Delta \phi$ \\
\hline First Horizontal (h1) & $10 \mathrm{~m}$ & $0.7^{\circ}$ \\
First Vertical (v1) & $16 \mathrm{~m}$ & $6.8^{\circ}$ \\
Second Horizontal (h2) & $17 \mathrm{~m}$ & $1.5^{\circ}$ \\
\hline
\end{tabular}



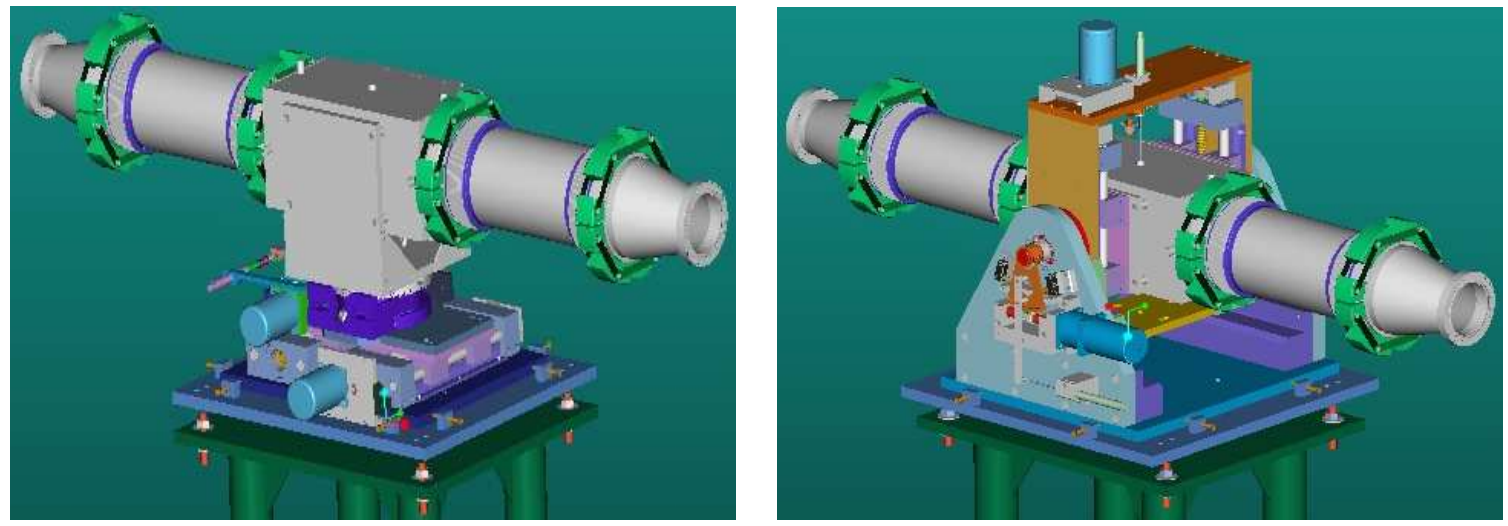

Figure 7.3: Horizontal (left) and vertical (right) secondary collimator vessels.

tion 3.1.4, without the vertical jaw and vertical motion. The first secondary scraper is located on the inside of the ring with the primary scraper, the second horizontal scraper approaches from the outside of the ring.

The vertical scraper vessel is shown in Figure 7.3. The original vessel was modified such that it moves vertically and rotates about the horizontal axis. The jaw approaches the beam from above just as the primary scraper. There is no secondary vertical scraper approaching from the bottom.

In addition to the changes in the collimation system, the PHENIX and BRAHMS detectors installed shielding in the RHIC tunnel. The goal of this shielding is to reduce the uncollimated background rate so that each detector can turn on without necessarily waiting for the collimators to be positioned. This uncollimated background comes from particle flux outside of the beam pipe due to scraping.

\subsection{Simulation}

To optimize the location of the scrapers in the warm section, simulations using K2 were done. The simulations assume the primary scraper is used at $6 \sigma_{x, y}$ and $7 \sigma_{x, y}$ in the horizontal and vertical planes. Positions on this order were used in previous 


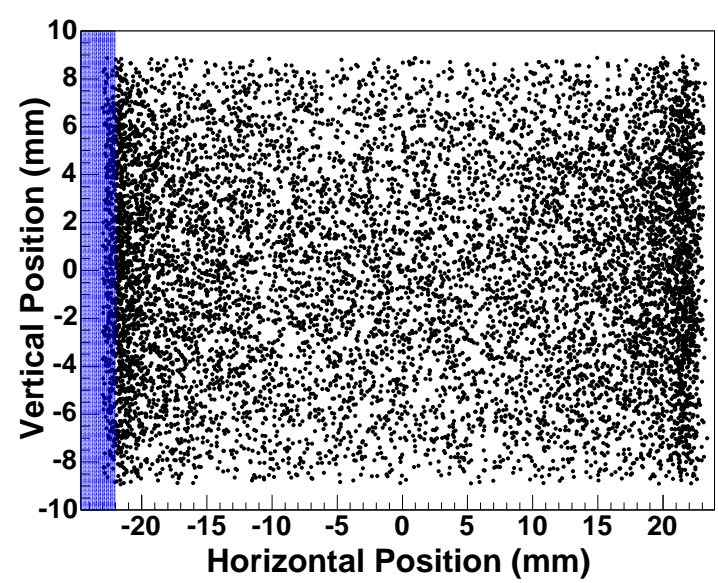

Figure 7.4: Input distribution used in simulations. The shaded area indicates collimator location, placed at $x=-6 \sigma_{x}$

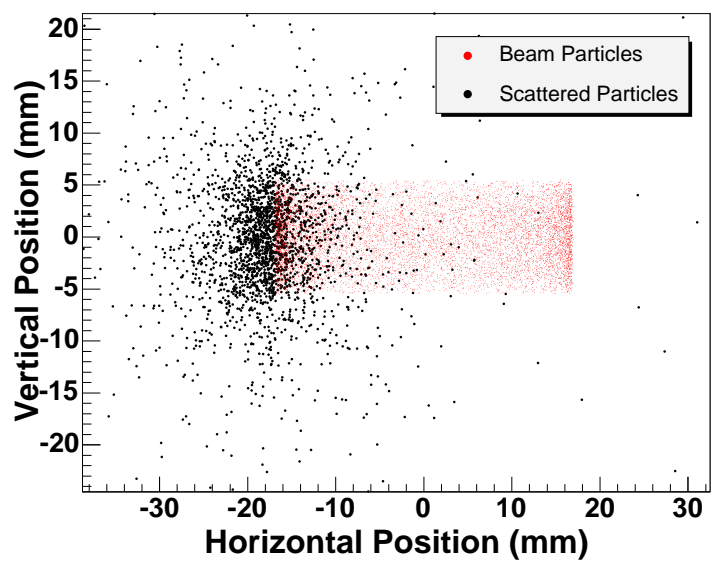

Figure 7.5: A simulation of scattered and beam particles $10 \mathrm{~m}$ downstream of the primary scraper.

runs. The particles emerging from the scraper are propagated downstream to the potential location of the secondary collimator. The scraper locations were chosen to optimize the number of intercepted particles.

Figure 7.4 shows an $x-y$ projection of the particle distribution used to simulate secondary halo at the location of the secondary collimators. The distribution is identical to all other particle distributions considered in this thesis. The primary collimator is inserted to scrape at $-6 \sigma_{x}$, as shown by the blue shaded region. Figure 7.5 shows the output of the simulation. The red dots are the particles in the beam core, the black are all of the particles scattered from the scraper after 20 turns. Figure 7.5 immediately shows that it is possible to place the first horizontal secondary close to the primary scraper because of the large scatter of particles on the left of the figure. It also shows that it is desirable to locate another horizontal secondary scraper and a vertical secondary scraper further downstream to intercept scattered particles that overlap with the beam core at the first horizontal collimator.

The position of the second horizontal secondary scraper was chosen to place the greatest possible distance between it and the primary scraper while remaining 


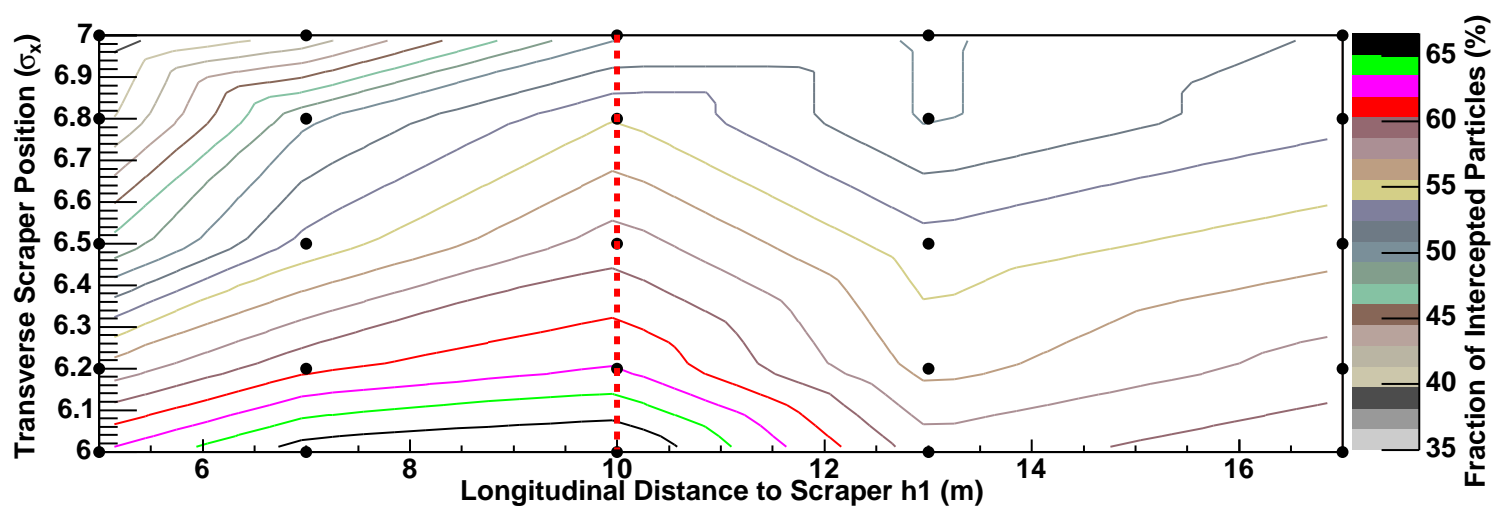

Figure 7.6: Simulation of the fraction of intercepted particles scattered from the primary scraper as a function of the secondary scraper transverse and longitudinal position. The points represent the simulated locations. The red dotted line shows the location of the h1 collimator.

in the warm region. This maximizes the number of particles that can drift from the horizontal primary scraper. The location of the first horizontal and vertical secondary scraper was chosen by taking the ratio of intercepted particles to the scattered particles at different distances and transverse positions locations. Figure 7.6 shows the fraction of intercepted particles as a function of distance and transverse position locations. The primary scraper is located at $6 \sigma_{x}$ and is retracted vertically. Each point represents the positions that were simulated. The red dotted line indicates the chosen longitudinal position for the first horizontal secondary.

Figure 7.6 shows that a horizontal secondary collimator will intercept the most particles when it is placed at $x \approx 6 \sigma_{x}$ and falls off as the scraper is retracted. A maximum exists at $s \approx 10 \mathrm{~m}$, regardless of the transverse position. The reason for this maximum is not understood. As a result of these simulations, the first horizontal secondary is located $10 \mathrm{~m}$ downstream of the primary scraper.

Similar simulations show that the vertical secondary scraper should be located as far as possible from the vertical primary scraper. The $\beta$ function changes slowly and requires a larger drift to separate the scattered particles from the beam core. 
For this reason, it was placed immediately upstream of the h2 collimator.

\subsection{Controls Software}

The added complexity of three additional collimators per ring required a change to the controls software. During previous runs, manually inserting the collimators and reducing backgrounds took approximately 30 minutes after steering all of the interaction regions. RHIC experiments could not take data during this time and lost valuable integrated luminosity. It was decided that the new controls software should insert the collimators automatically to reduce the time necessary to bring the collimators into position.

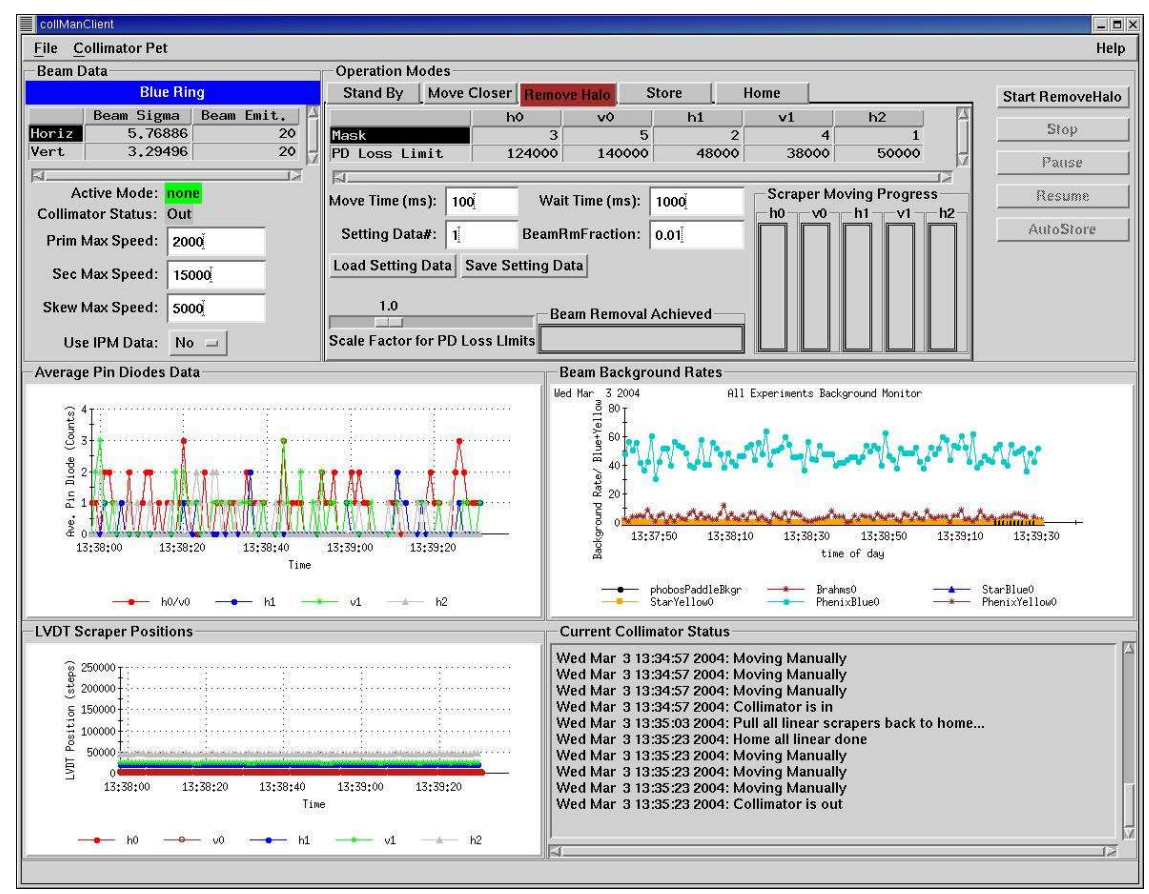

Figure 7.7: The Collimator Control Program for the Upgraded Collimation System

The collMan program written by Wenge Fu and Angelika Drees achieves these goals [92]. Figure 7.7 shows a screen shot of the associated application. This program replaces the scraper control program discussed in Section 3.3.3. The display shows 
the ring, rms beam size at primary scraper, mode of collimator operation, collimator status, and graphs of the average PIN diode rate at each scraper, the scraper positions, and experiment backgrounds.

The collimators operate in five modes:

- Stand By - moves the scrapers in parallel to a standby location not touching the beam.

- Move Closer - moves scrapers sequentially until the PIN diode rates reach a maximum value. This mode is used to touch halo.

- Remove Halo - moves scrapers sequentially until the PIN diode rates reach a more aggressive maximum value. This mode was not used during operations.

- Store - moves scrapers in parallel, using the design $\beta$ functions, to reduce PHENIX backgrounds.

- Home - retracts the collimators in parallel to the fully retracted position.

Each of these modes is used to move the scrapers progressively closer to the beam. The Move Closer and Remove Halo modes are computer controlled, using the PIN diode rates for feedback. The point at which the scrapers stop is set by a maximum PIN diode rate loaded from a database. The order in which the scrapers move is set by a mask number when moving the scrapers closer to the beam. The store mode has two modes, manual and automatic. Both modes are used to optimize the background rates at all experiments while the automatic mode uses the PHENIX background as feedback. Each mode has a stop button to halt the motion of the scrapers. Pause and resume buttons are used to temporarily stop and restart the process of moving the scrapers. 


\subsection{Collimator Performance}

The upgraded collimation system was used for background reduction and gap cleaning during the FY2004 run. As with the previous runs, the PHOBOS detector did not benefit from collimation. The BRAHMS detector also did not benefit. STAR provided "blue halo" and "yellow halo" signals as discussed in Section 6.3. PHENIX provided scintillator signals similar to those discussed in Section 6.3. These signals were the focus of background reduction efforts during the run.

Figure 7.8 shows the effect of the blue collimators on the STAR and PHENIX backgrounds. The effect of each collimator is easily seen. The collimators move sequentially in this figure.

Figure 7.9 shows the STAR and PHENIX background when the collimators are

\section{Experiment Backgrounds}

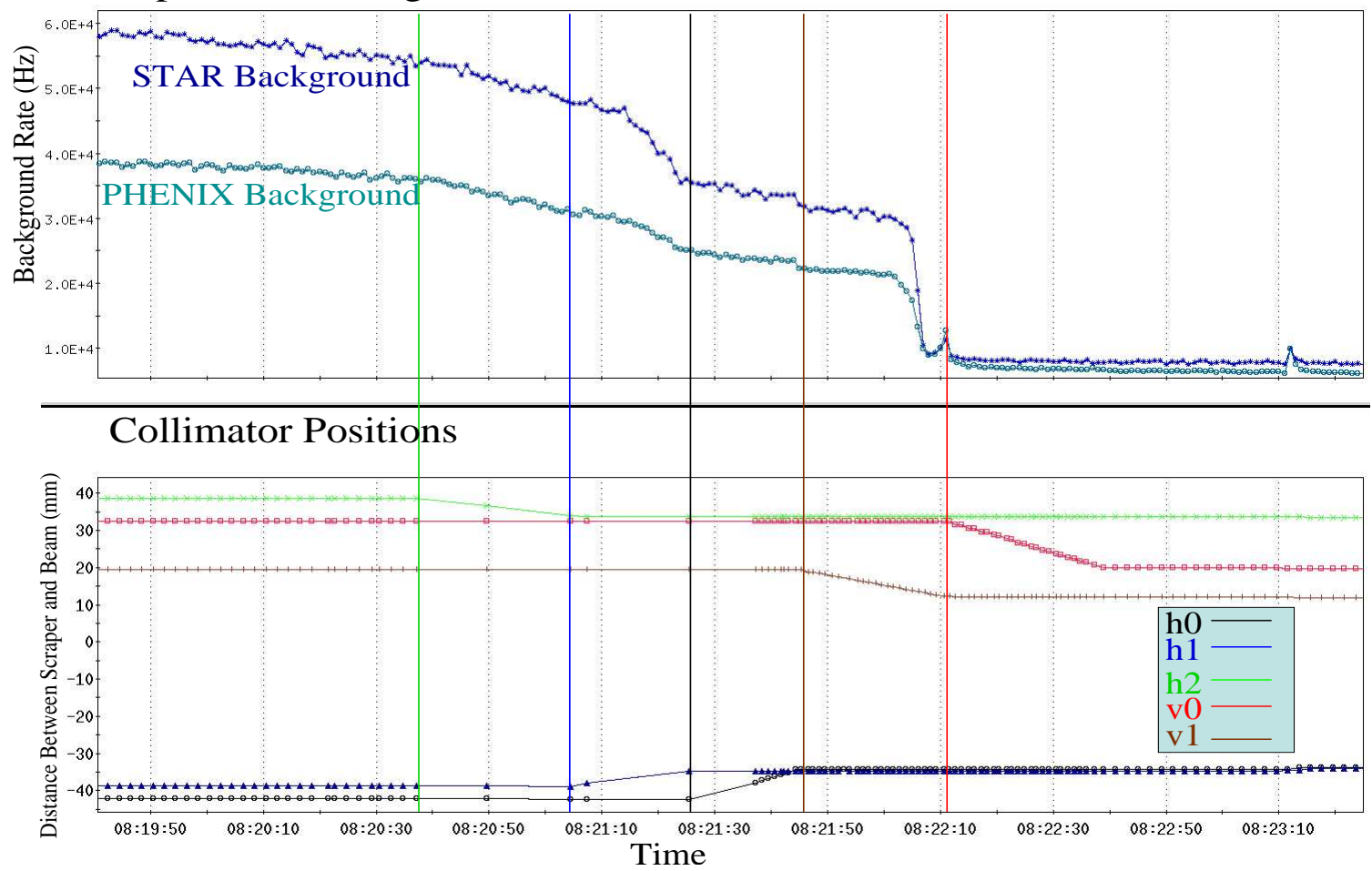

Figure 7.8: Blue Beam Background for STAR and PHENIX and Scraper positions for Fill 04854. The vertical lines denote when each collimator moves. 


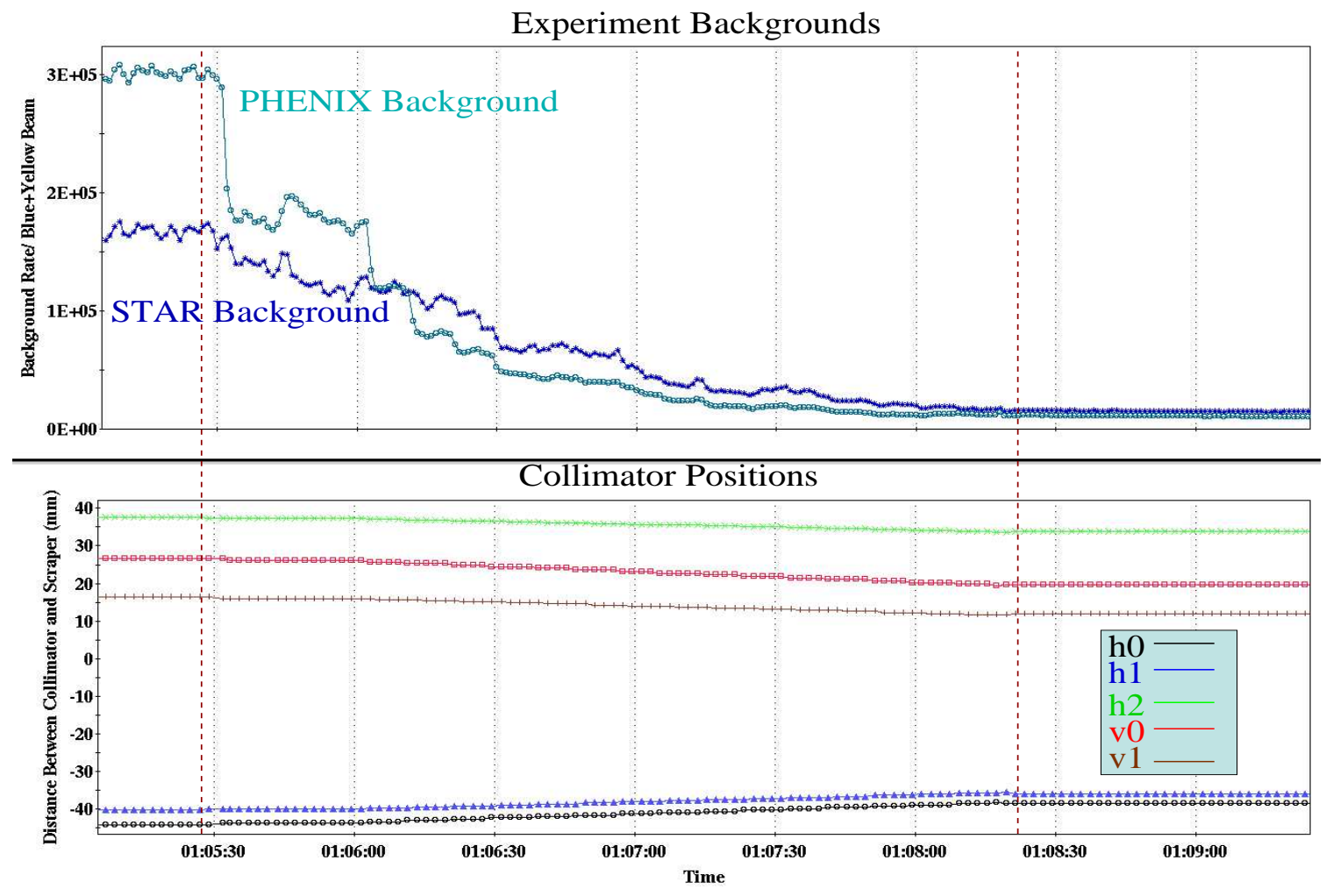

Figure 7.9: Blue Beam Background for STAR and PHENIX and scraper positions for Fill 04436. The vertical lines denote when the collimators start and stop moving simultaneously.

moved in parallel. The collimators in each plane were adjusted to be at the same betatron amplitude prior to the this. At the first vertical red line the collimators move together to reduce the experiment backgrounds while maintaining the same relative position among the collimators in each plane. The collimators stop moving at the second vertical red line.

Table 7.2 shows the average ratio of uncollimated to collimated background for the STAR and PHENIX detectors over six stores during the end of the FY2004 gold-gold run and the FY2003 deuteron - gold run (taken from Table 6.4). The upgraded collimation system was successful in further reducing the background from the both beams as compared to the single scraper used in the previous run. The background was reduced at least a factor of two more than the previous run with the 
Table 7.2: Background reduction in FY2004 run

\begin{tabular}{|c|c|c||c|}
\hline & \multicolumn{3}{|c|}{$\begin{array}{c}\text { Ratio of Uncollimated to } \\
\text { Collimated Background }\end{array}$} \\
\hline Detector & $\mathrm{d}-\mathrm{Au}-\beta^{*}=4 \mathrm{~m}$ & $\mathrm{~d}-\mathrm{Au}-\beta^{*}=3 \mathrm{~m}$ & $\mathbf{F Y 2 0 0 4} \beta^{*}=\mathbf{3} \mathrm{m}$ \\
\hline STAR Blue & 1 & 5 & $\mathbf{1 1}$ \\
STAR Yellow & 2 & 1 & $\mathbf{3}$ \\
PHENIX Blue & 2 & 4 & $\mathbf{1 1}$ \\
PHENIX Yellow & 4 & 1 & $\mathbf{4}$ \\
\hline$\beta^{*}$ is the $\beta$ function at the PHOBOS detector. $\beta_{S T A R}^{*}=\beta_{P H E N I X}^{*}=2 \mathrm{~m}$ during the
\end{tabular}

$\mathrm{d}-\mathrm{Au}$ run. $\beta_{S T A R}^{*}=\beta_{P H E N I X}^{*}=1 \mathrm{~m}$ for FY2004.

same $\beta_{P H O B O S}^{*}=3 \mathrm{~m}$ lattice.

Figure 7.10 shows the loss pattern around the RHIC rings during Fill 04854. The losses are localized to the collimator region. At 11:42 a blue horizontal dipole corrector (bo11-th10) tripped, and was reset at 11:51. During this time, the blue

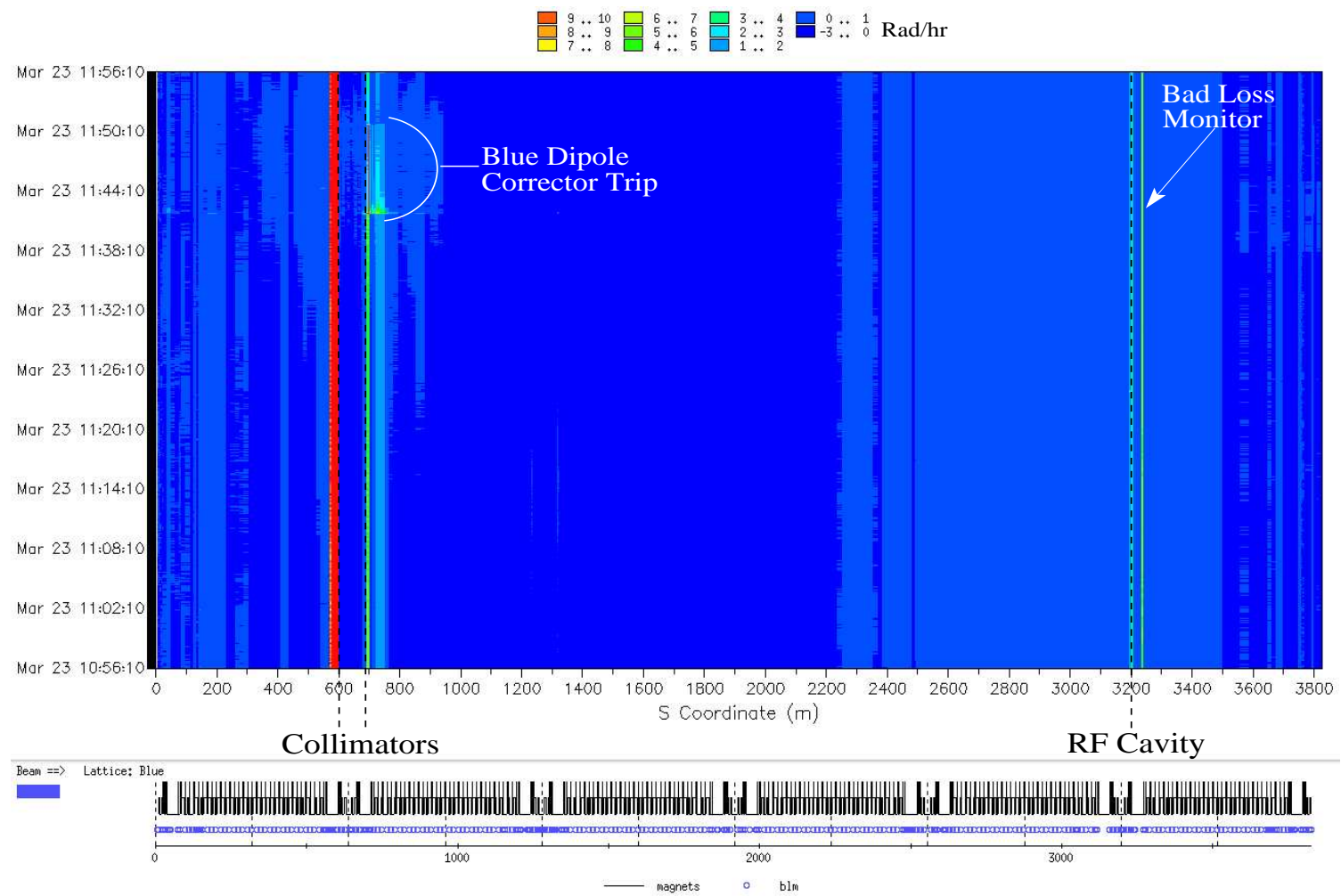

Figure 7.10: Loss Pattern around RHIC during Fill 04854. Losses are localized to the warm sections containing the collimators. 
collimators do not contain the losses. The scraping in the common RF cavity is due to the yellow beam, as discussed in Section 6.3. Figure 7.10 shows that the secondary collimators are effective in isolating the losses to the section that contains the collimators. This is to be compared with Figure 6.11 which shows losses distributed around the rings with only a single collimator.

Figure 7.11 shows a comparison of simulation and measured beam losses around the RHIC blue ring during Fill 04854. The losses at $s \approx 600 \mathrm{~m}$ are due to the yellow scraper. The losses at the RF cavity are due to the yellow beam. The other losses in the data with $s>2200 \mathrm{~m}$ are not real. The pedestals for these BLMs are not optimally set. The simulation in the figure includes warm and cold space apertures and uses protons.

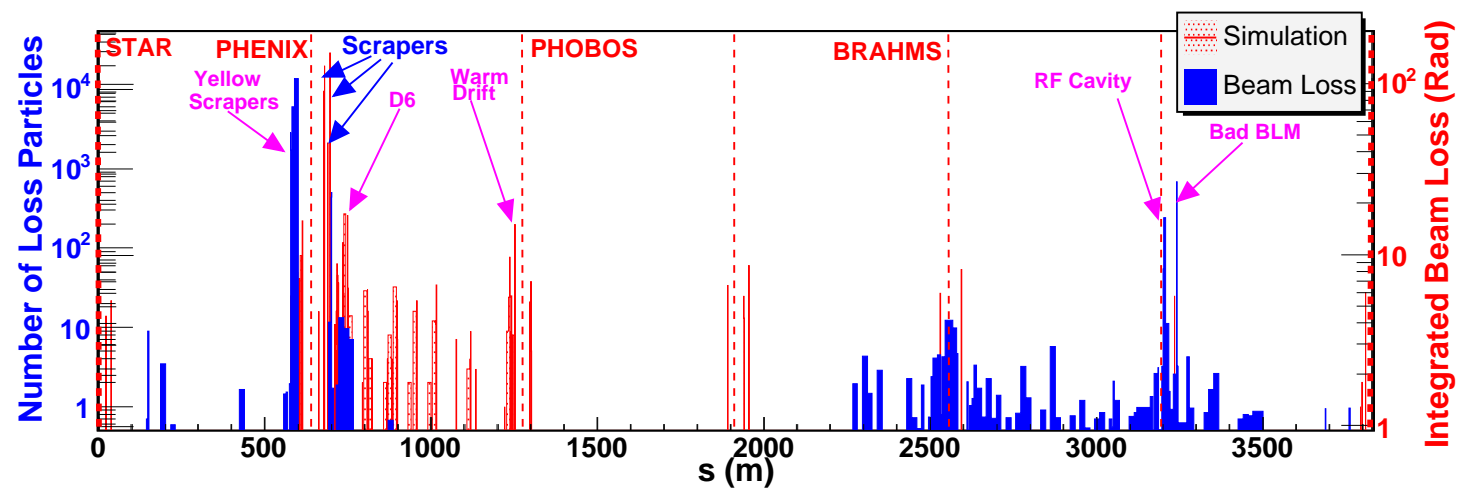

Figure 7.11: Comparison of the loss locations in RHIC to simulations of the blue ring for Fill 04854. The loss located at RF cavity is from the yellow ring. Integration time of the data is $100 \mathrm{~s}$. Vertical axes are log scale. The simulation has all horizontal collimators set at $6 \sigma_{x}$.

The data shows that the losses do not propagate down the 8 o'clock arc. The simulations show that the losses move further down the arc. These simulated losses are an artifact of the simulation. The horizontal scrapers are set at $6 \sigma_{x}$. The chosen particle distribution has particles at positions larger than $6 \sigma_{x}$. Particles that do not strike the primary scraper on the first pass, can strike the h2 secondary collimator. 
For these particles, h2 is a primary collimator and the scattered particles from this scraper will propagate into the arc. Simulated losses on either side of the interaction regions are from scraping in the warm space prior to the triplet magnet or the triplet itself. These are expected. Both the data and simulations show that the losses in the D6 region are one tenth of those in the scraper region.

We can use the same definition of the scattering reduction as in Section 6.3 and include all of the scrapers.

$$
\epsilon=\frac{\text { total lost particles }}{\text { total lost particles }- \text { particles lost at scrapers }}
$$

The simulation in Figure 7.11 has a scattering reduction of $\epsilon=5.1 \pm 0.1$. Simulations not using the secondary collimators, but otherwise identical, have a scattering reduction of $\epsilon=3.4 \pm 0.1$. These simulations have a lower background reduction than the simulations of Section 6.3. Those simulations did not include all machine apertures. The simulations in this chapter include the cold, as well as warm space apertures and therefore there are more locations for particles to be lost.

Simulations including the effect of the vertical collimators with more accurate particle distributions still need to be done.

\subsection{Future Directions}

Two additional vertical secondary collimators will be added for the FY2005 run, one in each ring. The goal of these additional collimators is to intercept the particles that are not intercepted with the first vertical secondary collimator. The placement is still limited by the available warm space in RHIC.

The yellow vertical secondary will be added upstream of the STAR detector with a phase advance $\Delta \phi \approx 315^{\circ}$ from the vertical primary. Placing the collimator 
downstream of STAR is not an option because the phase advance $\Delta \phi \approx 285^{\circ}$. It is also the injection area and space is restricted there. The collimator is in a location where the tunnel is not surrounded with a waterproof liner. Monitoring during the next run will determine if a liner needs to be installed at this location later.

The blue vertical secondary will be placed downstream of the PHOBOS detector with a phase advance of $\Delta \phi \approx 135^{\circ}$. This is the location of the blue beam dump. The abort kicker kicks in the horizontal plane, so striking the collimator with the entire beam is not an issue. Since the beam dump is in this section, the tunnel is already surrounded by a liner and groundwater contamination is not an issue.

These additional collimators will bring the total number of collimators in each ring to five, with a shared primary and two secondary collimators in each plane. 


\section{Conclusion}

We have demonstrated bent crystal channeling in the RHIC yellow ring using a bent silicon crystal during the FY2001 through FY2003 runs.

We developed an analytical model and used Monte Carlo simulations to estimate the channeling efficiency. These tools allow us to compute the change in crystal alignment as a function of the distance between the crystal and the beam core, the divergence of the beam that strikes the crystal, and channeling efficiency of the crystal.

Measurements of $\sigma_{x x^{\prime}} / \sigma_{x}^{2}$ show an increase of $66 \%$ compared to our model. This is traced back to errors in the Twiss parameters at the crystal collimator. Independent measurements of the Twiss parameters agree with our measurements in most cases.

The divergence of the beam that strikes the crystal is approximately 3-4 times larger than predicted by our model. Errors in the Twiss parameters cannot account for this discrepancy alone. Our model assumes that the beam has a gaussian distribution. Profile measurements with the scrapers show non-gaussian tails. A different ansatz of the particle distribution may be used to account for these effects, however this was never tried.

The predicted channeling efficiency is 4 times larger than the measured values. The predicted efficiency relies on the predicted beam divergence hitting the crystal. 
If the measured beam divergence is used to the predict channeling efficiency, the agreement is better than $20 \%$ in most cases.

We conclude that accurate knowledge of the transverse phase space is necessary when predicting the performance of crystal channeling from this.

Multiturn Monte Carlo simulations using CATCH include particles that return to the crystal after scattering in crystal. The depth of the volume capture region in the channeling data is dependent on the number of turns in the simulation. An estimate of the number of turns that particles survive after scattering in the crystal can be obtained using these simulations. The survival of the particles was shown to depend on the $\beta_{P H E N I X}^{*}$, and was 20 to 80 turns in all cases.

Collimation using the bent crystal was unsuccessful because of the low channeling efficiency. More particles were scattered from the crystal than were channeled. One secondary absorber was not enough to intercept all of the particles coming from the crystal. Using more than one secondary absorber defeats the purpose of using a bent crystal for the primary collimator. Background at the STAR detector downstream of the crystal collimator was increased by as much as a factor of two and rarely was reduced below the uncollimated background rate.

We used copper scrapers during operation for background reduction in some of the RHIC runs, reducing the backgrounds in the various experiments by as much as a factor of 10 . We used the scraper along with the tunemeter kicker to remove beam that accumulated in the abort gap. We measured beam profiles with the scrapers. The emittance from the collimator measurements agree to within $30 \%$ of measurements using the IPM if approximately $80 \%$ or more of the beam is removed. Diffusion measurements show a large scatter in the magnitude of the diffusion coefficient which is not understood.

Because crystal collimation was unsuccessful, the crystal was removed from 
RHIC and the collimation system was upgraded for the FY2004 RHIC run. Three secondary collimators were installed in the same warm section as the primary copper scraper. The upgraded collimation system was able to reduce backgrounds in the STAR and PHENIX detector by as much as 3 times more than the single scraper. Additional collimators will be installed for the FY2005 run. 


\section{Bibliography}

[1] J. Stark, Phys. Z. 13, 973 (1912).

[2] M. T. Robinson and O. S. Oen, Phys. Rev., 132 (6), 2385 (1963).

[3] P. Rol, et. al. in Proc. Fouth Inter. Conf. on Ionization Phenomena in Gases, edited by N. R. Nillson (North-Holland, Amsterdam, 1960), p.257; J. A. Davies et. al., Can. J. Chem. 28, 1526 (1960); 28, 1535 (1960).

[4] J. Lindhard. K. Dan. Viddensk Selsk. Mat. Phys. Medd. 34 (1) (1965).

[5] E. N. Tsyganov, Fermilab Internal Reports TM-682, TM-684 (1976).

[6] A. F. Elishev et. al., Phys. Lett. 88B, 387 (1979).

[7] J. Bak et. al., Phys. Lett. 93B, 505 (1980).

[8] W. M. Gibson et. al., Nucl. Instrum. Methods B 2, 54 (1984).

[9] V. M. Biryukov, Y. A. Chesnokov, and V. I. Kotov, Crystal Channeling and Its Application at High Energy Accelerators. (Springer-Verlag, Berlin, Heidelburg, 1997).

[10] R. A. Carrigan Jr. et. al., Phys. Rev. ST AB 5, 043501 (2002); Jukka Klem, Proton Extraction from a High Energy Beam with Bent Crystals Helsinki Institute of Physics Internal Report 1998-02 (1998); Relativistic Channeling and Related Coherent Phenomena 2004. http://www.lnf.infn.it/conference/rc2004/

[11] D. Trbojevic et. al., A study of RHIC Crystal Collimation. Proceedings of the 1998 European Particle Accelerator Conference, Stockholm, Sweden.

[12] V. Radeka, Heavy Ion Microbeam and Micron Resolution Detector. NSBRI Grant Proposal No. NSBRI00-01. 
[13] http://www.agsrhichome.bnl.gov/NT-share/rhicdm/00_toc1i.htm

[14] http://www.bnl.gov/RHIC/STAR.htm

[15] http://www.phenix.bnl.gov/phenix/WWW/intro/index.html

[16] http://www.phobos.bnl.gov/overview.htm

[17] M. Adamczyk, et. al., Nucl. Instrum. Methods A 499, 437 (2003).

[18] http://www.rhic.bnl.gov/pp2pp/

[19] D. A. Edwards and M. J. Syphers, An Introduction of the Physics of High Energy Accelerators (John Wiley \& Sons, Inc., New York, 1993).

[20] S. Y. Lee, Accelerator Physics (World Scientific Publishing Co. Pte. Ltd., Singapore, 1999).

[21] J. Uythoven, et. al., LHC Beam Dumping System: Failure Senarios and Reliability Aspects. Proceedings of the at 2003 Particle Accelerator Conference, Portland, Oregon.

[22] W. Fischer, et. al., Intra-Beam Scattering Measurements in RHIC. Proceedings of the at 2002 European Particle Accelerator Conference, Paris, France.

[23] A. Piwinski, Intra-Beam Scattering. Proceedings of the $9^{\text {th }}$ Int. Conf. on High Energy Acc., Stanford. (1974) p.405.

[24] J. D. Bjorken and S. K. Mtingwa, Particle Accelerators 13, 115 (1983).

[25] A. Piwinski, Touschek Effect and Intrabeam Scattering in Handbook of Accelerator Physics and Engineering edited by Alexander Wu Chao and Maury Tigner. (World Scientific Publishing Co. Pte. Ltd., 1999).

[26] W. Fischer, et. al., Measurements of Intra-Beam Scattering Growth Times with Gold Beam below Transition in RHIC. Proceedings of the at 2001 Particle Accelerator Conference, Chicago, Illinois; W. Fischer, et. al., Beam Lifetime and Emittance Growth Measurements of Gold Beams in RHIC at Storage. ibid.

[27] K. Hirata, Beam-Beam Effects in Storage Rings in Handbook of Accelerator Physics and Engineering edited by Alexander Wu Chao and Maury Tigner. (World Scientific Publishing Co. Pte. Ltd., 1999).

[28] W. Fischer and S. G. Peggs, RHIC as a Test Bench For Beam-Beam Studies. C-A/AP Note 61, Brookhaven National Laboratory (2001). 
[29] W. Fischer, et. al., Vacuum Pressure Rise with Intense Ion Beams in RHIC. Proceedings of the at 2002 European Particle Accelerator Conference, Paris, France; H. C. Hseuh, et. al., Analysis of Beam Induced Pressure Increases in RHIC Warm Vacuum Sections. ibid.

[30] W. Fischer et. al., Electron Clouds and vacuum pressure rise in RHIC. Proceedings of ECLOUD'04, Napa Valley, April 2004; U. Iriso et. al., Analysis of Electron Cloud at RHIC. Proceedings of the 2004 European Particle Accelerator Conference, Lucern, Switzerland.

[31] F. Zimmermann, Transverse Proton Diffusion. Technical Report SLACPUB-6634 Stanford Linear Accelerator Center, 1994.

[32] M. Syphers, Emittance Dilution Effects in Handbook of Accelerator Physics and Engineering edited by Alexander Wu Chao and Maury Tigner. (World Scientific Publishing Co. Pte. Ltd., 1999).

[33] M.Seidel, The Proton Collimation System of HERA. DESY Report 94103, Universität Hamburg, June 1994.

[34] M. N. Rudden and J. Wilson, Elements of Solid State Physics (John Wiley \& Sons Ltd., West Sussex, second edition, 1993); C. Kittel. Introduction to Solid State Physics (John Wiley \& Sons Inc., New York, seventh edition, 1996).

[35] http://nina.ecse.rpi.edu/shur/SDM1/Notes/06Bonds.pdf

[36] http://www.ee.byu.edu/cleanroom/EW_orientation.phtml

[37] A. A. Elishev, et. al., Phys. Lett. B 88, 387 (1979).

[38] James A. Ellison, Theory of particle motion in straight and distorted crystals in Relativistic Channeling edited by Richard A. Carrigan Jr. and James A. Ellison (NATO ASI Series B Vol. 165, Plenum Press, New York, 1987).

[39] G. K. Woodgate, Elementary Atomic Structure (Oxford University Press, Oxford, U.K., second edition, 1998).

[40] K. Hagiwara et. al., Phys. Rev. D 66, 010001 (2002).

[41] L. D. Landau, J. Exp. Phys. (USSR) 8, 201 (1994); D. H. Wilkinson, Nucl. Instrum. Methods A 383, 513 (1996).

[42] H. Esbensen and J. A. Golovchenko, Nucl. Phys. A 298, 382 (1978); H. Esbensen, et. al., Phys. Rev. B 8, 1039 (1978). 
[43] S. P. Møller, Energy Loss and Straggling of Random and Channeled HighEnergy Particles in Thin Targets in Relativistic Channeling edited by Richard A. Carrigan Jr. and James A. Ellison (NATO ASI Series B Vol. 165, Plenum Press, New York, 1987).

[44] Yoshi-Hiko Ohtsuki, Charged Beam Interaction with Solids (Taylor and Francis Inc., New York, 1983).

[45] Y. H. Ohtsuki and H. Nitta, Theory of Dechanneling in Relativistic Channeling edited by Richard A. Carrigan Jr. and James A. Ellison (NATO ASI Series B Vol. 165, Plenum Press, New York, 1987).

[46] V. Biryukov, Phys. Lett. A 205, 340 (1995).

[47] R. Aßman, The LHC collimation project - implementation of a phased approach. External Review of LHC Collimation Project. June 30, 2004 http://lhc-collimation-project.web.cern.ch/lhc-collimation-project /review04-presentations/assmann-project-overview-e.pdf

[48] Nuria Catalán Lasheras, Transverse and Longitudinal Beam Collimation in a High Energy Collider (LHC). Ph.D. Thesis, Universidad de Zaragoza, Nov 1998.

[49] T. Trenkler and J.B. Jeanneret, The principles of two stage betatron and momentum collimation in circular accelerators. CERN SL Note 95-03, LHC 312, 1995.

[50] W. Feller, An Introduction to Probability Theory and Its Applications. Volume II (John Wiley \& Sons, Inc., second edition, 1971).

[51] P. Thompson et. al., in Beam Instrumentation Workshop 2000, edited by Kenneth D. Jacobs and R. Coles Sibley III (American Institute of Physics, 2000).

[52] J. Bosser and G. Ferioli, Measurements of the Relative Sensitivities Between Some Beam-Loss Monitors: Part I. CERN SL/BI Note 97-01, CERN PS/BD Note 97-01, 1997.

[53] R. Fliller III and A. Drees, Beam Profile Measurements and PIN Diode Calibration Using the RHIC Collimators, C-A/AP Note 132 Brookhaven National Laboratory (2004).

[54] http://www.agsrhichome.bnl.gov/Controls/doc/pet/pet.html

[55] L. R. Hammons III, MSI Minor Project Final Presentation. Stony Brook University, 2001.

[56] http://www.agsrhichome.bnl.gov/Controls/doc/Collimator/Collimator.html 
[57] http://www.agsrhichome.bnl.gov/Controls/doc/Logger/Logger.html

[58] http://www.agsrhichome.bnl.gov/Controls/doc/Gpm/

[59] http://www.aps.anl.gov/asd/oag/manuals/SDDStoolkit/node108.html

[60] Rene Brun and Fons Rademakers, ROOT - An Object Oriented Data Analysis Framework, Proceedings AIHENP'96 Workshop, Lausanne, Sep. 1996, Nucl. Instrum. Methods in Phys. Res. A 389 (1997) 81-86. See also http://root.cern.ch/

[61] V. Biryukov, CATCH 1.4 User's Guide. CERN SL Note 93-74 (AP), 1993. See also [9].

[62] T. Trenkler and J. B. Jeanneret, K2: A software package evaluating collimation systems in circular colliders. CERN SL Note 94-105 (AP), 1994.

[63] F. W. Jones, G. H. Mackenzie and H. Schönauer, ACCSIM - A program to simulate the accumulation of intense proton beams. Proc. 14th International Conference on High Energy Accelerators, in Particle Accelerators 31, 199 (1990). http://www.triumf.ca/compserv/accsim.html

[64] N. Malitsky and R. Talman, Unified Accelerator Libraries, AIP 391, Williamsburg, 1996; N. Malitsky and R. Talman, Status of Unified Accelerator Libraries, IEEE Particle Accelerator Conference, p. 2434, 1997.

[65] H. Gould and J. Tobochnik, Computer Simulation in Physics Part I (AddisonWesley, Reading, MA 1988).

[66] L. Schachinger and R. Talman, Particle Accelerators 22, 35 (1987).

[67] J. Wei, Longitudinal Dynamics of the Non-Adiabatic Regime on AlternatingGradient Synchrotrons. Ph.D. Thesis, Stony Brook (1990).

[68] http://www.ual.bnl.gov

[69] M. Frigo and S. G. Johnson, FFTW: An Adaptive Software Architecture for the FFT Proceedings of the 1998 International Conference on Acoustics, Speech and Signal Processing vol. 3, pp. 1381-1384.

[70] Radoslav Adzic, Private Communication.

[71] Philip R. Bevington and D. Keith Robinson, Data Reduction and Error Analysis for the Physical Sciences. (McGraw-Hill,Inc., New York, second edition, 1992). 
[72] T.Satogata, et. al., Linear Optics During the RHIC 2001-2 Run. Proceeding of the 2002 European Particle Accelerator Conference, Paris, France; T.Satogata, et. al., Linear Optics Measurement and Correction in the RHIC 2003 Run.Proceedings of the 2003 Particle Accelerator Conference, Portland, Oregon; M. Bai, et. al., Measurement of Betatron Functions and Phase Advances in RHIC with AC Dipoles. ibid.

[73] Michael Blaskiewicz, Private Communication.

[74] Bergoz Beam Instrumentation. http://www.bergoz.com/

[75] T. Satogata, Private Communication.

[76] A. Drees, Private Communication.

[77] A. Drees, R. P. Filler III, et. al., RHIC Collimator Performance. Proceedings of the 2002 European Particle Accelerator Conference, Paris, France.

[78] R. P. Filler III, A. Drees, RHIC Collimation. Proceedings of the RHIC Retreat 2003.; http://www.agsrhichome.bnl.gov/AP/RHIC2003/Retreat/

03_Wed_AM/08_Fliller_Collimation.ppt

[79] A. Drees and Z. Xu, Results From Luminosity Scans During the RHIC 2000 Run. Proceedings of the 2001 Particle Accelerator Conference, Chicago, Illinois.

[80] Vasily Dzhordzhadze, MuID for the PHENIX Experiment. http://www.phenix/bnl.gov/WWW/run/03/focus/talks/mui/MuIDFocus.html

[81] S. Peggs and G.F. Dell, Momentum collimation at Q9. RHIC/AP Note 78, Brookhaven National Laboratory (1995); D. Trbojevic, A. Stevens, M. Harrison, F. Dell, S. Peggs, A Study of Betatron and Momentum Collimators in RHIC RHIC/AP Note 142, Brookhaven National Laboratory (1997); D. Trbojevic, A. Stevens, M. Harrison, A Study of Betatron and Momentum Collimators in RHIC. RHIC/AP Note 143, Brookhaven National Laboratory (1997).

[82] A. Drees, et. al., Abort Gap Studies and Cleaning during RHIC Heavy Ion Operation. Proceedings of the 2003 Particle Accelerator Conference, Portland, Oregon; A. Drees, et. al., Abort Gap Cleaning in RHIC. Proceedings of the at 2002 European Particle Accelerator Conference, Paris, France.

[83] A. Drees, R. Michnoff, M. Brennan, J. DeLong in Beam Instrumentation Workshop 2000, edited by Kenneth D. Jacobs and R. Coles Sibley III (American Institute of Physics, 2000).

[84] http://www.agsrhichome.bnl.gov/Controls/doc/GapCleaner/GapCleaner.html 
[85] P. R. Cameron et. al., The RHIC Wall Current Monitor System, Proceedings of the 1999 Particle Accelerator Conference, New York City, New York.

[86] A. Drees, et. al., Continuous Abort Gap Cleaning at RHIC. Proceedings of the 2004 European Particle Accelerator Conference, Lucern, Switzerland.

[87] R. P. Fliller III, et. al., Beam Diffusion Measurements at RHIC. Proceedings of the 2003 Particle Accelerator Conference, Portland, Oregon; R. P. Fliller III, et. al., Beam Diffusion Studies at RHIC. Proceedings of the 2002 European Particle Accelerator Conference, Paris, France.

[88] A. Drees, et. al., Results from Vernier Scans at RHIC During Runs 2000-2003. Proceedings of the 2003 Particle Accelerator Conference, Portland, Oregon.

[89] F. Pilat, et. al., Nonlinear Effects in the RHIC Interaction Regions: Modeling, Measurements, Correction. Proceedings of the 2003 Particle Accelerator Conference, Portland, Oregon.

[90] W. H. Press, S. A. Teukolsky, W. T. Vetterling, and B. P. Flannery, Numerical Recipes in C. 2nd Edition (Cambridge University Press, Cambridge, 1992).

[91] Peter Cameron, Private Communication.

[92] http://www.agsrhichome.bnl.gov/Controls/doc/CollMan/collMan.html; http://www.agsrhichome.bnl.gov/Controls/doc/collManClient/collManClient.html

[93] Dick Hseuh and Dan Weiss, Private Communication.

[94] Steve Tepikian, Private Communication.

[95] MADX User's Guide. http://frs.home.cern.ch/frs/Xdoc/uguide.html

[96] H. Grote, et. al., SXF (Standard eXchange Format): definition, syntax, examples. RHIC/AP Note 155, Brookhaven National Laboratory (1998). 


\section{Appendix A}

\section{Constructing the Aperture Model}

The original aperture model for RHIC contained only the apertures of the magnets. All warm spaces were assumed to have no aperture limits. In reality, the warm spaces are composed of $10 \mathrm{~cm}$ inner diameter (ID) vacuum pipe with restrictions from instrumentation, kickers, experimental beampipes, etc. The rms beam sizes can be as large as $5 \mathrm{~mm}$ in each plane in these sections.

We needed to include the warm spaces in the aperture model for more accurate modeling of beam losses and experimental backgrounds. This appendix discusses the steps involved to generate lattice files containing an accurate aperture description for simulations and loss patterns like those in Section 6.3.

We devised a semi-automated procedure to incorporate these apertures. This requires a number of steps:

1. generating a list of apertures.

2. inserting apertures into the lattice database.

3. incorporating these into the lattice file as markers.

\section{Generating the Aperture List}

Generating the aperture list is the most "hands-on" portion of the process. First, one must obtain the components list for the warm sections. This is obtainable from the RHIC vacuum group, as an Excel file containing a set of spreadsheets [93]. Each of the spreadsheets contains the component list for each warm section. Some spreadsheets are specific to a particular section, others contain information for multiple sections that are identical. A screen shot of the yellow ring 11 o'clock warm section spreadsheet is shown in Figure A.1.

The user must do the following:

1. Go through each spreadsheet and ensure that all of the $\mathbf{I D} / \mathbf{H x V}$ spaces are filled. The $\mathbf{I D} / \mathbf{H x V}$ lists the horizontal and vertical inner dimension of each 


\begin{tabular}{|c|c|c|c|c|c|c|c|c|c|}
\hline & A & B & C & D & E & $\mathrm{F}$ & G & $\mathrm{H}$ & 1 \\
\hline \multicolumn{10}{|l|}{1} \\
\hline 2 & & \multicolumn{8}{|c|}{ Layout of YI11 with Stochastic cooling pickup tank } \\
\hline 3 & & & & & & & & \multicolumn{2}{|l|}{$06 / 17 / 03$} \\
\hline 4 & & Component & Part No. & \multicolumn{2}{|c|}{ Distance to IP } & \multicolumn{2}{|c|}{ Part Length } & \begin{tabular}{|l|l} 
flangeOD \\
\end{tabular} & $\sim \mathrm{ID} / \mathrm{HxV}$ \\
\hline 5 & & & & $(\mathrm{~m})$ & (in) & (in) & $(\mathrm{cm})$ & \begin{tabular}{|l|l} 
(in) \\
\end{tabular} & $(\mathrm{cm})$ \\
\hline 6 & & Q3 W-C & & 38.439 & 1513.33 & 740.82 & 1881.69 & 6.75 & \\
\hline 7 & & Gate Valve & $43035007-2$ & 38.536 & 1517.17 & 3.84 & 9.75 & 6.75 & 12.5 \\
\hline 8 & & Bellows-s & $43035004-4$ & 38.731 & 1524.84 & 7.67 & 19.48 & 6.75 & 11.6 \\
\hline 9 & & Tee w/ Pump & 43015011 & 39.064 & 1537.96 & 13.12 & 33.32 & 6.75 & 12.1 \\
\hline 10 & & Bellows-I & $43035004-1$ & 39.357 & 1549.48 & 11.52 & 29.26 & 6.75 & 11.6 \\
\hline 11 & & Pipe & 43035019 & 44.553 & 1754.06 & 204.58 & 519.63 & 6.75 & 12.1 \\
\hline 12 & & Pipe & 43035019 & 49.749 & 1958.64 & 204.58 & 519.63 & 6.75 & 12.1 \\
\hline 13 & & Pipe & 43035019 & 54.946 & 2163.21 & 204.58 & 519.63 & 6.75 & 12.1 \\
\hline 14 & & Bellows-I & $43035004-1$ & 55.238 & 2174.73 & 11.52 & 29.26 & 6.75 & 11.6 \\
\hline 15 & & Tee w/ Pump & 43015011 & 55.571 & 2187.85 & 13.12 & 33.32 & 6.75 & 12.1 \\
\hline 16 & & Bellows-I & $43035004-1$ & 55.864 & 2199.37 & 11.52 & 29.26 & 6.75 & 11.6 \\
\hline 17 & & Pipe & 43035019 & 61.060 & 2403.95 & 204.58 & 519.63 & 6.75 & 12.1 \\
\hline 18 & & Pipe & 43035019 & 66.257 & 2608.53 & 204.58 & 519.63 & 6.75 & 12.1 \\
\hline 19 & & Pipe & 43035019 & 70.167 & 2762.50 & 153.97 & 391.08 & 6.75 & 12.1 \\
\hline 20 & & Transition & Pascal 5-3-CR & 70.396 & 2771.50 & 9.00 & 22.86 & $6.75-4.62$ & 7.34 \\
\hline 21 & & Bellows-welded & SBC-? & 70.570 & 2778.33 & 6.84 & 17.36 & 4.62 & 7.34 \\
\hline 22 & & Transition & FNAL-MC2918 & 70.655 & 2781.70 & 3.36 & 8.54 & $4.62-14$ & 7.34 \\
\hline
\end{tabular}

Figure A.1: Example spreadsheet of the components list for the Yellow 11 o'clock warm section.

component. Sometimes a particular component is listed on multiple spreadsheets and the aperture information is only recorded once.

2. Save each spreadsheet as a CSV file with the name $C S$.csv, where $C$ is $\mathrm{B}, \mathrm{Y}$, or IP for Blue, Yellow, or Interaction Point and $S$ is the arc or interaction region number (i.e.Y11.csv). Each sector and IP needs a separate file, so a shared spreadsheet must be saved under different names. There are 30 files total (12 blue, 12 yellow, and 6 IP files).

3. Use the PERL script apsforsteve.pl to generate a text file with the aperture list. At the command line type apsforsteve.pl ring outputfilename where outputfilename is BlueAperture.txt or YellowAperture.txt, and ring is Blue or Yellow. The colorAperture.txt files are tab delimited and contain the aperture names, $\mathrm{s}$ location, shape, horizontal size, and vertical size. The aperture naming scheme is ap $S \mathrm{P} N C$, where $S$ is the arc number, $N$ is the aperture number in that sector $(1,2$, etc.), and $C$ is either $\mathrm{B}, \mathrm{Y}$, or $\mathrm{G}$ for Blue, Yellow, or Green for interaction regions.

4. Give the spreadsheet and the colorAperture.txt files to the person in charge of generating the lattice files [94]. They will use these files to generate the lattice files. The user will use colorAperture.txt files in the next step.

All of the necessary files have now been generated to place the apertures into the database. 


\section{Inserting apertures into the database}

The PERL script apers2database.pl inserts the apertures into the database. The user enters apers2database.pl ring username password where ring is Blue or Yellow, username and password are the user's username and password for the database. The user needs insert and delete permissions in the rhic database aperture table. This file deletes all of the apertures named in BlueAperture.txt or YellowAperture.txt (depending on ring) from the table, and inserts the new apertures from the file to the database.

\section{Generating the lattice file}

The aperture locations are added into the MAD lattice files [95] for each ring by the person in change of the lattice files [94]. These apertures are entered as marker elements at the appropriate locations without the aperture shape and size defined. Fulvia Pilat uses the MAD lattice file to generate an SXF file [96] for use with UAL. The aperture remains undefined so that UAL and MAD users can simulate the lattice without the apertures for other studies.

The PERL script aper-filter.pl inserts the apertures into the SXF file. Typing aper-filter.pl inputfile outfile uses the SXF inputfile and the database to generate the SXF outfile with the aperture shapes and sizes defined. This defines all of the apertures, both the warm apertures and the magnet apertures. Using the outfile as input to UAL, one can perform simulations where particles can strike realistic apertures.

This entire procedure needs to be repeated every time the aperture configuration of RHIC changes. This needs particular attention after a shutdown when maintenance is done. 